\title{
InP Based Type-II Quantum Wells PIN Photodiodes
}

\author{
Dissertation \\ Presented to \\ the faculty of the School of Engineering and Applied Science \\ University of Virginia \\ In Partial Fulfillment \\ of the Requirements for the Degree \\ Doctor of Philosophy \\ Electrical Engineering \\ by
}

Baile Chen

May 2013 
C Copyright by

\section{Baile Chen}

All rights reserved

May 2013 


\section{APPROVAL SHEET}

The dissertation

is submitted in partial fulfillment of the requirements

for the degree of

Doctor of Philosophy

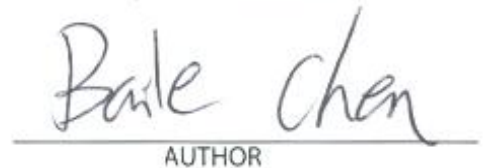

The dissertation has been read and approved by the examining committee:
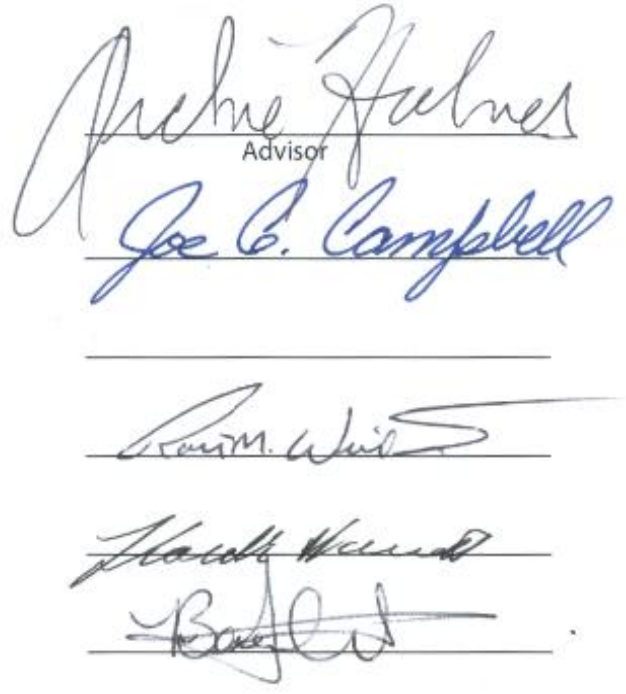

Accepted for the School of Engineering and Applied Science:

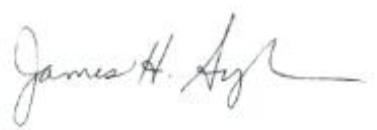

Dean, School of Engineering and Applied Science

May

2013 
This dissertation is dedicated to my parents and sisters for all the love, kindness, courage and support 


\section{Abstract}

Short wavelength infrared (SWIR) and mid wavelength infrared (MWIR) photodetectors have applications in areas such as chemical sensing, gas monitoring, medical diagnostics, infrared imaging and free-space communications. Mercury Cadmium Telluride (HgCdTe) is the predominant material system used in SWIR, MWIR and long wavelength infrared (LWIR) applications. However, HgCdTe often suffers from poor material uniformity and low yield. Comparable performance can be achieved on GaSb substrates with high quality InAs/GaSb strained-layer superlattices, but significant cooling is required to achieve high detectivity performance.

Indium Phosphide (InP) based InGaAs/GaAsSb type-II quantum wells photodiodes are the promising candidates for the detection in SWIR and MWIR region, especially at room temperature. These photodiodes can take advantage of mature material and device technology of InP material system. In this dissertation, I study these InP based type-II quantum wells photodiodes theoretically and experimentally. Six-band k·p modeling was used to design these type-II quantum well structures. It is found that in order to maximize the transition wavelength and wave function overlap under strain compensated condition, the thickness of the InGaAs layer should be larger than that of the GaAsSb layer, and the GaAsSb layer should be compressively strained, while InGaAs layer should be tensile strained. Both lattice matched and conventional strain compensated type-II quantum wells photodiodes are designed and studied experimentally in this dissertation. How the performance of these type-II photodiodes changes as the detection wavelength increases is studied by comparing the performance of both photodiodes. The research has shown that the device with 100 pairs of $7 \mathrm{~nm} \mathrm{In}_{0.34} \mathrm{Ga}_{0.66} \mathrm{As} / 5 \mathrm{~nm} \mathrm{GaAs}_{0.25} \mathrm{Sb}_{0.75}$ strain 
compensated type-II quantum wells absorption region has an optical response out to $3.2 \mu \mathrm{m}$, while the device with 100 pairs of $7 \mathrm{~nm} \mathrm{In}_{0.53} \mathrm{Ga}_{0.47} \mathrm{As} / 5 \mathrm{~nm} \mathrm{GaAs} \mathrm{ns}_{0.5} \mathrm{Sb}_{0.5}$ lattice matched type-II QWs absorption region has an optical response out to $2.7 \mu \mathrm{m}$. The strain compensated devices show detectivities of $1.4 \times 10^{9} \mathrm{~cm} \cdot \mathrm{Hz}^{1 / 2} \cdot \mathrm{W}^{-1}$ at $\lambda=2.7 \mu \mathrm{m}$ at $290 \mathrm{~K}$ and $1.5 \times 10^{10} \mathrm{~cm} \cdot \mathrm{Hz}^{1 / 2} \cdot \mathrm{W}^{-1}$ at $200 \mathrm{~K}$. For $\lambda=3.0 \mu \mathrm{m}$, the detectivity $\mathrm{D}^{*}$ is $2.0 \times 10^{8} \mathrm{~cm} \cdot \mathrm{Hz}^{1 / 2} \cdot \mathrm{W}^{-1}$ at $290 \mathrm{~K}$ and increases to $1.0 \times 10^{9} \mathrm{~cm} \cdot \mathrm{Hz}^{1 / 2} \cdot \mathrm{W}^{-1}$ at $200 \mathrm{~K}$. They are the first $3 \mu \mathrm{m}$ results demonstrated on InP substrate, using interband transition without lattice mismatch layers.

Moreover, in order to further extend the detection wavelength, the new strain compensated InGaAs/GaAsSb QWs PIN photodiode is studied, which can lead to much longer detection wavelengths for similar GaAsSb compositions based on the modeling. This new strain compensation concept is demonstrated experimentally to have a cut-off wavelength similar to that of conventional strain compensated sample but with lower $\mathrm{Sb}$ composition in the GaAsSb layer. In addition, the carrier transport mechanism in the type-II quantum wells is studied in order to improve the carrier collection efficiency, and it is found that the thermionic emission is the dominant way for the photo generated electrons to get out of the quantum wells. 


\section{Acknowledgement}

First of all, I would like to express my deepest appreciation and gratitude to my advisor, Dr. Archie L. Holmes Jr., for his invaluable guidance during my graduate study at University of Virginia. I have been amazingly fortunate to have such a nice and experienced advisor. In my opinion, Dr. Holmes is the best advisor that a student can have. His patience and support helped me overcome many difficulties during my research, and his insightful feedback always gave me confidence in my research. Sometimes, he was more than an academic advisor for me; he was also like my friend. He generously shared his experience as a friend with me to help me indentify my future career. I have benefitted immensely from Dr. Holmes, and he will forever have my deepest gratitude.

Second, I would also like to thank Dr. Joe C. Campbell who gave me many insightful comments and constructive criticism during my research and course work study. I have benefitted a lot from him. Furthermore, I really appreciate him for sharing all the lab equipments with my group, and serving as the chair of my dissertation committee. Furthermore, I am very grateful to Dr. Bora M. Onat of Princeton Lightwave Inc. for the helpful discussions during my research. My thanks also go to Dr. Robert M. Weikle II and Dr. Lloyd R. Harriott who also served on my dissertation committee. Their valuable feedbacks and discussions helped me to improve this dissertation in many ways. Moreover, I appreciate Dr. Avik Ghosh, who was also one of my proposal committees, for all the help of the simulation work during my Ph.D study.

I would like to thank my group members Dr. Weiyang Jiang and Jinrong Yuan. Dr. Weiyang Jiang has helped familiarize me with the material characterization and device 
fabrication process. The numerous and productive discussions with him greatly helped me in my initial understanding of my research area.

I would also like to thank all the members of the Dr. Campbell's research group at University of Virginia. In particular, I would like to acknowledge Chong Hu, Han-Din Liu, Dion C. McIntosh, Qiugui Zhou, Wenlu Sun, Andreas Beling, Yang Fu, Kejia Li, Lijun Li, Xiaoguang Zheng, Zhiwen Lu, Zhi Li, Huapu Pan, Allen S. Cross, Yaojia Chen, Hao Chen and Joseph Murray for many valuable discussions that helped me understand my research area better and led to many new research ideas.

I would like to thank Brenda E. Crider, Yadira Weaver, Dan R. Fetko, Eileen F. Thacker and Gloria R. Walker for their administrative help. In addition, I would like to thank Joe Beatrice, Harry H. Wade, Alex M. Lobo, David Durocher and Gary Li for all the technical support.

I would also like to thank my many friends at the University of Virginia. I really appreciate all the support and encouragement from them. I would like to especially acknowledge Zhiheng Xie, Keye Sun, Chunhu Zhang, Huilin Li, Yiran Xia, Guangfu Wang for their company whenever I felt helpless or lonely during my Ph.D career.

Last, but not least, I wish to express my gratitude to my family. My father Shengcong Chen, my mother Xiuyue Zhang, my sisters Jiandan Chen and Baiqiong Chen, and my niece Chenyao Ye have been supporting and loving me throughout the years. Without their support, I could never have achieved so much. 


\section{Table of Contents}

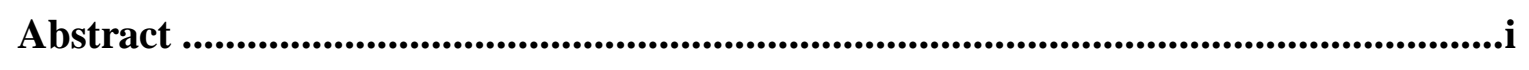

Acknowledgement ................................................................................................................... iii

Table of Figures......................................................................................................................viii

List of Tables ......................................................................................................................... xvi

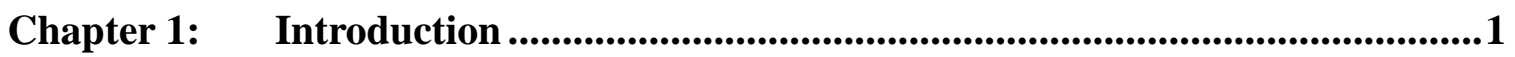

Chapter 2: $\quad$ Overview of Infrared Semiconductor Detectors.......................................6

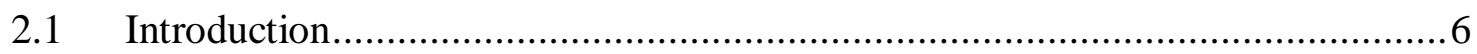

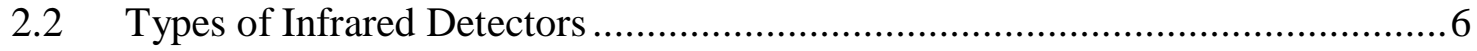

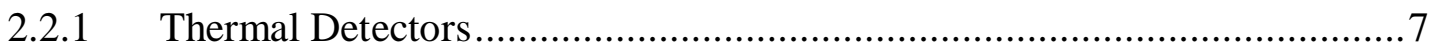

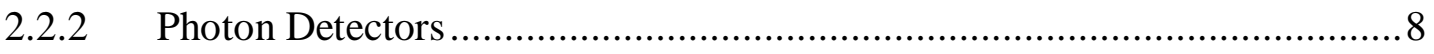

2.3 Material Systems for MWIR Photodetector ................................................... 8

2.3.1 IV-VI material systems ..................................................................

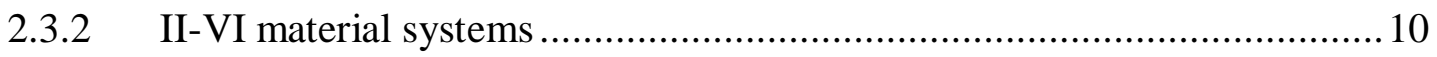

2.3.3 GaAs and InP Based QWIPs and QDIPs .............................................. 10

2.3.4 InSb and InAs Based Photodetectors ....................................................... 12

2.3.5 InAs/GaInSb Type-II Superlattice Photodiodes ......................................... 13

2.3.6 InP Based Photodiodes ........................................................................ 14

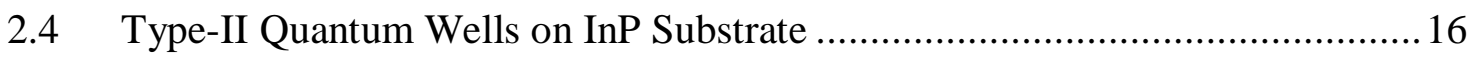

Chapter 3: $\quad$ Design of InGaAs/GaAsSb Type-II Quantum Wells Structures........19

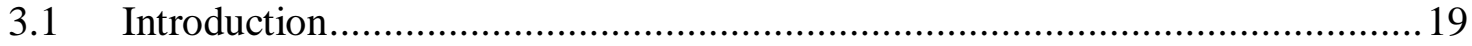

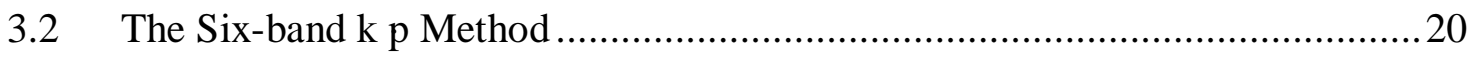

3.3 Design of InGaAs/GaAsSb Type-II Quantum Wells........................................24

3.3.1 Effects of the Thickness of the Quantum Well Layers ..............................27

3.3.2 Effects of the Composition of the Quantum Well Layers ..........................29

3.3.3 Strain Compensated InGaAs/GaAsSb MQWs .............................................33

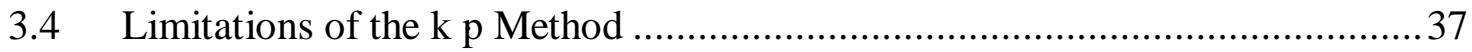

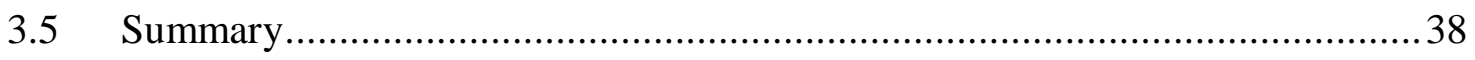

Chapter 4: Photodiode Characterization ........................................................................39

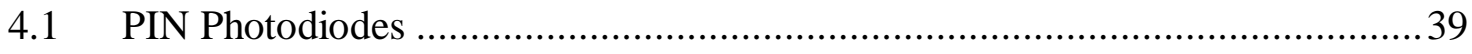




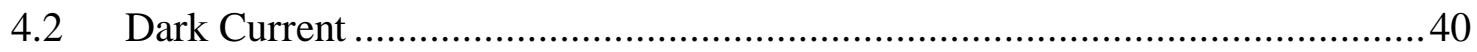

4.2.1 Diffusion Current ............................................................................. 41

4.2.2 Generation-recombination Current ........................................................

4.2.3 Band to Band Tunneling Current ..........................................................42

4.2.4 Trap-assisted Tunneling Current.............................................................43

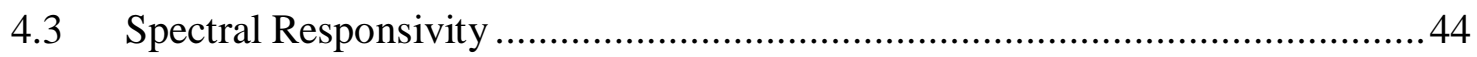

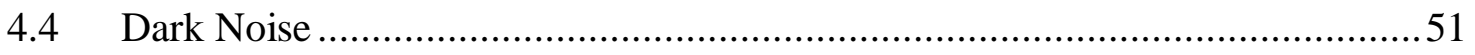

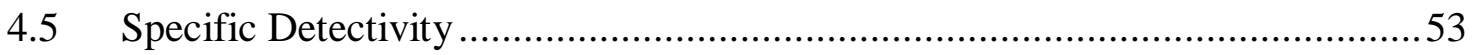

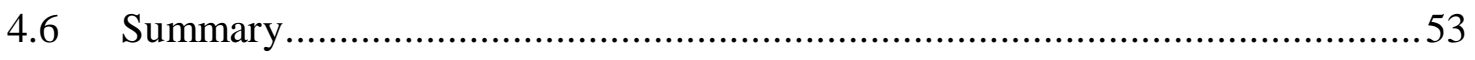

Chapter 5: InGaAs-GaAsSb Type-II Quantum Wells PIN Photodiodes .............54

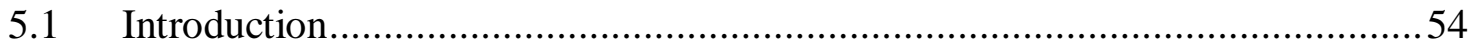

5.2 Lattice Matched Quantum Wells Photodiode (Sample A) ...............................55

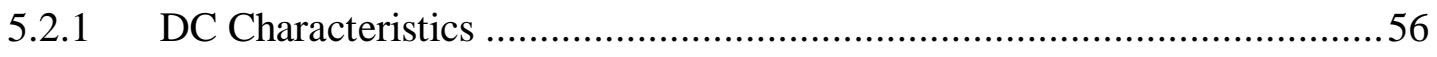

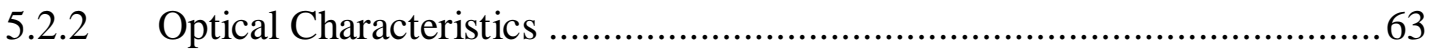

5.3 Strain Compensated Quantum Wells Photodiode (Sample B) ..........................72

5.3.1 DC Characteristics ................................................................................

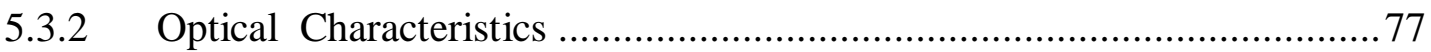

5.4 New Strain Compensated Quantum Wells Photodiode (Sample C) ...................85

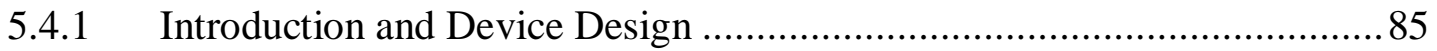

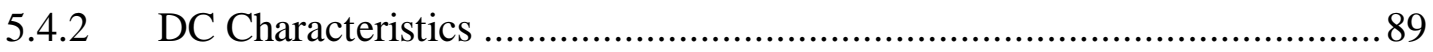

5.4.3 Optical Characteristics ........................................................................

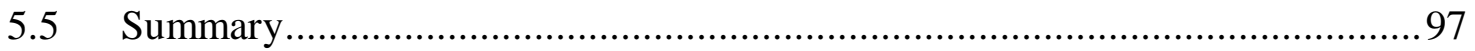

Chapter 6: Carrier Dynamics Study of InP based PIN Photodiodes with InGaAs/GaAsSb Type-II Quantum Wells........................................................................99

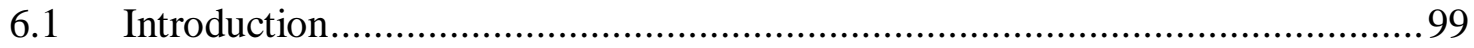

6.2 Carrier Collection Efficiency Modeling …………........................................ 100

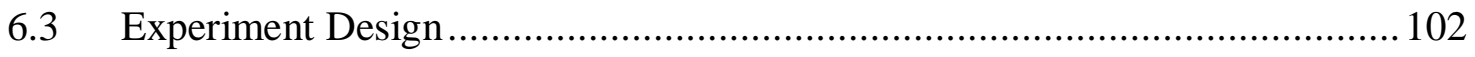

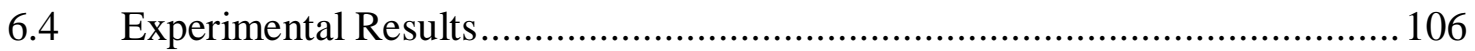

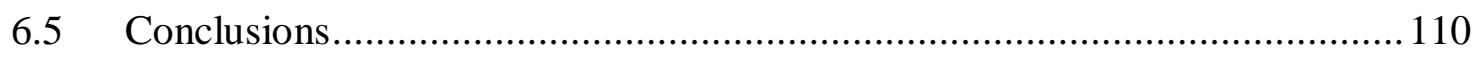

Chapter 7: Conclusions and Future Work .................................................................111

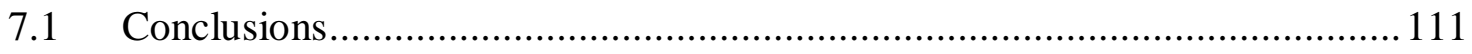


7.2 Future Work................................................................................. 113

7.2.1 Optimize the Structure of New Strain Compensated Sample.................. 113

7.2.2 InGaAs(N)/GaAsSb Type-II Quantum Wells for MWIR Detection ........ 114

7.2.3 Resonant Cavity Enhanced (RCE) Photodiodes ................................. 115

7.2.4 Heterogeneous Integration of Type-II p-i-n Photodiodes on a Silicon-onInsulator Waveguide Circuit .................................................................... 116

Appendix A: Process Flows...................................................................................... 118

Appendix B: Publications............................................................................. 122

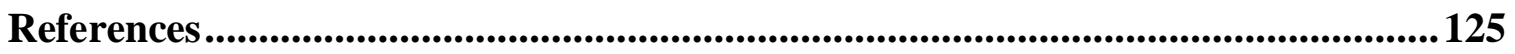

Vita.......................................................................................................................... 142 


\section{Table of Figures}

Figure 1.1 The electromagnetic spectrum. This figure is taken from Reference [1] ......... 1 Figure 1.2 Transmission of the atmospheric gases in the infrared region. This figure is

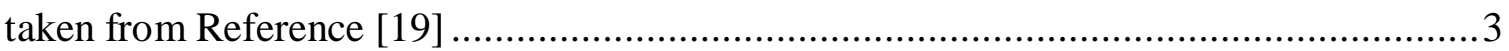

Figure 2.1 Detectivites of various infrared detectors at different operating temperatures.

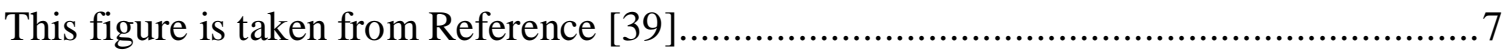

Figure 2.2 Band gap versus lattice-constant of III-V and II-VI materials. This figure is

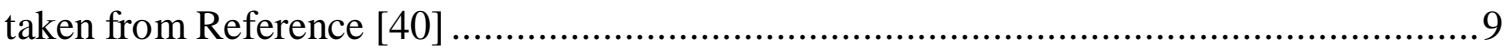

Figure 2.3 Comparisons between inter-band and inter-subband transitions .................. 11

Figure 2.4 Energy bands of type II indium arsenide/gallium antimonide (InAs/GaSb) superlattices (T2SLs). (a) Spatial band alignment in T2SL (red regions represent InAs forbidden gap and green regions represent $\mathrm{GaSb}$ forbidden gap). (b) Band structure with direct band gap and absorption process in reciprocal space (k-space). E1: First conduction band level. HH1: First heavy hole (positive charge carrier) level. LH1: First light hole level. Eg: Energy gap. This figure is taken from Reference [52] ............................. 13 Figure 2.5 Performance comparison of different InP based $\operatorname{In}_{\mathrm{x}} \mathrm{Ga}_{1-\mathrm{x}} \mathrm{As}$ photodiodes. This

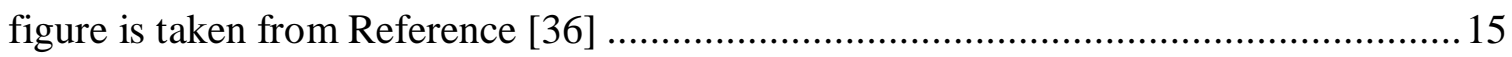

Figure 2.6 Band alignment of type-I and type-II quantum wells ............................. 17 Figure 2.7 Specific detectivity results of Sidhu's initial photodiodes compared to other infrared devices [36] 18

Figure 3.1 First Brillouin zone of FCC lattice showing symmetry labels for high symmetry lines and points. This figure is taken from Reference [82] 
Figure 3.2 Schematic diagrams for the band alignment and the wave function components for the InGaAs/GaAsSb type-II quantum well .......................................................25

Figure 3.3 Subband energy for the 5nm InGaAs/5nm GaAsSb lattice matched type-II quantum well on InP substrate .26

Figure 3.4 (a) Transition wavelength and (b) wave-function overlap dependence on the thickness change of lattice-matched InGaAs or GaAsSb. The simulation was carried out by changing the layer thickness of one material while keeping the layer thickness of the other at $5 \mathrm{~nm}$. .28

Figure 3.5 Wave function overlap versus transition wavelength by changing the layer thickness of one material while keeping the layer thickness of the other material fixed at $5 \mathrm{~nm}$ 29

Figure 3.6 Band edges changes for III-V semiconductor under compressive strain, no strain, and tensile strain 30

Figure 3.7 The dependence of the transition wavelength and the wave-function overlap versus (a) the In composition change while keeping GaAsSb lattice-matched to InP and (b) the Sb composition change while keeping InGaAs lattice-matched to InP .31 Figure 3.8 Schematic diagram of the band alignment for a InGaAs/GaAsSb type-II QW about how the $\mathrm{CB}$ of InGaAs and $\mathrm{HH}$ of GaAsSb move with increase of In composition (x) in InGaAs and $\mathrm{Sb}$ composition (y) in GaAsSb. .32

Figure 3.9 Strain-balanced multi-quantum wells consist of compressive-strained layer and tensile-strained layer. .33 
Figure 3.10 Under the strain compensation condition, the In composition in InGaAs versus $\mathrm{Sb}$ composition in GaAsSb with layer thickness of $5 \mathrm{~nm}$ for both $\mathrm{InGaAs}$ and GaAsSb layers.

Figure 3.11 Under the strain compensation condition, the transition wavelength and the wave-function overlap versus $\mathrm{Sb}$ composition change with layer thickness of $5 \mathrm{~nm}$ for both InGaAs and GaAsSb layers .36

Figure 3.12 Under the strain compensation condition, wave function overlap versus transition wavelength for different thickness combinations for both InGaAs and GaAsSb layers with $\mathrm{Sb}$ composition changing from 0.3 to 0.9 .37

Figure 4.1 Device structure and electrical field distribution of PIN photodiodes 39

Figure 4.2 Principle of FTIR operation .45

Figure 4.3 The spectrum of the infrared light measured by the DTGS detector .46

Figure 4.4 The spectrum of the infrared light measured by the external detector..... .47

Figure 4.5 The relative responsivity of the external detector to be tested .48

Figure 4.6 The spectral distribution of energy in the black body radiation at temperature of $700{ }^{\circ} \mathrm{C}$ .50

Figure 5.1 A schematic of the PIN device structure with type-II $7 \mathrm{~nm} \operatorname{In}_{0.53} \mathrm{Ga}_{0.47} \mathrm{As} / 5 \mathrm{~nm}$ $\mathrm{GaAs}_{0.5} \mathrm{Sb}_{0.5}$ MQWs as absorption layer (Sample A) .55

Figure 5.2 HRXRD data of Sample A with 100 pairs of $7 \mathrm{~nm} \operatorname{In}_{0.53} \mathrm{Ga}_{0.47} \mathrm{As} / 5 \mathrm{~nm}$ $\mathrm{GaAs}_{0.5} \mathrm{Sb}_{0.5} \mathrm{MQWs}$ .56

Figure 5.3 Dark current versus voltage for the $180 \mu \mathrm{m}$ diameter Sample A measured at different temperatures. 
Figure 5.4 Dark current versus device area for lattice matched Sample A with bias of $0.5 \mathrm{~V}$ measured at $290 \mathrm{~K}$ .58

Figure 5.5 Arrhenius plot of the dark current for $180 \mu \mathrm{m}$ diameter Device A at reverse bias $-0.5 \mathrm{~V}$. .59

Figure 5.6 $\mathrm{R}_{0} \mathrm{~A}$ product for $180 \mu \mathrm{m}$ diameter Sample A at different temperatures..... 60

Figure 5.7 Experimental and simulated current considering diffusion, GR, band-to-band direct tunneling and trap-assisted tunneling for the lattice matched InGaAs-GaAsSb MQW photodiode with $130 \mu \mathrm{m}$ diameter investigated at (a)290 K, (b)260K, (c) 230K, (d) 200K. And the diffusion component is too low to be shown in the same plot.

Figure 5.8 Normal incident photo response of the device under different temperatures, at reverse bias of $-0.5 \mathrm{~V}$ for Sample A.

Figure 5.9 Blackbody responsivity versus temperature under different reverse bias for Sample A .65

Figure 5.10 Dark noise spectrum densities versus temperature under bias of $-0.5 \mathrm{~V}$ for Sample A 67

Figure 5.11 Detectivity of Sample A at different temperatures under reverse bias of $-0.5 \mathrm{~V}$

Figure 5.12 Comparison of the detectivities of Sample A at $2.4 \mu \mathrm{m}$ (triangle) with that of Sidhu's device (dot)[34] and other infrared detectors [36] .68

Figure 5.13Angular dependence of normalized blackbody responsivity of Sample A under zero bias

Figure 5.14 (a) Optical transient response of $100 \mu \mathrm{m}$ photodiode under bias of $-0.1 \mathrm{~V}$ (b) Fourier transform of the pulsed transient response of $100 \mu \mathrm{m}$ photodiode .71 
Figure 5.15 A schematic of the PIN device structure with type-II $7 \mathrm{~nm} \operatorname{In}_{0.34} \mathrm{Ga}_{0.66} \mathrm{As} / 5 \mathrm{~nm}$ $\mathrm{GaAs}_{0.25} \mathrm{Sb}_{0.75}$ MQWs as absorption layer. .72

Figure 5.16 HRXRD data of Sample $B$ of 100 pairs of $7 \mathrm{~nm} \operatorname{In}_{0.34} \mathrm{Ga}_{0.66} \mathrm{As} / 5 \mathrm{~nm}$ $\mathrm{GaAs}_{0.25} \mathrm{Sb}_{0.75}$ .73

Figure 5.17 Dark current versus voltage for a $180 \mu \mathrm{m}$ diameter device measured at different temperatures. .74

Figure 5.18 Dark current versus device area for lattice matched Sample B with bias of $0.5 \mathrm{~V}$ measured at $290 \mathrm{~K}$ .75

Figure 5.19 Arrhenius plot of the dark current for $180 \mu \mathrm{m}$ diameter Device B at reverse bias $-0.5 \mathrm{~V}$ 76

Figure 5.20 $\mathrm{R}_{0} \mathrm{~A}$ for Sample $\mathrm{B}$ and Sample A with $180 \mu \mathrm{m}$ diameter at different temperatures. .77

Figure 5.21 Normal incident photo response of the device under different temperatures, at reverse bias of $-0.5 \mathrm{~V}$ for Sample B .78

Figure 5.22 Blackbody responsivity versus temperature under different reverse bias for Sample B. .79

Figure 5.23 Capacitance-Voltage (C-V) measurements of the $180 \mu \mathrm{m}$ diameter devices for both Sample A and Sample B .80

Figure 5.24 Dark noise spectrum densities versus temperature under reverse bias of $0.5 \mathrm{~V}$ for Sample B and Sample A .80

Figure 5.25 Detectivity of Sample B at different temperatures under reverse bias of $-0.5 \mathrm{~V}$ 
Figure 5.26 Comparison of the detectivities of Sample B at $2.7 \mu \mathrm{m}$ and $3 \mu \mathrm{m}$ (star) with that of Sidhu's device (dot) [34], Sample A at $2.4 \mu \mathrm{m}$ (triangle) and various other infrared detectors

Figure 5.27 Noise versus reverse bias for a 180 diameter Sample B measured at room temperature

Figure 5.28 Blackbody responsivity and blackbody detectivity of Sample B versus reverse bias for a 180 diameter device measured at room temperature .84

Figure 5.29 (a) the structure of conventional strain compensated MQWs, (b) the structure of new strain compensated MQWs .86

Figure 5.30 Transition wavelength v.s Sb composition of $5 \mathrm{~nm}$ InGaAs $/ 5 \mathrm{~nm}$ GaAsSb type-II quantum well with difference net compressive strain. 87

Figure 5.31 Schematic diagram for Sample C .88

Figure 5.32 HRXRD data of Sample C of 20 pairs of type-II quantum wells .89 Figure 5.33 Dark current of Sample C versus voltage with $130 \mu \mathrm{m}$ diameter measured at different temperatures. 90

Figure 5.34 Arrhenius plots of the dark current at $-0.5 \mathrm{~V}$ and the $\mathrm{R}_{0} \mathrm{~A}$ product for $130 \mu \mathrm{m}$ diameter Sample C

Figure 5.35 Normal incident photo response of the sample $\mathrm{C}$ at different temperatures (a) with zero reverse bias, (b) with $0.5 \mathrm{~V}$ reverse bias 92

Figure 5.36 Blackbody responsivity of Sample C under different bias versus temperature

Figure 5.37 Band Alignment of the new strain compensated quantum wells in Sample C 
Figure 5.38 Dark noise spectrum densities versus temperature under $0 \mathrm{~V}$ bias and $-0.5 \mathrm{~V}$ bias for Sample C

Figure 5.39 Detectivities of Sample C versus wavelength under 0V bias and $-0.5 \mathrm{~V}$ bias compared with that of Sample B at $290 \mathrm{~K}$ 96

Figure 6.1 InP based type-II Quantum well photodiodes structure for carrier study (a) Sample D with a 50nm InP barrier (b) Sample E with a 50nm GaAsSb barrier 102 Figure 6.2 (a) Carrier transport in type-II quantum well structure in sample D if phononassisted tunneling dominates (b) Carrier transport in type-II quantum well structure in sample E if phonon-assisted tunneling dominates

Figure 6.3 (a) Carrier transport in type-II quantum well structure in sample D if thermionic emission dominates (b) Carrier transport in type-II quantum well structure in sample $\mathrm{E}$ if thermionic emission dominates

Figure 6.4 the carrier collection efficiency as a function of the ratio between phononassist tunneling time and the thermionic emission time 106

Figure 6.5 HRXRD $\omega / 2 \theta$ of sample D and sample E with 100 pairs of $7 \mathrm{~nm}$ $\mathrm{In}_{0.53} \mathrm{Ga}_{0.47} \mathrm{As} / 5 \mathrm{~nm} \mathrm{GaAs} 0.5 \mathrm{Sb}_{0.5}$

Figure 6.6 Dark current of $350 \mu \mathrm{m}$ devices for each structure at room temperature ...... 108 Figure 6.7 (a) Normal incident photo response of the device at different temperatures, at reverse bias of $-1 \mathrm{~V}$ for Device A. (b) Normal incident photo response of the device at different temperatures, at reverse bias of $-1 \mathrm{~V}$ for Device B. (c) the photo responses of both devices at $200 \mathrm{~K}$ on the same plot 
Figure 7.1 Comparison between the detectivities of Sidhu's device (dot), Sample A (triangle), Sample B (5-point star) and Sample C (7-point star) with that of various other

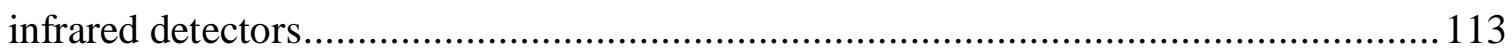

Figure 7.2 Transition wavelength as a function of $\mathrm{N} \%$ in the InGaAsN layer ...............115

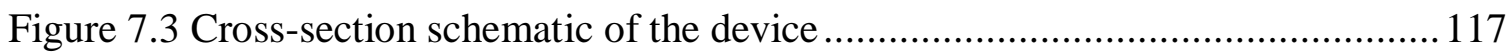




\section{List of Tables}

Table 5-1 Equations used to model the dark current of bulk PIN photodiodes ...............61

Table 5-2 Parameters used to fitting the lattice matched InGaAs/GaAsSb p-i-n

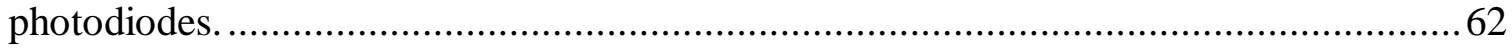

Table 5-3 Performance of different InP based type-II Quantum wells Photodiodes ........97 


\section{Chapter 1: Introduction}

Infrared (IR) radiation is the electromagnetic waves with wavelength extending from the red edge of the visible spectrum at 750 nanometers $(\mathrm{nm})$ to 300 micrometers $(\mu \mathrm{m})$ as shown in Figure 1.1 [1]. This range of wavelengths corresponds to the photon energy ranging from 1.24 milli-electron volts $(\mathrm{meV})$ to 1.7 electron volts $(\mathrm{eV})$, and includes most of the thermal radiation emitted by objects at room temperature. The existence of infrared radiation was first discovered by William Herschel [2], who found infrared radiation in sunlight by passing the light through a prism and holding a thermometer beyond the red end of the visible spectrum.

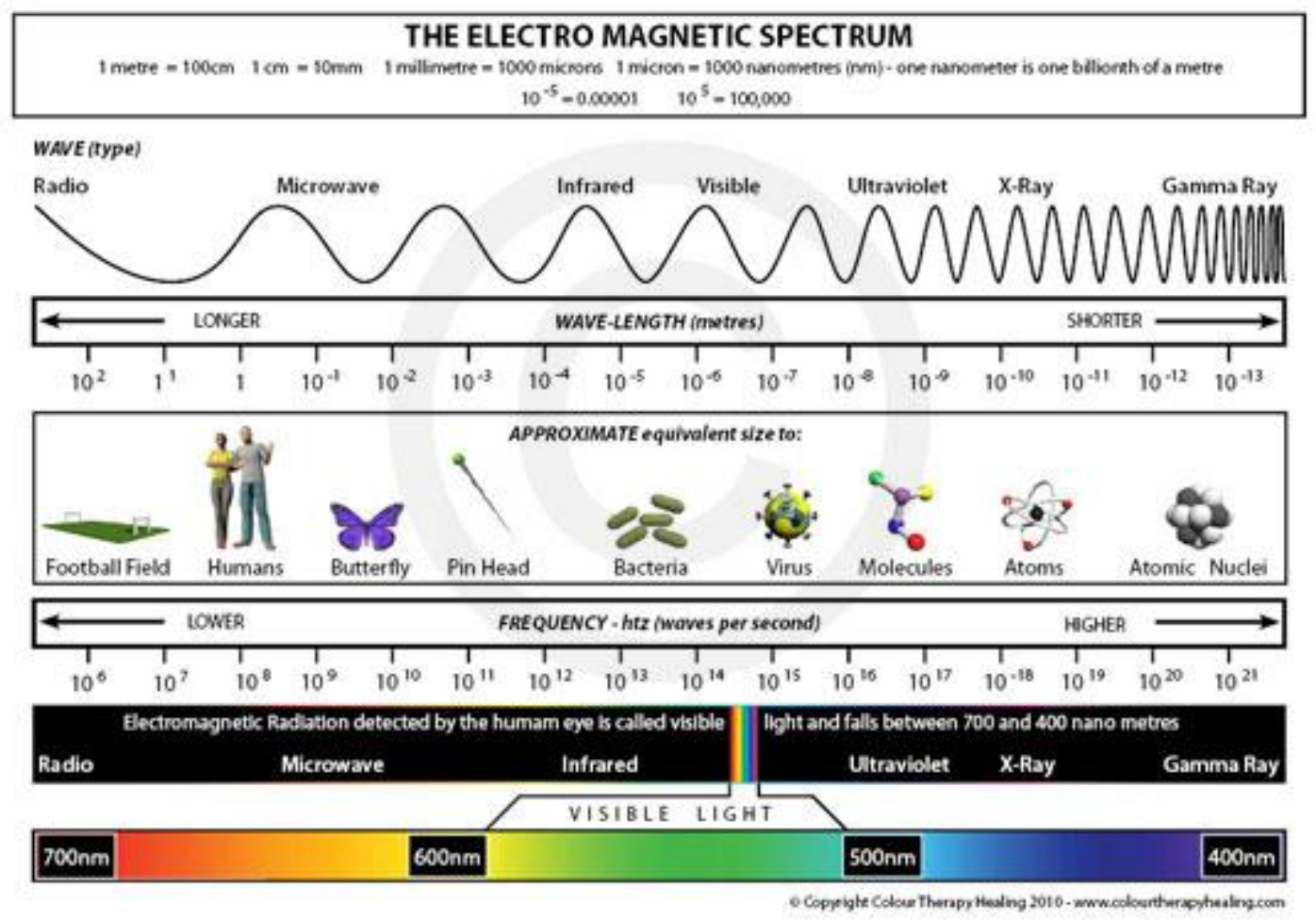

Figure 1.1 The electromagnetic spectrum. This figure is taken from Reference [1] 


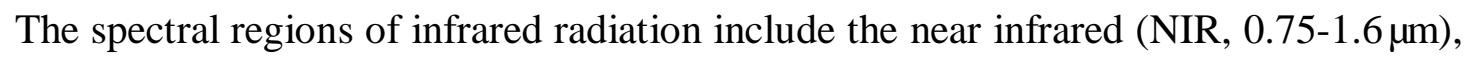

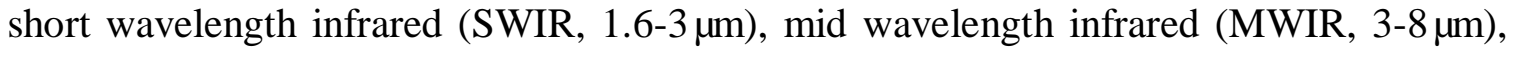

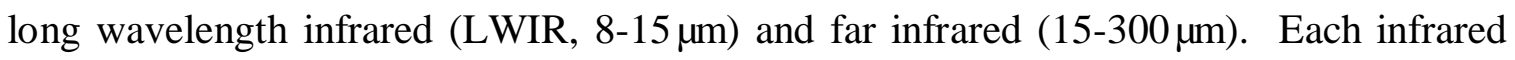
spectral region can be used for different applications. For example, NIR is commonly used in fiber optical telecommunication because of low optical attenuation losses in the silica medium[3, 4], while LWIR can be used for thermal imaging [5, 6]. SWIR and MWIR infrared radiation also have many applications in various areas [7-18], which will be discussed below. In this dissertation, I will mainly focus on the SWIR and MWIR regions.

The high atmospheric transmission in MWIR region (shown in Figure 1.2 [19]) makes these wavelengths attractive for ground-based astronomical observations and imaging [20]. Moreover, organic compounds often have vibrational energies located in the SWIR and MWIR regions, and many chemical compounds such as nitrous oxide $\left(\mathrm{N}_{2} \mathrm{O}\right)$, ammonia $\left(\mathrm{NH}_{3}\right)$, methane $\left(\mathrm{CH}_{4}\right)$ and carbon dioxide $\left(\mathrm{CO}_{2}\right)$, also have absorption line in the SWIR and MWIR regions $[15,18]$. As a result, these two infrared regions are essential in applications such as gas sensing, sample composition analysis and environmental control. Non-invasive blood glucose determination and biomedical imaging $[7,10-12,21]$ are examples of the medical diagnostic applications of SWIR and MWIR light. Therefore, SWIR and MWIR photodetectors with good performance are highly desirable, which have inspired significant research in this field. 


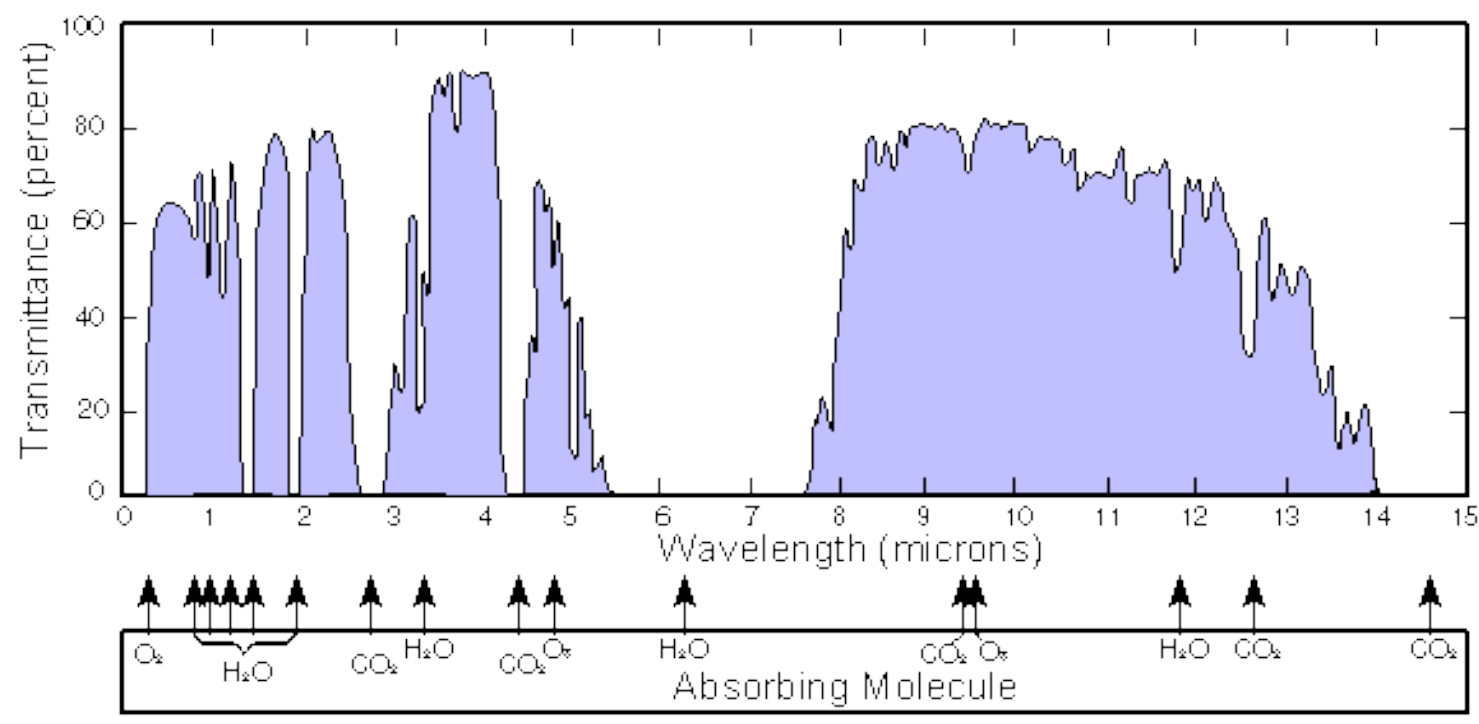

Figure 1.2 Transmission of the atmospheric gases in the infrared region. This figure is taken from Reference [19]

The requirements of a good photodetector include high sensitivity, low dark current, low noise, and high operating temperature, ideally close to room temperature. Mercury Cadmium Telluride (HgCdTe) is the predominant material system used in SWIR, MWIR and LWIR applications [22-26]. However, the HgCdTe material system often suffers from poor material uniformity, difficult material growth, device instability, high cost and low yield [27]. The lack of uniformity also degrades the performance of focal plane arrays. These disadvantages of $\mathrm{HgCdTe}$ photodetectors have led to research efforts investigating alternative technologies, such as quantum well infrared photodetectors [28], quantum dot infrared photodetectors (QDIPs) [29-31] and InAs/GaSb type-II superlattices photodetectors [32, 33]. However, photodetectors using these alternative technologies also have their own shortcomings, which will be investigated in the next chapter. 
InP based Photodiodes with InGaAs/GaAsSb type-II quantum wells absorption regions are promising candidates for SWIR and MWIR detection, especially at operating temperatures close to ambient [34]. These devices can benefit from the mature InP material and device technologies. Unlike In-rich lattice mismatched InGaAs on InP substrate, the generation of crystal defects by lattice mismatch can be eliminated in this material system, since these quantum well structures can be grown on InP substrate using lattice matched or strain compensated materials. Therefore, low dark currents can be expected for those devices.

The goal of this study is to exploit the advantages of the InP based type-II quantum wells photodiodes for high performance SWIR and MWIR detectors at high operating temperatures $(\geq 200 \mathrm{~K})$ where devices can be operated uncooled or with a thermoelectric cooler. In this dissertation, the performance of the InP based type-II quantum wells PIN photodiodes for SWIR and MWIR detection will be studied.

The dissertation is organized as follows: Chapter 2 will discuss the alternative technologies of infrared photodetectors and introduce the approach that I have pursued: InGaAs/GaAsSb type-II quantum wells on InP substrates. In Chapter 3, I will discuss the design of InGaAs/GaAsSb type-II quantum wells structures in order to achieve good photodetector performance. Additionally, the $\mathrm{k} \cdot \mathrm{p}$ method, as the theoretical foundation for designing quantum well structure, will be introduced in Chapter 3 as well. Next in Chapter 4, the various figures of merits (dark current, quantum efficiency, dark noise, specific detectivity, etc.) used to qualify SWIR and MWIR photodiode performance will be discussed. Then in Chapter 5, I will focus on the experimental performance of three different designs of InGaAs/GaAsSb type-II quantum well photodiodes on InP substrate. 
The results of electrical and optical characterization will be reported, and various performance-related issues will be discussed. In Chapter 6, the carrier dynamics of InGaAs/GaAsSb type-II quantum well structures will be studied, in order to improve the carrier collection efficiency. Finally, Chapter 7 will summarize the major accomplishments and discuss potential research areas that can be further looked upon and implemented to improve the performance of InP based photodetectors with SWIR and MWIR response. 


\section{Chapter 2: Overview of Infrared Semiconductor}

\section{Detectors}

\subsection{Introduction}

In April of 1800, William Herschel wrote, "Thermometer No.1 rose 7 degrees in 10 minutes by an exposure to the full red coloured rays, I drew back the stand...thermometer No. 1 rose in 16 minutes, 8.75 degrees when its centre was $1 / 2$ inch out of the visible rays.", which demonstrated the existence of radiation beyond the red end of the visible spectrum [2]. Later, it was discovered experimentally that every object emits radiation with a range of wavelengths that depends on the temperature of that specific object. The relationship between temperature and the spectrum distribution of emission wavelengths was first described accurately and empirically by Max Planck in 1900 [35].

The thermometers William Herschel used were a primitive type of infrared detector. In the remaining sections of this chapter, I describe several different material systems and technologies for infrared detection.

\subsection{Types of Infrared Detectors}

Infrared detectors are generally classified into thermal and photon detectors. Both types of detectors respond to absorbed photons, but have different response mechanisms, which then lead to variations in performance related to speed, spectral responsivity, specific detectivity and operation temperature. These figures of merit will be discussed in detail in Chapter 4. Figure 2.1 shows the specific detectivity (a measure of photodetector sensitivity) versus wavelength for a number of commercially available IR detectors, 
along with their respective operating temperature. Detailed information on the different infrared detector technologies is available in reference [36-39].

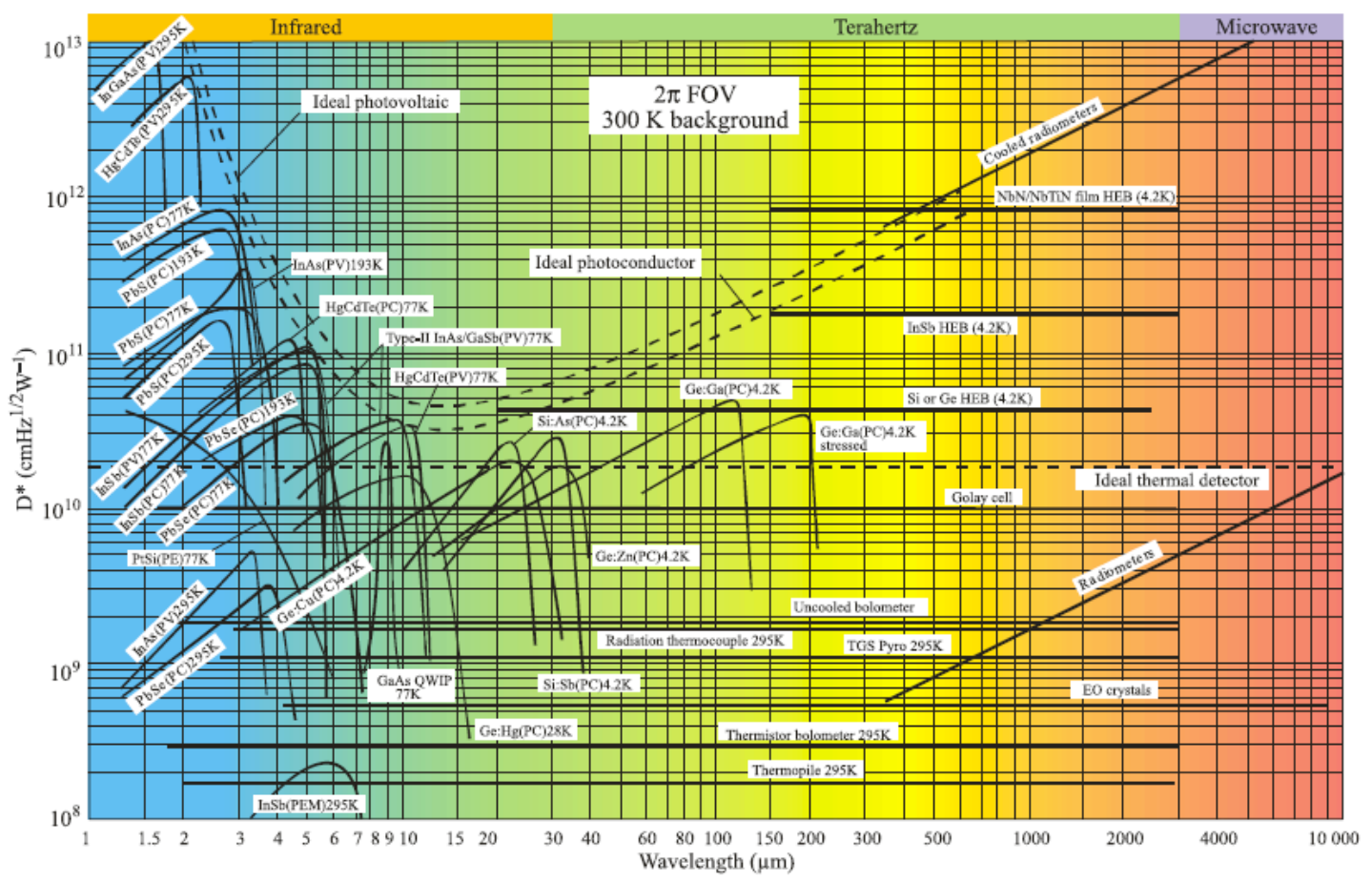

Figure 2.1 Detectivites of various infrared detectors at different operating temperatures. This figure is taken from Reference [39]

\subsubsection{Thermal Detectors}

In thermal detectors, the incident radiation is absorbed to change the material's temperature, and the temperature change can be measured by some temperature dependent mechanisms, such as thermoelectric voltage, resistance and pyroelectric voltage [36]. Usually, the output signal of a thermal detector does not depend on the photonic nature of the incident radiation. Therefore, the response is generally wavelength independent; the signal depends upon the radiant power but not its spectral content. This is based on the assumption that the mechanism of the absorption of the radiation itself is wavelength independent, which may not be true in certain instances. Examples of thermal 
detectors include thermopiles, resistive bolometers and pyroelectric detectors. Thermal detectors are usually light in weight, rugged, reliable, and low in cost. Moreover, thermal detectors have the advantage of room temperature operation. However, these detectors are slow (response times on the order of ms), and less sensitive at high frequencies [36].

\subsubsection{Photon Detectors}

The disadvantages of thermal detectors have driven research and development efforts into alternative infrared detection systems, namely photodetectors based on semiconductor materials. The photon detectors generally respond to incident radiation on an electronic level. It is not necessary to heat the entire device as thermal detectors, so photon detectors are much faster than thermal detectors. There are two basic forms of semiconductor photodetectors: photoconductive (PC) and photovoltaic (PV) detectors. Photoconductive detectors are made of materials with low conductivity. The conductivity increases with incident radiation intensity because of the photo generated carriers, and this leads to an increased current when voltage is applied across the detector. Photovoltaic detectors are diodes that produce a voltage across the terminals when photons are absorbed; the potential difference due to light illumination can cause current flow proportional to incoming light intensity.

\subsection{Material Systems for MWIR Photodetector}

Different semiconductor materials, including the group-IV, III-V compounds, II-VI compounds and IV-VI compounds, have been used to make different types of photodetectors. Figure 2.2 shows the band gap versus lattice constant diagram for the III$\mathrm{V}$ and the II-VII material systems. A brief description of the primary material systems 
and detector technologies used for MWIR detection will be presented in the following section.

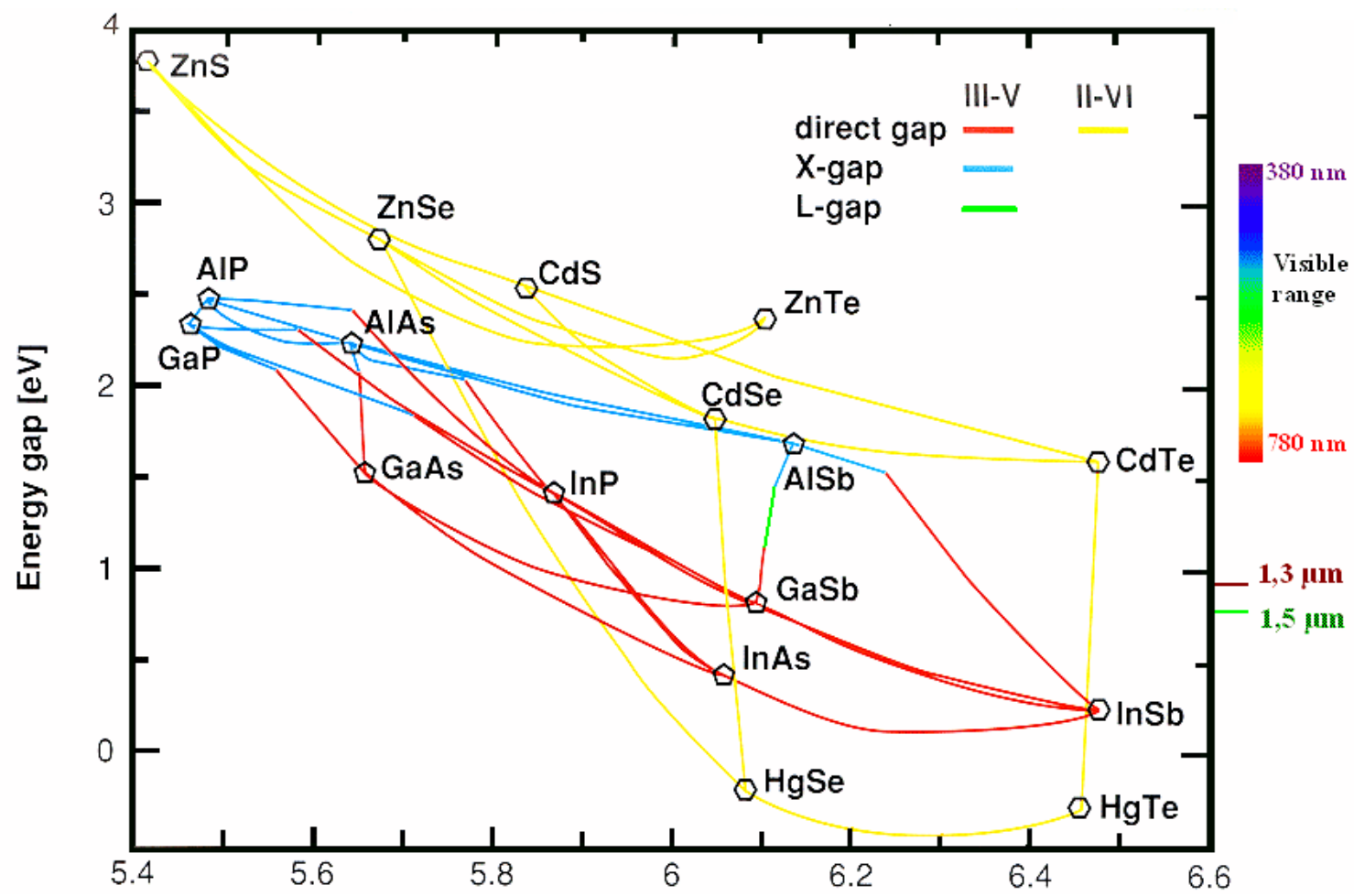

Figure 2.2 Band gap versus lattice-constant of III-V and II-VI materials. This figure is taken from Reference [40]

\subsubsection{IV-VI material systems}

The lead-chalcogenide materials ( $\mathrm{PbS}, \mathrm{PbSe}, \mathrm{PbSnTe})$ were used as infrared detectors from late 1960s until mid 1970s. These materials are easier to prepare and more stable compared to $\mathrm{HgCdTe}$ detector (discussed in the next section). However, they suffer from two significant drawbacks [36]. First, they have very large relative permittivity depending on temperature and illumination, which will result in high diode capacitance and thus limited frequency response. The other drawback is their high thermal coefficient of expansion, which limits their applicability in hybrid configurations with silicon multiplexers [36]. 


\subsubsection{II-VI material systems}

$\mathrm{HgCdTe}$ is one of the most developed materials used as infrared detectors. The band gap of $(\mathrm{HgTe})_{x}(\mathrm{CdTe})_{1-\mathrm{x}}$ alloys can be tailored from $1 \mu \mathrm{m}$ to $30 \mu \mathrm{m}$ by changing the composition [25, 41]. HgCdTe photodetectors absorb the IR radiation across the fundamental energy gap, and have high quantum efficiency due the large optical absorption coefficient. These detectors also have relatively low thermal generationcombination rate which results in a higher operation temperature compared to other approaches.

However, $\mathrm{HgCdTe}$ has serious technological problems in mass production due to its weak $\mathrm{Hg}$-Te bond [41]. Moreover, $\mathrm{HgCdTe}$ detectors suffer from poor material uniformity, low yield, high cost in growth and processing, and surface instability [27, 41]. These material difficulties have made it necessary to examine the performance of alternative material systems.

\subsubsection{GaAs and InP Based QWIPs and QDIPs}

Quantum well infrared photodetectors (QWIPs) and quantum dot infrared photodetectors (QDIPs) use inter-subband (intra-band) absorption mechanism involving transitions within the same band, rather than inter-band absorption, which involves transitions between the conduction and valence bands [42]. Figure 2.3 compares the interband and intra-band transitions. Unlike the inter-band transitions, the quantum wells or quantum dots must be doped to make the inter-subband transition possible. The infrared absorption mechanism involves the transition of an electron (or hole) from the doped quantum well or quantum dot ground state in the conduction (or valence) band to an unoccupied excited state in the same band. 


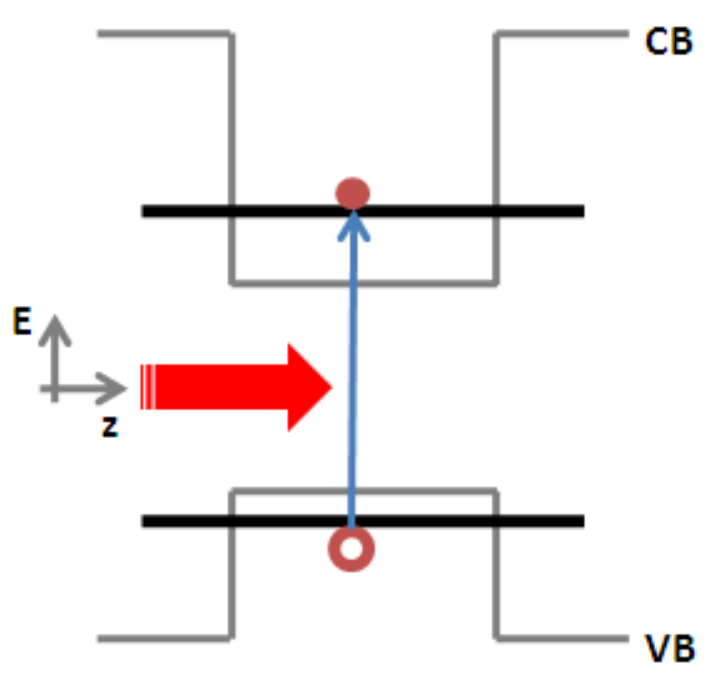

Inter-band Transition

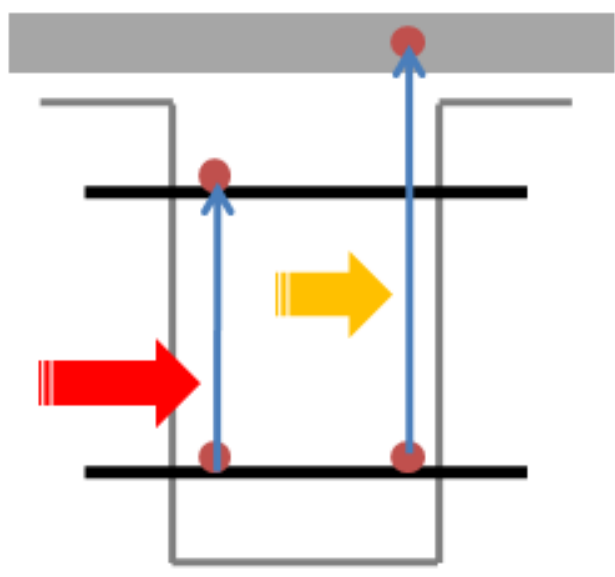

Inter-subband Transition

Figure 2.3 Comparisons between inter-band and inter-subband transitions

QWIPs have the advantages of mature material growth and processing technologies, which lead to high uniformity and excellent reproducibility [5, 6, 28, 43]. A disadvantage for QWIPs is low quantum efficiency. For n-doped QWIPs, normal incidence absorption is forbidden, due to the quantum mechanical selection rule in the $\Gamma$-symmetry conduction band [28, 42]. An efficient optical coupling scheme (e.g., using diffraction gratings or random gratings) is required for normal incidence detection [28]. This problem can be partially solved by using p-doped QWIPs [42, 44-46], since the strong mixing between the light and heavy holes in the valence band at $\mathrm{k} \neq 0$, permits normal-incidence absorption. However, the quantum efficiency of p-doped QWIPs is low [45]. Furthermore, the intersubband lifetimes in both n-doped QWIP and p-doped QWIP detectors are inherently short (about 10ps), which result in relatively poor performance at temperatures $>70 \mathrm{~K}$, when thermal generated carriers dominate over optically generated carriers [47].

QDIPs [29-31], whose active regions are composed of quantum dot layers separated by barriers, show a number of potential advantages over QWIPs. First, the inter-subband 
absorption is allowed at normal incidence [42]. Due to three-dimensional (3D) confinement of the electrons, normal incident transitions are strong in QDIPs. Second, the thermal generation rate in QDIPs is smaller than that in QWIPs due to the energy quantization in all three dimensions. Phonon assisted scattering is less likely to occur because it is difficult for the energy level spacing of a quantum dot to be equal to that of the phonon [48]. This prohibition does not apply to the quantum wells, since the levels are quantized only in the growth direction and a continuum already exists in the other two directions. Therefore, the dark current of QDIPs is expected to be lower than that of QWIPs and bulk detectors such as HgCdTe. However, the design and growth of quantum dots is complicated and challenging in order to achieve the expected performance. Experimentally, operation of most QDIPs requires cryogenic cooling to lower the temperature [30].

\subsubsection{InSb and InAs Based Photodetectors}

$\mathrm{InSb}$ is the narrowest band gap III-V binary material. Bulk InSb based device with a cut-off wavelength around $5 \mu \mathrm{m}$ is another option for MWIR detection, since it can provide higher uniformity than $\mathrm{HgCdTe}$. However, the dark current performance of InSb based photodiode degrades significantly at room temperature [49], whereas, better performance can be obtained from $\mathrm{HgCdTe}$ photodetectors [36]. InAs is similar to InSb, but has a larger energy band gap, so the cut-off wavelength is shorter (around $3.6 \mu \mathrm{m}$ ). The dark current performance of InAs based photodiode also degrades significantly at room temperature [50]. Moreover, for imaging applications, InAs based photodiodes require the substrate to be thinned for post hybridization to a readout integrated circuit, which is a low yield and cumbersome process. 


\subsubsection{InAs/GaInSb Type-II Superlattice Photodiodes}

InAs/GaInSb Type-II superlattices (SLSs) were first proposed by Esaki et al. in 1970s [51]. The conduction band of the InAs layer resides around $0.1 \mathrm{eV}$ below the valance band of the GaSb layer at $300 \mathrm{~K}$, as shown in Figure $2.4[52,53]$. In these superlattices, the electrons are confined in the InAs quantum wells, while the holes are confined in the GaSb quantum wells. This spatial separation of the electrons and holes results in type-II band alignment. When the thickness of the individual layers is thin enough, electrons and holes in the adjacent wells interact, resulting in a continuous energy state across different wells in the superlattice. The absorption cut-off wavelength of these SLS structures can be tuned from $2 \mu \mathrm{m}$ to $30 \mu \mathrm{m}$ by changing the layer thicknesses [54-59]. This is advantageous at long wavelengths when the growth of bulk $\mathrm{HgCdTe}$ becomes extremely difficult due to the sensitivity of the material band gap to cadmium (Cd) composition, and the change of absorption wavelength in InAs-GaInSb material systems is less sensitive to the thickness and composition of each layer. The SLSs material system is considered as an alternative candidate for new generation IR detectors.

(a)

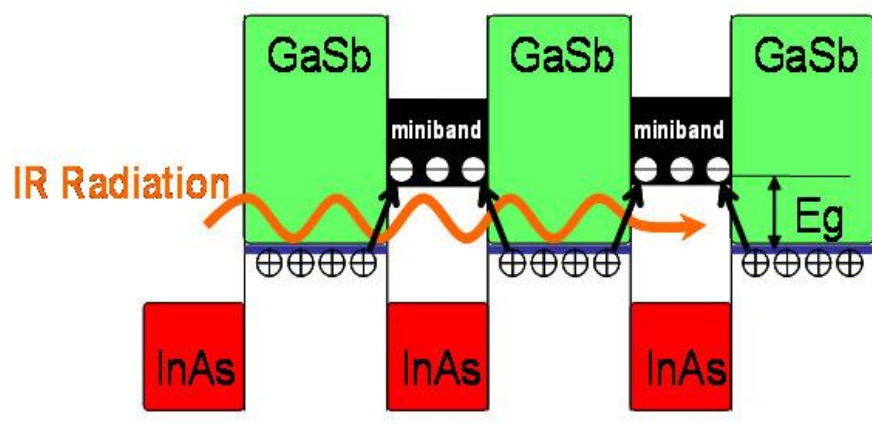

(b)

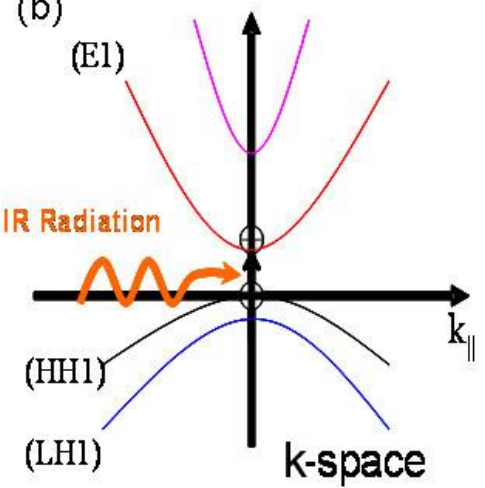

Figure 2.4 Energy bands of type II indium arsenide/gallium antimonide (InAs/GaSb) superlattices (T2SLs). (a) Spatial band alignment in T2SL (red regions represent InAs forbidden gap and green regions represent $\mathrm{GaSb}$ forbidden gap). (b) Band structure with direct band gap and absorption process in reciprocal space (k-space). E1: First conduction band level. HH1: First heavy hole (positive charge carrier) level. LH1: First light hole level. Eg: Energy gap. This figure is taken from Reference [52] 
Despite the hopeful outlook, InAs/GaInSb material system is still in the early stage of development. The material growth and device processing are immature compared to that of GaAs and InP based technologies. High performance operation is still limited to temperatures below 200K [60, 61].

\subsubsection{InP Based Photodiodes}

InP based materials have traditionally been used to make high performance photonics devices in the $1.1 \mu \mathrm{m}-1.6 \mu \mathrm{m}$ wavelength range. These devices enjoy mature $\mathrm{InP}$ substrate fabrication and epitaxial growth technology which can be manufactured on large diameter (4-inch) wafer with very low etch pit densities $\left(<100\right.$ defects $\left./ \mathrm{cm}^{2}\right)$. Using a direct band gap (type-I) absorber $\left(\operatorname{In}_{0.53} \mathrm{Ga}_{0.47}\right.$ As lattice matched to $\mathrm{InP}$ ) leads to devices which have a cut-off wavelength of $1.65 \mu \mathrm{m}$, with a very low dark current density of $1.4 \mathrm{nA} / \mathrm{cm}^{2}$ at room temperature [62].

Different technologies have been investigated to extend the detection wavelength beyond that of lattice matched $\operatorname{In}_{0.53} \mathrm{Ga}_{0.47} \mathrm{As}$. The quaternary material system, InGaAsN lattice matched to InP substrates, whose band gap reduces as a small fraction of nitrogen is incorporated, is one of the candidates for light absorption layer of such photodiodes [63-66]. However, the growth of InGaAsN is challenging and still in the early stage of development $[63,64,67]$. Highly strained $\operatorname{In}_{x} \mathrm{Ga}_{1-\mathrm{x}} \mathrm{As}(\mathrm{x}>0.53)$ quantum wells can also be used for longer wavelength detection $[68,69]$. However, it is difficult to further extend the detection wavelength beyond $2 \mu \mathrm{m}$ using this approach due to the lattice mismatch issue [70]. Longer wavelength absorption up to $2.9 \mu \mathrm{m}$ can be achieved using mismatched InGaAs on a relaxed buffer layer [71]. Hamamatsu sells an uncooled InGaAs 
relaxed device with a maximum detection wavelength of $2.6 \mu \mathrm{m}$, dark current density between 0.6 and $5 \mathrm{~mA} / \mathrm{cm}^{2}$ depending on device area, responsivity around $1.3 \mathrm{~A} / \mathrm{W}$, and a specific detectivity of $5 \times 10^{10} \mathrm{~cm} \cdot \mathrm{Hz}^{1 / 2} \cdot \mathrm{W}^{-1}$ [72]. However, these devices suffer from crystal defects that propagate into the active region and leads to high dark currents and low yield for array type device fabrication. Figure 2.5 shows the comparative room temperature performance of various InP based photodiodes [36].

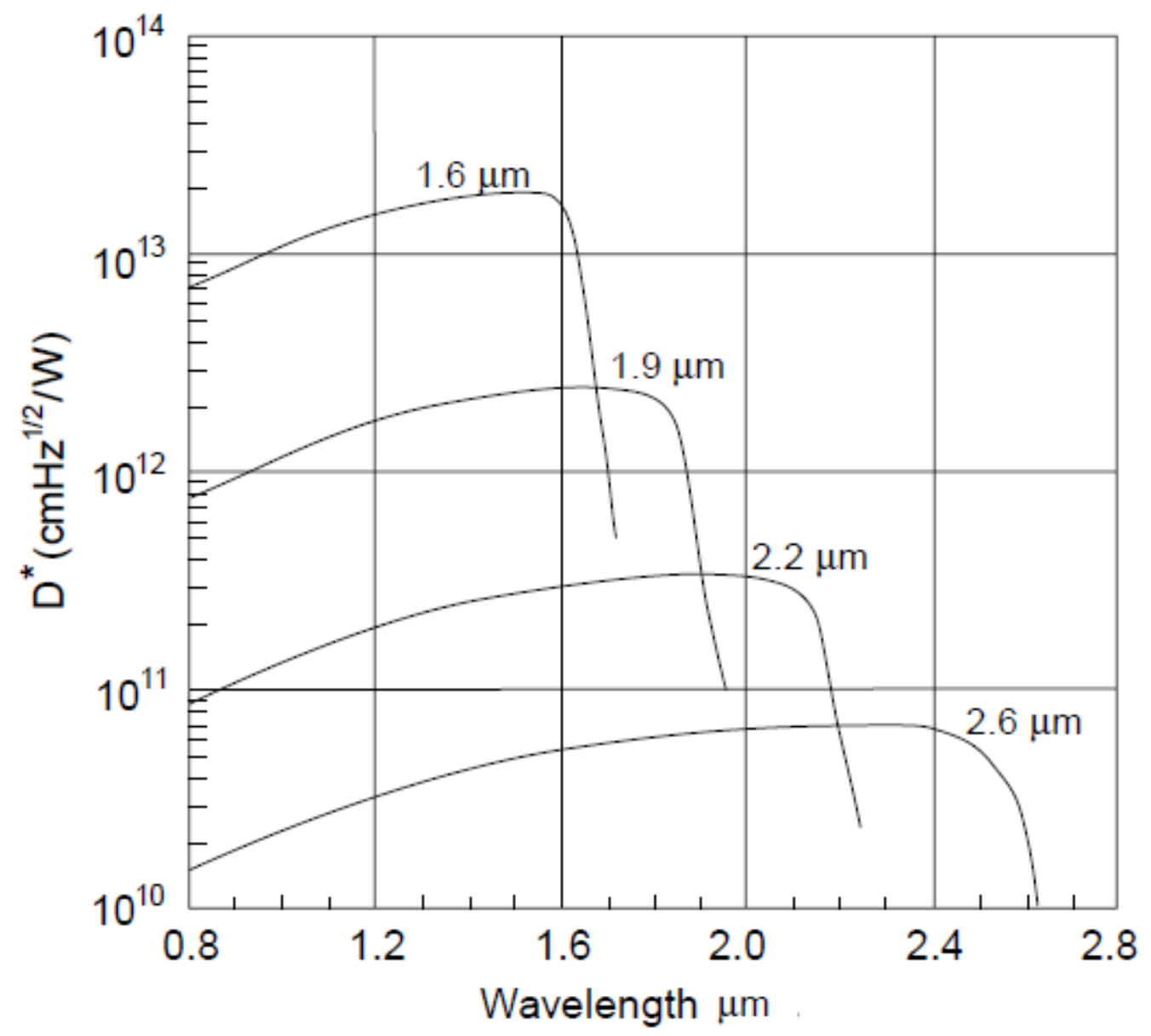

Figure 2.5 Performance comparison of different $\mathrm{InP}$ based $\mathrm{In}_{\mathrm{x}} \mathrm{Ga}_{1-\mathrm{x}} \mathrm{As}$ photodiodes. This figure is taken from Reference [36] 


\subsection{Type-II Quantum Wells on InP Substrate}

In this dissertation, the InGaAs/GaAsSb type-II quantum wells structures are used for SWIR and MWIR detection. A study of the band-offsets of InGaAs and GaAsSb shows that these materials have a type-II band alignment [73-76]. The type-II band alignment of InGaAs/GaAsSb quantum wells is similar to that of InAs/GaSb SLSs, but for detector applications, InGaAs/GaAsSb material systems are usually grown on an InP substrate for photodiodes applications, while InAs/GaSb material systems are grown on $\mathrm{GaSb}$ or InAs substrate. There are a number of advantages in using an InP substrate. These include mature device fabrication, material epi-growth, availability of high-quality 4-inch InP substrates and so on. Since these quantum wells structure can be grown on InP substrate using lattice matched or strain compensated materials, the generation of crystal defects by the lattice mismatch issue can be eliminated. Therefore, lower dark currents can be expected compared to the relaxed InGaAs device.

The difference between type-I and type-II band alignments are shown in Figure 2.6. In a type-I quantum well, the electron and holes are both confined in the same layer. The type-II quantum well can be called as "spatially indirect band gap" semiconductor, since the electrons are confined in one layer, while the holes are confined in a different layer. Here in the InGaAs/GaAsSb type-II quantum wells, electrons are confined in the InGaAs wells, while the holes are confined in the GaAsSb wells. The spatially-indirect type-II transition of InGaAs/GaAsSb quantum wells allows for longer wavelength absorption than that of both InGaAs and GaAsSb bulk materials. 
One disadvantage of the type-II quantum wells is a small electron-hole wave function overlap. Since a larger wave function overlap is desirable to achieve strong absorption, the electron-hole wave function overlap needs to be optimized $[77,78]$.

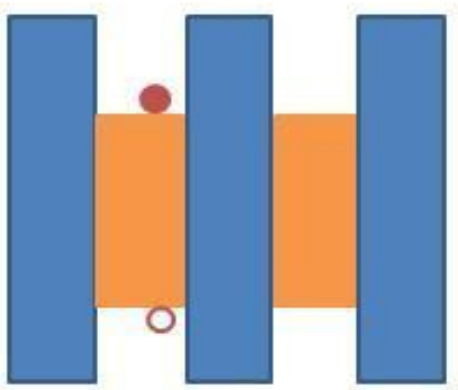

Type-I quantum well

\section{Conduction Band}

Valence Band

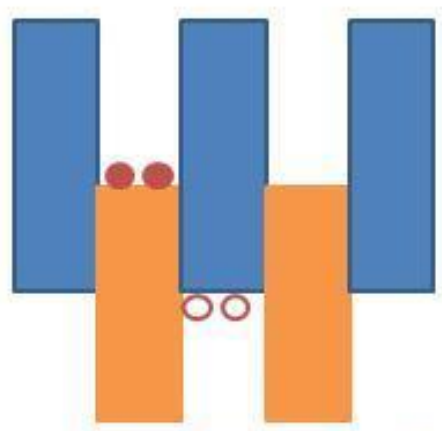

Type-II quantum well

Figure 2.6 Band alignment of type-I and type-II quantum wells

Sidhu et. al. [34] initially demonstrated an InP based PIN photodiode using 150 pairs of lattice matched InGaAs/GaAsSb MQWs with a peak detectivity of $3.8 \times 10^{9}$ $\mathrm{cm} \cdot \mathrm{Hz}^{0.5} \mathrm{~W}^{-1}$ at room temperature at $2.26 \mu \mathrm{m}$. And the peak type-II detectivity at $200 \mathrm{~K}$ is $5.6 \times 10^{10} \mathrm{~cm} \cdot \mathrm{Hz}^{0.5} \mathrm{~W}^{-1}$ at $2.17 \mu \mathrm{m}$. These values are plotted in Figure 2.7 to compare to the detectivity of various infrared detectors. Inada et. al. [79] used 250 pairs of the aforementioned quantum wells as the absorption region to achieve the responsivity $0.6 \mathrm{~A} / \mathrm{W}$ at $2.2 \mu \mathrm{m}$ with dark current density of $0.92 \mathrm{~mA} / \mathrm{cm}^{2}$. The InP based separateabsorption-multiplication (SAM) APDs using lattice matched InGaAs/GaAsSb type-II quantum wells as the absorption region were also reported [80, 81]. 


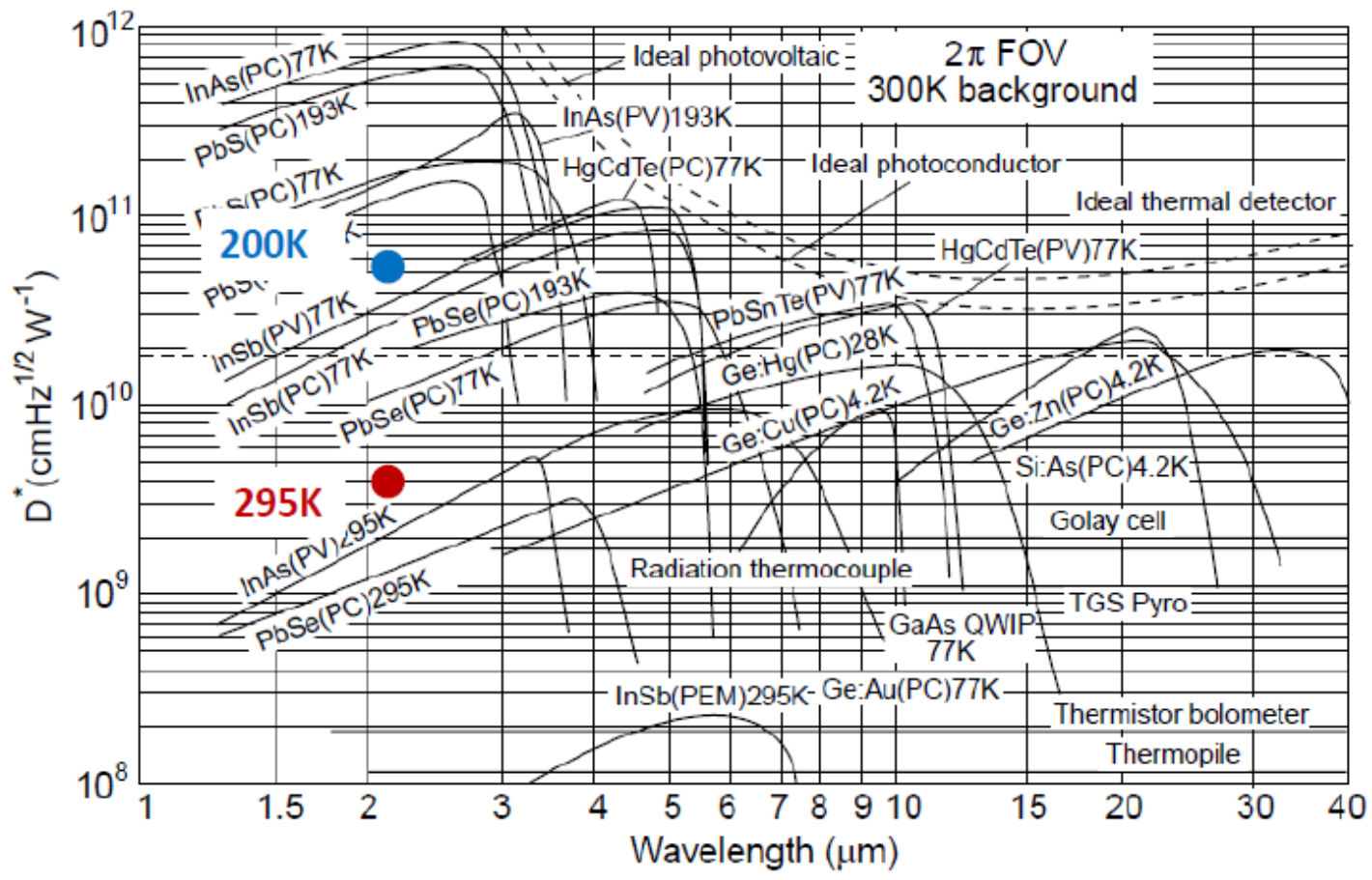

Figure 2.7 Specific detectivity results of Sidhu's initial photodiodes compared to other infrared devices [36]

The goal of this work is to improve the performance of these InP based type-II quantum wells photodiodes, in terms of improving the detectivity performance and extending the detection wavelength. In my research, I have designed and fabricated several new photodiodes with InGaAs/GaAsSb type-II quantum wells as absorption region. These photodiodes show promising results for SWIR and MWIR detection at operating temperature appropriate for thermoelectric or uncooled operation. The design procedure and device characterization results will be presented in the following chapters. 


\section{Chapter 3: Design of InGaAs/GaAsSb Type-II}

\section{Quantum Wells Structures}

\subsection{Introduction}

In order to engineer the optical and electronic properties of InGaAs/GaAsSb type-II quantum wells, the electronic band structure of the quantum wells structure must be known. In this chapter, I use the k.p method, known for calculating the band structure and optical properties of semiconductors, to model these type-II quantum wells.

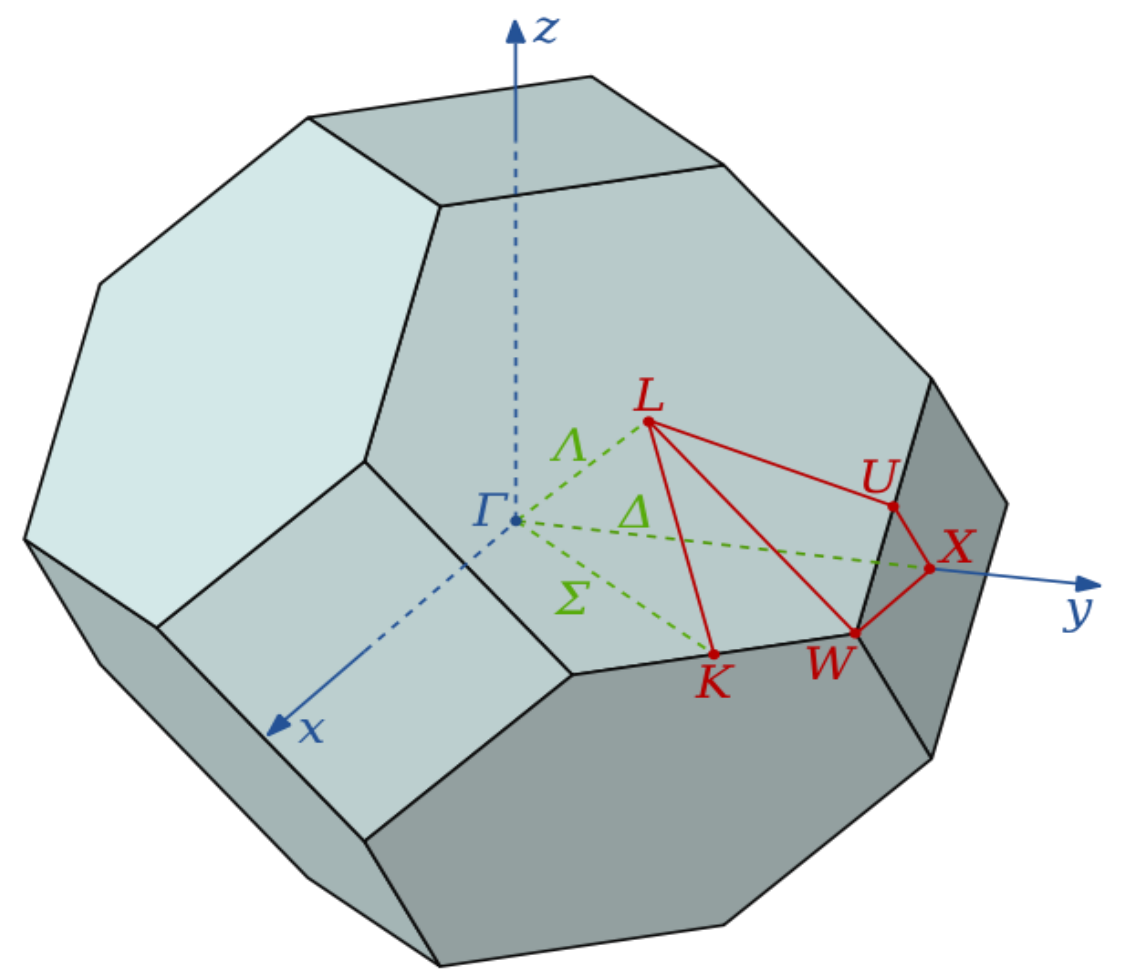

Figure 3.1 First Brillouin zone of FCC lattice showing symmetry labels for high symmetry lines and points. This figure is taken from Reference [82]

In the $\mathrm{k} \cdot \mathrm{p}$ method, the energy states are determined by expanding the wave functions in the vicinity of a high symmetry point of brillouin zone (such as $\Gamma, \mathrm{X}, \mathrm{L}$ points as 
shown in Figure 3.1) through the perturbation method [83]. Therefore, the $\mathrm{k} \cdot \mathrm{p}$ method is only accurate over a small $\mathbf{k}$ range near the local extreme point (such as $\mathbf{k}=0$ for direct band gap semiconductor), but not necessarily accurate for the large $\mathbf{k}$ range. However, knowledge of the E-k relationship over a small $\mathbf{k}$ span is generally sufficient to determine the optical and electronic properties for direct band gap III-V compound semiconductors.

\subsection{The Six-band k•p Method}

To model InGaAs/GaAsSb quantum wells with the $\mathrm{k} \cdot \mathrm{p}$ method, I applied the envelope function approximation to hetero-structures by allowing the material parameters to be functions of space. The detail of the envelope function approximation can be found in reference [83]. In short, differential equations are constructed to describe the electronic states in the quantum wells. For electrons in the conduction band, a single differential equation similar to the Schrödinger equation is constructed. For holes in the valence band, a more complicated system of coupled ordinary differential equations is used including the coupling between heavy holes, light holes and spin-orbit holes. A $6 \times 6$ Hamiltonian in the envelope function space is used to describe the valence band structure, which includes the coupling between two heavy hole bands, two light hole bands and two spin-orbit hole bands (thus the name - six-band k·p method) [84, 85]. Moreover, fourband $k \cdot p$ method [86] and eight-band $k \cdot p$ method $[87,88]$ have also been used to describe the electronic states in hetero-structures, where four-band $\mathrm{k} \cdot \mathrm{p}$ method only includes the coupling of heavy hole and light hole bands, and eight-band k.p method includes all the coupling between electrons, heavy holes, light holes and spin-orbit holes. Theoretically, eight-band $\mathrm{k} \cdot \mathrm{p}$ method is more accurate compared to six-band and four-band $\mathrm{k} \cdot \mathrm{p}$ methods especially for narrow band gap material such as InAs and InSb [89]. However, the eight- 
band $\mathrm{k} \cdot \mathrm{p}$ method can lead to spurious (unphysical) solutions in the envelope function calculation of confines states [90-94]. It is very difficult, sometimes even impossible, to discriminate the real physical states from the spurious states. This problem has become more than a numerical headache for researchers in the community for years. Even though different approaches have been proposed to eliminate the spurious solutions, it is still not effective to completely remove the spurious states and solve this problem [91-93]. Given that the band gap of the materials discussed in the dissertation are relatively large compared with InAs and InSb, I used the six-band $\mathrm{k} \cdot \mathrm{p}$ method to describe the InGaAs/GaAsSb type-II quantum wells.

In the six-band $\mathrm{k} \cdot \mathrm{p}$ method, the conduction band is usually characterized by a parabolic band model

$$
H^{c}(\boldsymbol{k})=\left(\frac{\hbar^{2}}{2 m_{e}}\right)\left(k_{t}^{2}+k_{z}^{2}\right)+V_{e}(z)+a_{c}\left(\varepsilon_{x x}+\varepsilon_{y y}+\varepsilon_{z z}\right)
$$

where $\mathbf{k}$ is wave vector, $\mathrm{k}_{\mathrm{t}}{ }^{2}=\mathrm{k}_{\mathrm{x}}{ }^{2}+\mathrm{k}_{\mathrm{y}}{ }^{2}, \mathrm{~m}_{\mathrm{e}}$ is the electron effective mass, $\mathrm{V}_{\mathrm{e}}(\mathrm{z})$ is the potential energy of the unstrained conduction band edge, $a_{c}$ is the conduction band hydrostatic deformation potential, and $\varepsilon_{\mathrm{xx}}, \varepsilon_{\mathrm{yy}}$, and $\varepsilon_{\mathrm{zz}}$ are the strain of epi-layer grown on the substrate. Here the conduction band non-parabolic effect [95] and anisotropic effect [96] are ignored.

The valence band structure is characterized by the six-band Hamiltonian in the envelope-function space including the coupling of heavy hole (hh), light hole (lh), and spin-orbit split-off (so) bands. Under axial approximation, it has been shown that the $6 \times 6$ Hamiltonian can be transformed into a block-diagonalized Hamiltonian as follows[84, 85]: 


$$
H_{6 \times 6}^{v}(k)=\left(\begin{array}{cc}
H_{3 \times 3}^{U} & 0 \\
0 & H_{3 \times 3}^{L}
\end{array}\right)
$$

Here $\mathrm{H}_{3 \times 3}^{\mathrm{U}}$ and $\mathrm{H}_{3 \times 3}^{\mathrm{L}}$ are represented as below:

$$
H_{3 \times 3}^{\sigma}=-\left(\begin{array}{ccc}
P+Q-V_{h}(z) & R_{k} \pm i S_{k} & \sqrt{2} R_{k} \pm \frac{i}{\sqrt{2}} S_{k} \\
R_{k} \mp i S_{k} & P-Q-V_{h}(z) & \sqrt{2} Q \pm i \sqrt{\left.\frac{3}{2} S_{k}\right)} \\
\sqrt{2} Q \mp i \sqrt{\frac{3}{2} S_{k}} & \sqrt{2} R_{k} \mp \frac{i}{\sqrt{2}} S_{k} & P+\Delta-V_{h}(z)
\end{array}\right)
$$

And $\sigma=U, L$

where

$$
\begin{gathered}
P=P_{k}+P_{\varepsilon} \\
Q=Q_{k}+Q_{\varepsilon} \\
P_{k}=\left(\frac{\hbar^{2}}{2 m}\right) \gamma_{1}\left(k_{t}^{2}+k_{z}^{2}\right) \\
Q_{k}=\left(\frac{\hbar^{2}}{2 m}\right) \gamma_{2}\left(k_{t}^{2}-2 k_{z}^{2}\right) \\
S_{k}=\left(\frac{\hbar^{2}}{2 m}\right) \sqrt{3}\left(\frac{\gamma_{2}+\gamma_{3}}{2}\right) k_{t}^{2} \\
R_{k}=\left(\frac{\hbar^{2}}{2 m}\right) 2 \sqrt{3} \gamma_{3} k_{t} k_{z} \\
P_{\varepsilon}=-a_{v}\left(\varepsilon_{x x}+\varepsilon_{y y}+\varepsilon_{z z}\right) \\
Q_{\varepsilon}=-\frac{b}{2}\left(\varepsilon_{x x}+\varepsilon_{y y}-2 \varepsilon_{z z}\right)
\end{gathered}
$$

and where $\mathrm{V}_{\mathrm{h}}(\mathrm{z})$ is the unstrained valence band edge; $\gamma_{1}, \gamma_{2}$, and $\gamma_{3}$ are the Luttinger parameters; $\mathrm{a}_{\mathrm{v}}$ and $\mathrm{b}$ are the Bir-Pikus deformation potentials; and $\boldsymbol{\Delta}(\mathrm{z})$ is the spin-orbit split-off energy, and $\varepsilon_{\mathrm{xx}}, \varepsilon_{\mathrm{yy}}$, and $\varepsilon_{\mathrm{zz}}$ are the strain of epi-layer grown on the substrate. 
For a quantum well structure, the electron wave function can be defined as

$$
\Psi_{\mathrm{n}, \mathrm{k}_{\mathrm{t}}}^{\mathrm{c \eta}}=\frac{e^{i \mathrm{k}_{\mathrm{t}} \rho}}{\sqrt{A}} \phi_{n}\left(z ; \mathrm{k}_{\mathrm{t}}\right)|S, \eta\rangle
$$

where $\mathrm{A}$ is the area of the quantum well, $\mathrm{k}_{\mathrm{t}}$ is the real transverse wave number, $\rho=$ $\sqrt{\mathrm{x}^{2}+\mathrm{y}^{2}}, \phi_{\mathrm{n}}$ is the envelop function of the $n^{\text {th }}$ conduction subband, $\eta$ is the electron spin state, and the basis $|S, \eta\rangle$ is Block wave function for conduction band. The envelop function should be normalized to one. The conduction band energy $E_{n}^{c}\left(k_{t}\right)$ can be determined by solving the eigenvalues and eigenvectors of the equation (3.6) as below for different $\mathrm{k}_{\mathrm{t}}$ values.

$$
H^{c} \phi_{n}\left(z ; \mathrm{k}_{\mathrm{t}}\right)=E_{n}^{c}\left(k_{t}\right) \phi_{n}\left(z ; \mathrm{k}_{\mathrm{t}}\right)
$$

Similarly, the hole wave function can be written as

$$
\Psi_{\mathrm{m}, \mathrm{k}_{\mathrm{t}}}^{\mathrm{v} \sigma}=\frac{e^{i \mathrm{k}_{\mathrm{t}} \rho}}{\sqrt{A}} \sum_{i=h h, l h, s o} g_{m, i}^{\sigma}\left(z ; \mathrm{k}_{\mathrm{t}}\right) \mid \mu_{i}^{\sigma}>
$$

where $\mathrm{g}_{\mathrm{m}, \mathrm{i}}^{\sigma}$ is the envelop function of the $m^{\text {th }}$ valence subband. Similarly, the valence subband energy dispersion can be determined by solving equation (3.8):

$$
\sum_{i=h h, l h, s o} H_{3 \times 3, i j}^{\sigma} g_{m, j}^{\sigma}=E_{\sigma, m}^{v}\left(k_{t}\right) g_{m, i}^{\sigma}\left(z ; \mathrm{k}_{\mathrm{t}}\right)
$$

where $\mathrm{i}=\mathrm{hh}$, lh and so for the heavy hole, light hole, and split-off bands. In both equation (3.6) and (3.8), the wave number $\mathrm{k}_{\mathrm{z}}$ should interpreted as a differential operator $-\mathrm{i} \frac{\partial}{\partial \mathrm{z}}$. In this dissertation, I solve these equations using the finite difference method. All of the differential operators can be written as $\mathrm{A}(\mathrm{z}) \frac{\partial^{2}}{\partial \mathrm{z}^{2}}$ and $\mathrm{B}(\mathrm{z}) \frac{\partial}{\partial \mathrm{z}}$, where A and B stand for the position dependent parameters. In order to guarantee the Hermitian properties of the Hamiltonian (equation 3.1 and 3.3), the following finite difference formulas were used while solving equation (3.6) and (3.8) [84]. 


$$
\begin{aligned}
\left.A(z) \frac{\partial^{2} \mathrm{~g}}{\partial \mathrm{z}^{2}}\right|_{\mathrm{z}=\mathrm{z}_{\mathrm{i}}} \rightarrow & \left.\frac{\partial}{\partial \mathrm{z}}\left(A(\mathrm{z}) \frac{\partial \mathrm{g}}{\partial \mathrm{z}}\right)\right|_{\mathrm{z}=\mathrm{z}_{\mathrm{i}}} \\
\approx & \frac{A\left(z_{i+1}\right)+A\left(z_{i}\right)}{2(\Delta \mathrm{z})^{2}} \mathrm{~g}\left(\mathrm{z}_{\mathrm{i}+1}\right) \\
& -\frac{A\left(z_{i+1}\right)+2 A\left(z_{i}\right)+A\left(z_{i-1}\right)}{2(\Delta \mathrm{z})^{2}} \mathrm{~g}\left(\mathrm{z}_{\mathrm{i}}\right) \\
& +\frac{A\left(z_{i-1}\right)+A\left(z_{i}\right)}{2(\Delta \mathrm{z})^{2}} \mathrm{~g}\left(\mathrm{z}_{\mathrm{i}-1}\right) \\
& \left.\left.\mathrm{B}(\mathbf{z}) \frac{\partial \mathrm{g}}{\partial \mathrm{z}}\right|_{\mathrm{z}=\mathrm{z}_{\mathrm{i}}} \rightarrow \frac{1}{2}\left(\mathrm{~B}(\mathrm{z}) \frac{\partial \mathrm{g}}{\partial \mathrm{z}}+\frac{\partial(\mathrm{Bg})}{\partial \mathrm{z}}\right)\right|_{\mathrm{z}=\mathrm{z}_{\mathrm{i}}} \\
\approx & \frac{B\left(z_{i+1}\right)+B\left(z_{i}\right)}{4 \Delta \mathrm{z}} \mathrm{g}\left(\mathrm{z}_{\mathrm{i}+1}\right)-\frac{B\left(z_{i-1}\right)+B\left(z_{i}\right)}{4 \Delta \mathrm{z}} \mathrm{g}\left(\mathrm{z}_{\mathrm{i}-1}\right)
\end{aligned}
$$

where $\Delta \mathrm{z}=\mathrm{z}_{\mathrm{i}+1}-\mathrm{z}_{\mathrm{i}}=\mathrm{z}_{\mathrm{i}}-\mathrm{z}_{\mathrm{i}-1}$.

\subsection{Design of InGaAs/GaAsSb Type-II Quantum Wells}

As discussed in the last chapter, the InGaAs/GaAsSb type-II quantum well structure has electrons are confined in the conduction band of the InGaAs layer, while holes are confined in the valence band of GaAsSb layer. This type-II band line-up has a narrower effective band gap than type-I transitions and can enable longer wavelength detection. Because the electrons and holes are separately confined in adjoining layers, the wave function overlap is smaller than in bulk or type-I quantum well structures. A reduced wave function overlap reduces the light absorption, and the quantum efficiency of photodiodes. Thus, given the same effective band gap, optimal performance requires a wave function overlap as high as possible. 


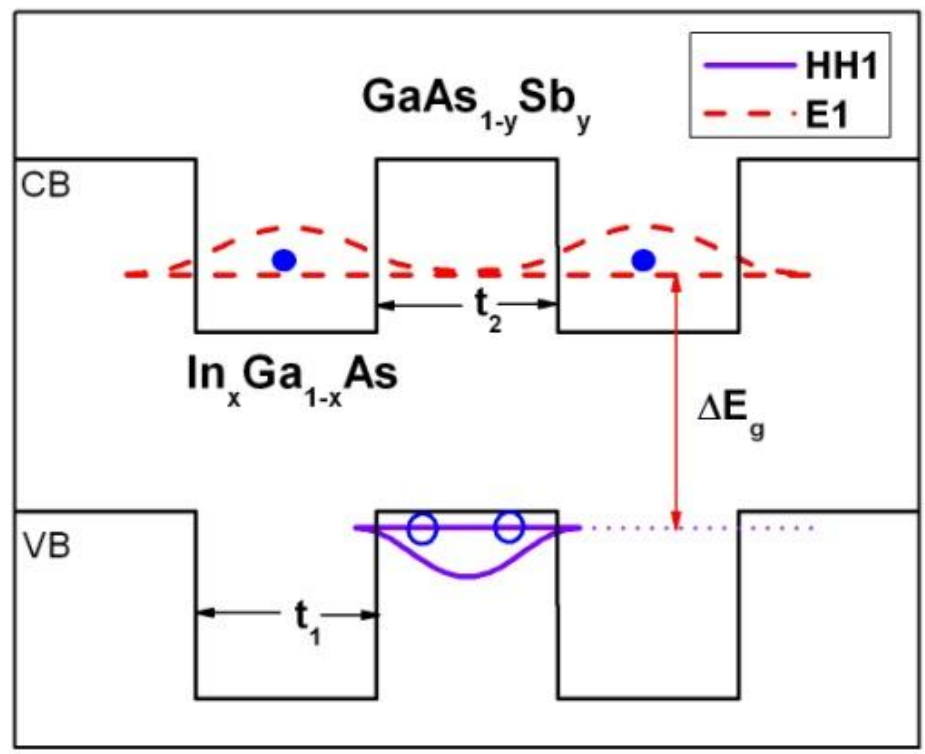

Figure 3.2 Schematic diagrams for the band alignment and the wave function components for the InGaAs/GaAsSb type-II quantum well

Figure 3.2 shows a schematic of the type-II InGaAs/GaAsSb quantum wells. I used equations (3.6) and (3.8) to calculate the energy level and wave function of the subbands in type-II quantum wells. The parameters used to describe the quantum well properties such as the band gaps, conduction band offsets, and elastic constants are taken from recent theoretical and experimental results [53, 97].

Figure 3.3 plots the subbands of the discrete energy level $\mathrm{E}$ as function of $\mathrm{k}_{\mathrm{i}}$ for $5 \mathrm{~nm}$ InGaAs/5nm GaAsSb lattice matched quantum well. I define the energy difference between the ground state of electron state in quantum well and the ground state of heavy hole state to be effective band gap of the type-II quantum well. We can see that the effective band gap in this case is around $0.5 \mathrm{eV}$, which is smaller than the band gap of 
both InGaAs and GaAsSb materials (which are around $0.72 \mathrm{eV}$ and $0.73 \mathrm{eV}$ respectively [53]).

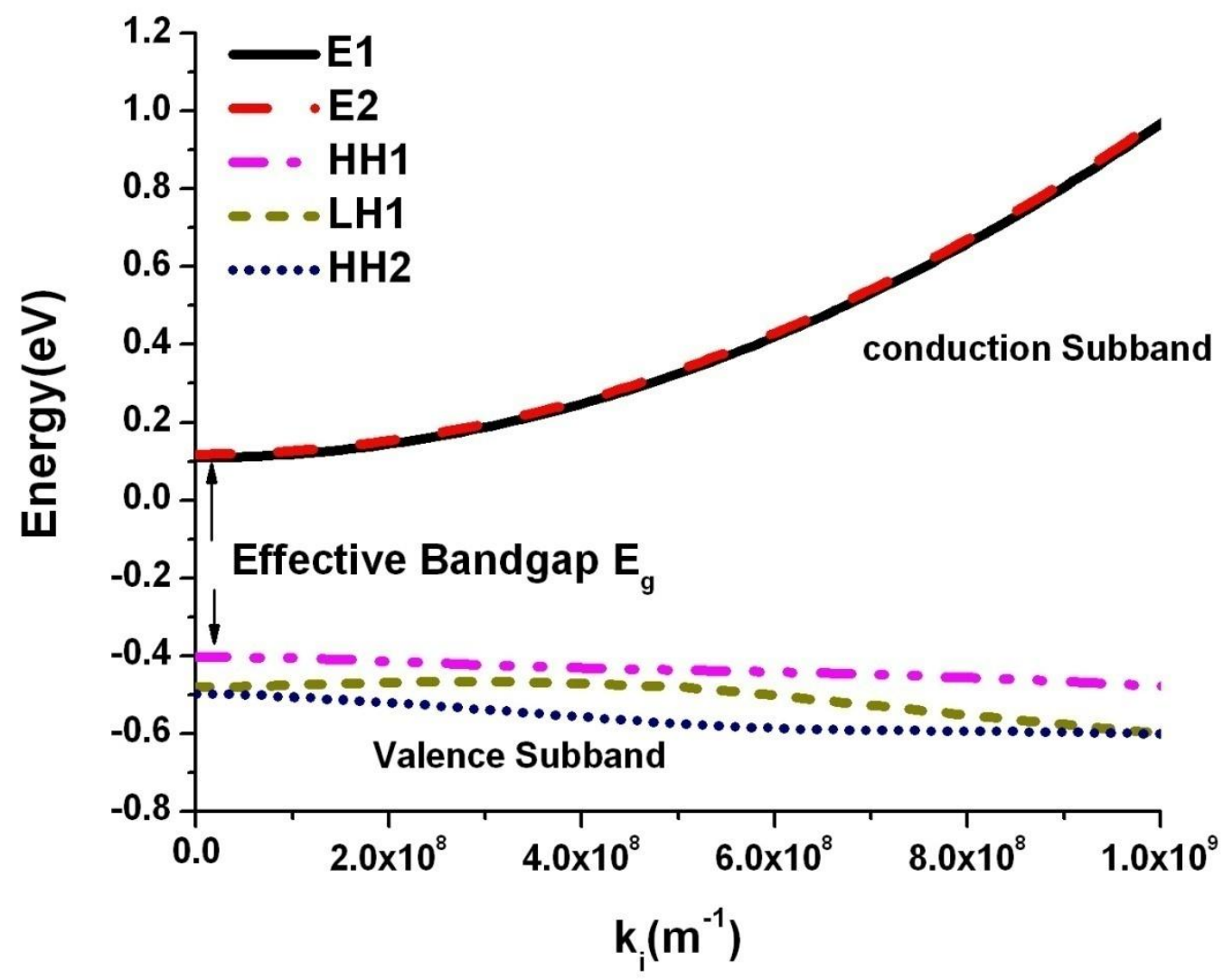

Figure 3.3 Subband energy for the $5 \mathrm{~nm}$ InGaAs/5nm GaAsSb lattice matched type-II quantum well on InP substrate

While photodiodes absorption regions will have a large number of quantum wells, our modeling shows that the simplified structure in Figure 3.2 gives results similar to a structure with more periods. Thus this simplified structure was used for in all design calculations. In order to optimize transition wavelength and wave function overlap, the thickness of InGaAs $\left(t_{1}\right)$, thickness of GaAsSb $\left(t_{2}\right)$, indium (In) content in $\operatorname{In}_{x} G_{1-x} A s$ and 
antimony $(\mathrm{Sb})$ content in $\mathrm{GaAs}_{1-\mathrm{y}} \mathrm{Sb}_{\mathrm{y}}$ can be adjusted, indicating to there are four degrees of freedom in the this quantum wells system.

\subsubsection{Effects of the Thickness of the Quantum Well Layers}

One clear way to increase the detection wavelength is to make the layers in the quantum wells structure thicker. The effects of thickness changes for lattice matched InGaAs and GaAsSb are shown in Figure 3.4(a) and 3.4(b).

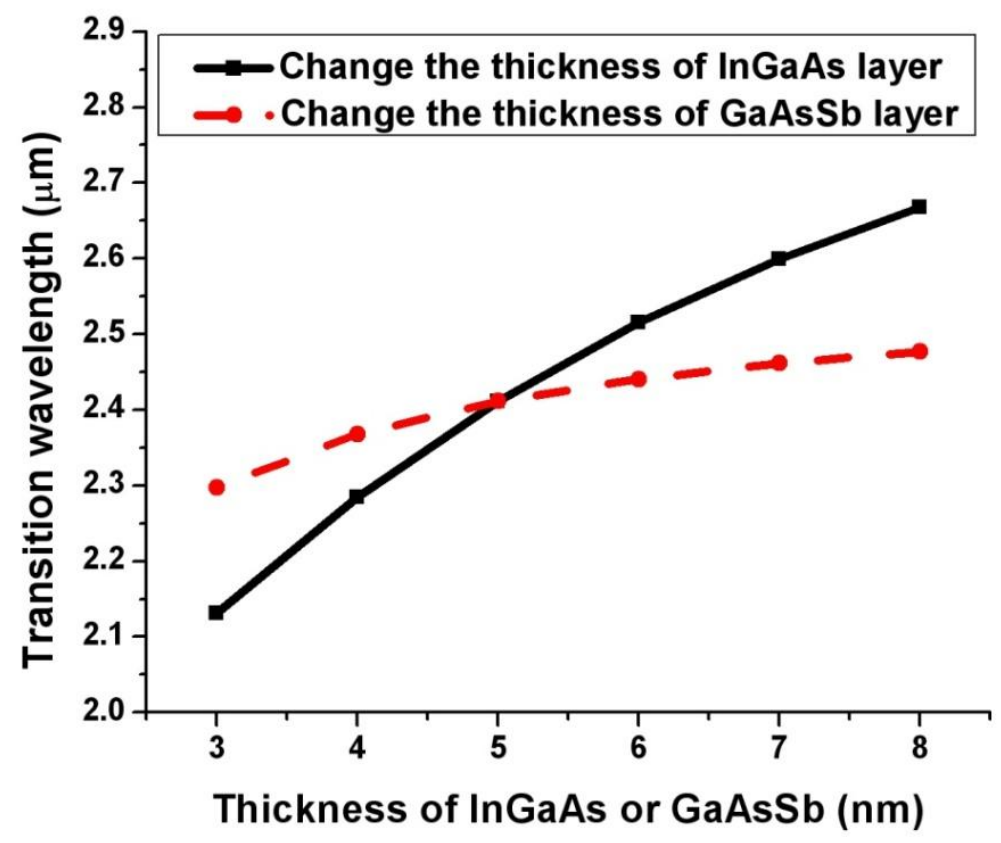

(a) 


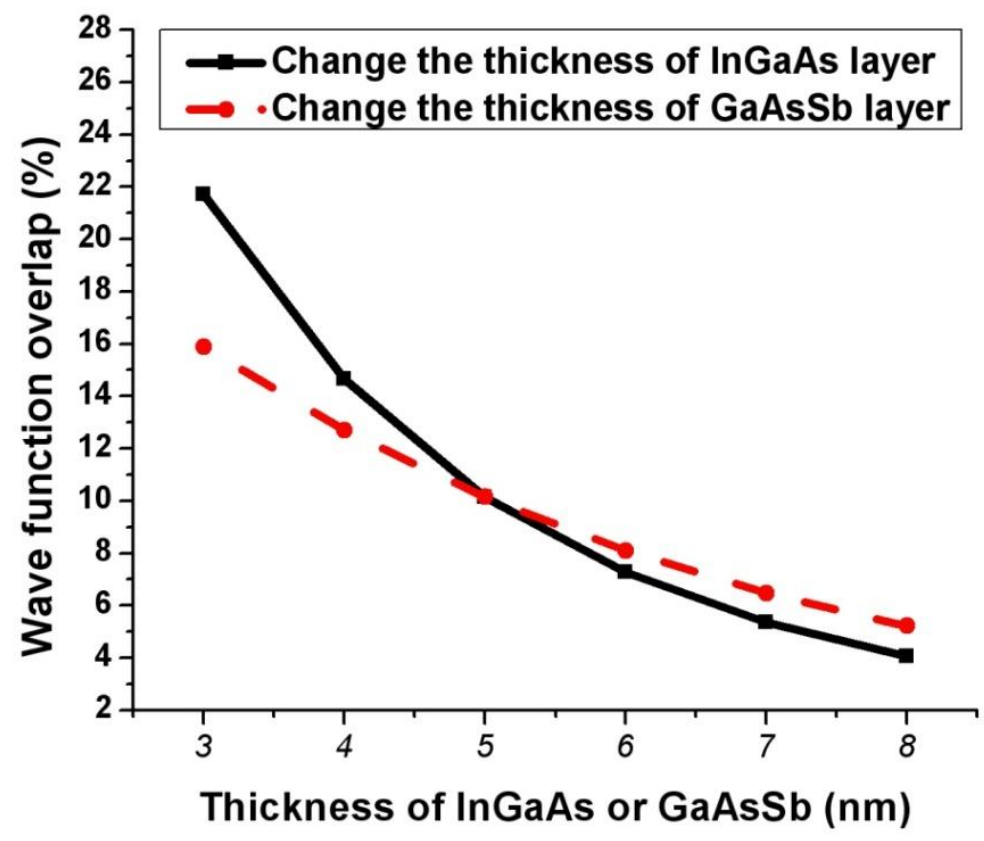

(b)

Figure 3.4 (a) Transition wavelength and (b) wave-function overlap dependence on the thickness change of lattice-matched InGaAs or GaAsSb. The simulation was carried out by changing the layer thickness of one material while keeping the layer thickness of the other at $5 \mathrm{~nm}$.

The trade-off between wavelength and wave function overlap is evident. Increases in the detection wavelength with thickness changes leads to a reduction in the wave function overlap. To further illustrate this point, Figure 3.5 plots the wave function overlap as a function of the detection wavelength. As shown in the figure, it is more effective to increase the thickness of InGaAs to get the longer transition wavelength, since it can achieve a larger increase of transition wavelength when sacrificing the same amount of wave function overlap. Similarly, in order to achieve a larger wave function overlap, it is more effective to decrease the thickness of GaAsSb. Therefore, the thickness of InGaAs layer should be larger than the thickness of GaAsSb to optimize the transition wavelength and wave function overlap. The physical reason of these relationships is that the effective 
mass of the heavy hole confined in GaAsSb layer is much larger than that of electron confined in InGaAs layer. Therefore a reduction in the thickness of GaAsSb increases the wave function overlap due to increased electron penetration into the barrier but with a small decrease in the detection wavelength.

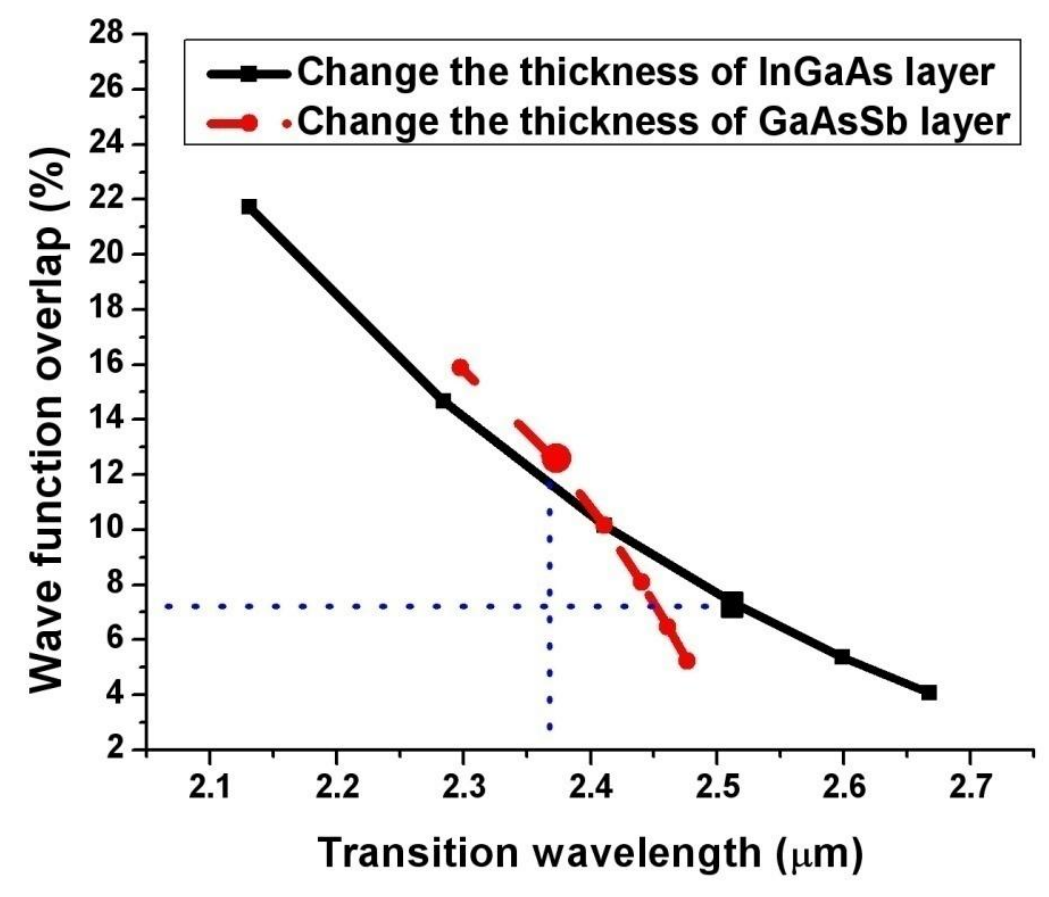

Figure 3.5 Wave function overlap versus transition wavelength by changing the layer thickness of one material while keeping the layer thickness of the other material fixed at $5 \mathrm{~nm}$

\subsubsection{Effects of the Composition of the Quantum Well Layers}

In addition to changing the thickness of the layers, compositional changes can be used to increase the detection wavelength. This requires strain to be added into the system. The band-edge effects due to the strain are illustrated in Figure 3.6. The hydrostatic term $\left(\varepsilon_{\mathrm{xx}}+\varepsilon_{\mathrm{yy}}+\varepsilon_{\mathrm{zz}}\right)$, which is proportional to the first order change in cubic volume due to the strain, will shift the conduction and valence band edges. The shear term $\left(\varepsilon_{\mathrm{xx}}+\right.$ 
$\left.\varepsilon_{\mathrm{yy}}-2 \varepsilon_{\mathrm{zz}}\right)$, which is proportional to the difference between the vertical and in-plane lattice constants due to the strain, will split the heavy hole and light hole states.

a) Compressive strain $a(x)>a_{0}$ $E_{c}$
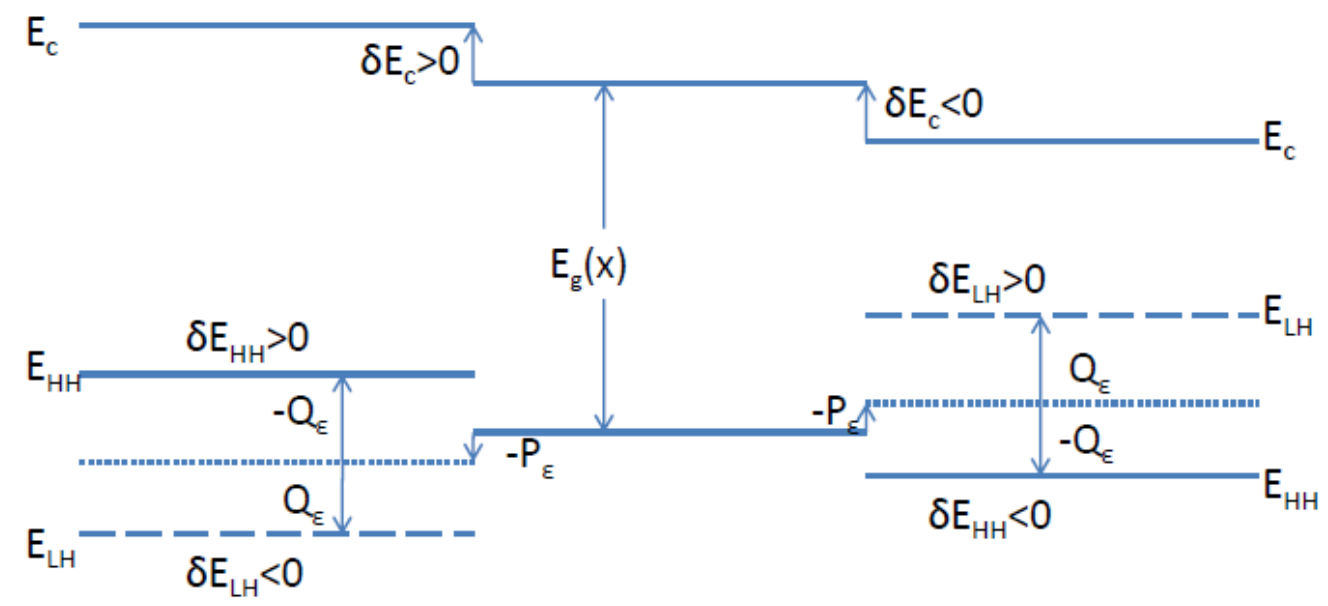

Figure 3.6 Band edges changes for III-V semiconductor under compressive strain, no strain, and tensile strain

To investigate how transition wavelength changes in relation to the composition of quantum wells, simulations that change the composition were carried out. Figure 3.7(a) and 3.7(b) show that the transition wavelength increases as In composition in InGaAs layer or Sb composition in GaAsSb layer increases. 


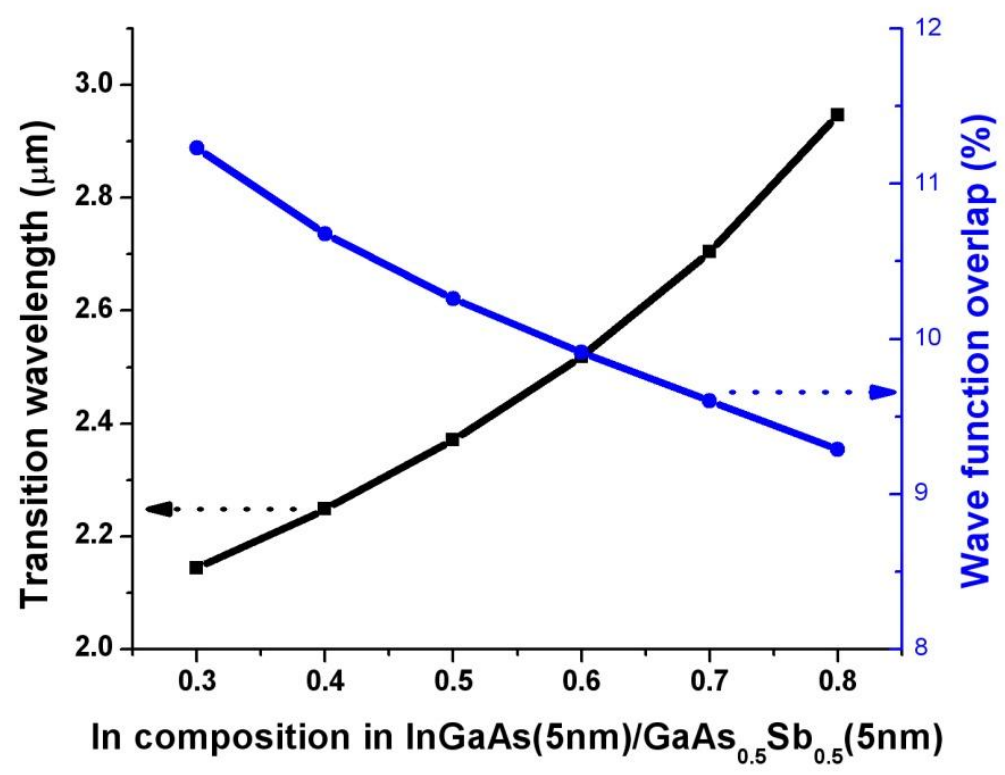

(a)

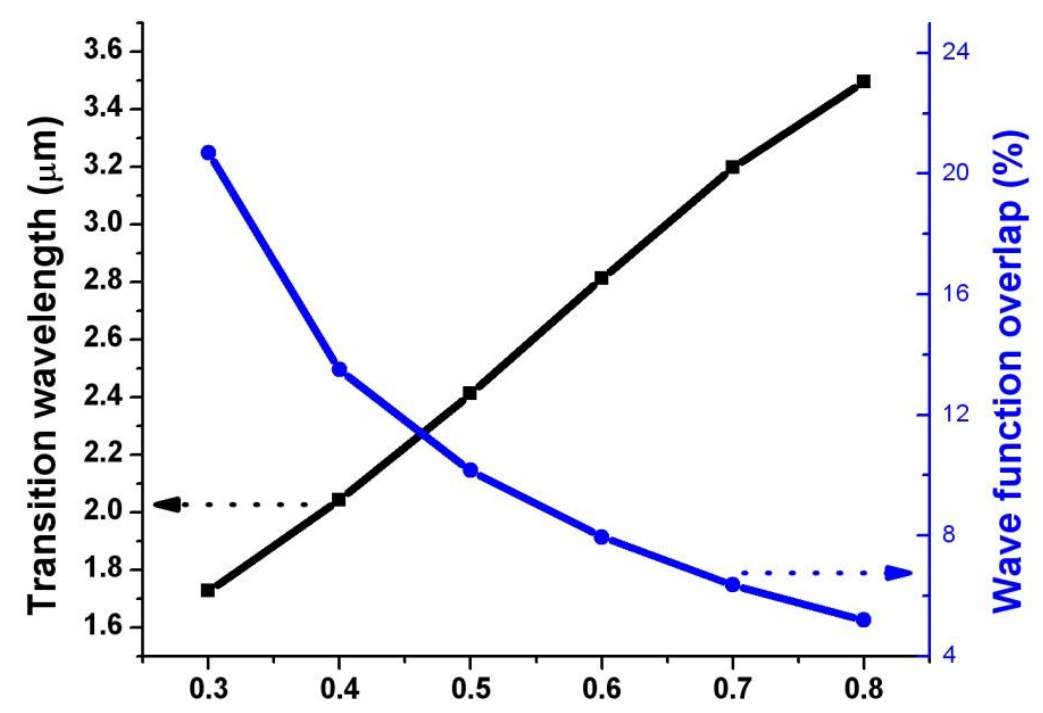

$\mathrm{Sb}$ composition in $\ln _{0.53} \mathrm{Ga}_{0.47} \mathrm{As}(5 \mathrm{~nm}) / \mathrm{GaAsSb}(5 \mathrm{~nm})$

(b)

Figure 3.7 The dependence of the transition wavelength and the wave-function overlap versus (a) the In composition change while keeping GaAsSb lattice-matched to InP and (b) the Sb composition change while keeping InGaAs lattice-matched to InP 
How the band edge changes as In composition in InGaAs layer increases or $\mathrm{Sb}$ composition in GaAsSb layer increases is roughly shown in Figure 3.8.

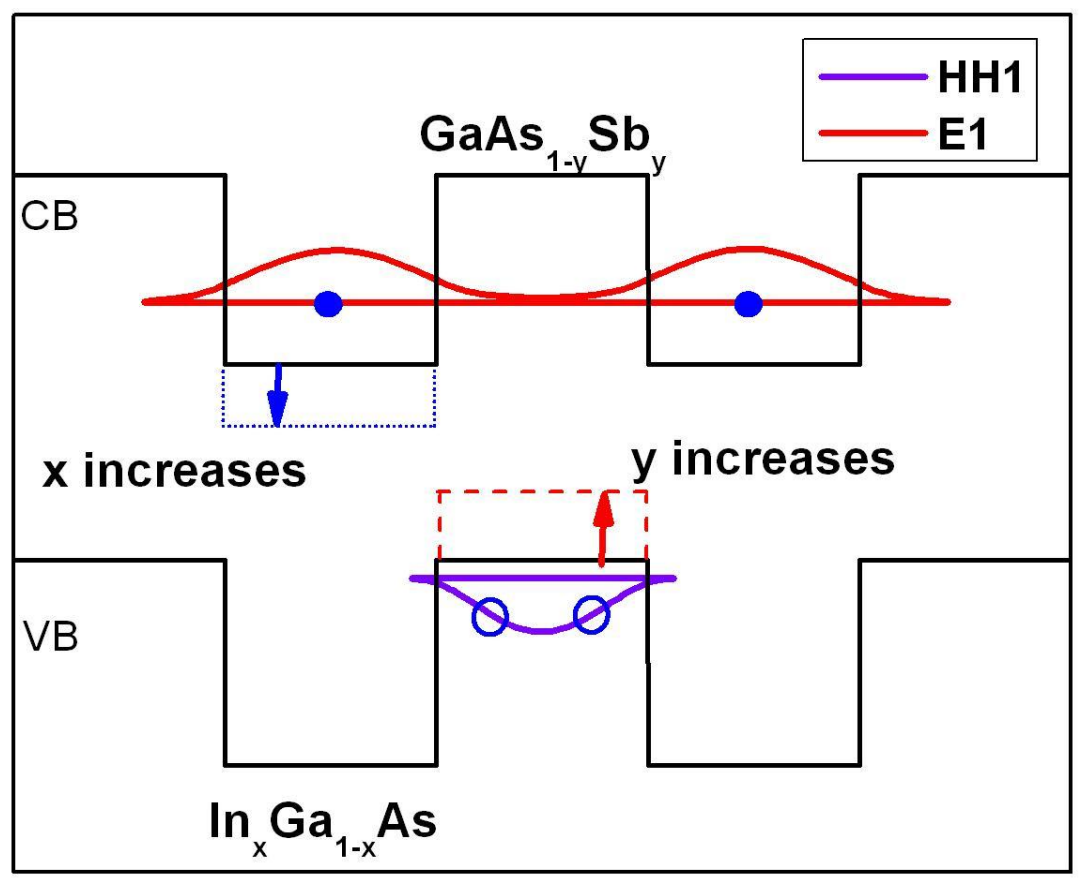

Figure 3.8 Schematic diagram of the band alignment for a InGaAs/GaAsSb type-II QW about how the CB of InGaAs and HH of GaAsSb move with increase of In composition (x) in InGaAs and Sb composition (y) in GaAsSb

In Figure 3.8, we can see that the conduction edge decreases as In composition increases, and the valence edge increases as Sb composition increase. Both trends extend the transition wavelength between electron and hole. Moreover, the wave function overlap shows the opposite trend as the In or Sb composition increases, since the wells become deeper. These results also indicate that it is more effective to add compressive strain in GaAsSb layer in order to achieve longer transition wavelength. For the longest detection wavelength, compressive strain should be added to both layers. While this approach may work for thin active regions such as the semiconductor lasers, detectors 
require thick absorption regions for adequate performance. As a result, compressive strain can't be used in both materials if we simply repeat using those two layers to get a thick absorption region.

\subsubsection{Strain Compensated InGaAs/GaAsSb MQWs}

The strained quantum wells offer a number of advantages over quantum wells composed of a lattice matched material combination, such as extending absorption wavelength as discussed above. However, when attempting to grow a stack of strained quantum wells, each strained layer will add to the overall stress. Beyond some thickness, termed the critical thickness, the total strain will lead to strain relaxation of the strained quantum wells.

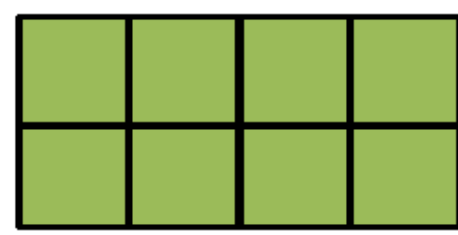

$$
\mathrm{a}>\mathrm{a}_{\mathrm{s}}
$$

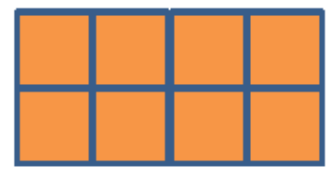

$$
a<a_{s}
$$
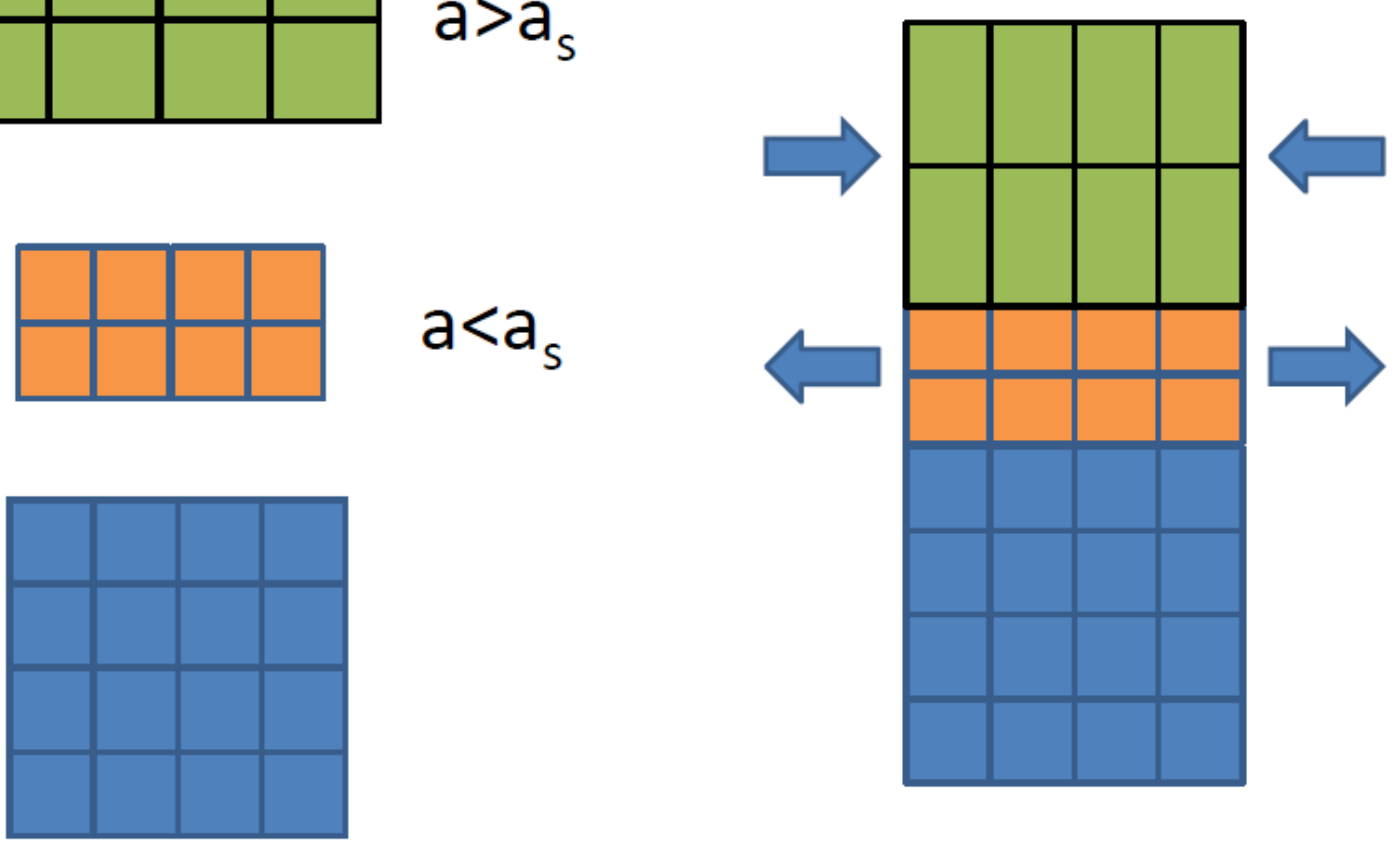

\section{Substrate $a_{s}$}

Figure 3.9 Strain-balanced multi-quantum wells consist of compressive-strained layer and tensilestrained layer 
In order to ensure pseudomorphic growth of multiple layers of quantum wells, a technique called "strain compensation" is commonly used [68, 69, 98-100]. The idea of strain compensation is balancing the strain in one compressive-strained layer by another tensile-strained layer as shown in Figure 3.9. This technique minimizes the total elastic energy of epitaxial layer in order to achieve the stable strain state, which prevents the generation of dislocation defects. As a result, the critical thickness can be extended to thicknesses appropriate for the absorption regions of photodiodes.

The strain compensation condition for a cubic-based multilayer system grown along the $(001)$ axis is $[101,102]$

$$
\begin{aligned}
& \sum_{i=1}^{n} \frac{A_{i} \varepsilon_{i} t_{i}}{a_{i}}=0 \\
& A_{i}=C_{11}^{i}+C_{12}^{i}-2 \frac{\left(C_{12}^{i}\right)^{2}}{C_{11}^{i}}
\end{aligned}
$$

where $\mathrm{t}_{\mathrm{i}}$ is the thickness of the $i^{\text {th }}$ layer, $C_{11}^{i}$ and $C_{12}^{i}$ are elastic constants of the $i^{\text {th }}$ layer, $\mathrm{a}_{\mathrm{i}}$ is the lattice constant of the $i^{\text {th }}$ layer, and $\varepsilon_{\mathrm{i}}$ is the strain of the $i^{\text {th }}$ epi-layer. Experimentalists often use the following equations of strain compensation condition by evaluating the weighted average strain over the layer thickness or including an elastic parameter A.

$$
\begin{gathered}
\sum_{i=1}^{n} \varepsilon_{i} t_{i}=0 \\
\sum_{i=1}^{n} A_{i} \varepsilon_{i} t_{i}=0
\end{gathered}
$$


Theoretically speaking, the equation (3.11) should be more accurate than equation (3.13) and (3.14), since the elastic constants and lattice constant may vary from layer to layer depending the on the material in the layer.

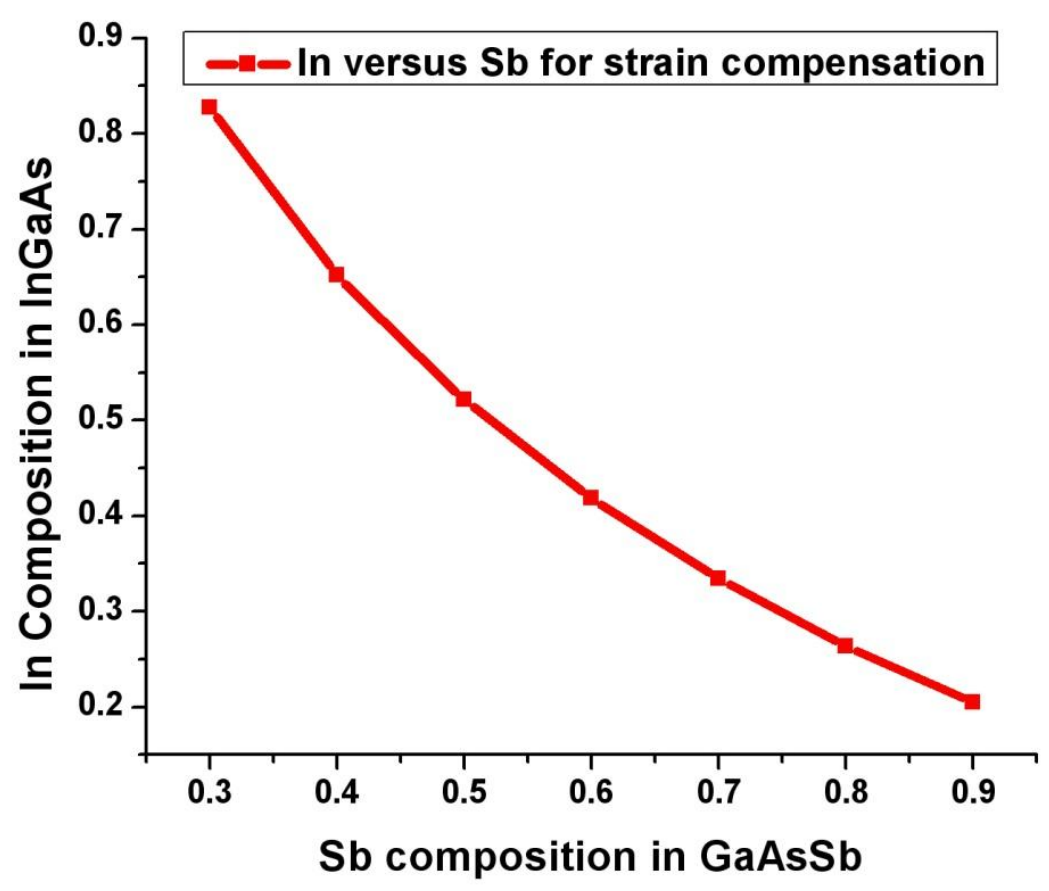

Figure 3.10 Under the strain compensation condition, the In composition in InGaAs versus Sb composition in GaAsSb with layer thickness of $5 \mathrm{~nm}$ for both InGaAs and GaAsSb layers

Using equation (3.11), the relation between In composition in InGaAs layer and $\mathrm{Sb}$ composition in GaAsSb layer can be solved numerically. To maintain strain compensation, the indium composition in InGaAs has to decrease when the antimony composition in GaAsSb increases as shown in Figure 3.10. Figures 3.11 shows the effects of strain compensation on the transition wavelength and wave function overlap. Based on Figure 3.11, higher Sb content in GaAsSb (compressive strain) can achieve a longer detection wavelength up to a mole fraction of 0.8 . After this point, the transition 
wavelength decreases due to the further decrease of the indium composition in the InGaAs layers.

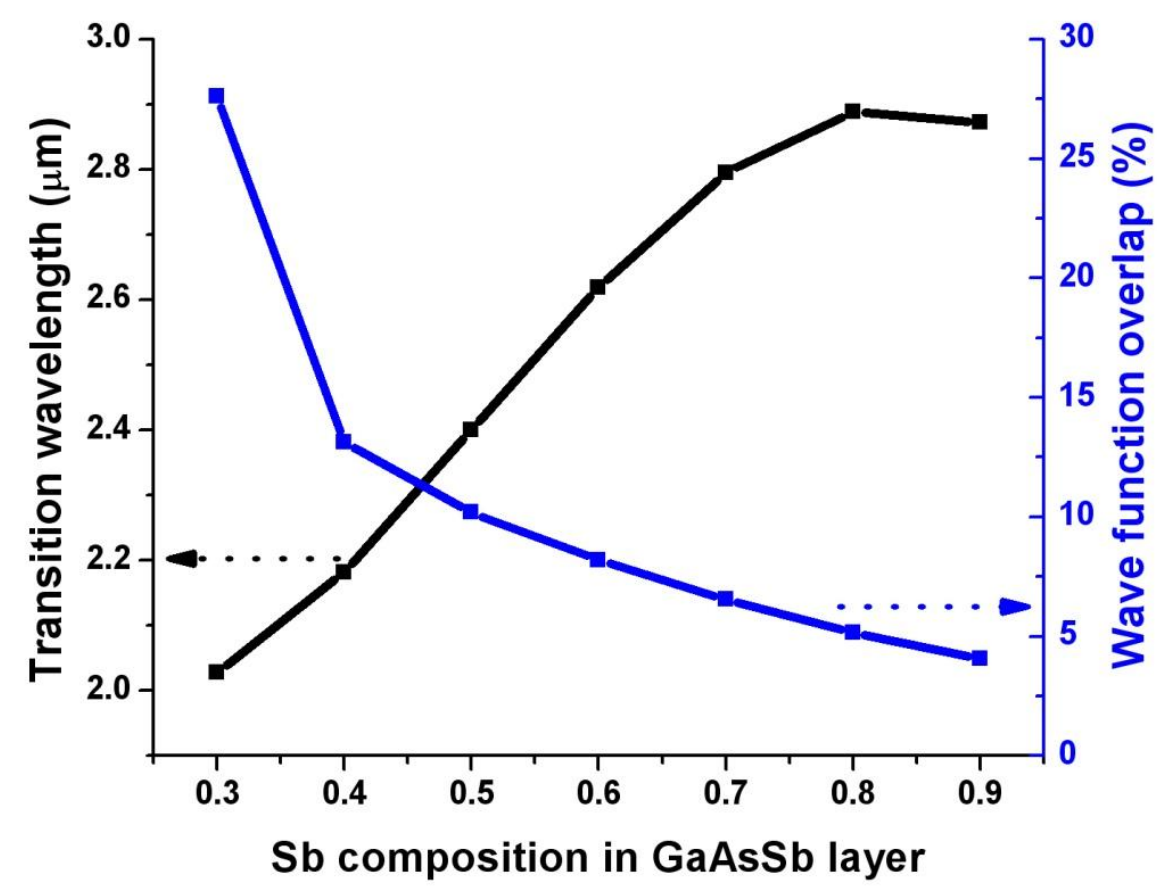

Figure 3.11 Under the strain compensation condition, the transition wavelength and the wavefunction overlap versus Sb composition change with layer thickness of $5 \mathrm{~nm}$ for both $\mathrm{InGaAs}$ and GaAsSb layers

To investigate the maximized transition wavelength in strain compensated quantum well structures, several thickness and composition combinations are tested in simulation. Figure 3.12 plots the transition wavelength as function of wave function overlap for different thicknesses and compositions. It can be concluded that the longest transition wavelength of $4.15 \mu \mathrm{m}$ can be achieved in this type-II quantum well structure but with a small wave function overlap. 


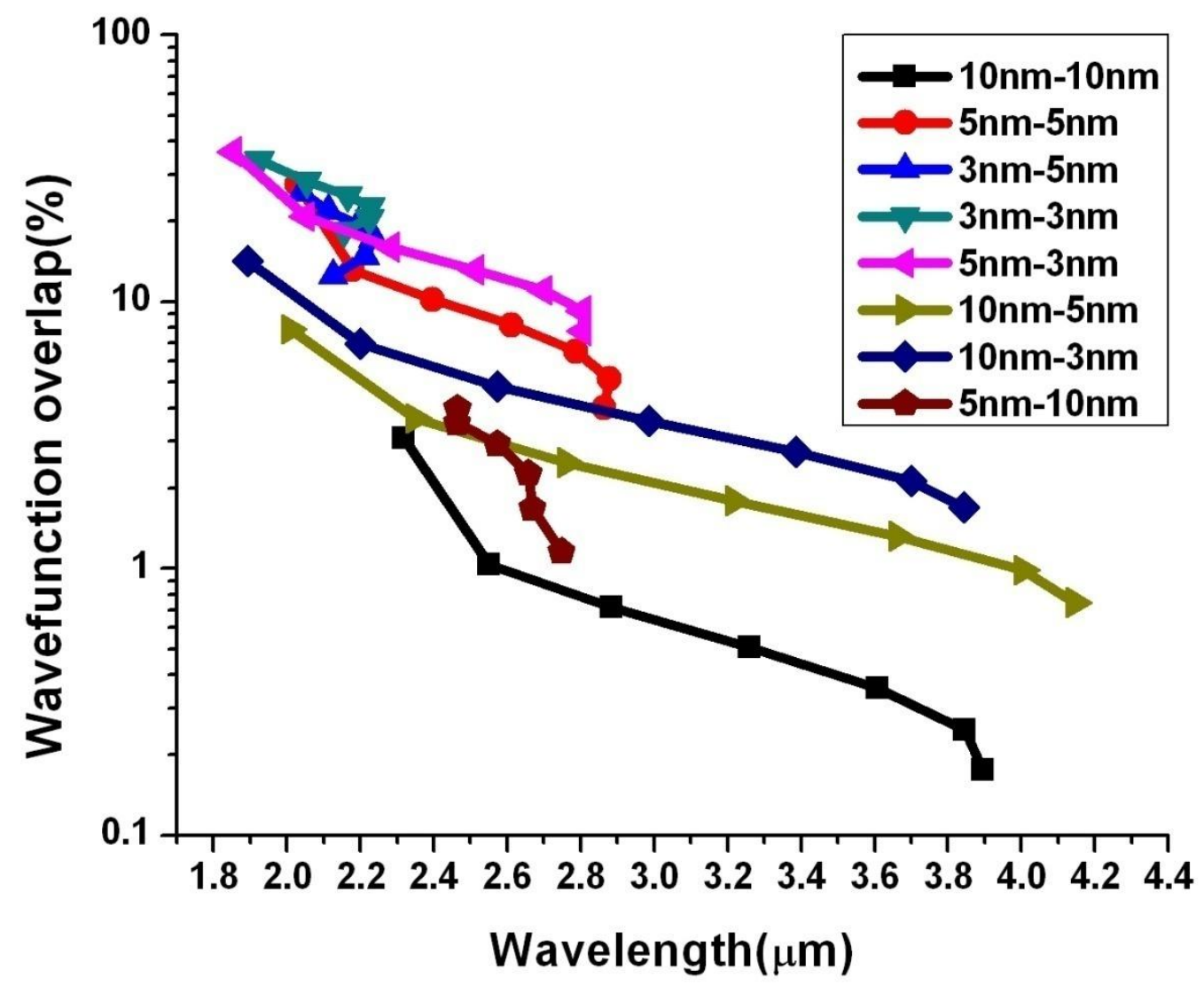

Figure 3.12 Under the strain compensation condition, wave function overlap versus transition wavelength for different thickness combinations for both InGaAs and GaAsSb layers with Sb composition changing from 0.3 to 0.9

\subsection{Limitations of the k•p Method}

The k.p method can give accurate results, in terms of the cut-off wavelength calculation for type-II quantum wells photodiodes. However, the absorption coefficient value based on the six-band $\mathrm{k} \cdot \mathrm{p}$ method for type-II quantum wells structure does not match well with experimental data especially at short wavelength range. The reasons are discussed below. 
First, the $\mathrm{k} \cdot \mathrm{p}$ method is only accurate over a small $\mathbf{k}$ range near the local extreme point. Since the model determines the energy bands by expanding the wave functions in the vicinity of a high symmetry point $(\Gamma, \mathrm{X}, \mathrm{L}$ points) through the perturbation method, the energy bands at larger $\mathbf{k}$ value may not be accurate. Second, for the model used here to calculate the absorption coefficient of the type-II quantum wells, the exciton effect is not included [103].

However, in terms of the design of the quantum wells, the k.p method is still sufficient enough to give the accurate trend while changing the quantum well composition or thickness, which will help to design the quantum well structures.

\subsection{Summary}

In this chapter, six-band $\mathrm{k} \cdot \mathrm{p}$ theory is introduced and used to calculate the transition wavelength and wave function overlap of InGaAs/GaAsSb type-II quantum wells structure. The simulation indicates that in order to maximize the transition wavelength and wave function overlap under strain compensated condition, the thickness of InGaAs layer should be larger than that of GaAsSb layer, and GaAsSb layer should compressively strained, while InGaAs layer is tensile strained. Lastly, the potentially longest absorption wavelength that the InGaAs/GaAsSb strain compensated type-II quantum wells can achieve was explored theoretically. It is found that these InGaAs/GaAsSb strain compensated type-II quantum wells have the potential for absorption across the $2-4 \mu \mathrm{m}$ spectral band. In Chapter 5 , photodiodes using these type-II quantum wells based on the design criteria discussed in this chapter will be studied experimentally. 


\section{Chapter 4: Photodiode Characterization}

Prior to discussing the performance of the InP based InGaAs/GaAsSb type-II quantum well infrared photodiodes, I will discuss the parameters I have used to quantify their performance: dark current, responsivity, dark noise and specific detectivity.

\subsection{PIN Photodiodes}

One of the most common photodiode structures is the PIN photodiode. The schematic of a typical PIN photodiode and its electric field profile in the device are shown in Figure 4.1.

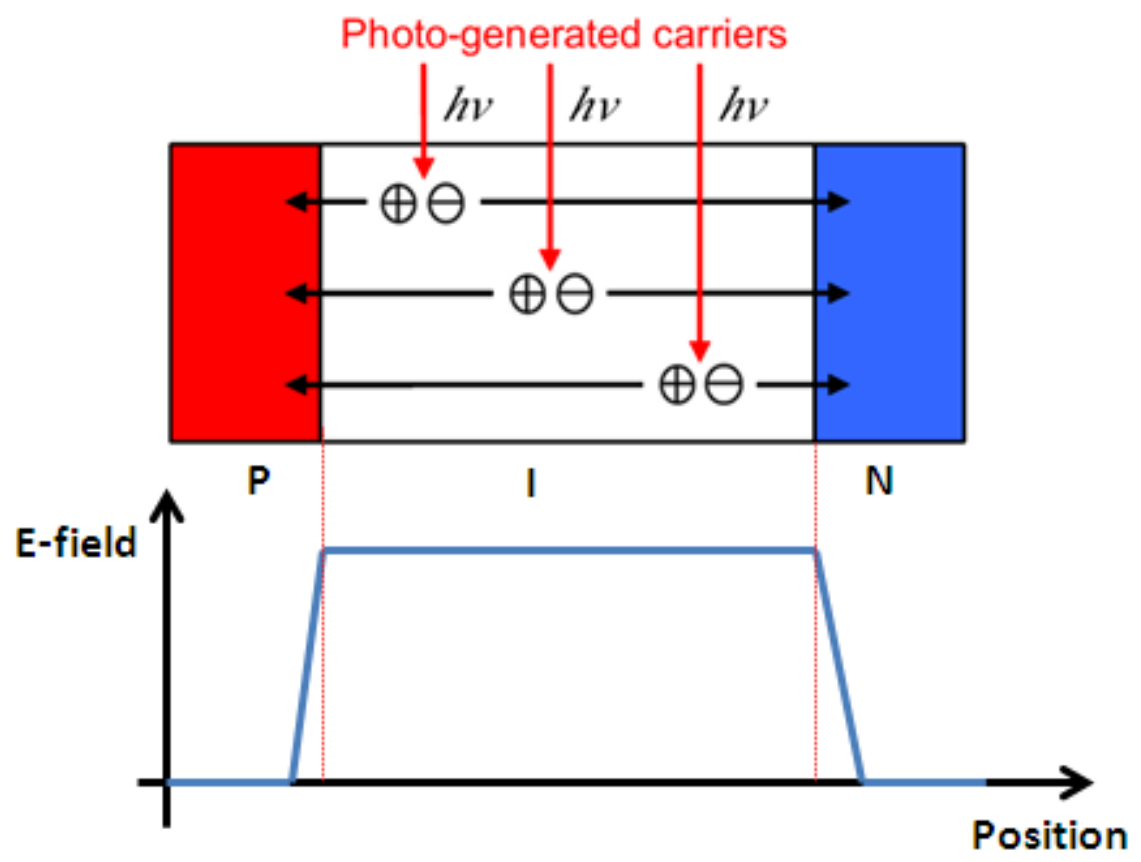

Figure 4.1 Device structure and electrical field distribution of PIN photodiodes

The PIN photodiode consists of an intrinsic layer, sandwiched between heavily doped n-type and p-type layers. In most cases, the intrinsic region is used as the absorption region and has a smaller band gap than the n- or p-type regions. When a reverse bias is 
applied, most of the electric field is within the intrinsic layer. Due to the low background doping level in i-region, the free carriers can usually be completely depleted at a zero or low reverse bias. The electron-hole pairs generated by light absorption in this region are swept across the region by the electric field, leading to current flow in the external circuit. Given a PIN photodiode with a fully depleted absorption region, the external quantum efficiency, $\eta_{\text {ext }}$, can be written as equation (4.1)

$$
\eta_{\text {ext }}=(1-R)\left(1-e^{-\alpha d}\right) \eta_{\text {coll }}
$$

where $\mathrm{d}$ is the width of the absorption region, $\alpha$ is the absorption coefficient, $\mathrm{R}$ is the reflectivity at the surface, and $\eta_{\text {coll }}$ is the carrier collection efficiency. Carrier collection efficiency is usually smaller than one, due to the fact that photo generated carriers may recombine before collected at the contact.

\subsection{Dark Current}

Strictly speaking, the dark current is measured when there is no light incident on the device (zero background radiation). However, it cannot be realized since zero background radiation would never exist. The definition I use here is this: whenever the background level is low enough to have negligible effect on the photodiode's current, I would define that current as the dark current of the photodiode. To achieve this condition, a cold shield is used to minimize any stray infrared radiation incident on the device.

The dark current-voltage characteristics of the devices are measured using HP 4145B semiconductor parameter analyzer. When measuring the dark current at various temperatures, the photodiodes would be placed in a cryostat and the cryostat windows would be covered with aluminum foil to prevent any light from entering the cryostat. 
There are five primary mechanisms that contribute to the dark current in a reverse biased photodiode: diffusion current $\left(\mathrm{I}_{\mathrm{Diff}}\right)$, generation-recombination current $\left(\mathrm{I}_{\mathrm{GR}}\right)$, bandto-band tunneling current $\left(\mathrm{I}_{\mathrm{T}}\right.$, ), trap-assisted tunneling current $\left(\mathrm{I}_{\mathrm{TAT}}\right)$ and surface recombination current $\left(\mathrm{I}_{\text {surf }}\right)$ [104-106]. My measurements in the next chapter show that the surface recombination current is not important in these devices. As a result, I do not include it in the modeling for this dissertation.

\subsubsection{Diffusion Current}

Diffusion current is one of the most fundamental mechanisms that contribute to the dark current, being present in all kinds of photodiodes. It is due to the thermally generated minority carriers in the quasi-neutral regions (p-region or n-region) that diffuse across the junction. These minority carriers are usually within a diffusion length away from the depletion region. The diffusion current is given by [104-106]:

$$
\mathrm{I}_{\text {Diff }}=A n_{\mathrm{i}}^{2} \sqrt{\mathrm{ekT}}\left(\frac{1}{\mathrm{~N}_{\mathrm{A}}} \sqrt{\frac{\mu_{\mathrm{e}}}{\tau_{\mathrm{e}}}}+\frac{1}{\mathrm{~N}_{\mathrm{D}}} \sqrt{\frac{\mu_{\mathrm{h}}}{\tau_{\mathrm{h}}}}\right)\left(\mathrm{e}^{\mathrm{eV} / \mathrm{nkT}}-1\right)
$$

where $\mathrm{A}$ is the cross section area of the device, $\mathrm{n}_{\mathrm{i}}$ is the intrinsic carrier concentration of the semiconductor material used for $\mathrm{p}$ - or $\mathrm{n}$-region, e is the electron charge, $\mathrm{k}$ is the Boltzmann constant, $\mathrm{T}$ is the temperature, $\mathrm{N}_{\mathrm{A}}$ and $\mathrm{N}_{\mathrm{D}}$ are the p- and n-type doping concentrations, respectively, $\mu_{\mathrm{e}}, \tau_{\mathrm{e}}$ and $\mu_{\mathrm{h}}, \tau_{\mathrm{h}}$ are the mobility and lifetime of electrons and holes, respectively, $\mathrm{V}$ is the bias voltage. The dynamic ideality factor $\mathrm{n}$ is 1 if the photodiode is diffusion current limited [107].

\subsubsection{Generation-recombination Current}

Generation-recombination (GR) current in photodiodes is due to defects in the depletion region. These defects can originate from either the material growth or the 
device fabrication. They act as intermediate states for carrier generation when the photodiodes are reverse biased, and act as intermediate states for carrier recombination when the photodiodes are forward biased. The generation-recombination current is given by [104-106]:

$$
\begin{gathered}
I_{G R}=\frac{\operatorname{Aen}_{i}^{a b s} d(V)}{\tau_{G R}} \frac{2 k T}{e\left(V_{b i}-V\right)} \sinh (-e V / 2 k T) f(b) \\
f(b)=\int_{0}^{\infty} \frac{d u}{u^{2}+2 b u+1} \\
b=e^{-q V} / 2 k T \cosh \left(\frac{E_{t}-E_{i}}{k T}\right)
\end{gathered}
$$

where $\mathrm{V}_{\mathrm{bi}}$ is the built-in potential, $\mathrm{d}$ is the depletion width, $\mathrm{n}_{\mathrm{i}}^{\text {abs }}$ is the intrinsic carrier concentration of absorption region, $\tau_{\mathrm{GR}}$ is the generation-combination lifetime. The dynamic ideality factor $\mathrm{n}$ is 2 if the photodiode is generation-recombination current limited [107].

\subsubsection{Band to Band Tunneling Current}

The band to band tunneling current originates from carriers that tunnel directly from the valence band to the conduction band. Generally, this type of current is apparent at large reverse bias because of the higher probability tunneling under the high electric field in the depletion region, and has negligible influence on the forward bias characteristics. The band tunneling current has a very small dependence on temperature which originates from the change in band gaps with temperature [53]. The band to band tunneling current can be calculated with following equation if the potential barrier is modeled as triangular [104-106]. 


$$
I_{T}=\frac{A e^{3} F(V) V}{4 \pi^{2} \hbar^{2}} \sqrt{\frac{2 m_{T}}{E_{g}}} \exp \left(-\frac{4 \sqrt{2 m_{T} E_{g}^{3}}}{3 e \hbar F(V)}\right)
$$

where $\mathrm{F}$ is the electric field, $\mathrm{h}$ is the Planck constant, $\mathrm{m}_{\mathrm{T}}$ is the effective tunneling mass, $\mathrm{E}_{\mathrm{g}}$ is the band gap or effective band gap of the depletion region.

\subsubsection{Trap-assisted Tunneling Current}

Trap-assisted tunneling (TAT) is a two-step tunneling process where carriers tunnel across the junction via trap states in or near depletion region. This tunneling consists of a thermally activated transition from the valence band to the trap site, then tunneling into an empty state in the conduction band. Similar to the band to band tunneling, this tunneling current has a very small temperature dependence and insignificant influence on the forward bias characteristics. The trap assisted tunneling current can be calculated by [104-106]:

$$
I_{T A T}=\frac{A e^{2} m_{T} V M^{2} N_{t}}{8 \pi \hbar^{3}\left(E_{g}-E_{t}\right)} \exp \left(-\frac{4 \sqrt{2 m_{T}\left(E_{g}-E_{t}\right)}}{3 e \hbar F(V)}\right)
$$

where $N_{t}$ is the activated trap density, $E_{t}$ is trap energy location, measured from the valence band edge, and $\mathrm{M}^{2}$ is a matrix element associated with trap potential.

All the formulas described above are for bulk PIN photodiodes, which would be different for the real structure of a photodiode with InGaAs/GaAsSb type-II MQWs as absorption region. However, for numerically modeling the real device's current, I can still get good fitting using these formulas to describe the photodiodes in this work as shown in next chapter. 


\subsection{Spectral Responsivity}

The spectral response of the devices discussed in this dissertation is measured by using a Nicolet Magna-II Fourier transform infrared (FTIR) spectrometer and a blackbody source at $700{ }^{\circ} \mathrm{C}$.

Figure 4.2 shows a schematic diagram of the FTIR spectrometer [108]. The mechanism of FTIR spectrometer is based on Michelson interferometer. Light from the infrared source is collimated and directed to a beam splitter. Ideally, 50\% of the light is transmitted towards the fixed mirror and 50\% of the light is reflected toward the moving mirror. During the motion of the moving mirror, the difference in optical path length between the two arms to the interferometer is recorded. An interferogram is obtained by moving the mirror and recording the signal from the detector for various positions of the mirror (with various optical path length differences). The Fourier transform of the interferogram is used to provide the actual spectrum of the target radiation. 


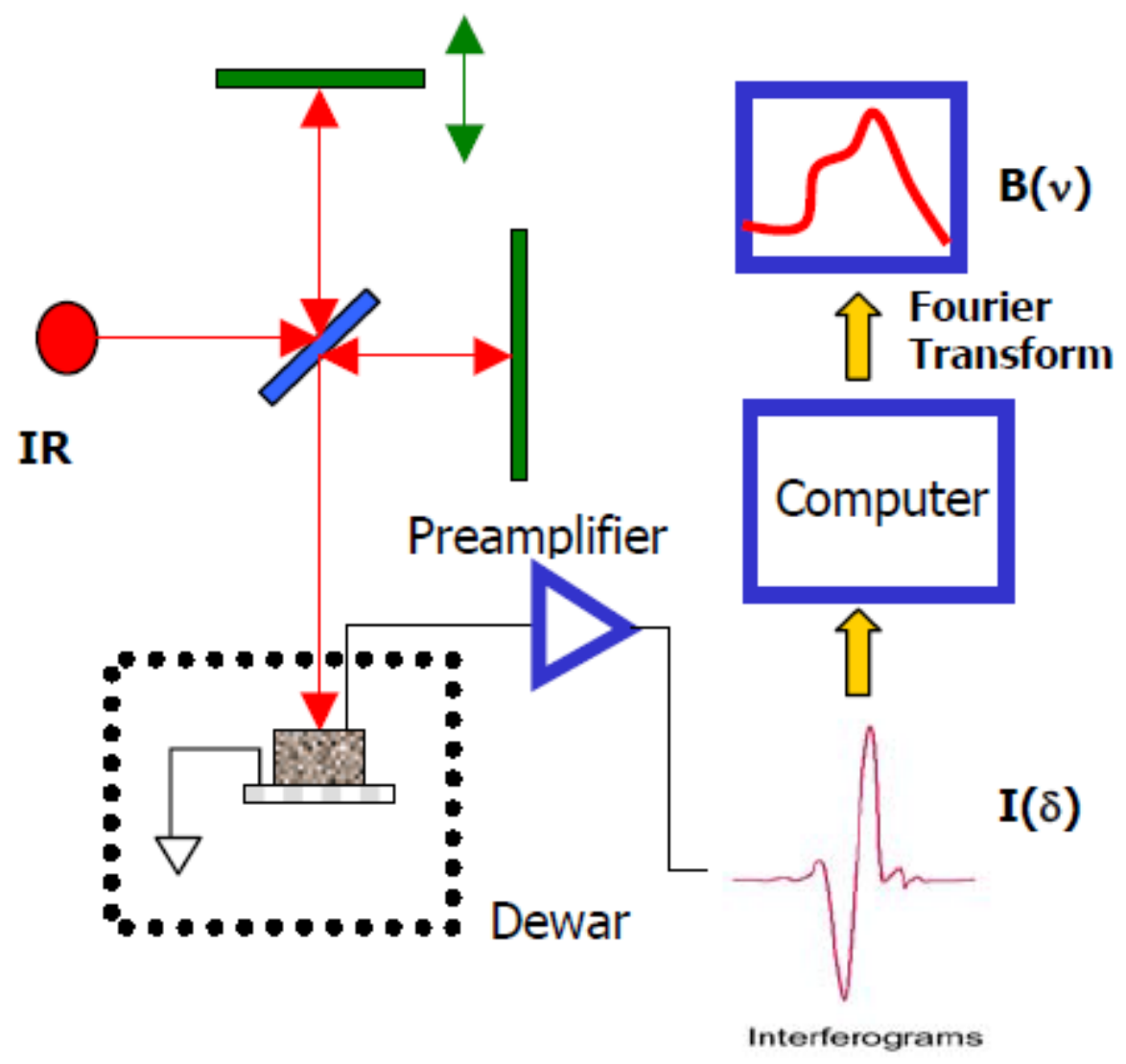

Figure 4.2 Principle of FTIR operation

The FTIR system enables users to connect an external detector unit, instead of using the built-in standard broad band DTGS (KBr window) detector. By collecting the same infrared source spectrum using two different detectors, we can extract the relative responsivity of the detector under test, along with its cut-off wavelength information. Here is the procedure to measure the relative responsivity of the SWIR and MWIR photodetectors in this dissertation. First, we use the FTIR built-in standard DTGS detector to the get the spectrum of the infrared light source as below in Figure 4.3. 


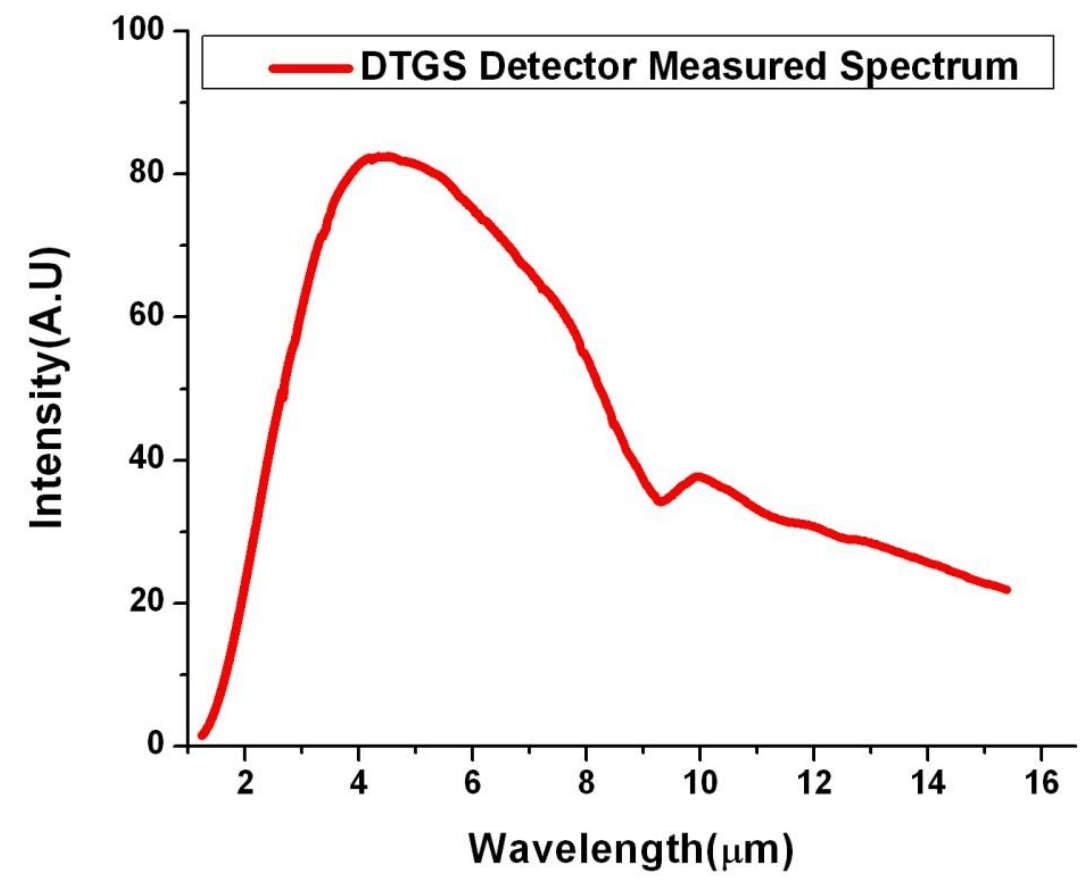

Figure 4.3 The spectrum of the infrared light measured by the DTGS detector

Then an external detector under test is used to replace the DTGS detector and measure the same spectrum of the infrared light source in FTIR again, which is shown in Figure 4.4 . 


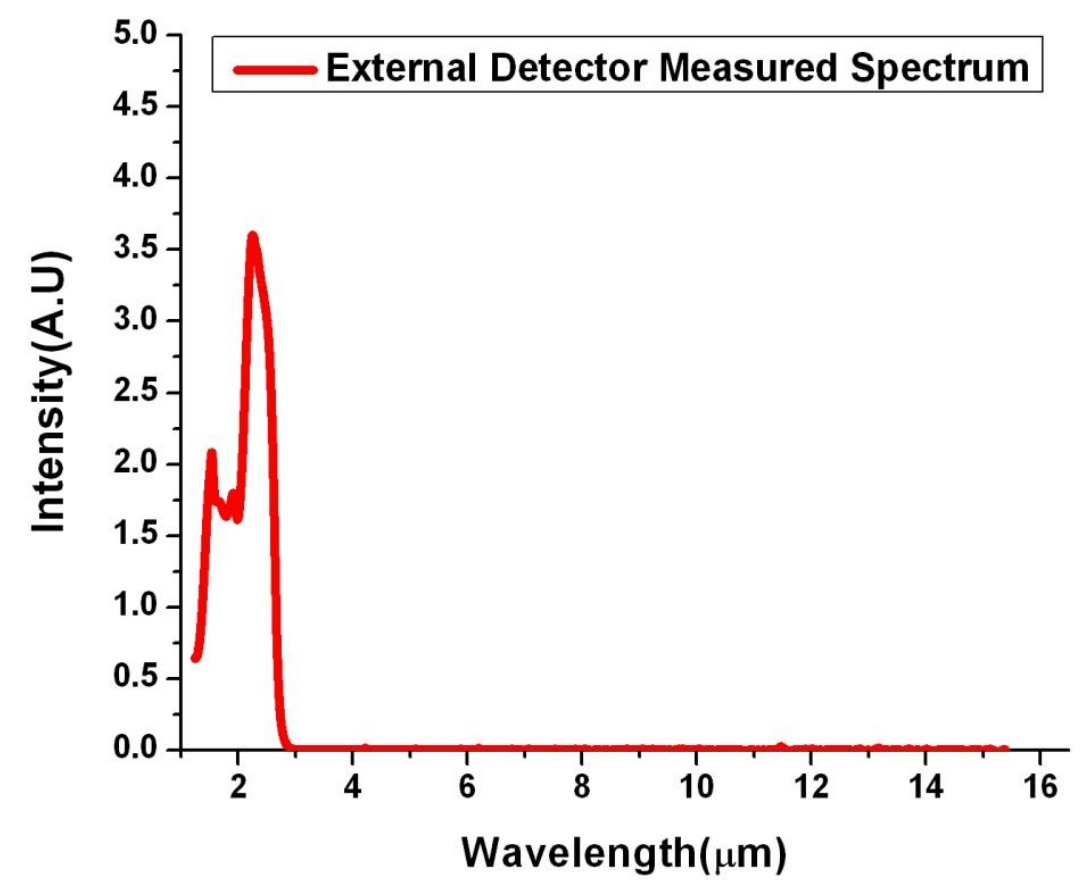

Figure 4.4 The spectrum of the infrared light measured by the external detector

Here a Stanford Research Systems (SRS) 570 low-noise current preamplifier is used to amplifier the signal out of the external detector during the measurement of the relative spectral response. With these two measured spectrum, the relative responsivity of the external detector to be tested can be extracted if we divide the first spectrum by the second spectrum as shown in Figure 4.5. 


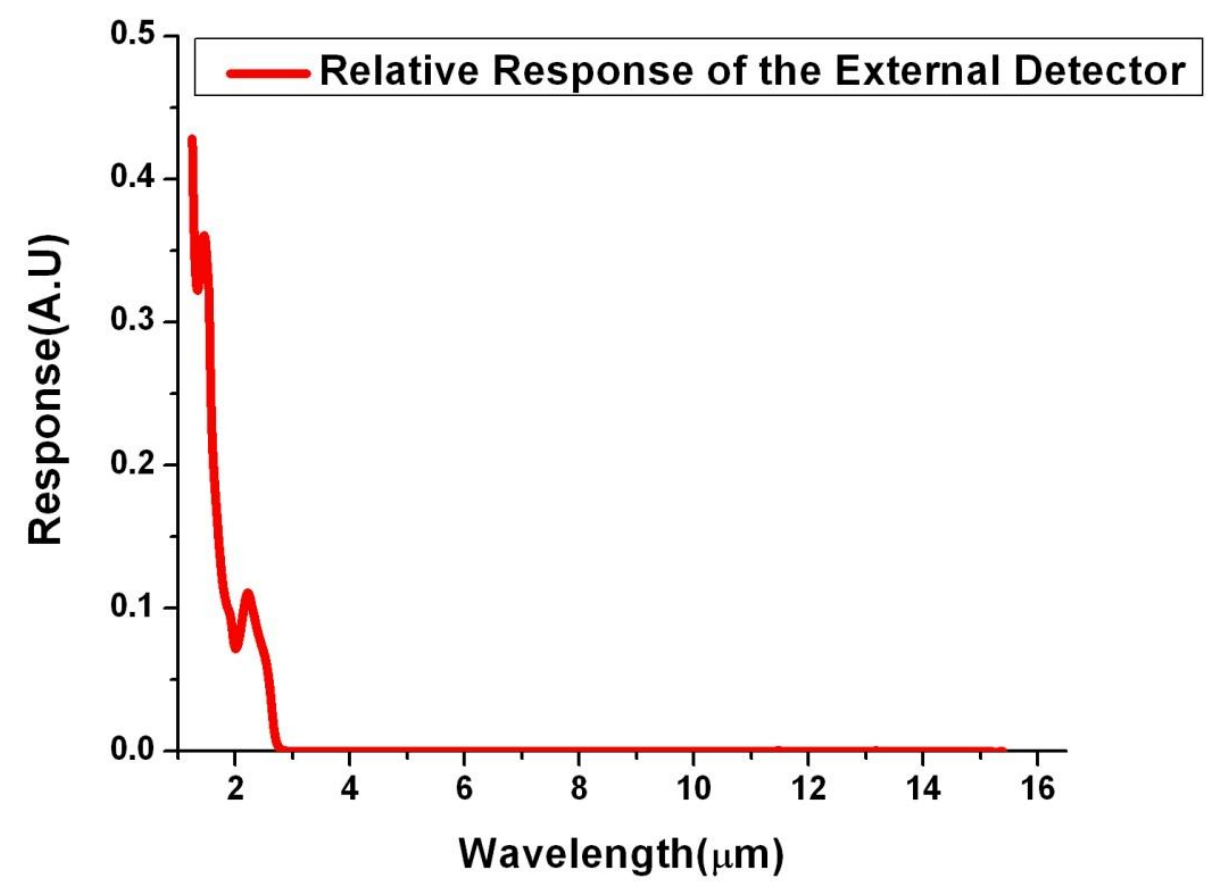

Figure 4.5 The relative responsivity of the external detector to be tested

I assume the DTGS detector has flat response over the wavelength range of interest, since it is a thermal detector. This assumption may not be true for wavelength below $1.8 \mu \mathrm{m}$, and it may generate erroneous spectrum for that wavelength range. Therefore, for the near infrared range (NIR) range, I do not use this method to measure the responsivity of the photodetectors. The responsivity below $1800 \mathrm{~nm}$ can be measured by comparing the photo response of the device under test with that of a calibrated commercial photodiode (such as InGaAs photodiodes). A tungsten-halogen lamp filtered by a grating monochromator is used to generate a monochromatic light (from $1000 \mathrm{~nm}$ to $1800 \mathrm{~nm}$ ). To remove the higher order short wavelengths generated in the monochromator, a long pass optical filter with a cut-off wavelength (1000 $\mathrm{nm}$ for example) is used to remove the visible light. 
The absolute responsivity of the devices is calibrated by using a blackbody source maintained at $700{ }^{\circ} \mathrm{C}$ with a chopper at a modulation frequency of $140 \mathrm{~Hz}$, a current preamplifier, and a Fast Fourier Transform (FFT) spectrum analyzer. In this dissertation, an 1800-nm long pass filter is placed between the blackbody source and the device to eliminate the contribution to the responsivity from spatially direct absorption of the InGaAs/GaAsSb type-II multiple quantum wells photodiodes. The detail of calibration process of the absolute responsivity will be discussed below.

Based on the Planck's radiation law, the spectral distribution of energy in the blackbody radiation is described as in formula 4.6 [35].

$$
\mathrm{N}(\lambda, \mathrm{T})=\frac{2 \pi h c^{2}}{\lambda^{5}} \frac{1}{\mathrm{e}^{\mathrm{hc}}}
$$

Here $\lambda$ is the wavelength, $\mathrm{T}$ is the temperature, $\mathrm{k}$ is the Boltzmann's constant, $\mathrm{c}$ is the speed of the light in vacuum, and $\mathrm{N}(\lambda, T)$ is the energy per unit time (or the power) radiated per unit area of emitting surface in the normal direction per unit solid angle per unit wavelength by a black body at temperature $\mathrm{T}$. The spectrum of blackbody radiation $700{ }^{\circ} \mathrm{C}$ is shown in Figure 4.6. The reason we use $700{ }^{\circ} \mathrm{C}$ as the radiation temperature of blackbody is that the maximum radiation produced by the blackbody is at wavelength of around $3 \mu \mathrm{m}$ for that temperature based on Wien's displacement law [35]. 


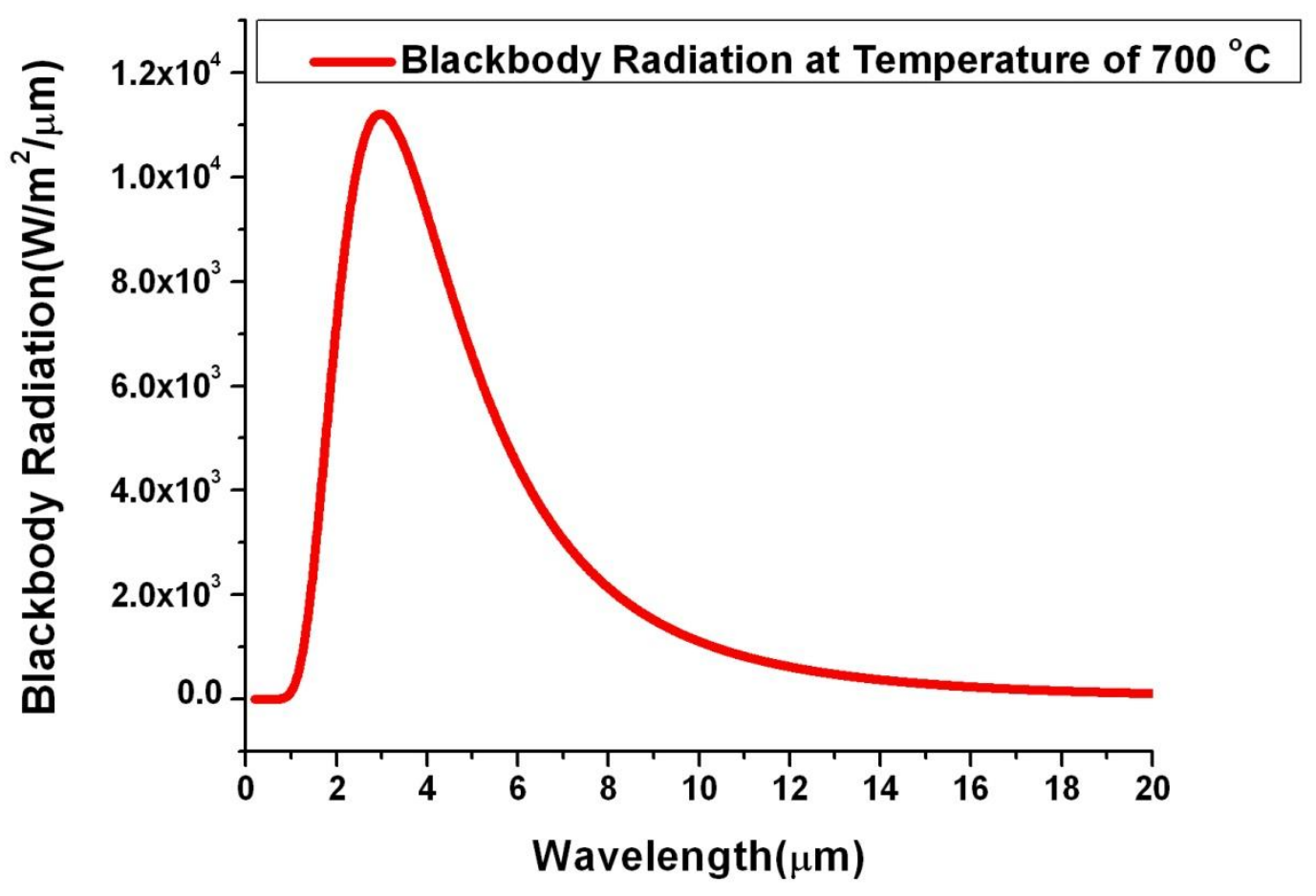

Figure 4.6 The spectral distribution of energy in the black body radiation at temperature of $700{ }^{\circ} \mathrm{C}$

The signal from the blackbody was modulated at a frequency of $140 \mathrm{~Hz}$ to get rid of the background radiation coming from the environment. The power density from blackbody can be calculated using the following equation (4.7).

$$
\mathrm{M}\left(\lambda_{1}, \lambda_{2}, \mathrm{~T}\right)=\int_{\lambda_{1}}^{\lambda_{2}} \mathrm{~N}(\lambda, \mathrm{T}) \mathrm{d} \lambda
$$

The power incident $\mathrm{P}_{\mathrm{in}}$ on the device is given by equation (4.8).

$$
\mathrm{P}_{\text {inc }}=\left[M\left(\lambda_{1}, \lambda_{2}, T\right)\right] \times \frac{\frac{\pi}{4} D_{\text {ap }}^{2}}{\pi d^{2}} \cdot T_{\text {ZnSe }} \cdot T_{i} \cdot A_{\text {det }} \cdot M F
$$

where $\mathrm{T}$ is the blackbody radiation temperature that is $700{ }^{\circ} \mathrm{C}, \mathrm{D}_{\mathrm{ap}}$ is the aperture on the blackbody source, $\mathrm{d}$ is the distance between the photodiodes and the blackbody source, $\mathrm{T}_{\mathrm{ZnSe}}$ is the transmission of the window, $\mathrm{T}_{\mathrm{i}}$ is the transmission of the $1800 \mathrm{~nm}$ long pass 
filter, $\mathrm{A}_{\text {det }}$ is the active area on the device mesa top and MF is the modulation factor for square-wave input signal that is 0.45 for the chopper we use.

The total current under radiation from the photodiode is measured by using a current preamplifier and a Fast Fourier Transform (FFT) spectrum analyzer. With the total current measured, the ratio $\beta$ between the relative responsivity $R_{a b}$ and absolute responsivity $R_{\text {re }}$ can be calculated in equation (4.9) as below. With the ratio $\beta$, the responsivity as a function of wavelength can be determined finally.

$$
\beta=\frac{\mathrm{R}_{\mathrm{ab}}}{\mathrm{R}_{\mathrm{re}}}=\frac{\text { total current under radiation }}{\int_{\lambda_{1}}^{\lambda_{2}}[\mathrm{~N}(\lambda, \mathrm{T})] \frac{\frac{\pi}{4} \mathrm{D}_{\mathrm{ap}}^{2}}{\pi \mathrm{d}^{2}} \cdot \mathrm{T}_{\mathrm{ZnSe}} \cdot \mathrm{T}_{\mathrm{i}} \cdot \mathrm{A}_{\mathrm{det}} \cdot \mathrm{MF} \cdot \mathrm{R}_{\mathrm{re}}(\lambda) \mathrm{d} \lambda}
$$

\subsection{Dark Noise}

The random nature of the motion of charged carriers leads to noise in the detector signal. The total noise is the root sum of squares (or RSS) of all the contributing noise sources, as the different noise source relevant to my detectors are discussed below [109].

1) Thermal Noise: Thermal noise exists in all resistors at temperature above zero Kelvin, and it stems from the random thermal motion of carriers in a material. The expression is given by

$$
i_{\text {thermal }}^{2}=4 \frac{h f}{e^{h f / k T}-1} \frac{\Delta f}{R} \sim \frac{4 k T \Delta f}{R}
$$

where $\mathrm{k}$ is the Boltzmann's constant, $\mathrm{h}$ is the Planck constant, $\mathrm{T}$ is the temperature, $\mathrm{R}$ is the resistance, $\mathrm{f}$ is the noise measurement frequency and $\Delta f$ is the noise measurement bandwidth. Since in reality, $\mathrm{hf} \ll \mathrm{kT}$ is always satisfied, the quantum factor $\frac{\mathbf{h f}}{\mathbf{e}^{\mathbf{h f} / \mathbf{k T}}-\mathbf{1}}$ reduces to $\mathrm{kT}$ in equation (4.10). 
2) Shot Noise: This noise originates from the discrete nature of the electric charge. When viewed at the electron level, the current in the detector does not flow in a smooth, uniform way. Even for constant average (DC) current, the root mean square deviation (or RMSD) in the current about this average value due to fluctuations at electron level still exists. The expression for shot noise is given as below:

$$
i_{\text {shot }}^{2}=2 e I \Delta f
$$

where $e$ is the electron charge, and $I$ is the average current created by the electron stream.

3) Generation-recombination Noise: Generation-recombination (g-r) noise in semiconductor originates from traps that randomly capture and emit carriers; therefore, there are fluctuations in the number of carriers available for current transport. The forms of transitions which occur in a semiconductor are electrons trapped by empty traps, electrons released by full traps, holes trapped by full traps, holes released by empty traps. The power spectrum density of g-r noise is proportional to the number of traps.

4) 1/f Noise (Flicker Noise): This noise has power spectral density inversely proportional to the frequency and is only present for non-zero bias operation. It usually shows up as a low-frequency phenomenon, as the higher frequencies are overshadowed by other noise sources. Although the cause of $1 / \mathrm{f}$ noise is not fully understood, it can show up due to various reasons, such as mobility fluctuations, surface related issues and so on. Since the response and noise of photodiodes in this dissertation are measured under low frequency such as $140 \mathrm{~Hz}$, this noise source may also contribute. 
The dark noise of the photodiodes is characterized by using the similar procedure as the responsivity. To obtain this data, the device is mounted on a cold finger inside a cryostat and the cryostat chamber was covered to eliminate stray light from shining on the device. The noise current from the device is amplified by using a pre-amplifier. An FFT spectrum analyzer is used to view the noise spectrum density.

\subsection{Specific Detectivity}

The signal to noise ratio is often used as a figure of merit between different detector structures. This comparison is complicated by the fact that the signal to noise ratio is a function of detector area and the bandwidth. To combat this, the specific detectivity is typically used as a means of comparison. The specific detectivity is defined as the reciprocal of noise equivalent power, normalized per unit area and bandwidth and can be calculated from the noise current spectrum density and the responsivity, with the equation (4.12) below.

$$
D^{*}=\frac{R \sqrt{A}}{\text { noise } / \sqrt{\Delta f}}
$$

where $\mathrm{A}$ is the device area, $\mathrm{R}$ is the responsivity, $\Delta \mathrm{f}$ is the bandwidth. And common units of the specific detectivity are $\mathrm{cm} \cdot \mathrm{Hz}^{0.5} / \mathrm{W}$, also called Jones.

\subsection{Summary}

In this chapter, the parameters used to characterize the infrared photodiodes are reviewed, as these parameters are important in the following chapters. The measurements of each parameter are also presented, and the major components for the dark current and dark noise for an infrared photodiode are also discussed. With these parameters, the performance of the InP based type-II quantum wells photodiodes will be characterized in next chapter. 


\section{Chapter 5: InGaAs-GaAsSb Type-II Quantum Wells PIN Photodiodes}

\subsection{Introduction}

In chapter 3, I discussed the design of InGaAs/GaAsSb type-II quantum wells as the absorption region of infrared photodiodes. Based on the simulation results, a thicker InGaAs layer compared with the GaAsSb layer is desirable in terms of achieving the longer absorption wavelength and larger wave function overlap simultaneously. Furthermore, when strain compensation is used, the GaAsSb layer should be compressively strained while the InGaAs layer is tensile strained in order to detect longer wavelength infrared light.

In this chapter, I will study how the performance of these type-II photodiodes changes as the detection wavelength increases by comparing three different structures. The first sample (Sample A) consists of 100 pairs of lattice matched $7 \mathrm{~nm} \mathrm{In}_{0.53} \mathrm{Ga}_{0.47} \mathrm{As} / 5 \mathrm{~nm}$ $\mathrm{GaAs}_{0.5} \mathrm{Sb}_{0.5}$ quantum wells as absorption region. This is similar to previously reported structures [34]; the thicker InGaAs layer will lead to a longer detection wavelength. The second sample (Sample B) consists of 100 pairs of strain compensated $7 \mathrm{~nm}$ $\mathrm{In}_{0.34} \mathrm{Ga}_{0.66} \mathrm{As} / 5 \mathrm{~nm} \mathrm{GaAs} \mathrm{s}_{0.25} \mathrm{Sb}_{0.75}$ quantum wells as absorption region, in which case the GaAsSb layers are compressively strained and the InGaAs layers are tensile strained. These two structures were designed to SWIR and MWIR detection respectively based on the design criteria concluded in Chapter 3. A third sample (Sample C) uses a new strain compensation scheme which would have potential advantages over Sample B. All those 
materials are epitaxially grown in an MBE reactor by IQE Inc, fabricated as discussed in the Appendix, and characterized as described in chapter 4.

\subsection{Lattice Matched Quantum Wells Photodiode (Sample A)}

The structure of Sample A is shown in Figure 5-1. An unintentionally doped 100 pairs of $7 \mathrm{~nm} \mathrm{In}_{0.53} \mathrm{Ga}_{0.47} \mathrm{As} / 5 \mathrm{~nm} \mathrm{GaAs}{ }_{0.5} \mathrm{Sb}_{0.5}$ quantum wells absorption region is placed between p-type and n-type layers. The transitions between the ground energy states in the latticed matched InGaAs and GaAsSb layer are expected to occur around $0.47 \mathrm{eV}$, corresponding to the transition wavelength of around $2.63 \mu \mathrm{m}$.

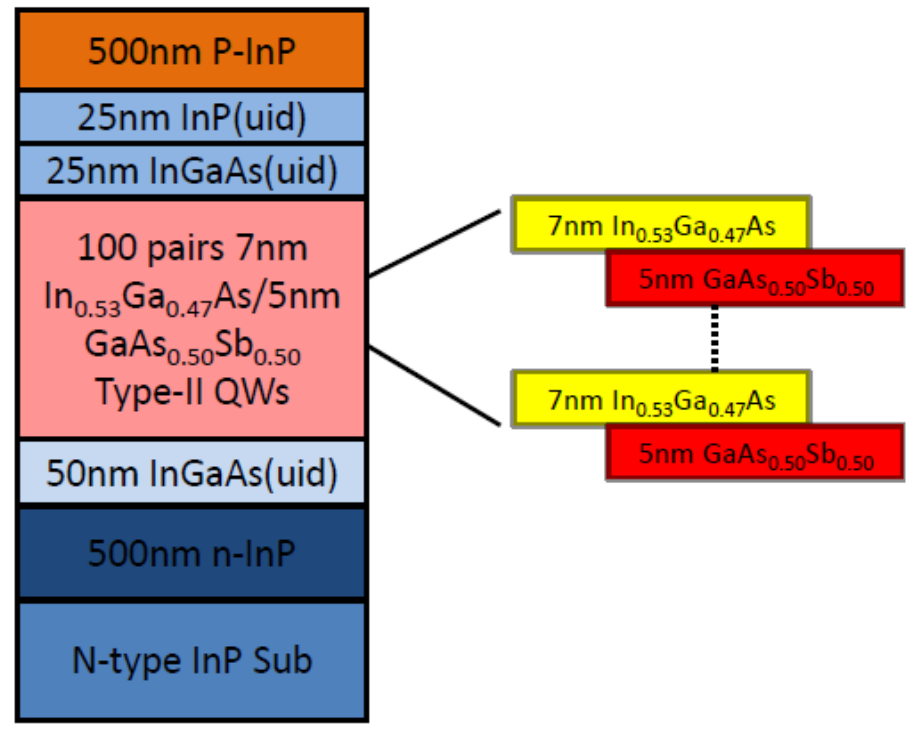

Figure 5.1 A schematic of the PIN device structure with type-II $7 \mathrm{~nm} \mathrm{In}_{0.53} \mathrm{Ga}_{0.47} \mathrm{As} / 5 \mathrm{~nm} \mathrm{GaAs} \mathrm{Ga}_{0.5} \mathrm{Sb}_{0.5}$ MQWs as absorption layer (Sample A)

Material composition and thickness of layers were verified using high resolution Xray diffraction (HRXRD) as shown in Figure 5-2. The appearances of a large number of satellite peaks suggest that structure is of high quality and abrupt interfaces exist between the quantum well layers. 


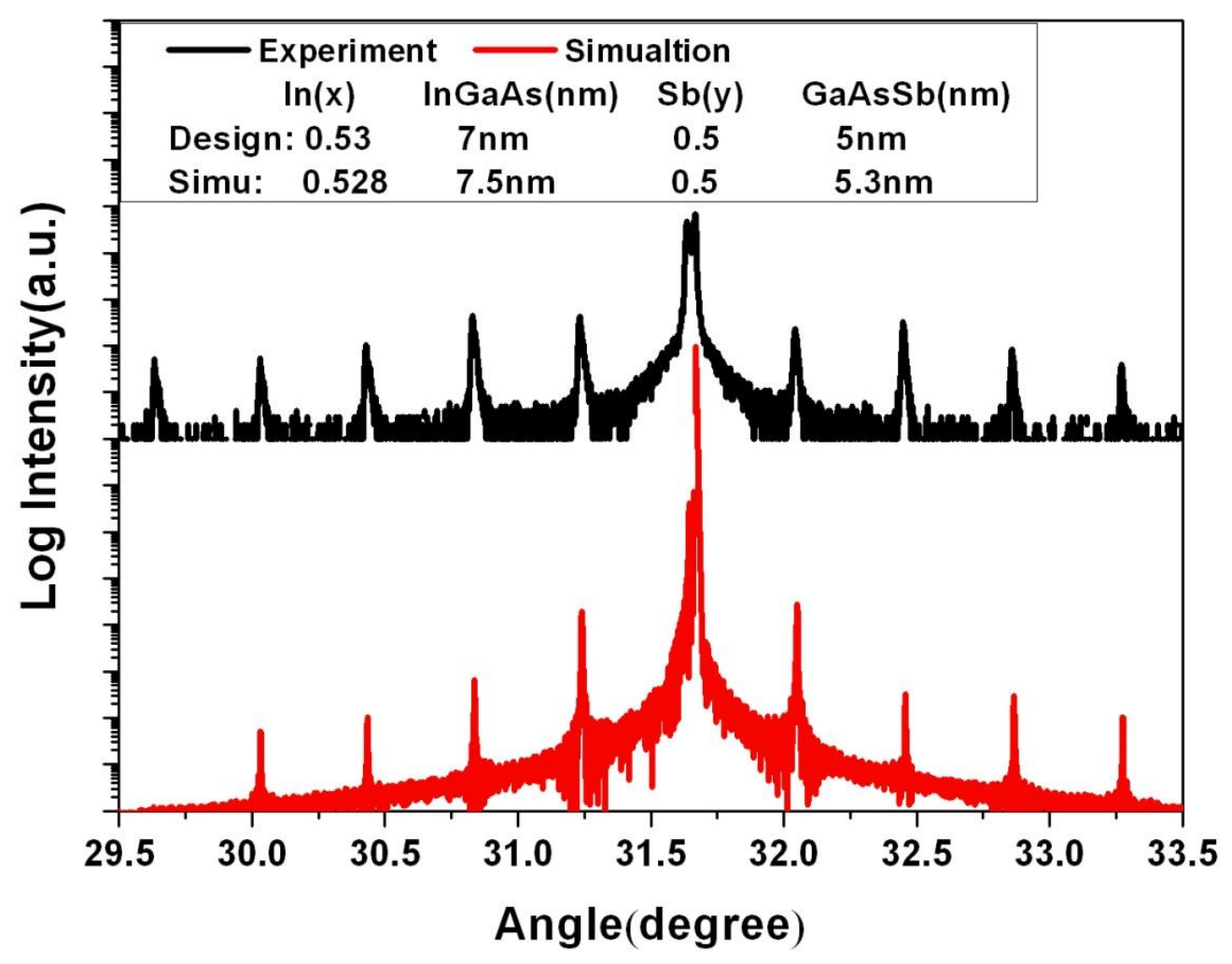

Figure 5.2 HRXRD data of Sample A with 100 pairs of $7 \mathrm{~nm} \mathrm{In}_{0.53} \mathrm{Ga}_{0.47} \mathrm{As} / 5 \mathrm{~nm} \mathrm{GaAs} \mathrm{ng}_{0.5} \mathrm{Sb}_{0.5} \mathrm{MQWs}$

\subsubsection{Characteristics}

The dark current-voltage (I-V) characteristics of Sample A are measured using an HP4145B semiconductor parameter analyzer. Twenty-nine out of thirty devices show very similar dark current with low variations. The dark current of a representative $180 \mu \mathrm{m}$ diameter device at different temperatures is shown in Figure 5-3. It has a dark current density of $1.66 \mathrm{~mA} / \mathrm{cm}^{2}$ at $290 \mathrm{~K}$ at $-0.5 \mathrm{~V}$, and decreases to $4.89 \mu \mathrm{A} / \mathrm{cm}^{2}$ at $200 \mathrm{~K}$. 


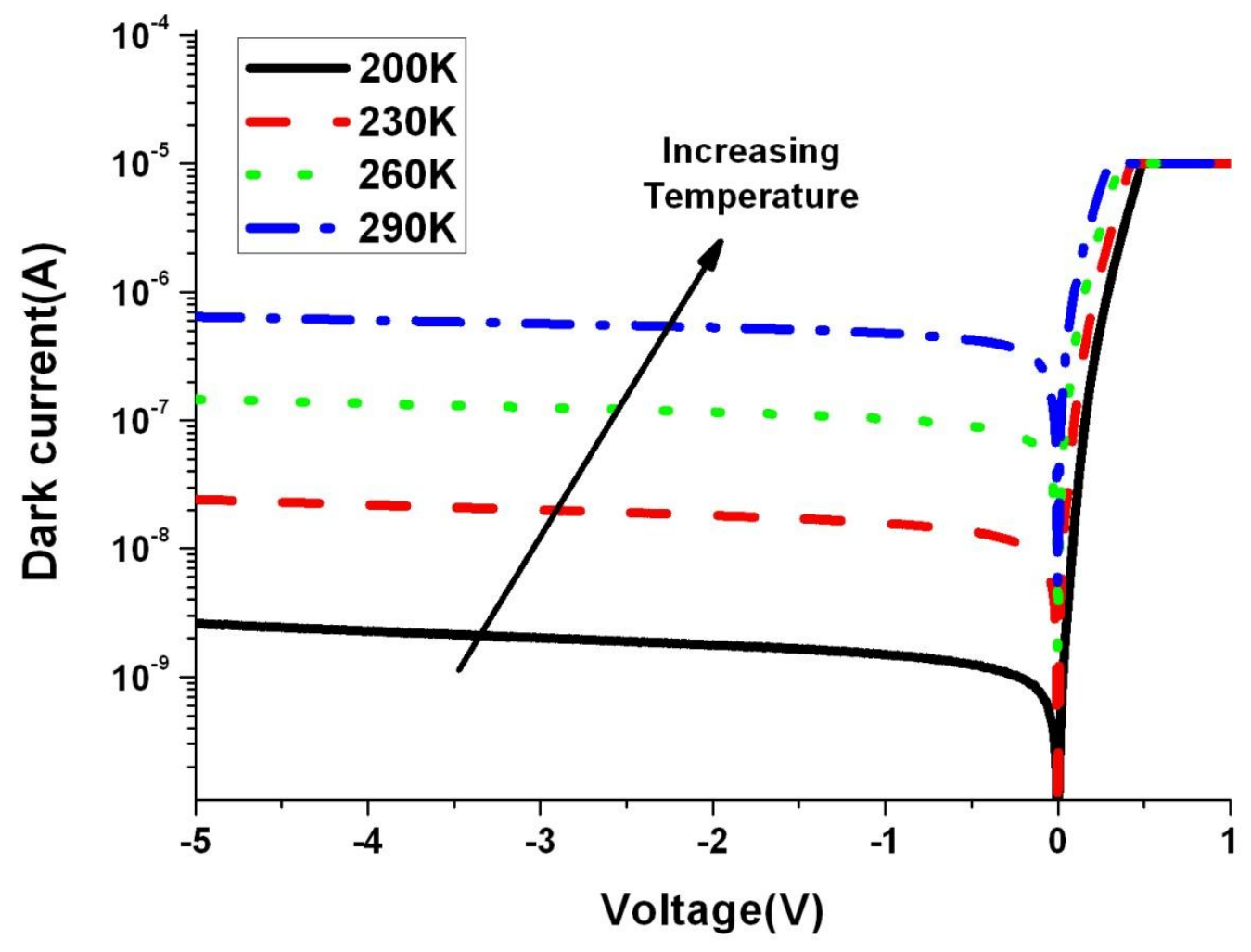

Figure 5.3 Dark current versus voltage for the $\mathbf{1 8 0} \mu \mathrm{m}$ diameter Sample A measured at different temperatures

To understand which components of the dark current are important in the performance of these devices, the dark current-voltage (I-V) characteristics at $290 \mathrm{~K}$ for different device areas of Sample A are shown in Figure 5-4. The fact that the dark current scales with device area as opposed to perimeter suggests that the dark current is dominated by the bulk component and not the surface leakage. 


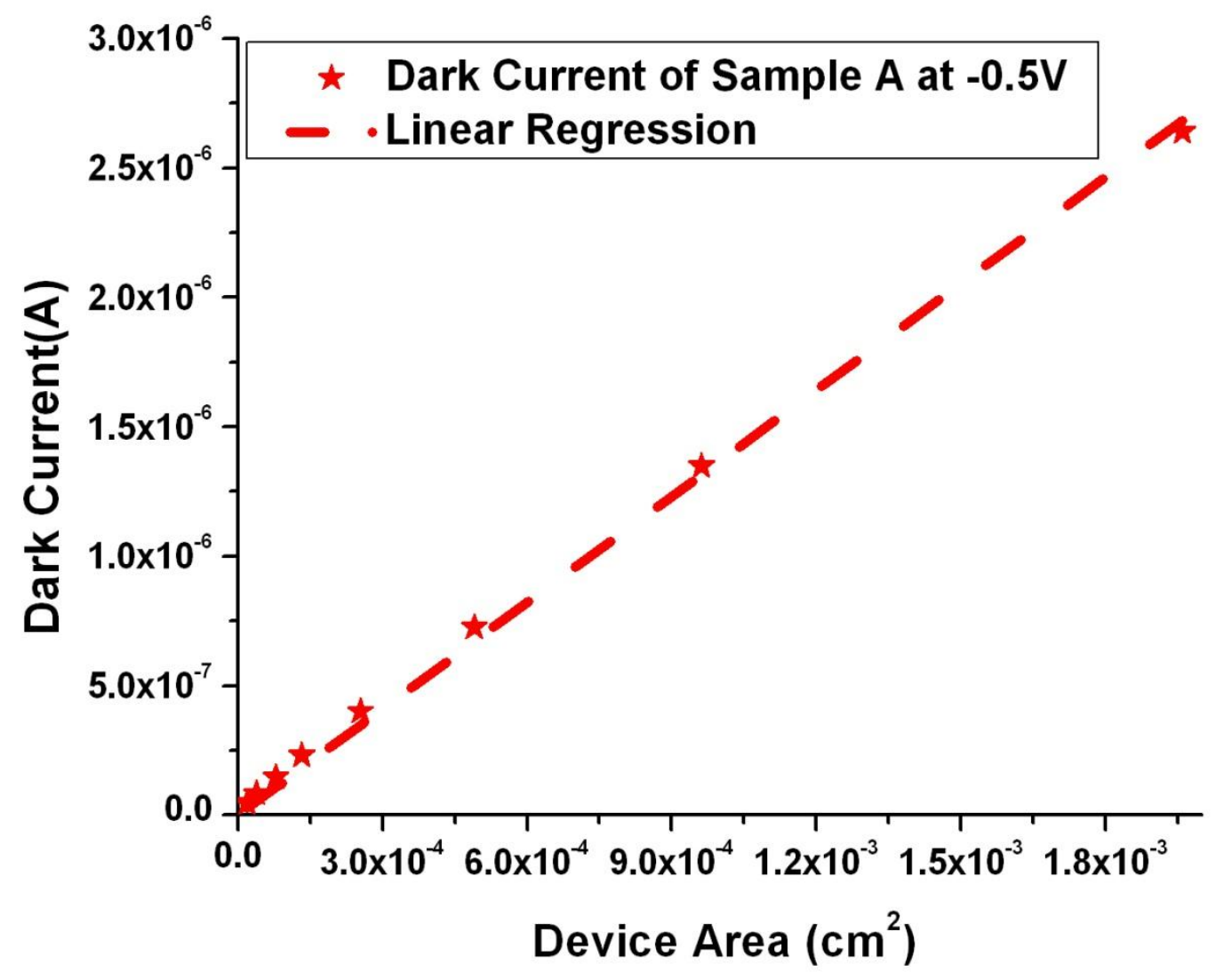

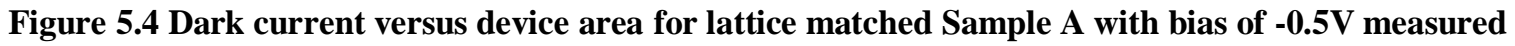
at $290 \mathrm{~K}$

An Arrhenius plot of the dark current for Sample A is shown in Figure 5-5. A good exponential fit to the data suggests that single activation energy (around $0.32 \mathrm{eV}$ ) dominates the dark current. The activation energy extracted in this way often represented the average energy of several traps rather than the signature of any single defect, especially for the multiple quantum wells structures, since the defect distribution is complicated [110]. 


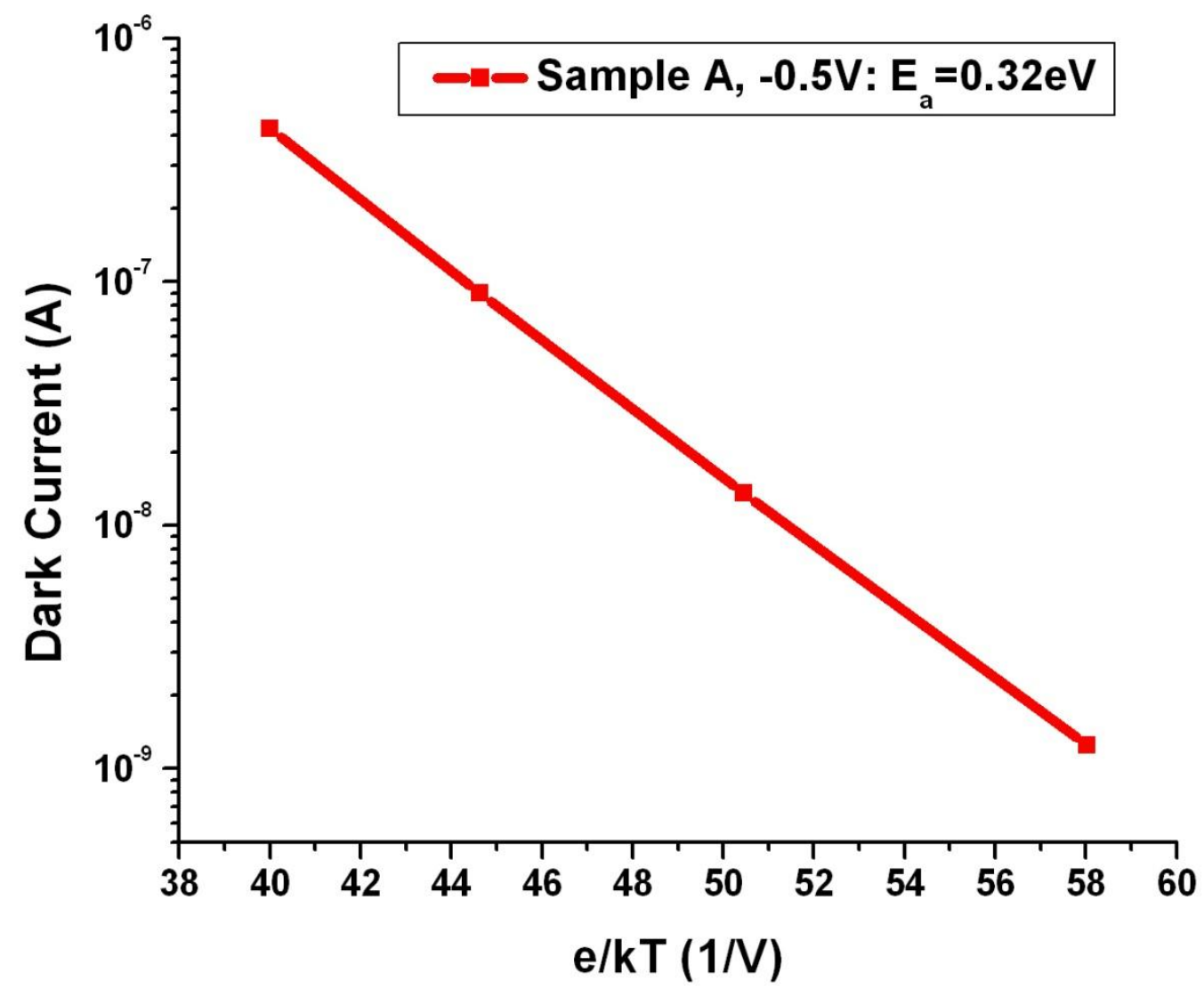

Figure 5.5 Arrhenius plot of the dark current for $180 \mu \mathrm{m}$ diameter Device A at reverse bias $\mathbf{- 0 . 5 V}$

Another figure of merit is the resistance-area product $\left(\mathrm{R}_{0} \mathrm{~A}\right)$, which is related to thermal noise as discussed in Chapter 4. $\mathrm{R}_{0} \mathrm{~A}$ is calculated from the low bias I-V data at different temperature for $180 \mu \mathrm{m}$ diameter device as shown in Figure 5.6. The $\mathrm{R}_{0} \mathrm{~A}$ at $290 \mathrm{~K}$ is $50 \mathrm{Ohm} \cdot \mathrm{cm}^{2}$, and it increases to $18,000 \mathrm{Ohm} \cdot \mathrm{cm}^{2}$ at $200 \mathrm{~K}$. These values are comparable with that of the commercially available relaxed $\operatorname{In}_{x} \mathrm{Ga}_{1-\mathrm{x}} \mathrm{As}(\mathrm{x}>0.53) \operatorname{InP}$ based PIN photodiodes with the similar cut-off wavelength [72] and much higher than other infrared technologies [36]. 


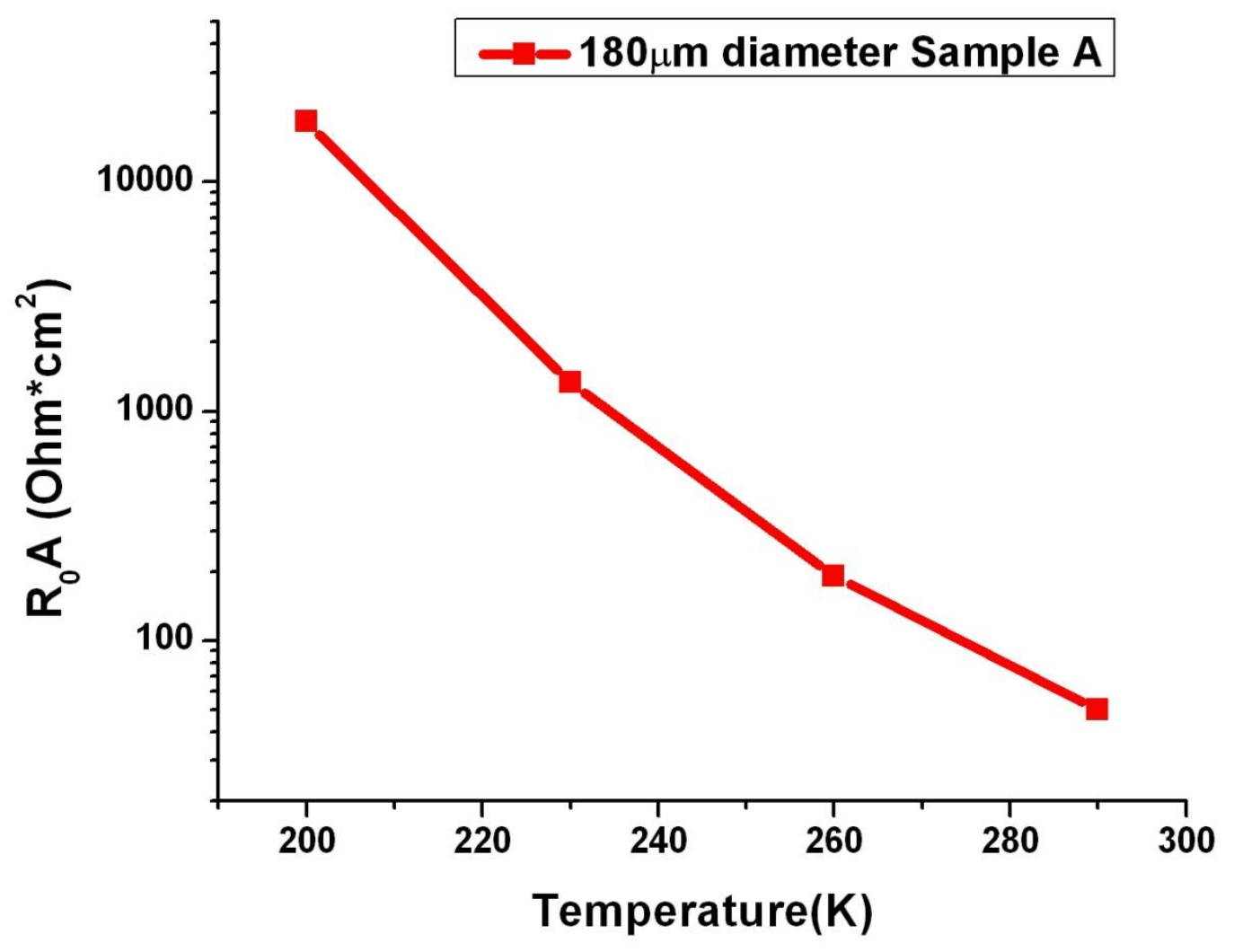

Figure 5.6 $\mathbf{R}_{0} A$ product for $180 \mu \mathrm{m}$ diameter Sample A at different temperatures

To understand the main contributions to this dark current, additional analysis is carried out. Given the fact that the dark current is dominated by bulk effects, the bulk based model described in Chapter 4 can be used to analyze the dark current performance of the photodiodes. Equations for the four main mechanisms: diffusion ( $\left.I_{\text {Diff }}\right)$, generationrecombination $\left(\mathrm{I}_{\mathrm{GR}}\right)$, band-to-band tunneling $\left(\mathrm{I}_{\mathrm{T}}\right.$, ), and trap-assisted tunneling $\quad\left(\mathrm{I}_{\mathrm{TAT}}\right)$ are provided again in Table 5-1. 
Table 5-1 Equations used to model the dark current of bulk PIN photodiodes

\begin{tabular}{|c|c|}
\hline Equation & Number \\
\hline $\mathrm{I}_{\mathrm{Diff}}=A n_{\mathrm{i}}^{2} \sqrt{\mathrm{ekT}}\left(\frac{1}{N_{\mathrm{A}}} \sqrt{\frac{\mu_{\mathrm{e}}}{\tau_{\mathrm{e}}}}+\frac{1}{\mathrm{~N}_{\mathrm{D}}} \sqrt{\frac{\mu_{\mathrm{h}}}{\tau_{\mathrm{h}}}}\right)\left(\mathrm{e}^{\mathrm{eV} / \mathrm{kT}}-1\right)$ & $(5.1)$ \\
\hline $\begin{array}{c}\mathrm{I}_{\mathrm{GR}}=\frac{\mathrm{Aen}_{\mathrm{i}}^{\mathrm{abs}} \mathrm{d}(\mathrm{V})}{\tau_{\mathrm{GR}}} \frac{2 \mathrm{kT}}{\mathrm{e}\left(\mathrm{V}_{\mathrm{bi}}-\mathrm{V}\right)} \sinh (-\mathrm{eV} / 2 \mathrm{kT}) \mathrm{f}(\mathrm{b}) \\
\text { where } \mathrm{f}(\mathrm{b})=\int_{0}^{\infty} \frac{\mathrm{du}}{\mathrm{u}^{2}+2 \mathrm{bu}+1}, \text { and } \mathrm{b}=\mathrm{e}^{-\mathrm{qV} / 2 \mathrm{kT} \cosh \left(\frac{\mathrm{E}_{\mathrm{t}}-\mathrm{E}_{\mathrm{i}}}{\mathrm{kT}}\right)}\end{array}$ & $(5.2)$ \\
\hline $\mathrm{I}_{\mathrm{T}}=\frac{\mathrm{Ae}^{3} \mathrm{~F}(\mathrm{~V}) \mathrm{V}}{4 \pi^{2} \hbar^{2}} \sqrt{\frac{2 \mathrm{~m}_{\mathrm{T}}}{\mathrm{E}_{\mathrm{g}}}} \exp \left(-\frac{4 \sqrt{2 \mathrm{~m}_{\mathrm{T}} \mathrm{E}_{\mathrm{g}}^{3}}}{3 \mathrm{e \hbar F}(\mathrm{V})}\right)$ & (5.3) \\
\hline $\mathrm{I}_{\mathrm{TAT}}=\frac{\mathrm{Ae}^{2} \mathrm{~m}_{\mathrm{T}} \mathrm{VM}^{2} \mathrm{~N}_{\mathrm{t}}}{8 \pi \hbar^{3}\left(\mathrm{E}_{\mathrm{g}}-\mathrm{E}_{\mathrm{t}}\right)} \exp \left(-\frac{4 \sqrt{2 \mathrm{~m}_{\mathrm{T}}\left(\mathrm{E}_{\mathrm{g}}-\mathrm{E}_{\mathrm{t}}\right)}}{3 \mathrm{e} \hbar \mathrm{F}(\mathrm{V})}\right)$ & (5.4) \\
\hline
\end{tabular}

In these equations, $\mathrm{n}_{\mathrm{i}}$ is the intrinsic carrier concentration of $\operatorname{InP}, \mathrm{n}_{\mathrm{i}}^{\text {abs }}$ is the intrinsic carrier concentration of absorption region, $\tau_{\mathrm{GR}}$ is the generation-recombination lifetime and is assumed to be $10 \mathrm{~ns}$ for the simulation, $\mathrm{m}_{\mathrm{T}}$ is the tunneling effective mass, $\mathrm{E}_{\mathrm{g}}$ is the effective band gap, which is transition energy between the lowest energy states in the InGaAs and GaAsSb, $N_{t}$ is the activated trap density, $E_{t}$ is trap energy location, measured from the valence band edge, $\mathbf{M}^{2}$ is a matrix element associated with trap potential, assumed to be $1 \times 10^{-23} \mathrm{eV}^{2} \mathrm{~cm}^{3}$ and all other parameters have been described in Chapter 4 .

Prior to fitting the experimental dark current curves, the effective band gap of the type-II absorption at different temperatures can be calculated using 6 band $\mathrm{k} \cdot \mathrm{p}$ method described in Chapter $3[84,85]$. The calculated effective band gap at each temperature is consistent to the cut-off wavelength based on optical response measurement shown in the 
next section. The individual current due to the each mechanism, the total fitted dark current and the total measured dark current are plotted in Figure 5-7. The parameters used for the fitting process at each temperature are listed in Table 5-2.

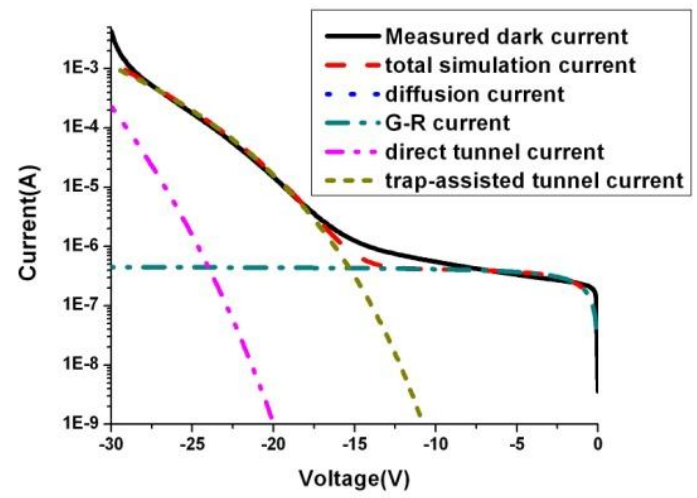

(a)

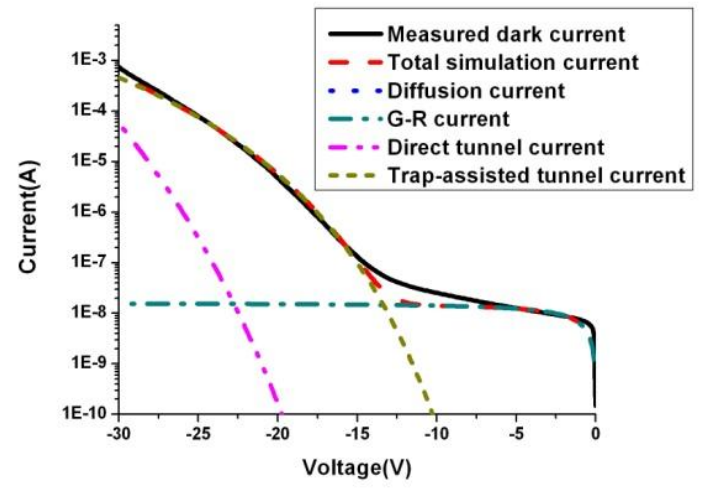

(c)

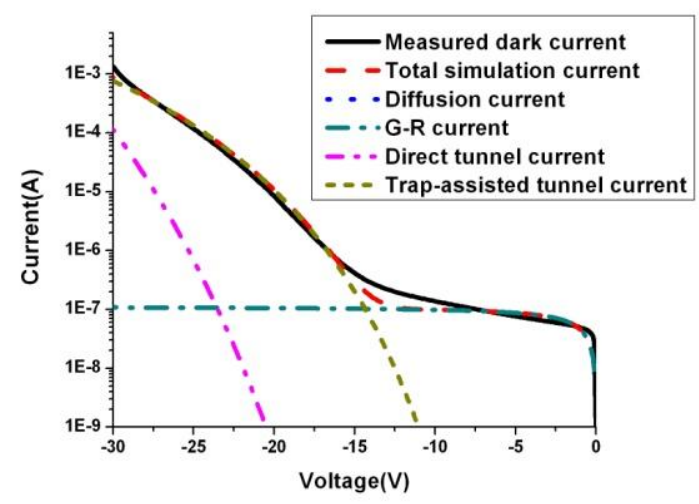

(b)

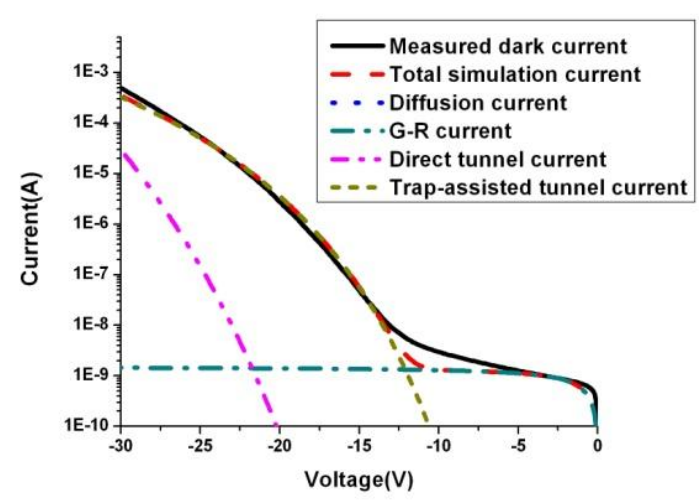

(d)

Figure 5.7 Experimental and simulated current considering diffusion, GR, band-to-band direct tunneling and trap-assisted tunneling for the lattice matched InGaAs-GaAsSb MQW photodiode with $130 \mu \mathrm{m}$ diameter investigated at (a) $290 \mathrm{~K}$, (b)260K, (c) $230 \mathrm{~K}$, (d) $200 \mathrm{~K}$. And the diffusion component is too low to be shown in the same plot.

Table 5-2 Parameters used to fitting the lattice matched InGaAs/GaAsSb p-i-n photodiodes.

\begin{tabular}{|l|l|l|l|l|}
\hline & $200 \mathrm{~K}$ & $230 \mathrm{~K}$ & $260 \mathrm{~K}$ & $290 \mathrm{~K}$ \\
\hline Effective band gap $(\mathrm{eV})$ & 0.51 & 0.50 & 0.49 & 0.47 \\
\hline Tunneling mass $\mathrm{m}_{\mathrm{T}}\left(\mathrm{m}_{\mathrm{e}}\right)$ & 0.08 & 0.08 & 0.08 & 0.08 \\
\hline Trap density $\mathrm{N}_{\mathrm{t}}\left(\mathrm{cm}^{-3}\right)$ & $1.636 \times 10^{12}$ & $1.655 \times 10^{12}$ & $1.93 \times 10^{12}$ & $2.34 \times 10^{12}$ \\
\hline Trap energy level $\mathrm{E}_{\mathrm{t}}\left(\mathrm{E}_{\mathrm{g}}\right)$ & 0.516 & 0.519 & 0.523 & 0.517 \\
\hline$n_{i}^{a b s}\left(\mathrm{~cm}^{-3}\right)$ & $1.440 \times 10^{10}$ & $1.46 \times 10^{11}$ & $1.005 \times 10^{12}$ & $2.931 \times 10^{12}$ \\
\hline
\end{tabular}


The strong agreement between the simulated and the experimental current confirm the validity of the model. Based on Table 5-2, the InP based type-II quantum well structure has a larger tunneling mass compared with other infrared photodiodes [104], which is desirable since the tunneling process will be suppressed at low bias. The larger tunneling mass may be due to the smaller wave function overlap in type-II structures. The trap energy level $\mathrm{E}_{\mathrm{t}}$ is close to the middle to effective band gap $(\sim 0.51 \mathrm{Eg})$ at temperatures from $200 \mathrm{~K}$ to $290 \mathrm{~K}$.

Based on Figure 5-7, we can conclude that the performance of lattice matched InGaAs/GaAsSb photodiodes is dominated by generation-recombination processes at small reverse biases (up to $5 \mathrm{~V}$ ) at temperature between $200 \mathrm{~K}$ and $290 \mathrm{~K}$. This bias range is the typical operation bias of those PIN photodiodes for infrared detection. The current in this range is significantly lower than the photodiodes using InAs/GaSb type-II QWs, whose tunneling component of dark current is substantial within this bias range [104]. Furthermore, at larger reverse biases (greater than 10V), trap-assisted and direct tunneling components of this type-II InGaAs/GaAsSb QWs photodiodes begin to dominate.

\subsubsection{Optical Characteristics}

The normal incident photo response of the photodiode was measured at temperatures between $200 \mathrm{~K}$ and $290 \mathrm{~K}$ with the method described in chapter 4 . The results are shown in Figure 5-8. As expected, the device has an optical response out to a longer wavelength than the device with lattice matched $5 \mathrm{~nm}$ InGaAs/ $5 \mathrm{~nm}$ GaAsSb quantum wells absorption [34, 79-81] and the response increases with temperature because the photo generated carriers have more thermal energy to transport over the hetero-barriers and 
transit to the electrodes. One unanswered questions is the mechanism by which these carriers get out of quantum wells, since both thermionic emission and phonon assisted tunneling can be the dominant carrier transport mechanism. This topic will be further discussed in Chapter 6.

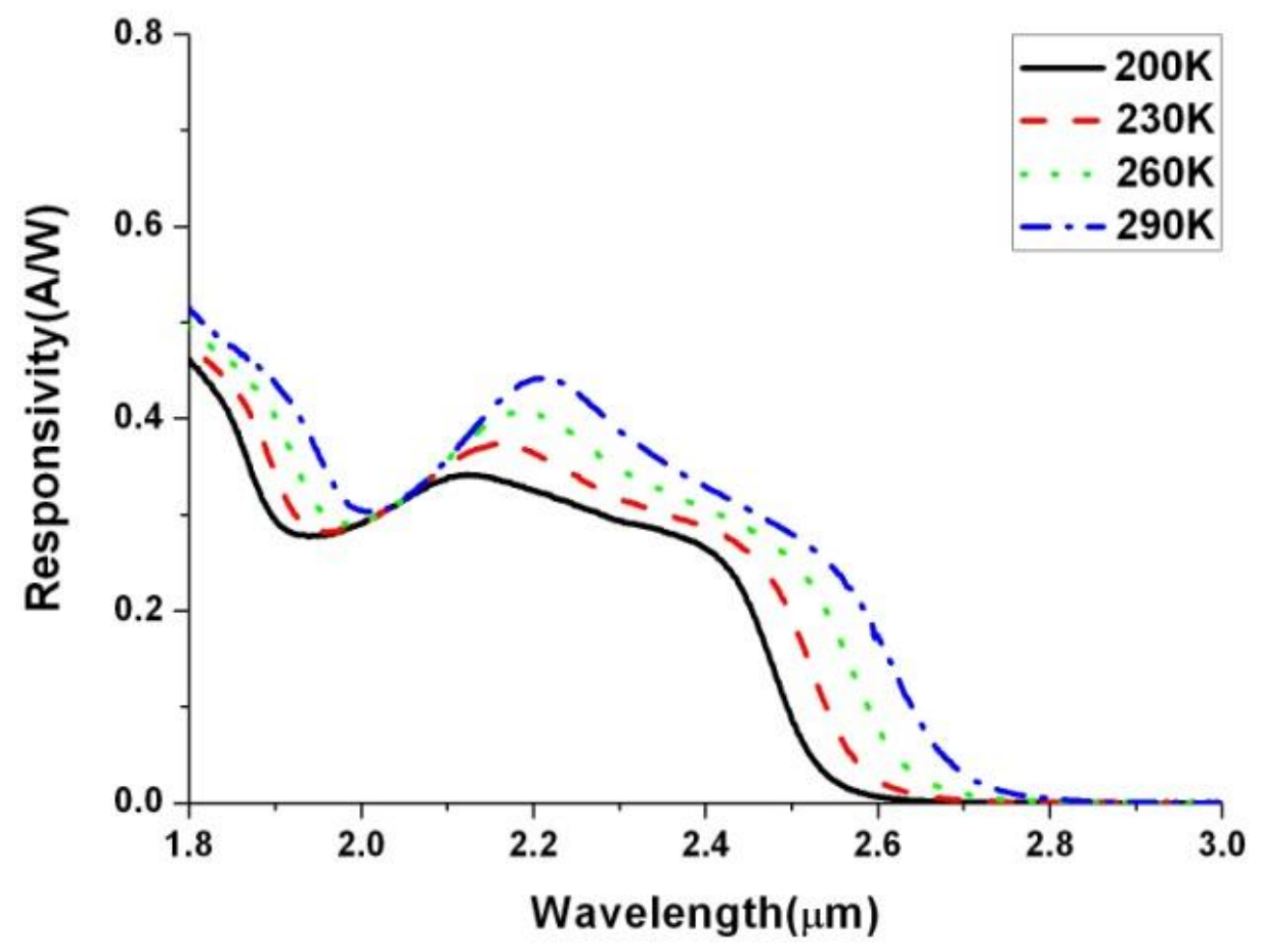

Figure 5.8 Normal incident photo response of the device under different temperatures, at reverse bias of $\mathbf{- 0 . 5 V}$ for Sample A

In order to investigate the responsivity under different biases and temperatures, the blackbody responsivity (BR) under different reverse biases and temperatures was measured and plotted in Figure 5-9. Here the blackbody responsivity is defined as the output photo current of device produced in response to a watt of input optical radiation 
from a blackbody source at $700{ }^{\circ} \mathrm{C}$. This blackbody response is proportional to the absolute value of responsivity, which can roughly represent the average optical response of the photodiodes.

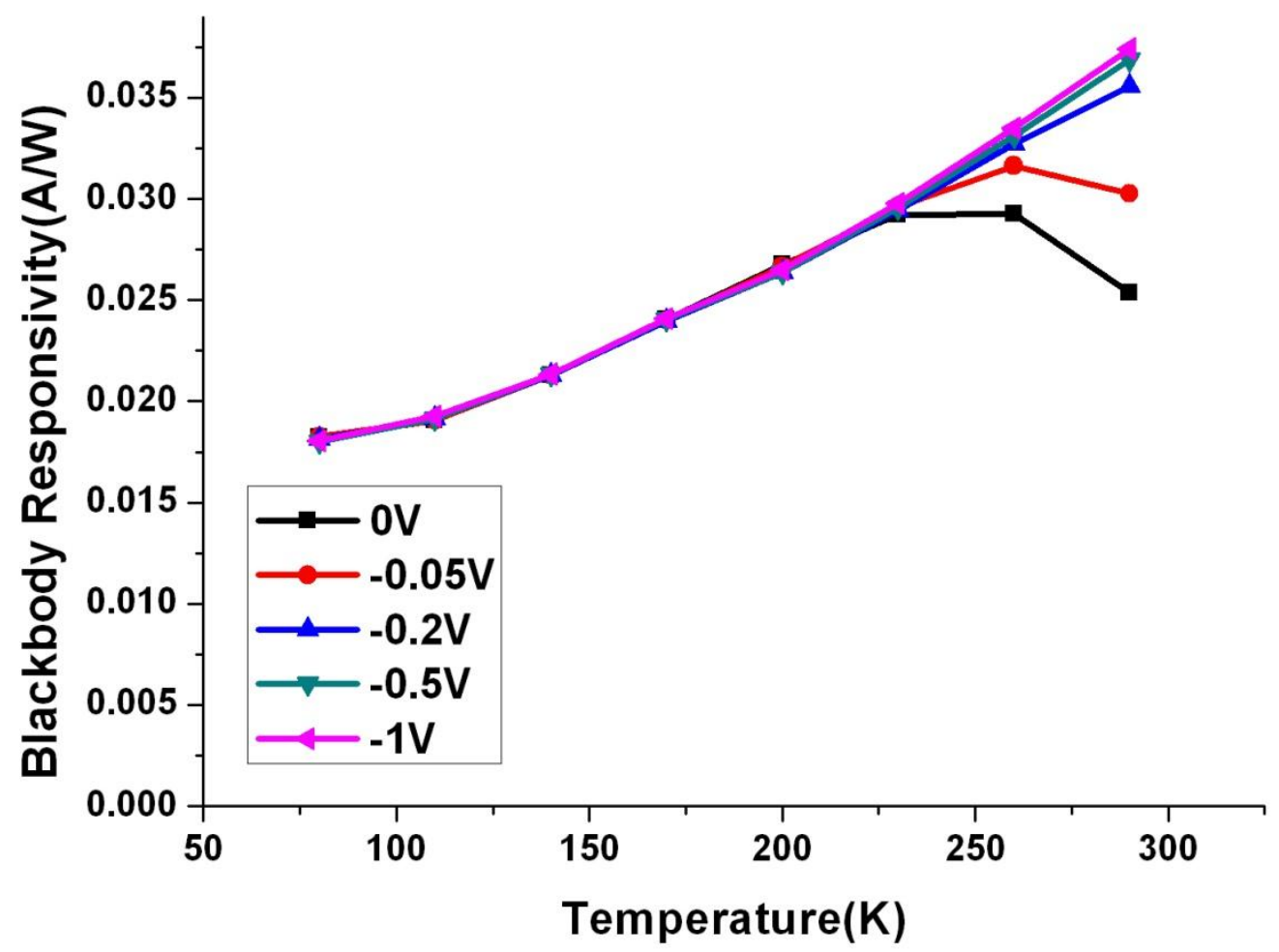

Figure 5.9 Blackbody responsivity versus temperature under different reverse bias for Sample A

For reverse biases above $0.2 \mathrm{~V}$, the blackbody responsivity for the device increases with temperature. However, the blackbody responsivity at smaller reverse biases initially increases with temperature and then decreases after some critical temperature. This critical temperature for Sample A is somewhere between $230 \mathrm{~K}$ and $260 \mathrm{~K}$ for reverse biases of $0 \mathrm{~V}$. The main cause of this effect is temperature and bias dependence of the thickness of depletion region in the absorption region. At higher temperatures, the absorption region is not fully depleted at small biases due to the unintentional doping 
concentration. As a result, the carriers generated outside the depletion region will recombine before they are collected. As the temperature decreases, the overall background doping concentration in absorption region drops due to incomplete dopant ionization, and thus, the absorption region becomes more depleted at small bias, which helps collect the photo generated carriers. In addition, the blackbody responsivity of the devices is almost independent of the reverse bias at temperatures below $230 \mathrm{~K}$. The reason is that at low temperatures, the absorption region is fully depleted even at small bias.

The device's specific detectivity (D*) as a function of wavelength is calculated from the responsivity and noise with the following formula [109]:

$$
\mathrm{D}^{*}=\frac{\mathrm{R} \sqrt{\mathrm{A}}}{\mathrm{S}_{\mathrm{n}}}
$$

where $\mathrm{R}$ is the responsivity, $\mathrm{A}$ is the diode area and $\mathrm{S}_{\mathrm{n}}$ is the noise spectrum density(see Figure 5.10). 


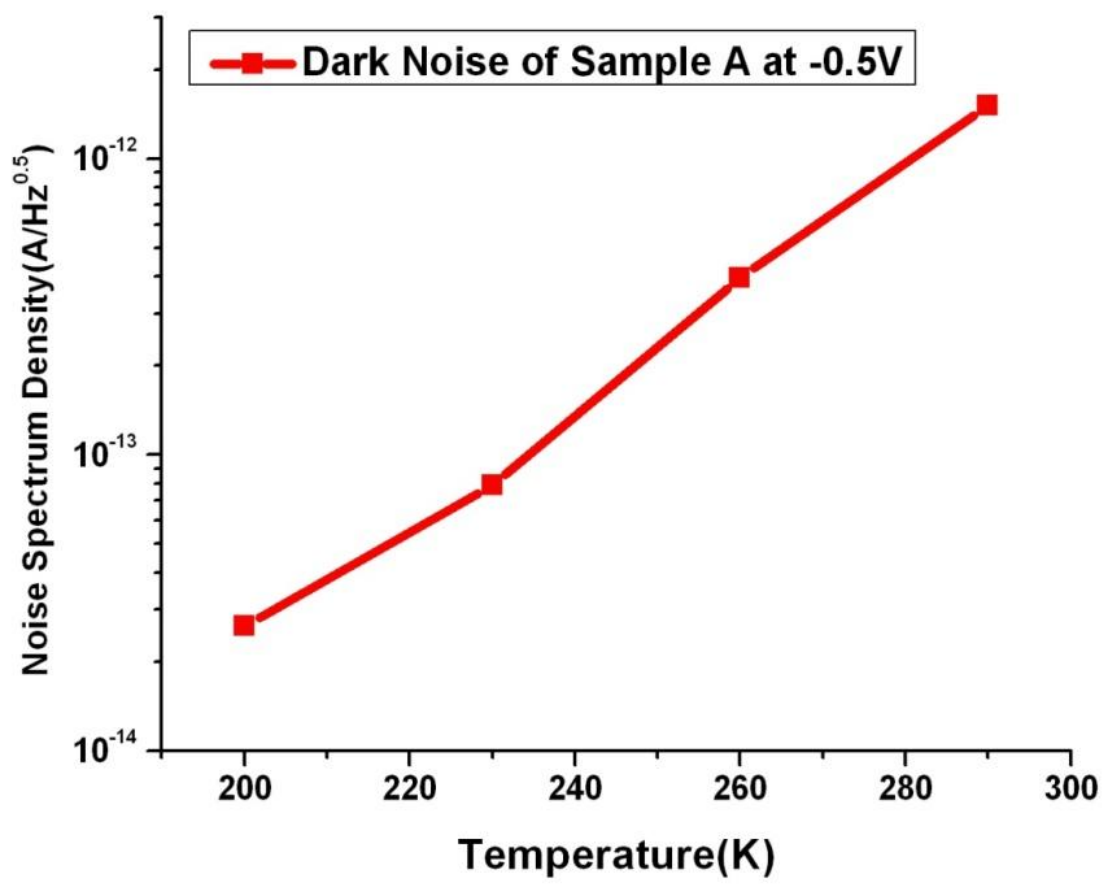

Figure 5.10 Dark noise spectrum densities versus temperature under bias of $\mathbf{- 0 . 5 V}$ for Sample A

The $\mathrm{D}^{*}$ versus wavelength for the devices at different temperatures is shown in Figure 5-11. D* for Sample A is $3.28 \times 10^{9} \mathrm{~cm} \cdot \mathrm{Hz}^{1 / 2} \cdot \mathrm{W}^{-1}$ at $\lambda=2.4 \mu \mathrm{m}$ at $290 \mathrm{~K}$ and increases to $1.51 \times 10^{11} \mathrm{~cm} \cdot \mathrm{Hz}^{1 / 2} \cdot \mathrm{W}^{-1}$ at $200 \mathrm{~K}$. These values are plotted in Figure 5-12 to allow a comparison with Sidhu's device [34] and other commercially available photodetectors. These results show that my devices have similar performance to the other approaches. In many cases, this performance is achieved at much higher operating temperatures. Due to better noise performance of Sample A at 200K, the D* performance at Sample A is higher compared to Sidhu's device. 


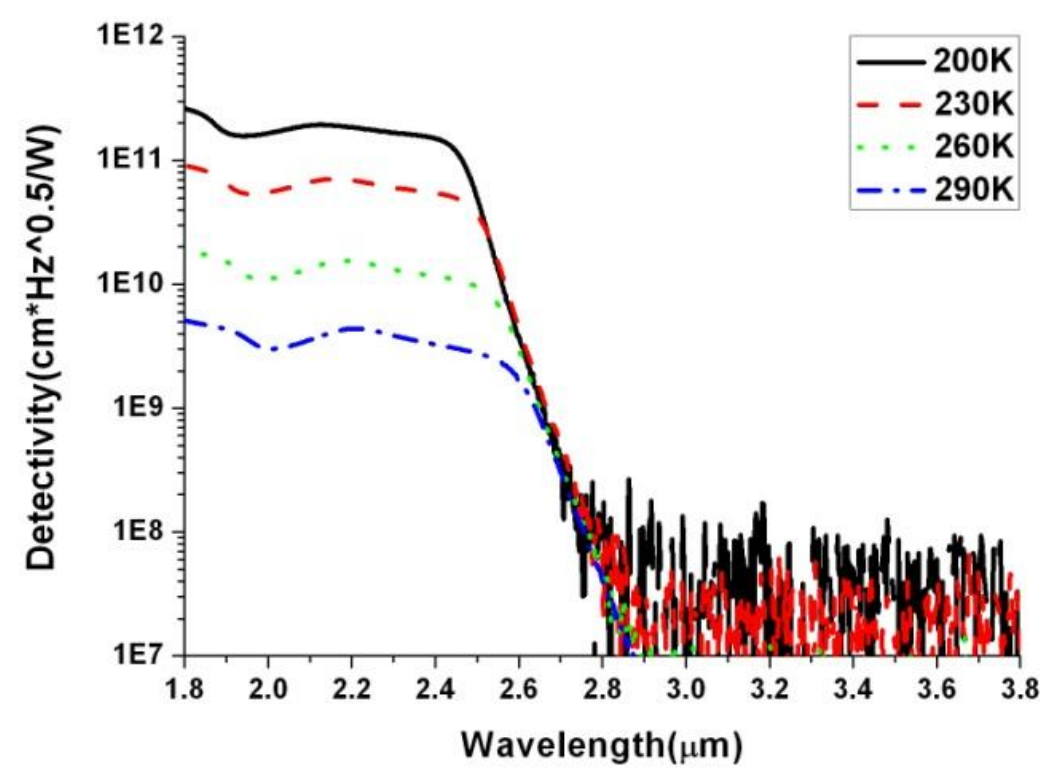

Figure 5.11 Detectivity of Sample A at different temperatures under reverse bias of $\mathbf{- 0 . 5 \mathrm { V }}$

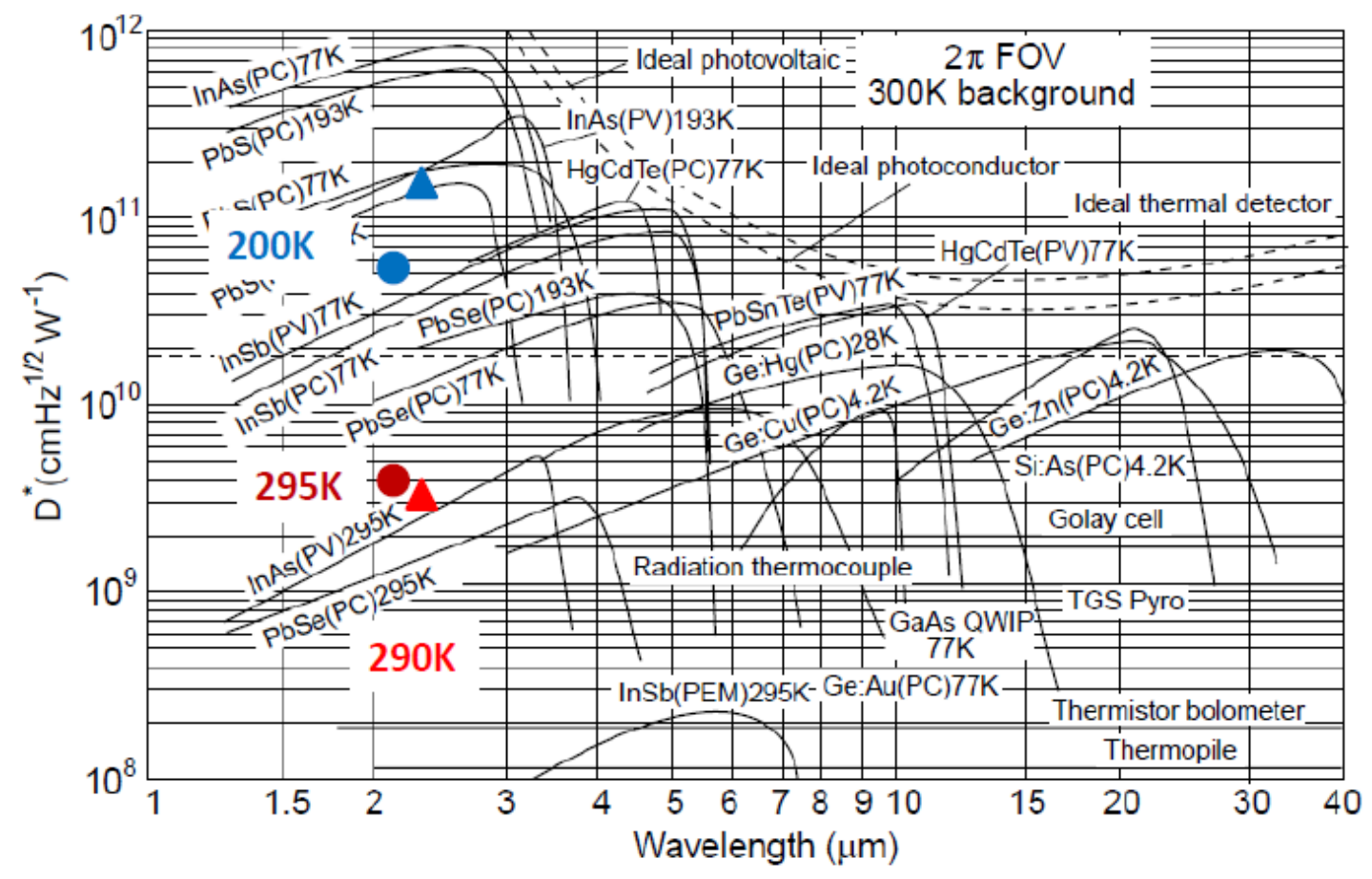

Figure 5.12 Comparison of the detectivities of Sample A at $2.4 \mu \mathrm{m}$ (triangle) with that of Sidhu's device (dot)[34] and other infrared detectors [36] 
For infrared imaging application, infrared radiation may not be normal incident. Therefore, it is also important to study the angular dependence of the optical response of these photodiodes. It was measured with blackbody radiation, by rotating the wire bonded sample in the chamber. The angular responsivity $R(\theta)$ of the device can be calculated from the relation

$$
R(\theta)=\mathrm{R}(0) \frac{\mathrm{I}(\theta)}{\mathrm{I}(0)} \frac{\mathrm{S}(0)}{\mathrm{S}(\theta)}
$$

where $\theta$ is the incident angle, $R(0)$ is the normal incident responsivity, $I(\theta)$ is the photo current measured at incident angles of $\theta, S(\theta)$ is the projected area of the detector on the normal plane of the light beam at angles $\theta$. Figure 5.14 shows the angular dependence of the normalized blackbody responsivity of the $350 \mu \mathrm{m}$ diameter photodiodes with incidence angles ranging of $0^{\circ}$ to $75^{\circ}$.

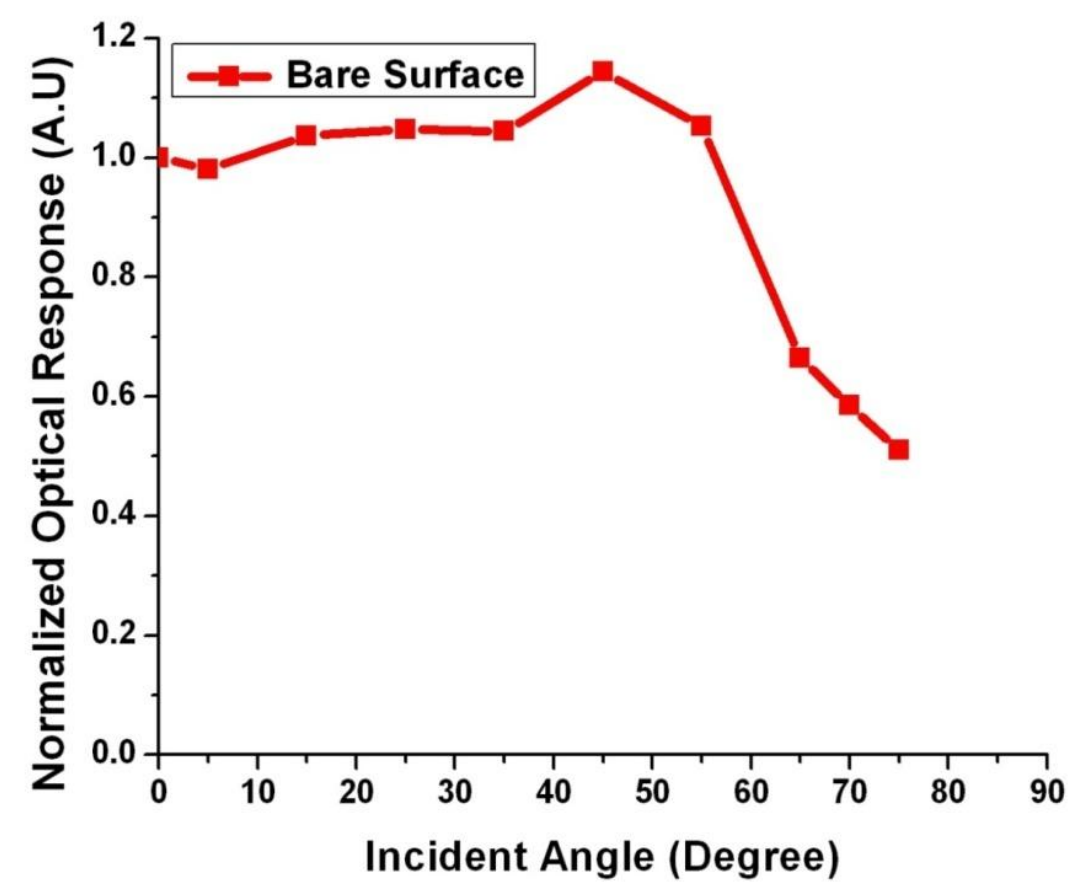

Figure 5.13Angular dependence of normalized blackbody responsivity of Sample A under zero bias 
Based on the Figure 5.14, for the incidence angles smaller than $55^{\circ}$, a slightly increase of responsivity was observed, which is due to the increase in optical absorption length. For the incidence angles larger than $55^{\circ}$, the responsivity begins to drop.

The bandwidth of Sample A is estimated by measuring the temporal response to 1550 $\mathrm{nm}$ optical pulse laser. The optical pulse width of the laser is approximately $46 \mathrm{fs}$ and the photodiodes is biased at $-0.1 \mathrm{~V}$. Figure 5.13 (a) shows the transient electrical signal with the pulse laser shining on the $100 \mu \mathrm{m}$ diameter photodiode. The full width at halfmaximum (FWHM) is around 0.5ns. Fourier transform of the transient response was carried out as shown in Figure 5.13(b). The 3-dB bandwidth can be estimated from Figure 5.13(b), which is around $565 \mathrm{MHz}$, and this bandwidth is RC-limited. 


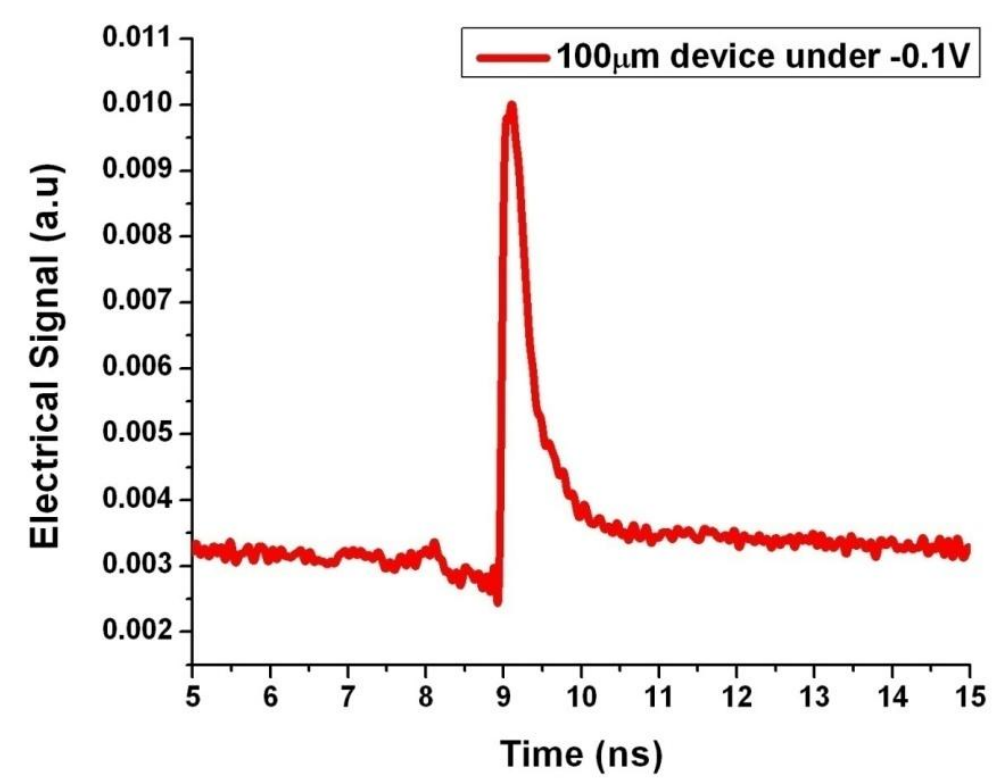

(a)

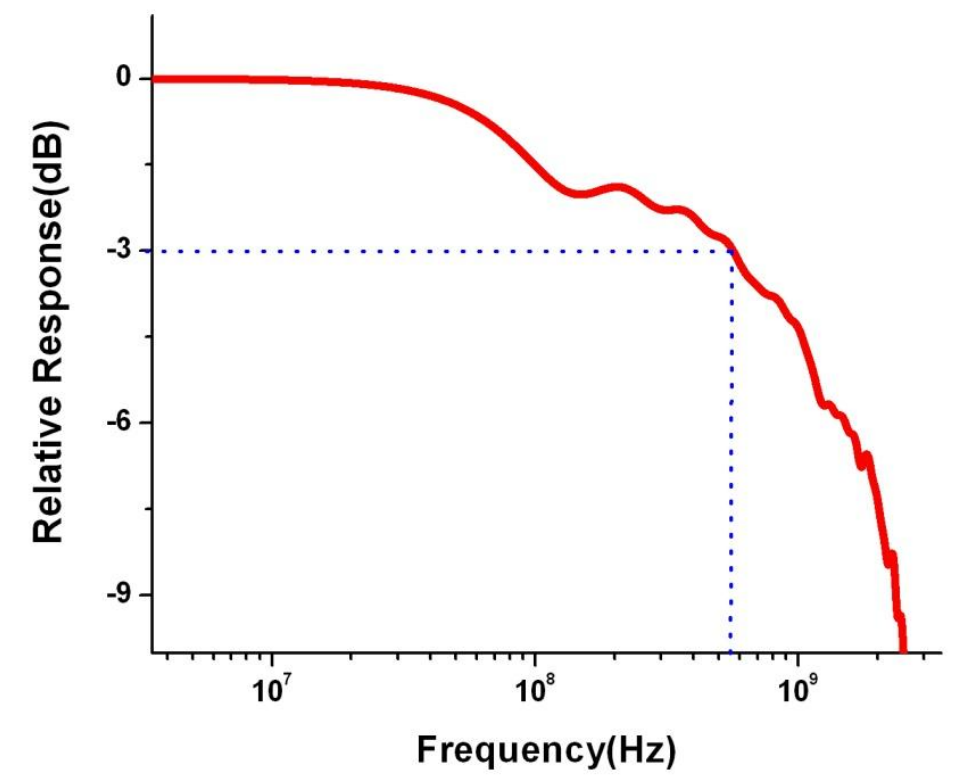

(b)

Figure 5.14 (a) Optical transient response of $100 \mu \mathrm{m}$ photodiode under bias of $\mathbf{- 0 . 1 V}$ (b) Fourier transform of the pulsed transient response of $100 \mu \mathrm{m}$ photodiode 


\subsection{Strain Compensated Quantum Wells Photodiode (Sample B)}

One of the advantages of InGaAs/GaAsSb quantum wells is that strain can be used to extend the detection wavelength. Sample B (see Figure 5-15) was designed to determine the effects of the extension of detection wavelength on other performance metrics. The transitions between the lowest energy states in InGaAs and GaAsSb are expected to occur around $0.38 \mathrm{eV}$, corresponding to transition wavelength of around $3.2 \mu \mathrm{m}$.

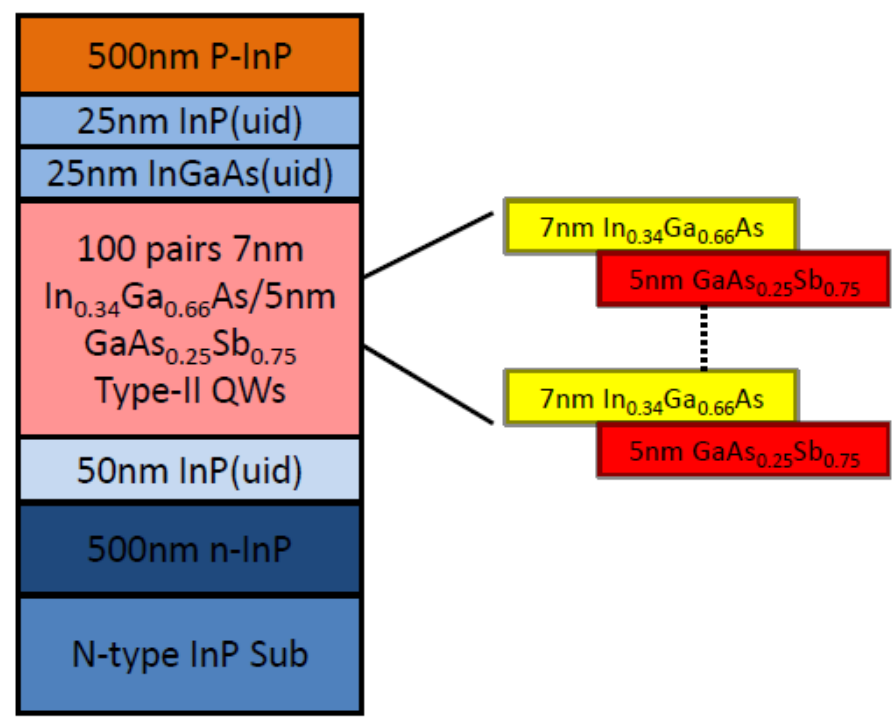

Figure 5.15 A schematic of the PIN device structure with type-II $7 \mathbf{n m ~} \operatorname{In}_{0.34} G a_{0.66} A s / 5 n m$ $\mathrm{GaAs}_{0.25} \mathrm{Sb}_{0.75} \mathrm{MQWs}$ as absorption layer

Material composition and thickness of layers are also verified using high resolution Xray diffraction (HRXRD) as shown in Figure 5-16. The appearances of a large number of satellite peaks suggest that the structure is of good crystal quality and have abrupt interfaces in the absorption region. One difference between samples A and B is the full- 
width at half-maximum (FWHM) of the satellite peaks. In Sample B they are larger than the simulation suggesting that some strain relaxation may be occurring.

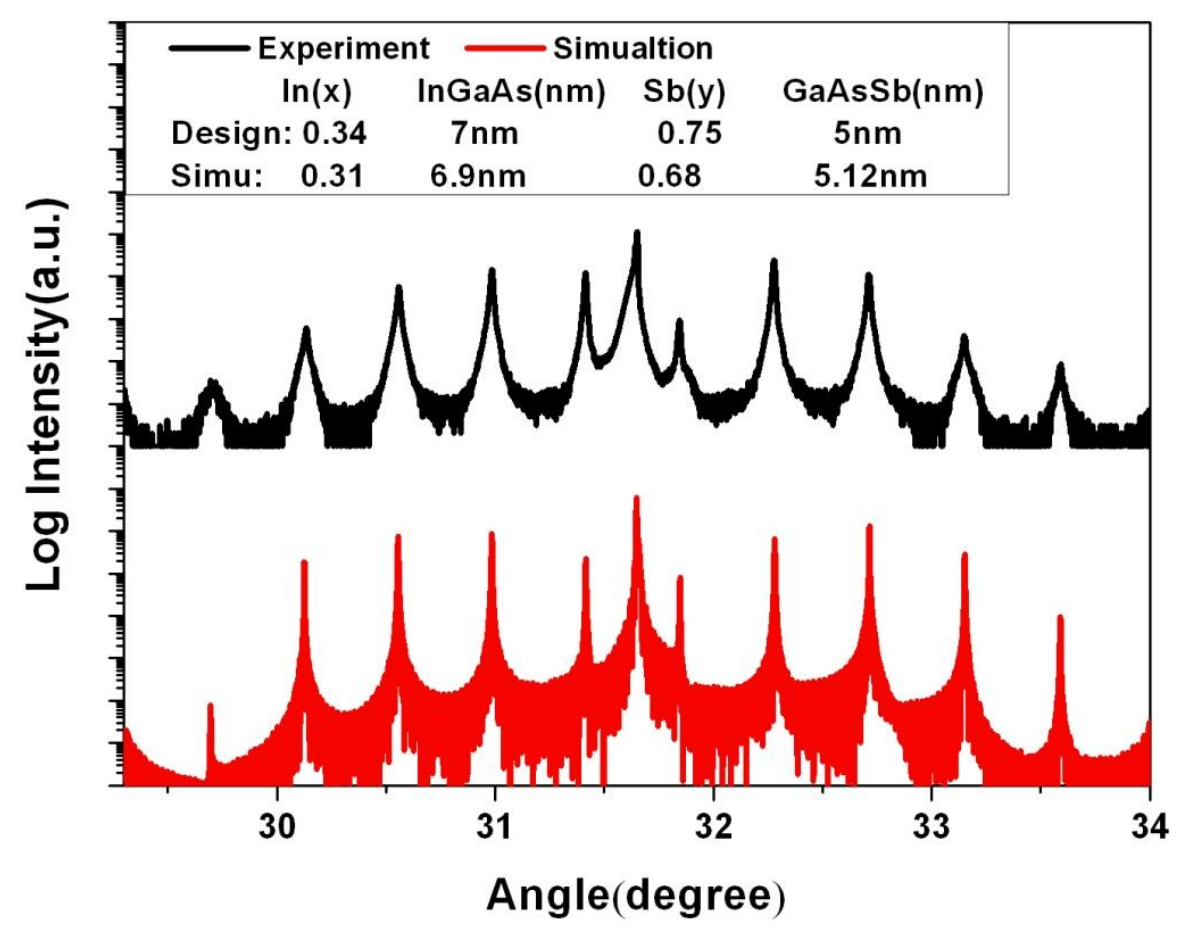

Figure 5.16 HRXRD data of Sample B of 100 pairs of $7 \mathrm{~nm} \mathrm{In}_{0.34} \mathrm{Ga}_{0.66} \mathrm{As} / 5 \mathrm{~nm} \mathrm{GaAs}_{0.25} \mathrm{Sb}_{0.75}$

\subsubsection{Characteristics}

The dark current-voltage (I-V) characteristics of Sample B were also measured using an HP4145B semiconductor parameter analyzer. Twenty-one out of thirty devices show very similar dark current with low variations. The dark current-voltage (I-V) characteristics at different temperatures for the device with diameter of $180 \mu \mathrm{m}$ are shown in Figure 5-17. The dark current density is $9.7 \mathrm{~mA} / \mathrm{cm}^{2}$ at $290 \mathrm{~K}$ at $-0.5 \mathrm{~V}$ and decreases to $58.6 \mu \mathrm{A} / \mathrm{cm}^{2}$ at $200 \mathrm{~K}$. The dark current of the Sample B is higher than that of Sample A, which is due to the fact that there is higher thermal generation-recombination rate in the 
absorption region, since Sample B has the smaller effective band gap and higher defect density compared to Sample A.

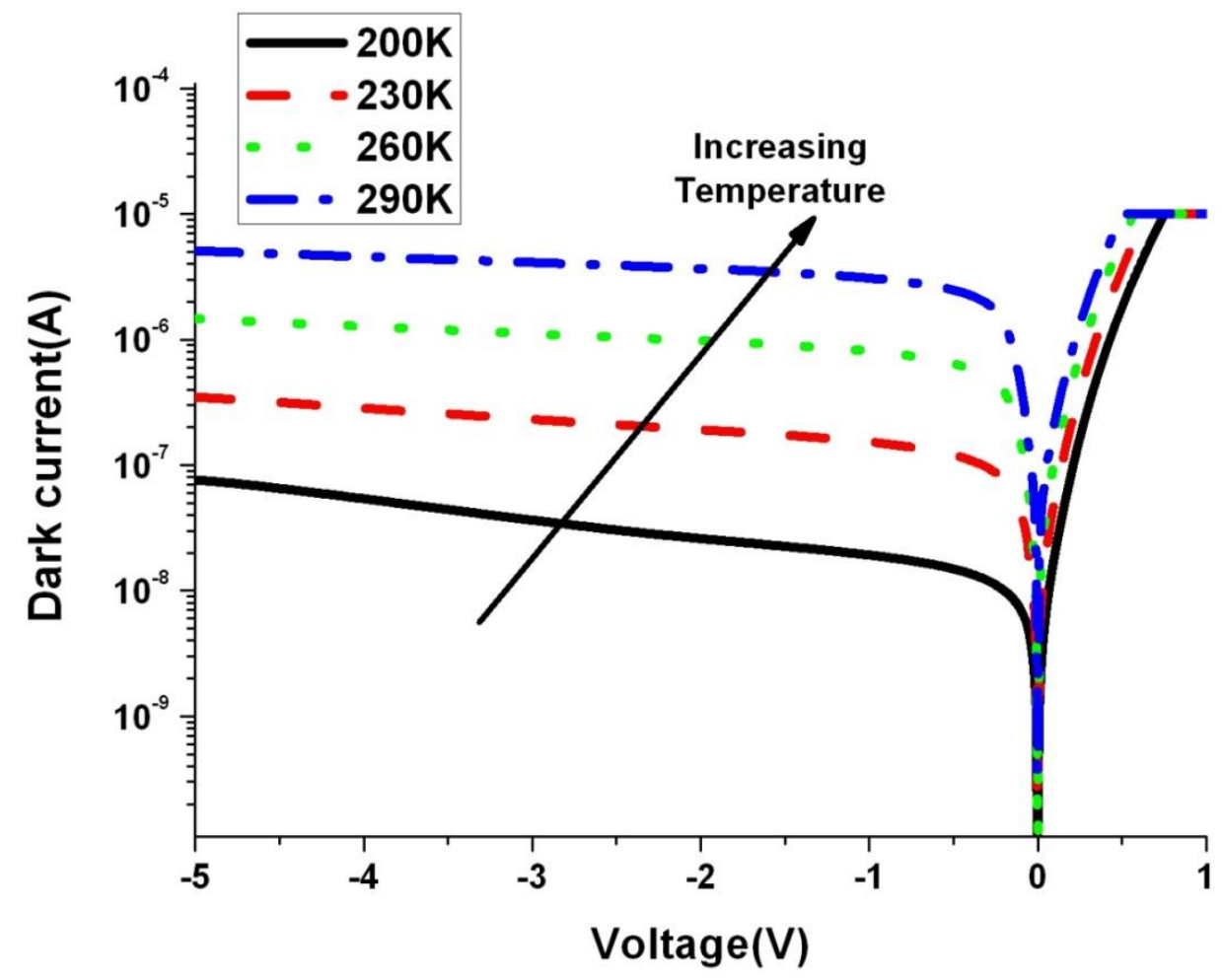

Figure 5.17 Dark current versus voltage for a $180 \mu \mathrm{m}$ diameter device measured at different temperatures

The dark current-voltage (I-V) characteristics of Sample B at 290K with different device area are shown in Figure 5-18. The dark current of the Sample B scales with device area which indicates the dark current is dominated by the bulk component as was the case with Sample A.

The Arrhenius plot of the dark current for Sample B is also shown in Figure 5-19. The activation energy is around $0.28 \mathrm{eV}$ for this device. The activation energy difference 
between Samples A and B is due to the different effective band gap and different properties of the traps in the two samples.

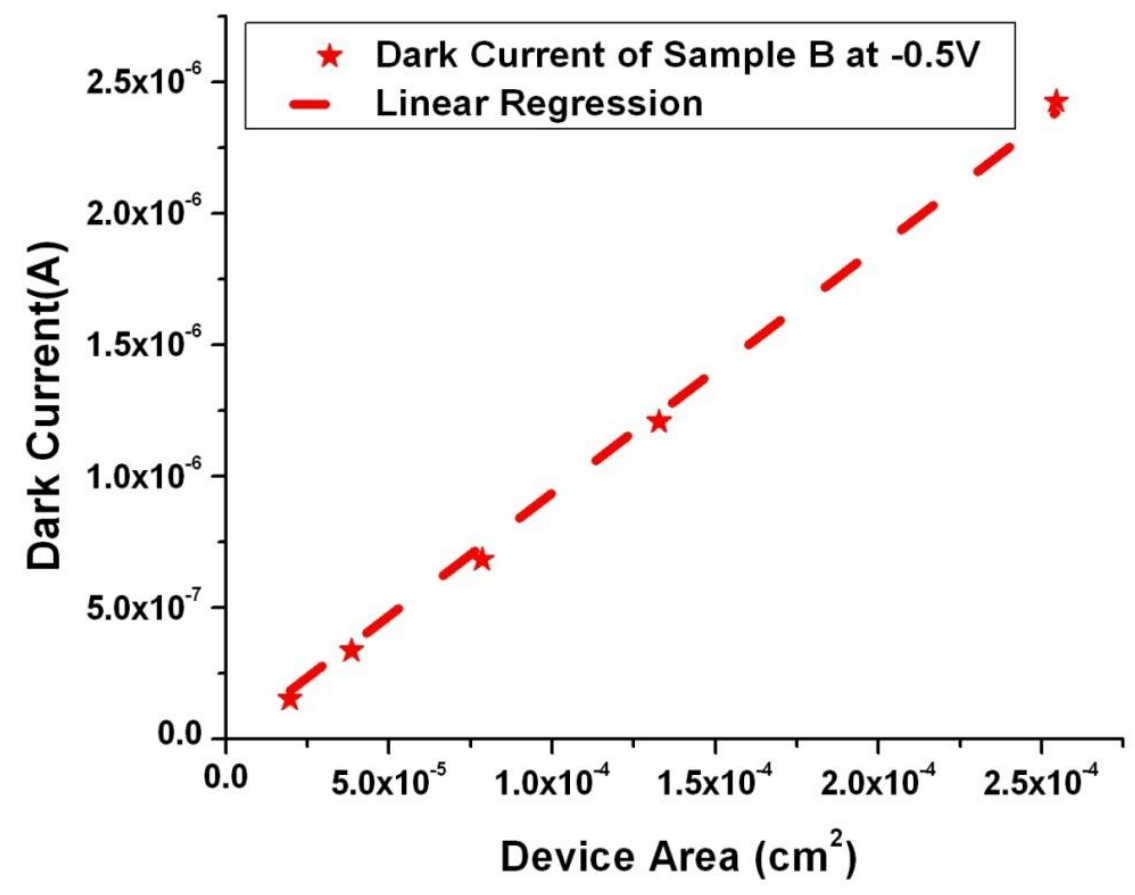

Figure 5.18 Dark current versus device area for lattice matched Sample B with bias of $\mathbf{- 0 . 5 \mathrm { V }}$ measured at $290 \mathrm{~K}$ 


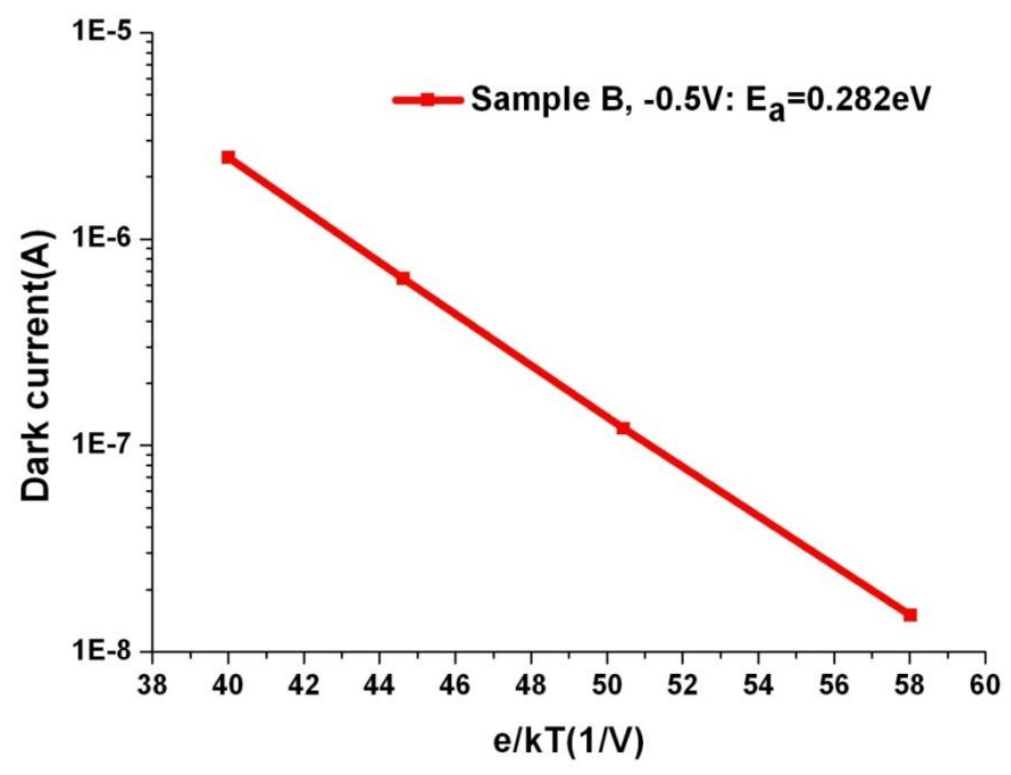

Figure 5.19 Arrhenius plot of the dark current for $180 \mu \mathrm{m}$ diameter Device $B$ at reverse bias $-\mathbf{0 . 5 V}$

In addition, the $\mathrm{R}_{0} \mathrm{~A}$ product for Sample $\mathrm{B}$ at different temperatures is shown in Figure 5-20 compared to that of Sample A. The $\mathrm{R}_{0} \mathrm{~A}$ at $290 \mathrm{~K}$ is slight larger than that of Sample A, but at $200 \mathrm{~K}$, it is approximately one order of magnitude lower than that of Sample A. This difference can be explained as follows. At 290K, Sample B is less depleted at zero bias due to the higher background unintentional doping concentration in the absorption region compared to that of Sample A, which will be confirmed in next section. Therefore, generation-combination current is smaller due to the thinner depletion region near zero bias in Sample B. As a result, at 290K, Sample B has slight larger $\mathrm{R}_{0}$ A product. At 200K, the $\mathrm{R}_{0} \mathrm{~A}$ product of Sample $\mathrm{B}$ is lower than Sample B, which is due to the smaller effective band gap and higher defect density in the absorption region of Sample B. 


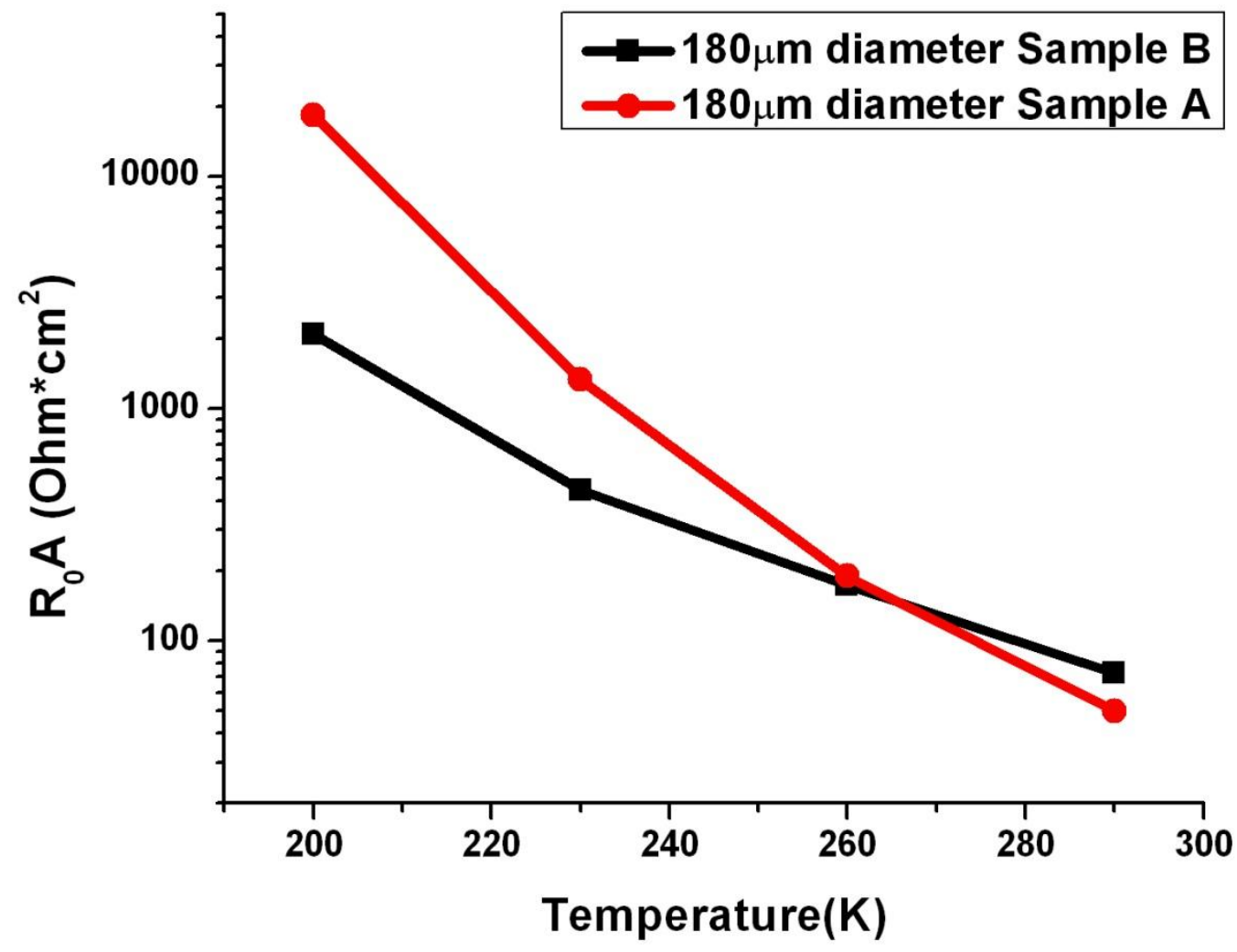

Figure $5.20 R_{0} A$ for Sample $B$ and Sample A with $180 \mu \mathrm{m}$ diameter at different temperatures

\subsubsection{Optical Characteristics}

The normal incident photo response of Sample B was measured at temperatures between $200 \mathrm{~K}$ and $290 \mathrm{~K}$. The results are shown in Figure 5-21. The device has an optical response out to wavelength of $3.2 \mu \mathrm{m}$ at $290 \mathrm{~K}$, which is consistent to what we expect based on our initial design. The response also increases with temperature as in Sample A. This is the first photodiode demonstrated on InP substrate which has optical response beyond $3.0 \mu \mathrm{m}$ using the interband absorption without using lattice mismatch layers. 


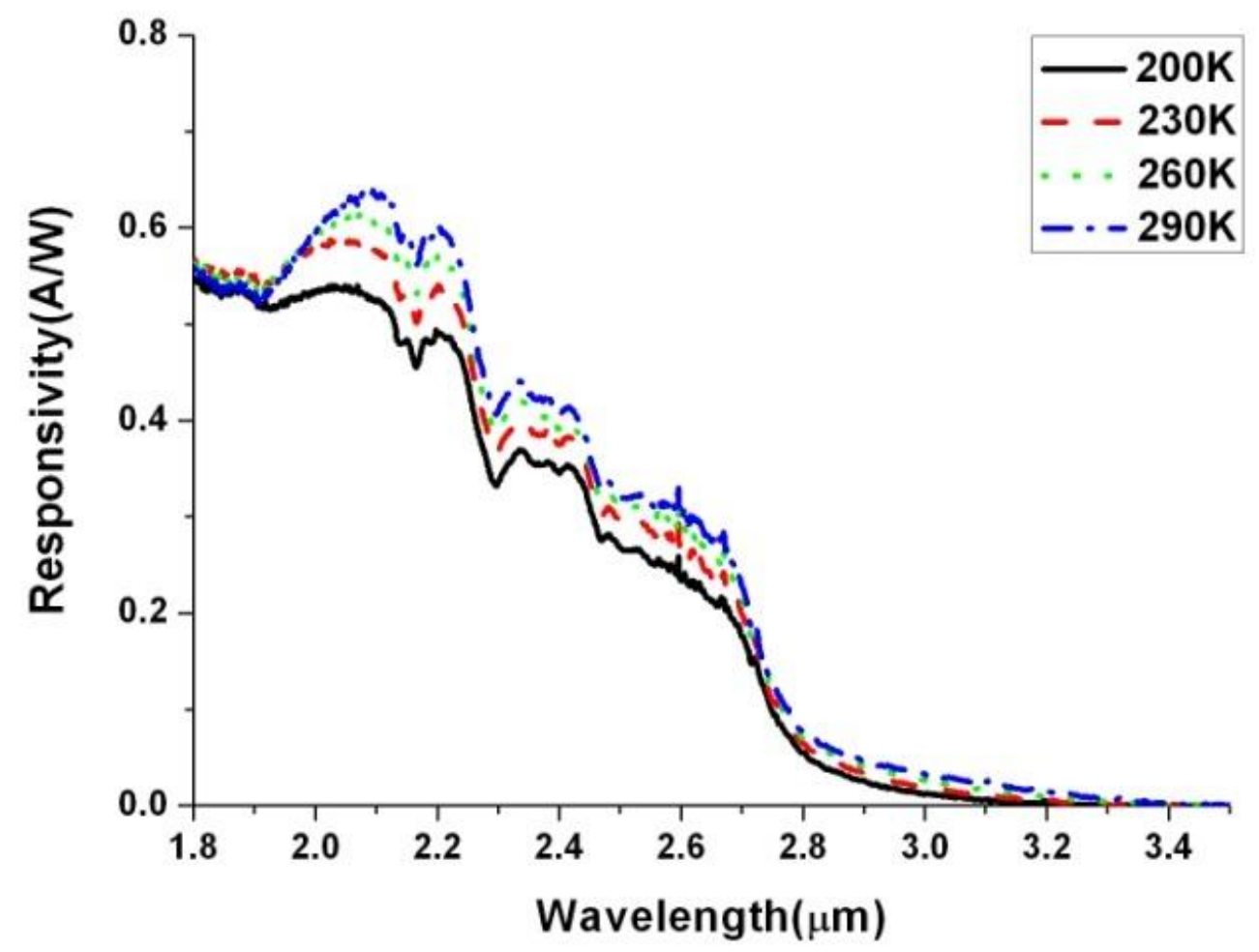

Figure 5.21 Normal incident photo response of the device under different temperatures, at reverse bias of $-\mathbf{0 . 5 V}$ for Sample $\mathrm{B}$

The blackbody responsivity of Sample B is shown in Figure 5-22. For reverse biases above $0.5 \mathrm{~V}$, the blackbody responsivity increases with temperature as seen in Sample A. Similarly, the blackbody responsivity of Sample B at small reverse biases initially increases with temperature and then decreases after some critical temperature. This critical temperature for Sample B is $140 \mathrm{~K}$ and $230 \mathrm{~K}$ for reverse biases of $0 \mathrm{~V}$ and $-0.2 \mathrm{~V}$ respectively. The main cause of this effect is similar to Sample A: temperature and bias dependence of the thickness of depletion region in the absorption region. At high temperatures and small biases, the absorption region is not fully depleted due to the unintentional doping concentration, and the carriers generated outside the depletion 
region have to diffuse into the depletion region before getting collected. Therefore, the carrier collection efficiency is relatively low, and the responsivity is small. As the temperature decreases, the overall background doping concentration in absorption region drops due to incomplete dopant ionization, thus, the absorption region becomes more depleted at small biases, which helps collect the photo generated carriers. The lower critical transition temperature of Sample B compared to Sample A is due to a higher background doping concentration in absorption region for Sample B. This was confirmed by Capacitance-Voltage (C-V) measurements (see Figure 5-23). The larger capacitance of Sample B at small bias compared to Sample A indicates that the absorption region of Sample B has a higher background doping. The background doping can be extracted from the linear regression of $1 / \mathrm{C}^{2}-\mathrm{V}$ plot [107], which is estimated to be around $4 \times 10^{15} \mathrm{~cm}^{-3}$ and $1 \times 10^{15} \mathrm{~cm}^{-3}$ for Sample B and Sample A, respectively. Thus Sample B needs a higher bias in order to fully deplete the absorption region.

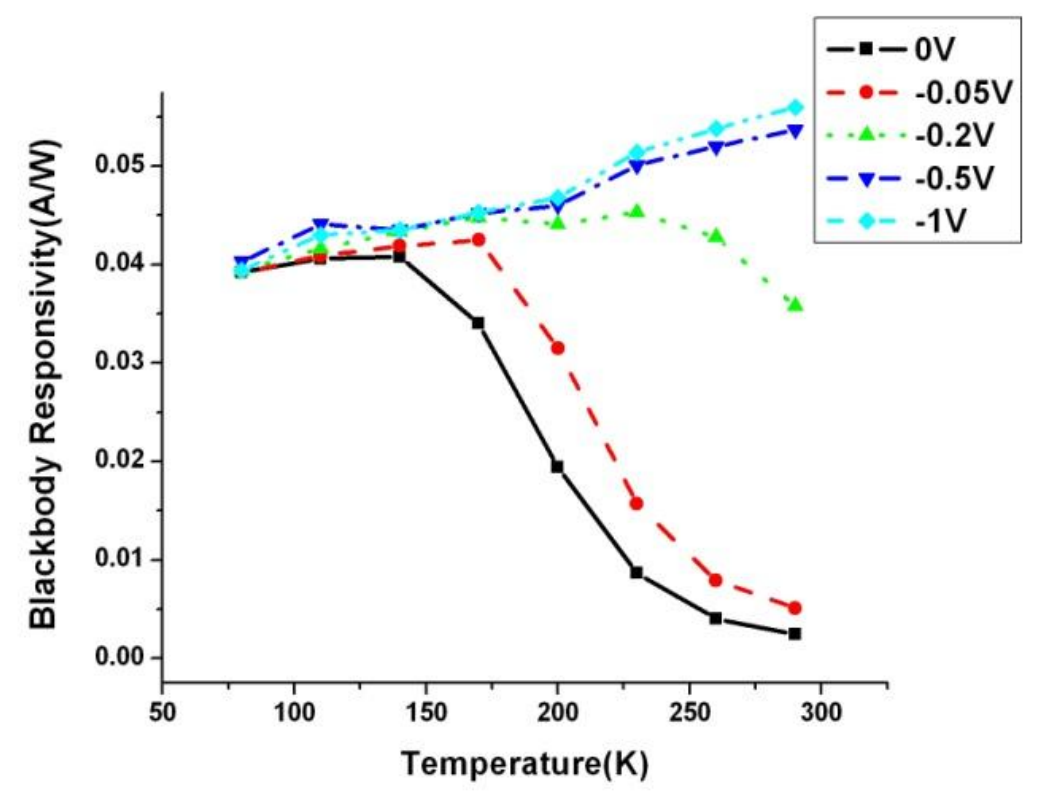

Figure 5.22 Blackbody responsivity versus temperature under different reverse bias for Sample B 


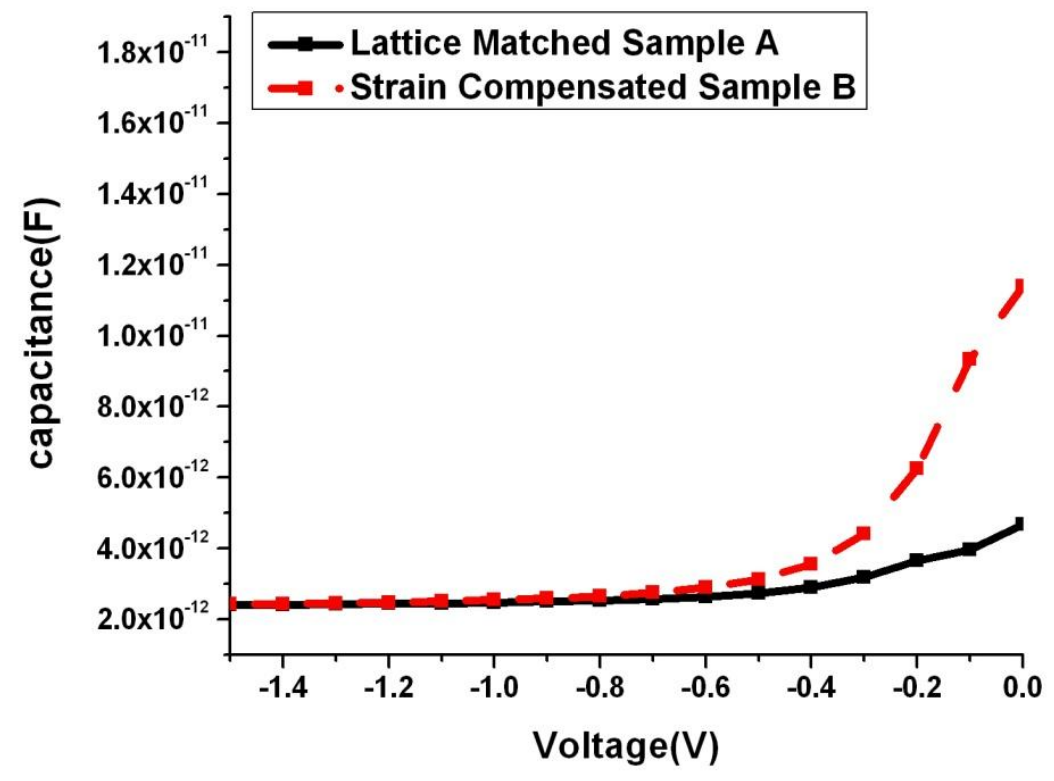

Figure 5.23 Capacitance-Voltage (C-V) measurements of the $180 \mu \mathrm{m}$ diameter devices for both Sample A and Sample B

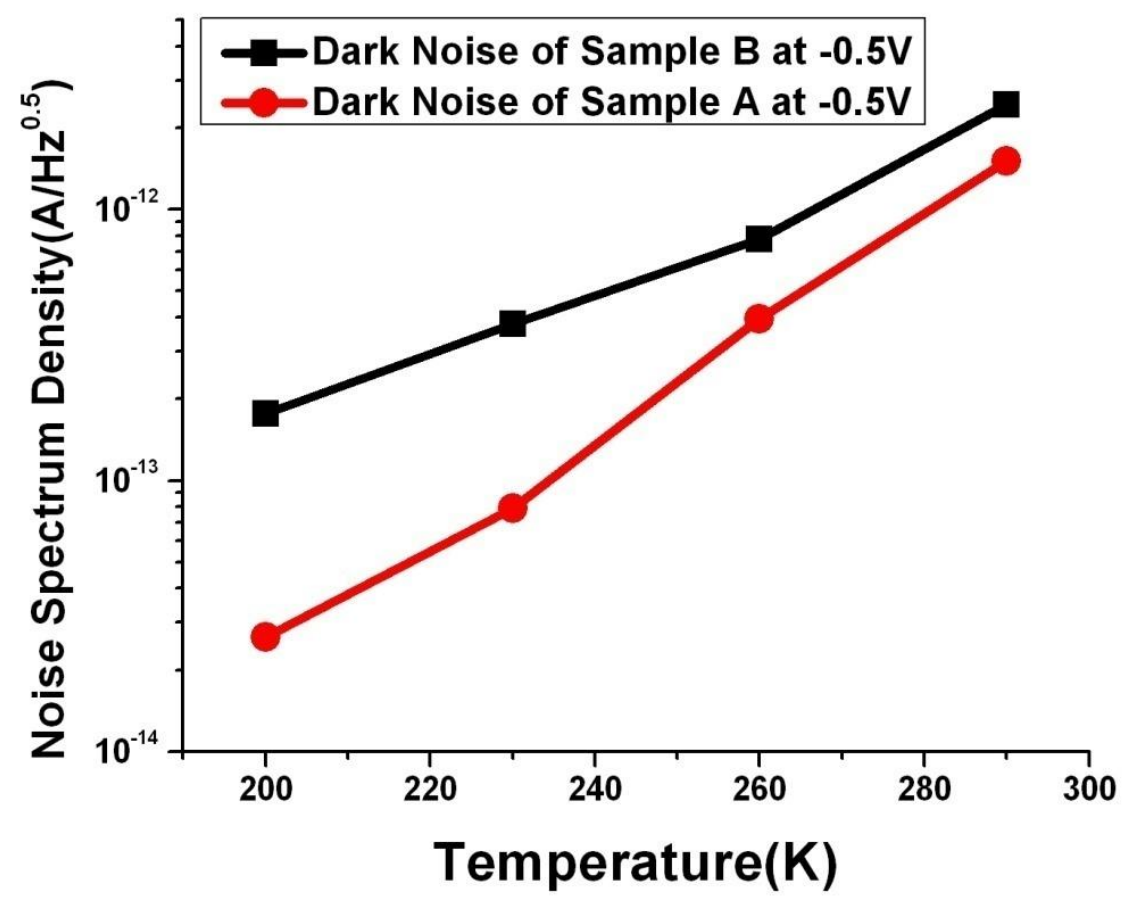

Figure 5.24 Dark noise spectrum densities versus temperature under reverse bias of $0.5 \mathrm{~V}$ for Sample B and Sample A 
The dark noise spectrum density of Sample B versus temperature was also measured as shown in Figure 5-24. Compared with Figure 5-10, the noise of Sample B is larger than Sample A at $290 \mathrm{~K}$ by a factor of two; this difference increases as the temperature decreases. This phenomenon is consistent to the comparison of dark current, $R_{0} A$ product between two samples, which is also due to the smaller effective band gap and higher defect density in the absorption region of Sample B. Furthermore, the $\mathrm{D}^{*}$ versus wavelength of the device at different temperatures is shown in Figure 5-25.

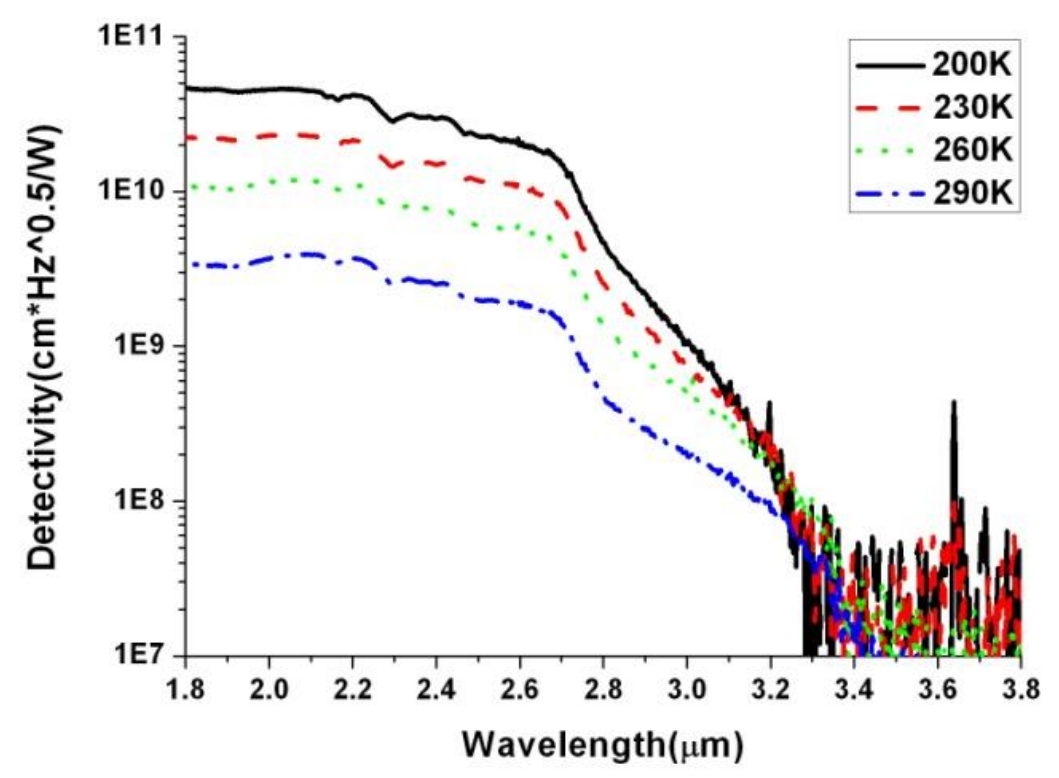

Figure 5.25 Detectivity of Sample B at different temperatures under reverse bias of $\mathbf{- 0 . 5 \mathrm { V }}$

As we can see from Figure $5-25$, for $\lambda=2.7 \mu \mathrm{m}$, D* for Sample B is $1.4 \times 10^{9}$ $\mathrm{cm} \cdot \mathrm{Hz}^{1 / 2} \cdot \mathrm{W}^{-1}$ at $290 \mathrm{~K}$ and increases to $1.5 \times 10^{10} \mathrm{~cm} \cdot \mathrm{Hz}^{1 / 2} \cdot \mathrm{W}^{-1}$ at $200 \mathrm{~K}$. For $\lambda=3.0 \mu \mathrm{m}, \mathrm{D}^{*}$ for Sample B is $2.0 \times 10^{8} \mathrm{~cm} \cdot \mathrm{Hz}^{1 / 2} \cdot \mathrm{W}^{-1}$ at $290 \mathrm{~K}$ and increases to $1.0 \times 10^{9} \mathrm{~cm} \cdot \mathrm{Hz}^{1 / 2} \cdot \mathrm{W}^{-1}$ at 200K. These values are also plotted in Figure 5-26 to compare to other InP based type-II 
quantum well photodiodes and other commercially available photodetectors. The performance is comparable with that of other commercially available photodetectors. Compared to the lattice matched Sample A, the D* performance of Sample B is inferior because of the higher defect density in the absorption region. Since strain compensated quantum wells can be grown on InP substrate without lattice mismatch layers, the defects in the quantum wells can be further eliminated with optimized growth, and better performance would be expected.

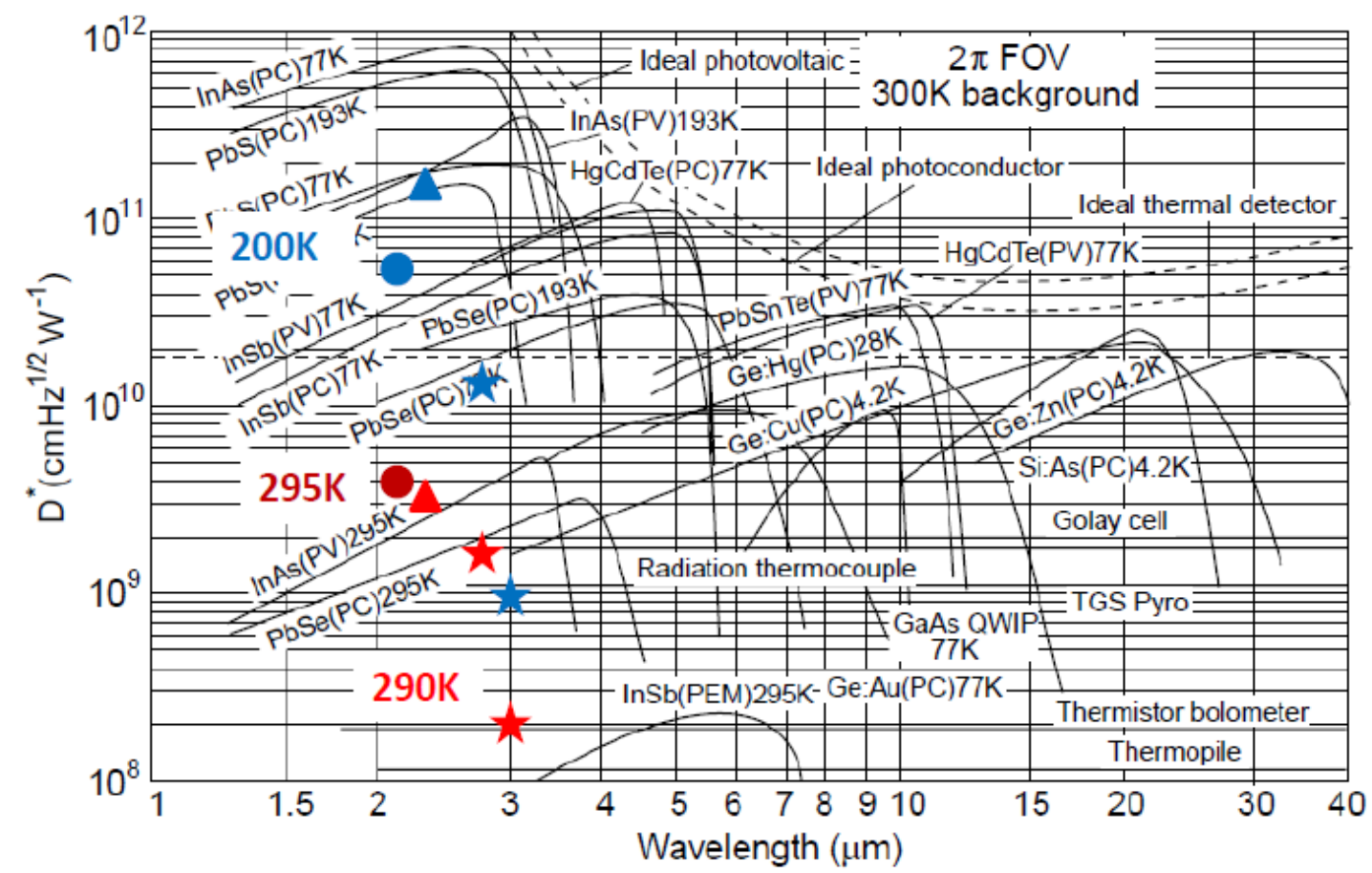

Figure 5.26 Comparison of the detectivities of Sample B at $2.7 \mu \mathrm{m}$ and $3 \mu \mathrm{m}$ (star) with that of Sidhu's

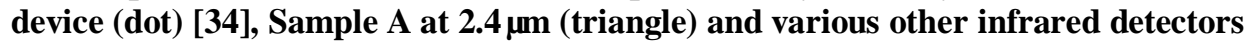

To understand the noise performance limitations of Sample B, the total noise current spectrum densities at room temperature are measured as a function of reverse bias as shown in Figure 5-27. Also for a direct comparison, the sum of the thermal noise and shot noise calculated based on equation (5.6) and (5.7) is also shown in Figure 5-27. 


$$
\begin{gathered}
i_{\text {thermal }}^{2}=\frac{4 k T \Delta f}{R} \\
i_{\text {shot }}^{2}=2 e I \Delta f
\end{gathered}
$$

As shown in Figure 5-27, the total measure noise is larger than the sum of thermal noise and shot noise. Moreover, the gap between each other increases significantly as reverse bias increases. This phenomenon indicates there are some other noise sources such as generation-recombination noise and 1/f noise contributing the total noise of the photodiode, which may be related to defects in the absorption region.

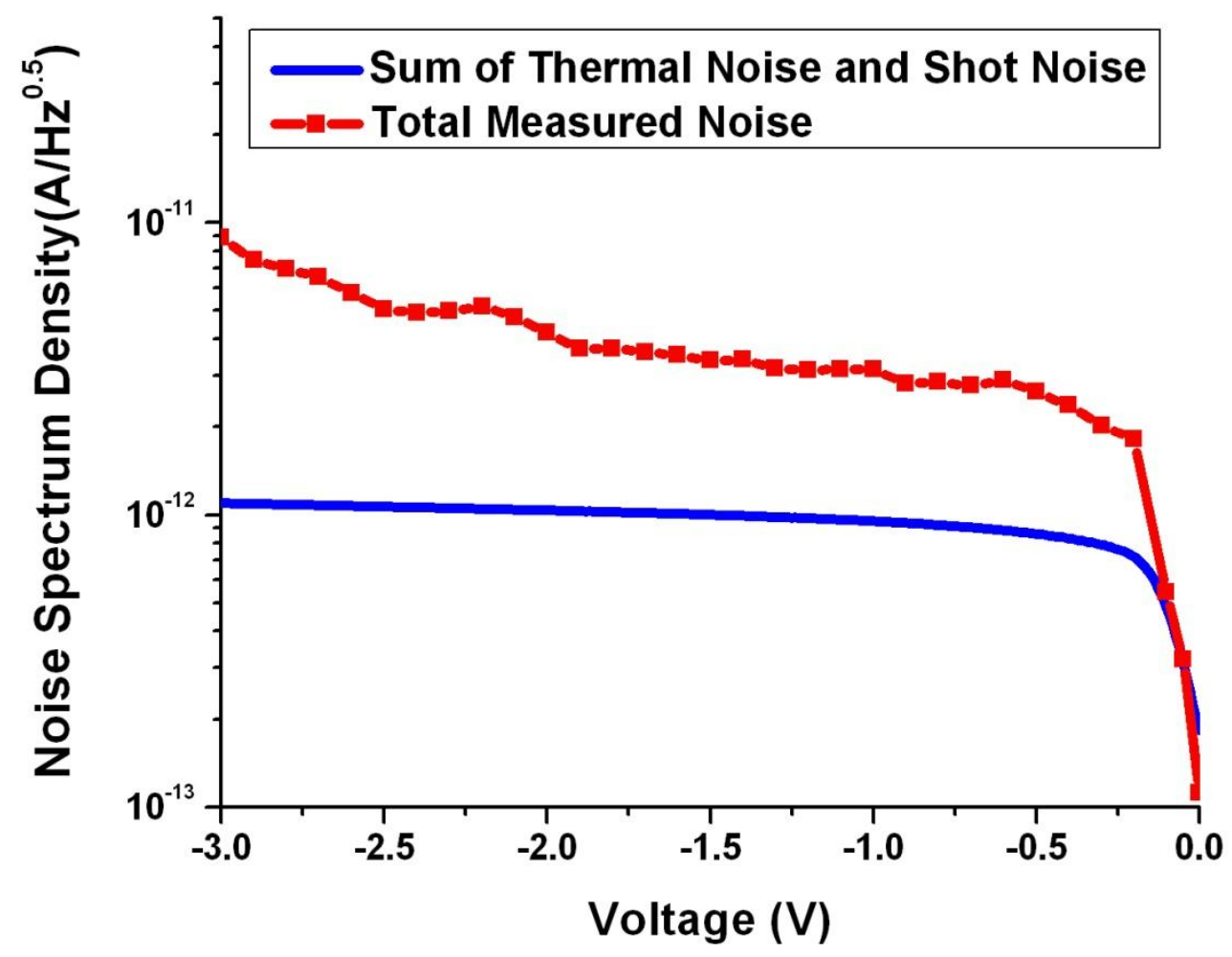

Figure 5.27 Noise versus reverse bias for a 180 diameter Sample B measured at room temperature

The blackbody responsivity (BR) and blackbody detectivity (BD*) versus the reverse bias are shown in Figure 5-28, in which the blackbody detectivity is defined as: 


$$
\mathrm{BD}^{*}=\frac{\mathrm{BR} \sqrt{\mathrm{A}}}{\mathrm{S}_{\mathrm{n}}}
$$

In Figure 5-28, blackbody responsivity increases positively with reverse bias. However, responsivity will saturate when the reverse bias is higher than $-0.5 \mathrm{~V}$, since the total number of e-h pair generated is roughly constant regardless of increase in the reverse bias. Furthermore, Figure 5-28 demonstrates that the device achieves optimum performance with a reverse bias around $-0.5 \mathrm{~V}$. If the voltage is increased, the noise of the device increases more rapidly than responsivity increases for the device. For lower biases, the optical response drops faster than the reduction in the device noise.

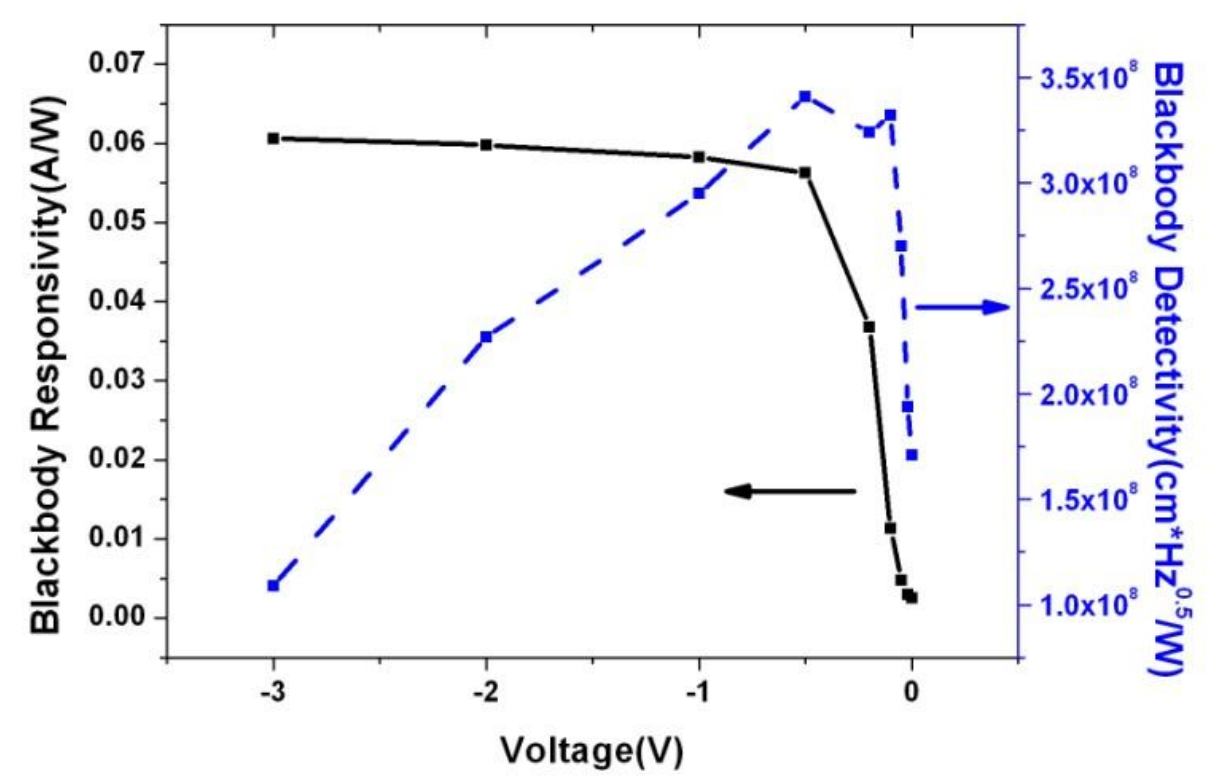

Figure 5.28 Blackbody responsivity and blackbody detectivity of Sample B versus reverse bias for a 180 diameter device measured at room temperature

If the operation temperature of the Sample B is reduced to $200 \mathrm{~K}$, the optimized reverse bias decreases to $0.2 \mathrm{~V}$ because of the smaller bias required to deplete the 
absorption region. Similar phenomena happened to Sample A. But the optimized operation reverse bias of Sample A at room temperature is around $-0.05 \mathrm{~V}$ (not $-0.5 \mathrm{~V}$ ), since Sample A have lower back ground doping concentration and better crystal quality in the absorption region.

\subsection{New Strain Compensated Quantum Wells Photodiode (Sample C)}

\subsubsection{Introduction and Device Design}

As indicated in Figure 3-12, even though longer wavelengths can be achieved by using thick InGaAs layers and very high Sb composition in the GaAsSb layer, the light absorption is weak due to a small wave function overlap between electron and hole. Therefore, there are some limitations by using the conventional strain compensated MQWs. In this section, the design and performance of a new strain compensated type-II quantum wells photodiode will be discussed, which has some potential advantages over the conventional strain compensated MQWs. 


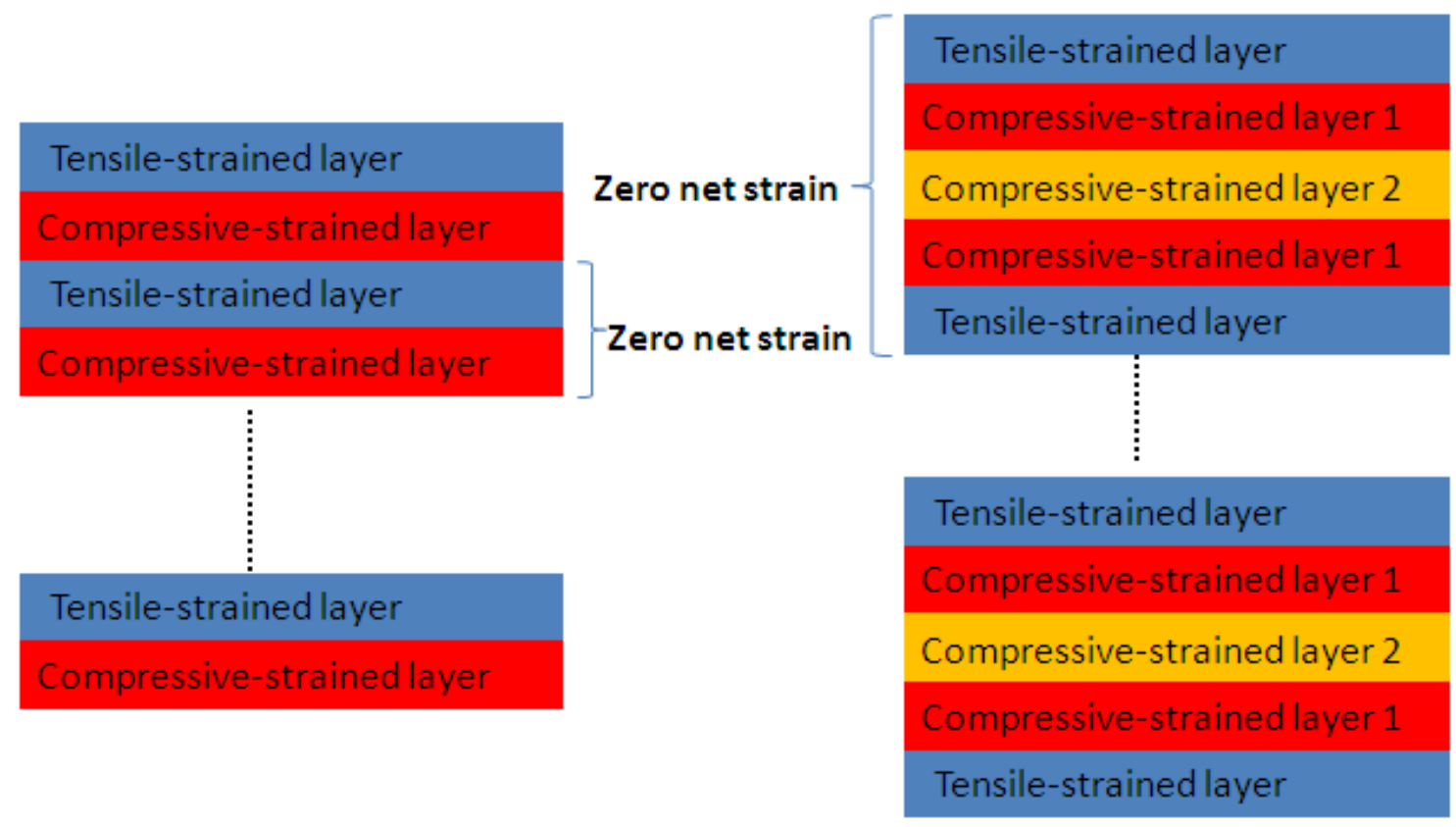

(a)

(b)

Figure 5.29 (a) the structure of conventional strain compensated MQWs, (b) the structure of new strain compensated MQWs

The difference between this new strain compensated MQWs structure and the previous conventional strain compensated MQWs structure is shown in Figure 5-29. Compared with the conventional strain compensated quantum wells structure, strain compensation is achieved by incorporating a tensile strained barrier layer between a set of compressively strained quantum wells. And the total strain of type-II quantum wells and the barrier layer is also set to zero. This enables the growth of thick absorption regions. In the conventional strain compensated MQWs, the spatially-indirect type-II transition occurs between the conduction band states in tensile strained layer and valence band states in the compressively strained layer, while in the new strain compensated MQWs, that transition occurs between the two compressive strained layers. The advantages of the new strain compensated structure will be discussed below. 


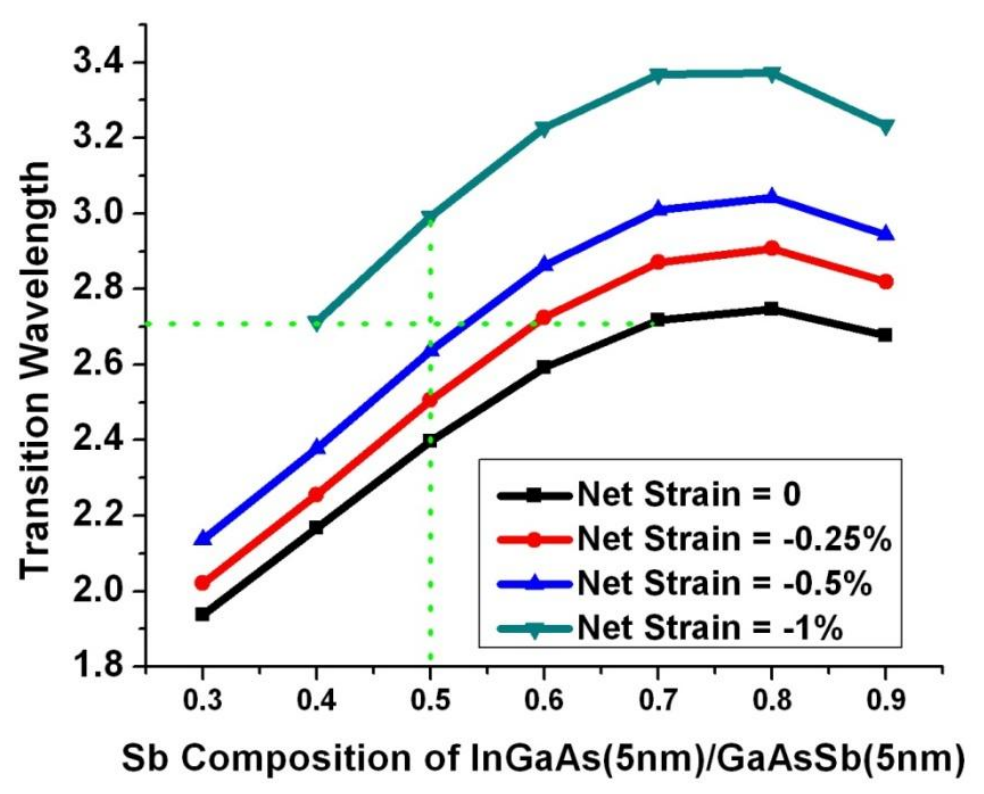

Figure 5.30 Transition wavelength v.s Sb composition of $5 \mathrm{~nm}$ InGaAs/5nm GaAsSb type-II quantum well with difference net compressive strain

Figure 5-30 plots the transition wavelength as function of $\mathrm{Sb}$ composition in the $5 \mathrm{~nm}$ InGaAs/5nm GaAsSb type-II quantum wells with different net strain, which is simply defined as below:

$$
\varepsilon=\frac{\sum_{i=1}^{n} \varepsilon_{i} t_{i}}{\sum_{i=1}^{n} t_{i}}
$$

As indicated in Figure 5.30, with net compressive strain in the layers where the type-II transition takes place, the detection wavelength is expected to be longer than that of zero net strain structures (conventional strain compensated structure) with the same $\mathrm{Sb}$ composition in GaAsSb layer. Moreover, due to the well-known Sb segregation effect in the epitaxial growth process $[111,112]$, growth of GaAsSb with high Sb composition is challenging $[113,114]$. This approach shows the advantage over the previous strain 
compensated method by requiring lower $\mathrm{Sb}$ composition in GaAsSb layer while achieving similar detection wavelength.

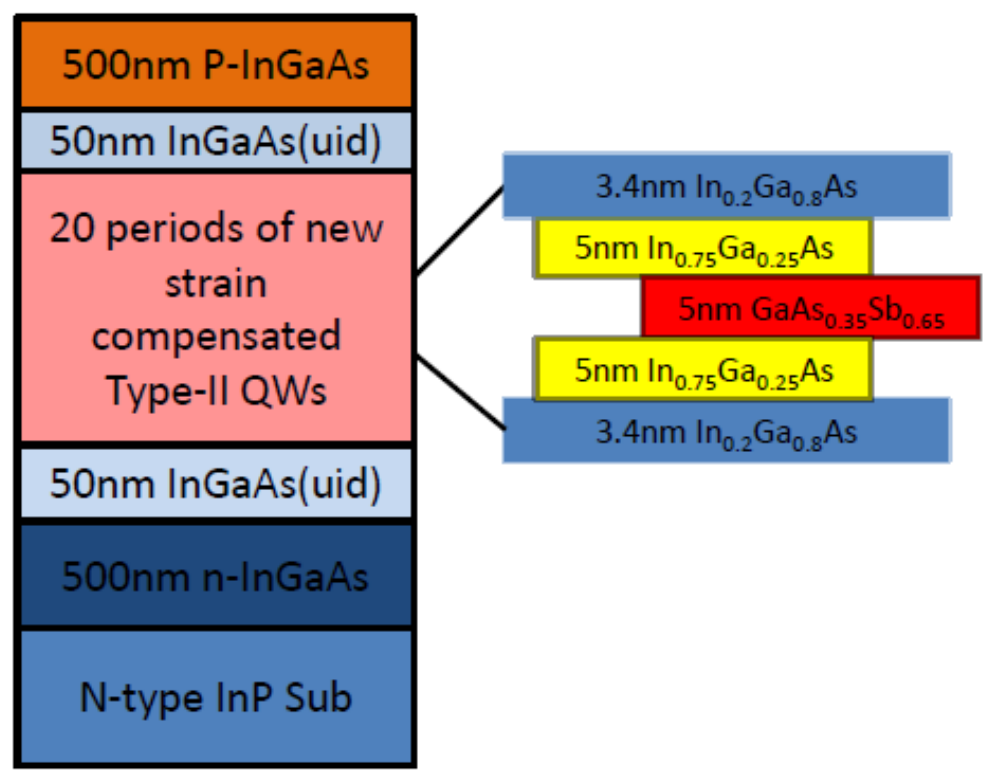

Figure 5.31 Schematic diagram for Sample C

A new strain compensated structure (sample C) is designed as shown in Figure 5-31. I used two $5 \mathrm{~nm} \operatorname{In}_{0.75} \mathrm{Ga}_{0.25} \mathrm{As}$ layer and one $5 \mathrm{~nm} \mathrm{GaAs}_{0.35} \mathrm{Sb}_{0.65}$ as the compressively strained quantum well for MWIR absorption, and used $6.8 \mathrm{~nm}$ tensile strained $\operatorname{In}_{0.2} \mathrm{Ga}_{0.8} \mathrm{As}$ barrier to compensate the compressively strain quantum well. Given the complication of this structure, only 20 periods of quantum wells are initially designed and grown. Material composition and thickness of layers were verified using high resolution X-ray diffraction (HRXRD) as shown in Figure 5-32. Compared the X-ray diffraction experimental results with fitting data, it was found that both the In composition in the compressive InGaAs layer and $\mathrm{Sb}$ composition in the compressive GaAsSb layer are 
lower than the design values. According to the new fitted composition values, the transitions between the lowest energy states in InGaAs and GaAsSb for Sample C are expected to occur around $0.41 \mathrm{eV}$, which corresponds to a cut-off wavelength of $3.0 \mu \mathrm{m}$.

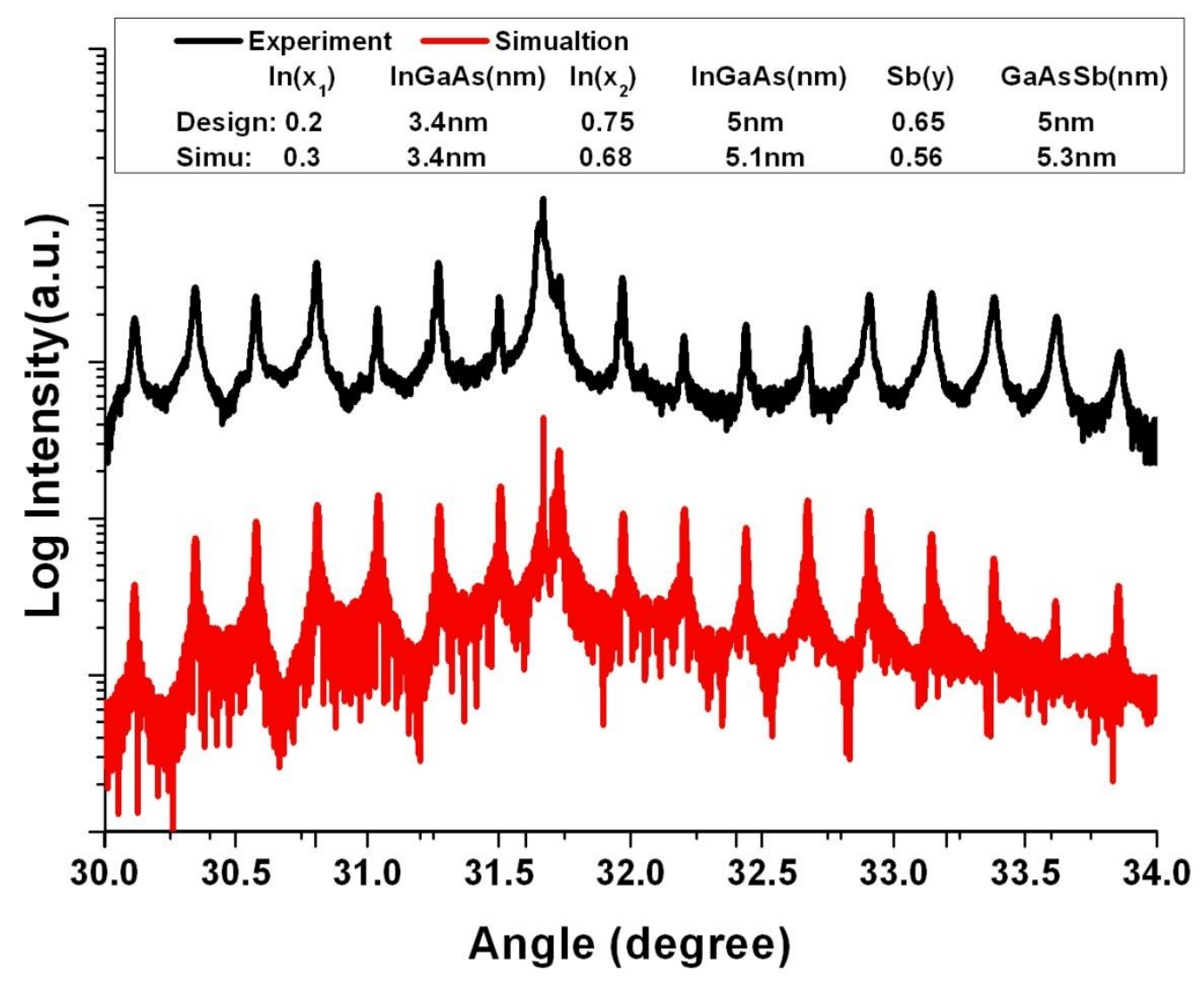

Figure 5.32 HRXRD data of Sample C of 20 pairs of type-II quantum wells

\subsubsection{Characteristics}

The dark current-voltage (I-V) characteristics of the device with $130 \mu \mathrm{m}$ diameter are shown in Figure 5-33. Device has the dark current density of $2.6 \mathrm{~mA} / \mathrm{cm}^{2}$ at $290 \mathrm{~K}$ at $0.5 \mathrm{~V}$, and decrease to $6.63 \mu \mathrm{A} / \mathrm{cm}^{2}$ at $200 \mathrm{~K}$. The dark current density of this device is 
slightly lower than that of the conventional strain compensated Sample B, due to the fact that this structure has a thinner absorption region. An Arrhenius plot of the dark current at $-0.5 \mathrm{~V}$ is shown in Figure 5-34. A good exponential fit to the data suggests that single activation energy of around $0.33 \mathrm{eV}$ dominates the dark current. Also the values of $\mathrm{R}_{0} \mathrm{~A}$ product for the device at different temperatures are shown in Figure 5-34. The $\mathrm{R}_{0} \mathrm{~A}$ product at $290 \mathrm{~K}$ is $42 \Omega \cdot \mathrm{cm}^{2}$, and it increases to $16800 \Omega \cdot \mathrm{cm}^{2}$ at $200 \mathrm{~K}$. From $200 \mathrm{~K}$ to $290 \mathrm{~K}$, the $\mathrm{R}_{0} \mathrm{~A}$ shows an Arrhenius type behavior with activation energy of $0.33 \mathrm{eV}$, which is very close to the activation energy of the dark current at $-0.5 \mathrm{~V}$.

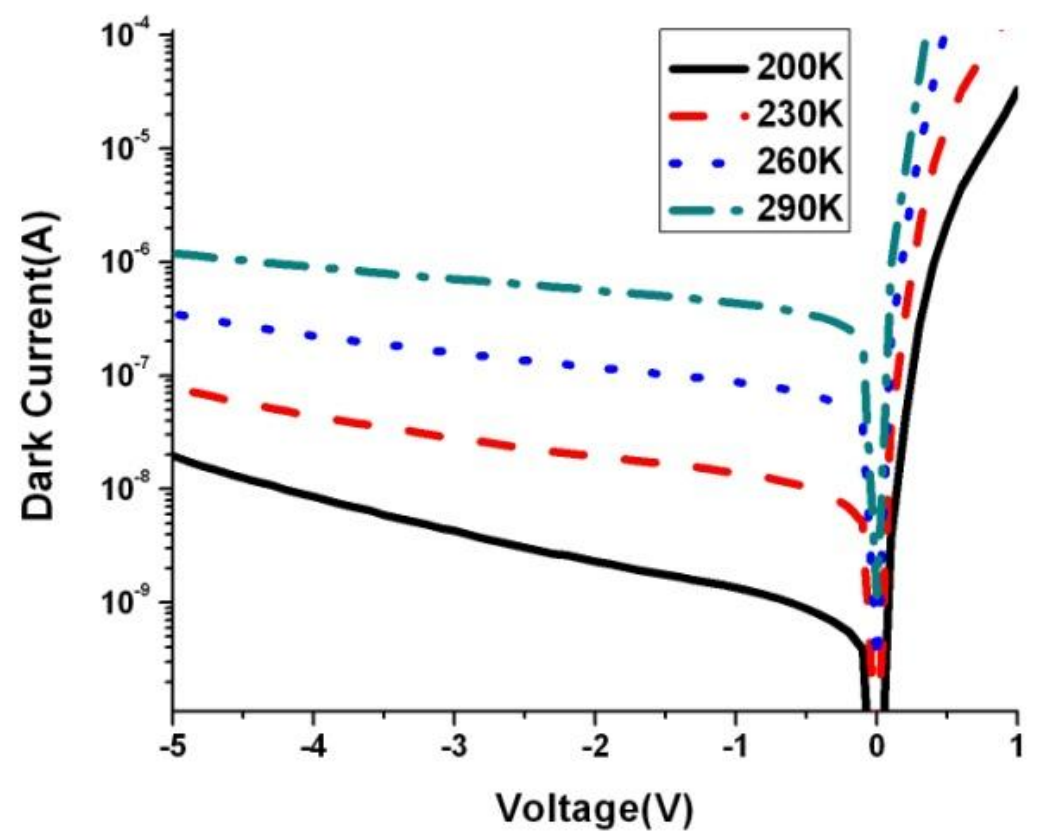

Figure 5.33 Dark current of Sample C versus voltage with $130 \mu \mathrm{m}$ diameter measured at different temperatures 


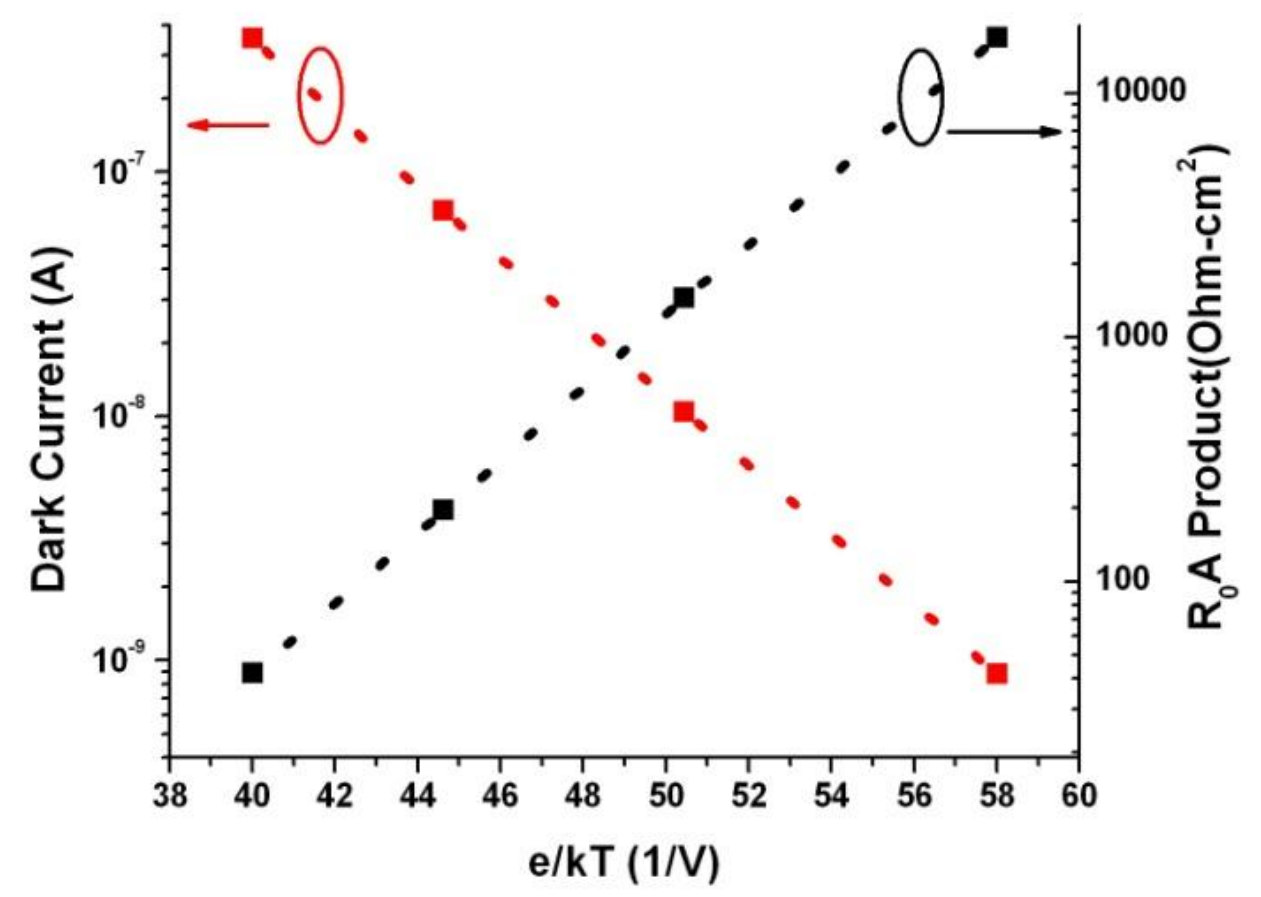

Figure 5.34 Arrhenius plots of the dark current at $-0.5 \mathrm{~V}$ and the $\mathrm{R}_{0} \mathrm{~A}$ product for $130 \mu \mathrm{m}$ diameter Sample C

\subsubsection{Optical Characteristics}

The normal incident photo response at $0 \mathrm{~V}$ and $-0.5 \mathrm{~V}$ biases are measured at temperatures between $200 \mathrm{~K}$ and $290 \mathrm{~K}$ as shown in Figure 5-35(a) and Figure 5-35(b). The device has optical response out to $3.1 \mu \mathrm{m}$ at $290 \mathrm{~K}$, close to the theoretical prediction based on the XRD fitted composition values. The response peak red shifts with an increase in temperature, because of the band gap reduction of each quantum well layer at higher temperature. It is consistent with the phenomena of Sample A and Sample B. As we can see in Figure 5-35, the responsivity increases when the device is reversed biased. Further increase in reverse bias beyond $-0.5 \mathrm{~V}$ does not significantly increase the device responsivity. 


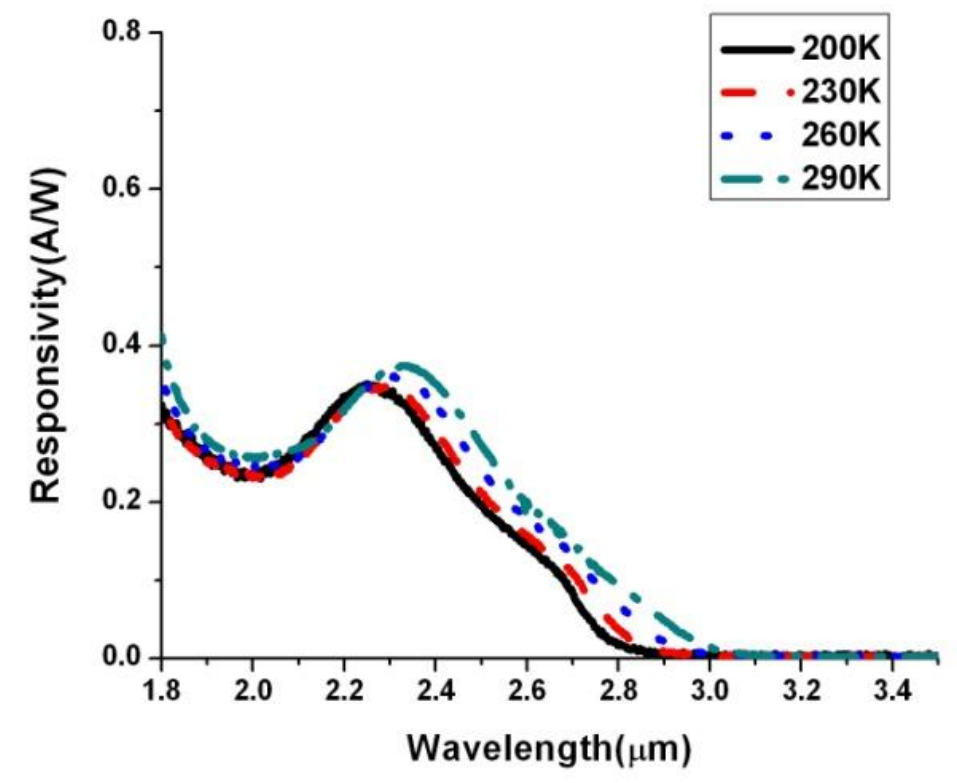

(a)

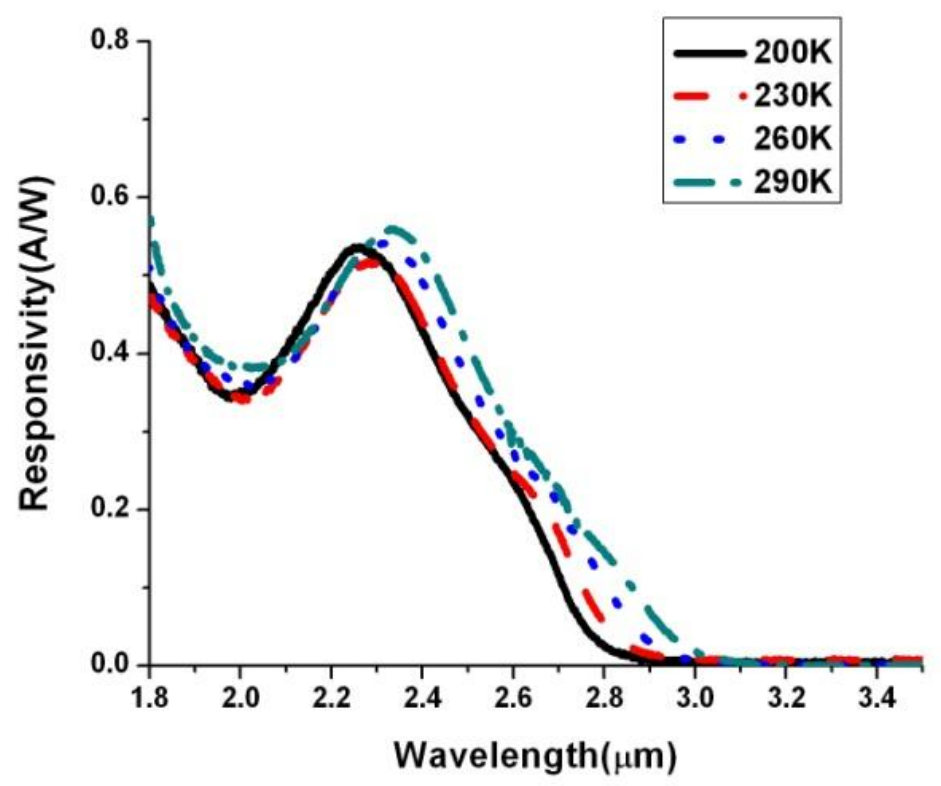

(b)

Figure 5.35 Normal incident photo response of the sample $\mathbf{C}$ at different temperatures (a) with zero reverse bias, (b) with $0.5 \mathrm{~V}$ reverse bias 


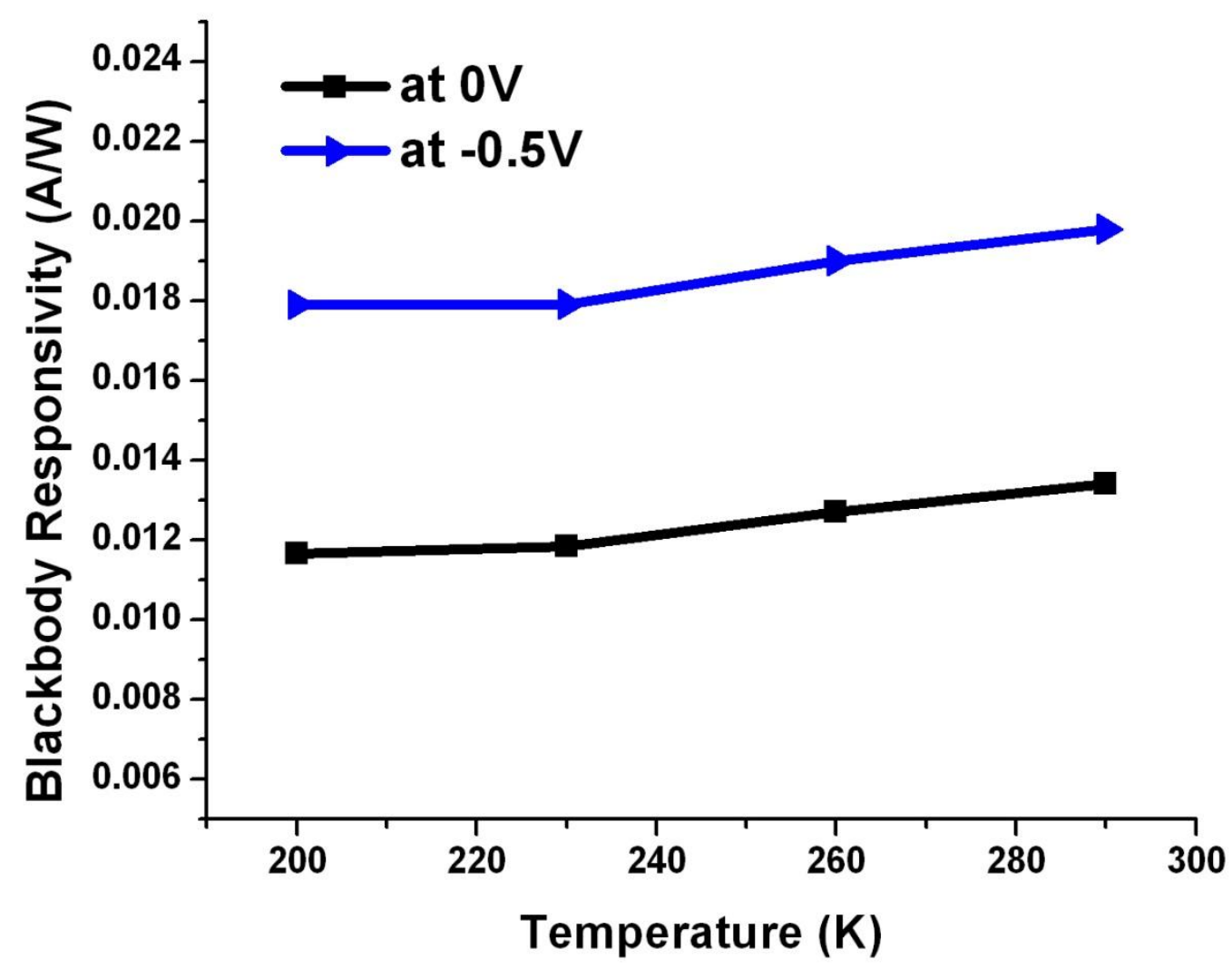

Figure 5.36 Blackbody responsivity of Sample C under different bias versus temperature

A unique feature of the Sample C is that its responsivity decreases as the temperature decreases which is a different than observed in Samples A and B. There is no critical temperature point as in Sample A and Sample B shown in Figure 5-9 and Figure 5-22. As the blackbody responsivity shown in Figure $5-36$, the responsivity at $-0.5 \mathrm{~V}$ is always higher than the responsivity at $0 \mathrm{~V}$ under temperature from $200 \mathrm{~K}$ to $290 \mathrm{~K}$, which indicates that higher electrical field is necessary to improve carrier collection efficiency. This behavior is also different from that of Sample A and Sample B. The unique band structure of Sample C due to additional tensile barrier could be the cause of these effects. As shown in Figure 5-37 for the band alignment of Sample C, the conduction band of 
tensile InGaAs barrier is lower than the conduction of GaAsSb layer. The photo generated carriers could be recaptured in the tensile InGaAs barrier and get recombined with defects after getting out of the compressive InGaAs quantum well if the electrical field is low. Under high electrical field, the photo generated carriers could be swept across the absorption region faster and have lower possibility to get recaptured. This is why responsivity under low bias is always lower than the responsivity under high bias for temperature from 200K to 290K. Therefore, it can be concluded that Sample C requires a higher electrical field than that of Sample A and Sample B, in order to collect the photo generated carriers efficiently. This may be undesirable, since higher electrical fields can lead to an increase in the dark current and dark noise of the device.

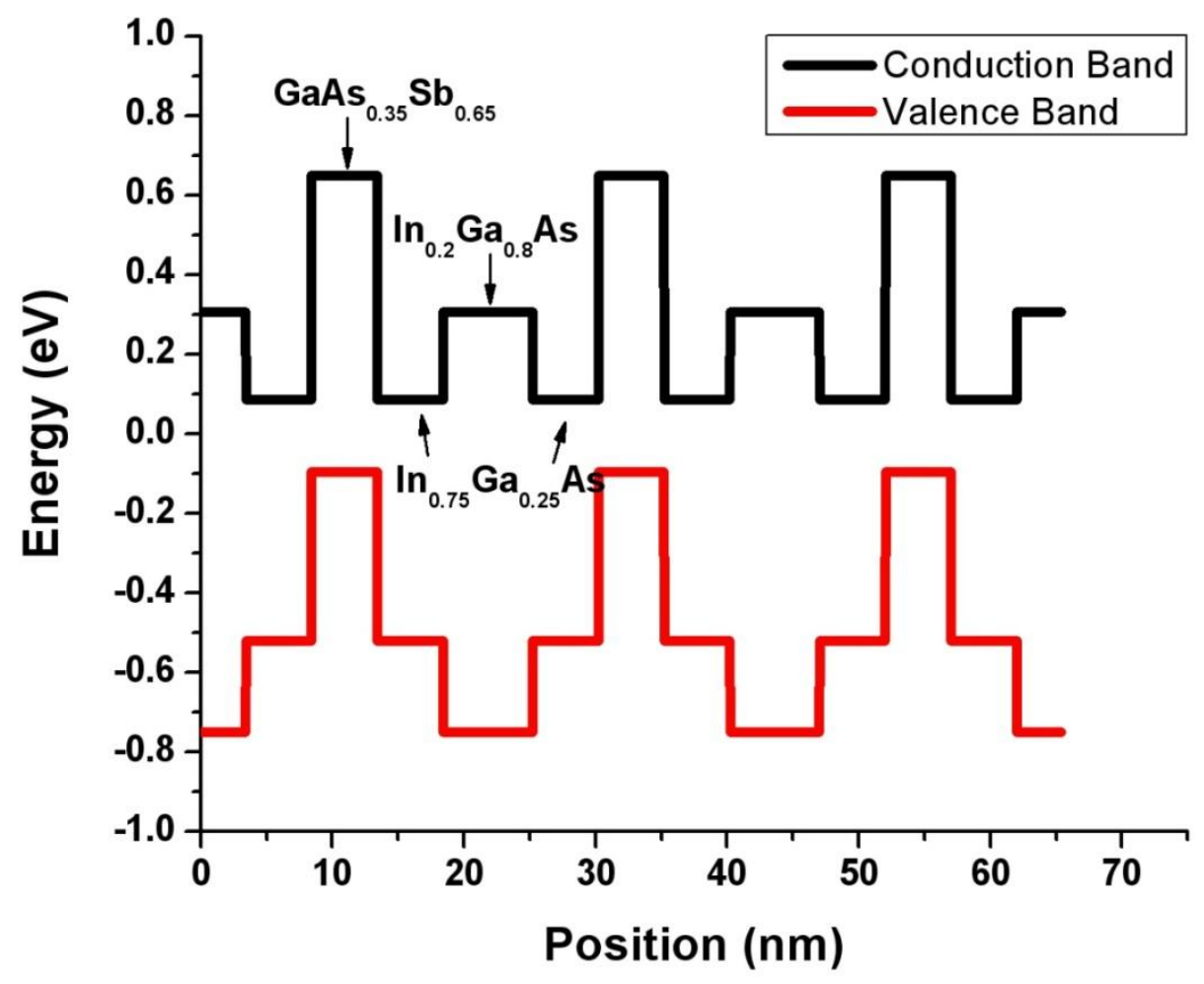

Figure 5.37 Band Alignment of the new strain compensated quantum wells in Sample C 
The dark noise spectrum densities of Sample C versus temperature under both 0V bias and $-0.5 \mathrm{~V}$ bias were also measured as shown in Figure 5-38. Compared with dark noise performance of Sample A and Sample B, the noise of Sample C does not decrease significantly when temperature decreases. The potential reason may be due to the high defect density in the absorption region based on the certification data from IQE. Further optimizing the growth condition would be necessary in order to get the good quality epilayer.

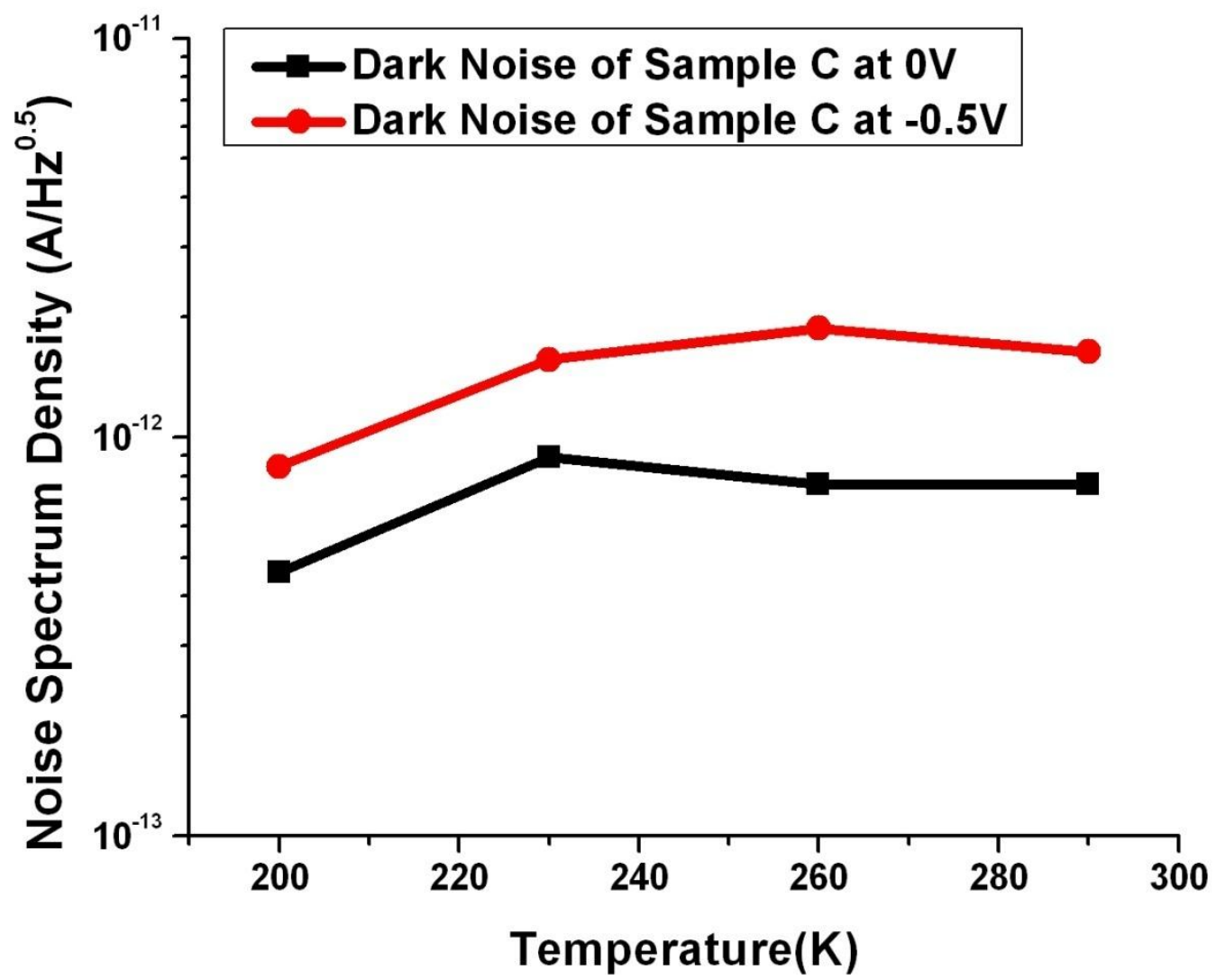

Figure 5.38 Dark noise spectrum densities versus temperature under $0 \mathrm{~V}$ bias and $\mathbf{- 0 . 5 \mathrm { V }}$ bias for Sample C 
The specific detectivity $\left(D^{*}\right)$ versus wavelength at $290 \mathrm{~K}$ is shown in Figure 5-39 compared with that of Sample B. D* for Sample C is $2.3 \times 10^{9} \mathrm{~cm} \cdot \mathrm{Hz}^{1 / 2} \cdot \mathrm{W}^{-1}$ at $\lambda=2.7 \mu \mathrm{m}$ at 290K under 0V bias, but this value does not increase when temperature decreases to 200K, due to the noise performance. This value is comparable with the detectivity of Sample B at $\lambda=2.7 \mu \mathrm{m}$ at $290 \mathrm{~K}$ under optimized bias $(-0.5 \mathrm{~V})$, which is $1.4 \times 10^{9}$ $\mathrm{cm} \cdot \mathrm{Hz}^{1 / 2} \cdot \mathrm{W}^{-1}$.

In conclusion, the new strain compensation approach used in Sample C can be used in terms of extending detection wavelength or reducing the $\mathrm{Sb}$ composition in GaAsSb layers. However, epilayer growth condition of Sample C still needs further improvement in order to achieve desirable performance.

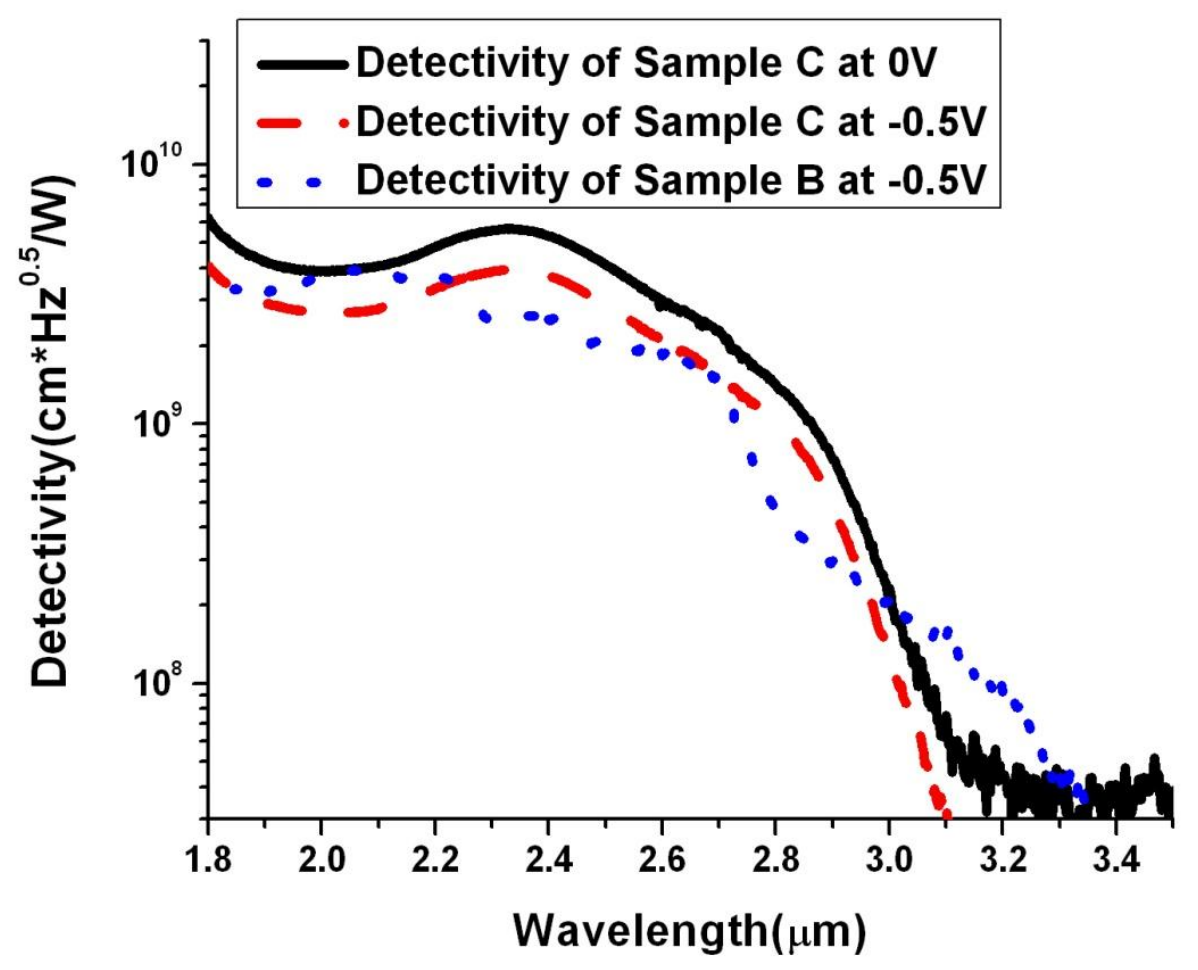

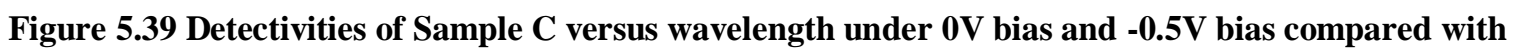
that of Sample B at 290K 


\subsection{Summary}

I have discussed three InP based InGaAs/GaAsSb type-II quantum wells photodiodes in this chapter. Systematic studies on electrical and optical characteristics of these devices were performed. The important parameters of these photodiodes are summarized in Table 5-3 as below.

Table 5-3 Performance of different InP based type-II Quantum wells Photodiodes

\begin{tabular}{|c|c|c|c|c|}
\hline Device & Dark Current Density & $\mathrm{R}_{0} \mathrm{~A}\left(\mathrm{Ohm} \cdot \mathrm{cm}^{2}\right)$ & $\begin{array}{l}\text { Cut-off } \\
\text { wavelength }\end{array}$ & $\begin{array}{l}\text { Specific } \\
\text { Detectivity }\end{array}$ \\
\hline \multirow[t]{2}{*}{$\begin{array}{l}\text { Sidhu's } \\
\text { device [34] }\end{array}$} & $5 \mathrm{~mA} / \mathrm{cm}^{2}$ at $295 \mathrm{~K}$ & $50 \mathrm{Ohm} \cdot \mathrm{cm}^{2}$ at $295 \mathrm{~K}$ & \multirow[t]{2}{*}{$\begin{array}{l}\text { Around } 2.5 \mu \mathrm{m} \\
\text { at } 295 \mathrm{~K}\end{array}$} & $\begin{array}{l}3.8 \times 10^{9} \text { Jones at } \\
2.2 \mu \mathrm{m} \text { at } 295 \mathrm{~K}\end{array}$ \\
\hline & $13.3 \mu \mathrm{A} / \mathrm{cm}^{2}$ at $200 \mathrm{~K}$ & NA & & $\begin{array}{l}5.6 \times 10^{10} \text { Jones at } \\
2.17 \mu \mathrm{m} \text { at } 200 \mathrm{~K}\end{array}$ \\
\hline \multirow[t]{2}{*}{ Sample A } & $1.66 \mathrm{~mA} / \mathrm{cm}^{2}$ at $290 \mathrm{~K}$ & $50 \mathrm{Ohm} \cdot \mathrm{cm}^{2}$ at $290 \mathrm{~K}$ & \multirow[t]{2}{*}{$\begin{array}{l}\text { Around } 2.7 \mu \mathrm{m} \\
\text { at } 290 \mathrm{~K}\end{array}$} & $\begin{array}{l}3.28 \times 10^{9} \text { Jones at } \\
2.4 \mu \mathrm{m} \text { at } 290 \mathrm{~K}\end{array}$ \\
\hline & $4.89 \mu \mathrm{A} / \mathrm{cm}^{2}$ at $200 \mathrm{~K}$ & $18,000 \mathrm{Ohm} \cdot \mathrm{cm}^{2}$ at $200 \mathrm{~K}$ & & $\begin{array}{l}1.51 \times 10^{11} \text { Jones at } \\
2.4 \mu \mathrm{m} \text { at } 200 \mathrm{~K}\end{array}$ \\
\hline \multirow[t]{2}{*}{ Sample B } & $9.7 \mathrm{~mA} / \mathrm{cm}^{2}$ at $290 \mathrm{~K}$ & $72 \mathrm{Ohm} \cdot \mathrm{cm}^{2}$ at $290 \mathrm{~K}$ & \multirow[t]{2}{*}{$\begin{array}{l}\text { Around } 3.2 \mu \mathrm{m} \\
\text { at } 290 \mathrm{~K}\end{array}$} & $\begin{array}{l}1.4 \times 10^{9} \text { Jones at } \\
2.7 \mu \mathrm{m} ; \\
2.0 \times 10^{8} \text { Jones at } \\
3 \mu \mathrm{m} \text { at } 290 \mathrm{~K}\end{array}$ \\
\hline & $58.6 \mu \mathrm{A} / \mathrm{cm}^{2}$ at $200 \mathrm{~K}$ & $2,090 \mathrm{Ohm} \cdot \mathrm{cm}^{2}$ at $200 \mathrm{~K}$ & & $\begin{array}{l}1.5 \times 10^{10} \text { Jones at } \\
2.7 \mu \mathrm{m} ; \\
1.0 \times 10^{9} \text { Jones at } \\
3 \mu \mathrm{m} \text { at } 200 \mathrm{~K}\end{array}$ \\
\hline \multirow[t]{2}{*}{ Sample C } & $2.6 \mathrm{~mA} / \mathrm{cm}^{2}$ at $290 \mathrm{~K}$ & $42 \Omega \cdot \mathrm{cm}^{2} 290 \mathrm{~K}$ & \multirow{2}{*}{$\begin{array}{l}\text { Around } 3.1 \mu \mathrm{m} \\
\text { at } 290 \mathrm{~K}\end{array}$} & $2.3 \times 10^{9}$ Jones at \\
\hline & $6.63 \mu \mathrm{A} / \mathrm{cm}^{2}$ at $200 \mathrm{~K}$ & $16800 \Omega \cdot \mathrm{cm}^{2}$ at $200 \mathrm{~K}$ & & $\lambda=2.7 \mu \mathrm{m}$ at $290 \mathrm{~K}$ \\
\hline
\end{tabular}


The lattice matched Sample A has optical response out to $2.7 \mu \mathrm{m}$, which is longer than that of the device with lattice matched $5 \mathrm{~nm}$ InGaAs/ $5 \mathrm{~nm}$ GaAsSb quantum wells absorption. The detectivity for Sample A is $3.28 \times 10^{9} \mathrm{~cm} \cdot \mathrm{Hz}^{1 / 2} \cdot \mathrm{W}^{-1}$ at $\lambda=2.4 \mu \mathrm{m}$ at $290 \mathrm{~K}$ and increases to $1.51 \times 10^{11} \mathrm{~cm} \cdot \mathrm{Hz}^{1 / 2} \cdot \mathrm{W}^{-1}$ at $200 \mathrm{~K}$. The strain compensated Sample B has an optical response out to wavelength of $3.2 \mu \mathrm{m}$, which is longer than that of Sample A, as we expected. The detectivity for Sample B is $2.0 \times 10^{8} \mathrm{~cm} \cdot \mathrm{Hz}^{1 / 2} \cdot \mathrm{W}^{-1}$ at $\lambda=3.0 \mu \mathrm{m}$ at $290 \mathrm{~K}$ and increases to $1.0 \times 10^{9} \mathrm{~cm} \cdot \mathrm{Hz}^{1 / 2} \cdot \mathrm{W}^{-1}$ at $200 \mathrm{~K}$. The new strain compensated Sample C is initially designed in order to extend the detection wavelength or reducing the $\mathrm{Sb}$ composition in GaAsSb layers. And the detectivity performance of Sample C at 290K is comparable with that of Sample B. However, given the crystal quality, no improvement of the performance of Sample C was observed at lower temperature. Therefore, further improvement of the crystal quality is necessary in order to explore the full advantage of this new strain compensated design. 


\section{Chapter 6: Carrier Dynamics Study of InP based PIN Photodiodes with InGaAs/GaAsSb Type-II}

\section{Quantum Wells}

\subsection{Introduction}

One of the limitations of these InP based type-II quantum wells photodiodes is their quantum efficiency. For a PIN photodiodes, quantum efficiency $\left(\eta_{\text {ext }}\right)$ is given as in Chapter 4.

$$
\eta=(1-R)\left(1-e^{-\alpha d}\right) \eta_{\text {coll }}
$$

where $\eta_{\text {coll }}$ is carrier collection efficiency, $R$ is the optical reflectivity, $\alpha$ is the absorption coefficient of the light and $\mathrm{d}$ is the width of the absorption region. The optical reflectivity can be minimized by the using an antireflection coating [115], and light absorption can be enhanced by increasing the thickness of the absorption region. In order to further improve the quantum efficiency, the carrier transport mechanism needs to be well understood in order to improve the carrier collection efficiency.

In this chapter, two structures were designed to study the effects of thermionic emission and phonon assisted tunneling on the performance of InP based photodiodes with InGaAs/GaAsSb type-II quantum well absorption regions. Based on the comparison of the responsivity of the two different device structures, I show that thermionic emission is the dominant carrier transport mechanism for these quantum well structures. 


\subsection{Carrier Collection Efficiency Modeling}

To understand effects that these two thermionic emission and phonon assisted tunneling have on the quantum efficiency, I created a model to calculate the carrier collection efficiency any photodiode with quantum well absorption regions.

In order to accurately model the carrier dynamics of an $\mathrm{N}$ period quantum wells absorption region, I defined a set of rate equations describing carriers which, once they escape from a well, can either be captured by the left or right adjacent well (with probabilities of 1- $\mathrm{p}_{\mathrm{l}}$ and $1-\mathrm{p}_{\mathrm{r}}$ respectively ) as below.

$$
\begin{aligned}
\frac{\mathrm{dq}_{\mathrm{k}}}{\mathrm{dt}}=\mathrm{G}_{\mathrm{k}}-\left(\frac{1}{\tau_{1}}\right. & \left.+\frac{1}{\tau_{\mathrm{r}}}+\frac{1}{\tau}+\frac{1}{\tau_{\mathrm{t}}}\right) \mathrm{q}_{\mathrm{k}} \\
& +\frac{1-\mathrm{p}_{\mathrm{l}}}{\mathrm{p}_{\mathrm{l}}} \sum_{\mathrm{m} \geq 1} \frac{\mathrm{p}_{\mathrm{l}}^{\mathrm{m}}}{\left(\tau_{1}+\mathrm{m} \times \mathrm{t}_{\text {drift }}\right)} \mathrm{q}_{\mathrm{k}+\mathrm{m}}+\frac{1}{\tau_{\mathrm{t}}} \mathrm{q}_{\mathrm{k}+1} \\
& +\frac{1-\mathrm{p}_{\mathrm{r}}}{\mathrm{p}_{\mathrm{r}}} \sum_{\mathrm{m} \geq 1} \frac{\mathrm{p}_{\mathrm{r}}^{\mathrm{m}}}{\left(\tau_{\mathrm{r}}+\mathrm{m} \times \mathrm{t}_{\text {drift }}\right)} \mathrm{q}_{\mathrm{k}+\mathrm{m}}
\end{aligned}
$$

In equation (6.2), $\mathrm{q}_{\mathrm{k}}(\mathrm{t})$ represents the number of carriers in the $\mathrm{k}^{\text {th }}$ well $(\mathrm{k}=1,2,3, \ldots$, $\mathrm{N}), \tau$ is the carrier lifetime, $\tau_{1}$ and $\tau_{\mathrm{r}}$ are the thermionic emission time to overcome the left and right barrier respectively, $\tau_{\mathrm{t}}$ is the phonon assisted tunneling time from the $\mathrm{k}+1^{\text {th }}$ well to the kth well, which is the mean tunneling time of the electrons, $G_{k}=I_{k} \cdot \alpha \cdot d$ is the generation rate in the $\mathrm{k}^{\text {th }}$ well due to the absorption of light, $\mathrm{t}_{\text {drift }}$ is the time to drift one period of quantum well. $\mathrm{I}_{\mathrm{k}}$ is optical photo flux rate in the $\mathrm{k}^{\text {th }}$ well, and $\alpha$ is the absorption coefficient.

These equations can be simultaneously solved for $\mathrm{q}_{\mathrm{k}}$ under steady state conditions and used to calculate the quantum efficiency $(\eta)$ and carrier collection efficiency $\left(\eta_{\text {coll }}\right)$ with the following expressions: 


$$
\begin{gathered}
\eta=\sum_{i=1}^{N} q_{i} \cdot \frac{p_{l}^{i-1}}{\tau_{l}+(i-1) t_{d r i f t}} \\
\eta_{\text {coll }}=\frac{\eta}{1-e^{-\alpha d}}
\end{gathered}
$$

The thermionic emission time $\tau_{1}, \tau_{\mathrm{r}}$ was derived in [116] as shown below, assuming a thermal distribution of carriers.

$$
\tau_{\mathrm{l}, \mathrm{r}}=\left(\frac{2 \pi \mathrm{m}_{\mathrm{e}} \mathrm{L}_{\mathrm{QW}}^{2}}{\mathrm{k}_{\mathrm{B}} \mathrm{T}}\right)^{1 / 2} \exp \left[\frac{\mathrm{E}_{\mathrm{l}, \mathrm{r}}^{\mathrm{b}}-\mathrm{E}_{1}}{\mathrm{k}_{\mathrm{B}} \mathrm{T}}\right]
$$

In this expression, $m_{e}$ is the effective mass of electrons in the quantum well, $E_{1}$ is the lowest lying quasi-bound state in the well, $\mathrm{E}_{\mathrm{l}, \mathrm{r}}^{\mathrm{b}}$ is the left or right barrier energy. This expression is derived using the assumption that only the ground well state is populated. This assumption is applicable for the photodiodes, since the quantum efficiency of photodiodes is generally measured with small optical power illumination. The phonon assisted tunneling time is hard to calculate from first principles since it depends on a number of factors that are not well known (e.g., the in-plane wave vector $\mathrm{k}_{\mathrm{i}}$ of the initial and the final subband state [102]). As a result, I used $\tau_{\mathrm{t}}$ to represent the average phonon assisted tunneling time.

These equations (6.1-6.5) can be used to study the dynamic of thermionic emission and phonon assisted tunneling process in the multiple quantum wells (MQW) structure, if the thermionic emission time and phonon assisted tunneling time are known. Moreover, the quantum efficiency of MQW photodiodes can also be calculated. 


\subsection{Experiment Design}

Two different PIN photodiodes were designed, grown in an MBE reactor, fabricated, and characterized in order to study the carrier dynamics. Both samples use a one hundred pairs $\operatorname{In}_{0.53} \mathrm{Ga}_{0.47} \mathrm{As} / \mathrm{GaAs}_{0.5} \mathrm{Sb}_{0.5}$ quantum well absorption region. The thicknesses of the InGaAs and GaAsSb quantum well layers are $7 \mathrm{~nm}$ and $5 \mathrm{~nm}$ respectively. The difference between the two structures is the $50 \mathrm{~nm}$ layer adjacent to the $\mathrm{n}$-InGaAs contact layer. It is a $50 \mathrm{~nm} \mathrm{InP}$ layer for structure $\mathrm{D}$, and a $50 \mathrm{~nm}$ GaAsSb layer for structure $\mathrm{E}$ as shown below in Figure 6.1(a) and Figure 6.1(b).

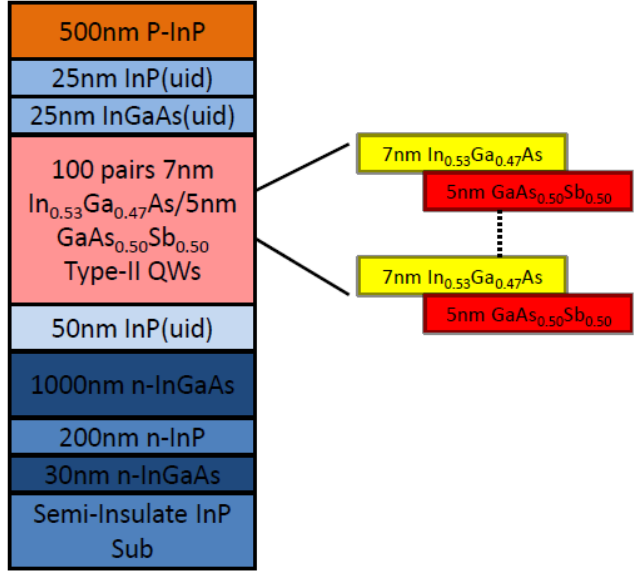

(a)

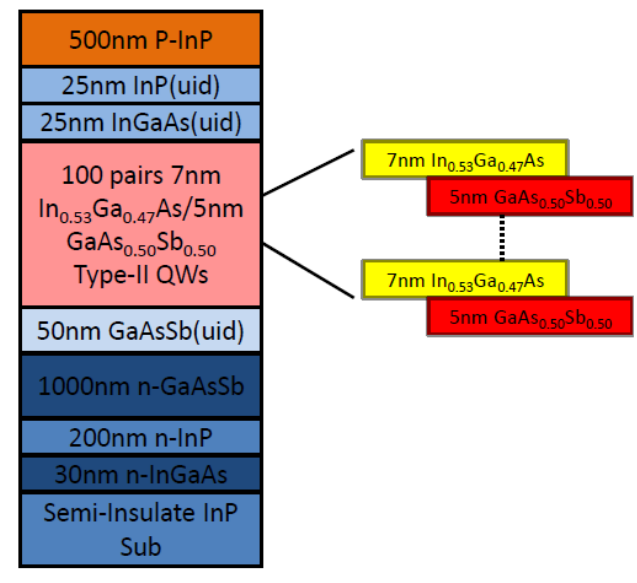

(b)

Figure 6.1 InP based type-II Quantum well photodiodes structure for carrier study (a) Sample D with a 50nm InP barrier (b) Sample E with a 50nm GaAsSb barrier

Since InP and GaAsSb have different energy heights with respect to the adjacent quantum wells, the effects of the two carrier transport mechanism can be studied. If phonon-assisted tunneling is the dominant carrier mechanism, the expected carrier transport for absorbed carriers is shown in Figure 6.2. 


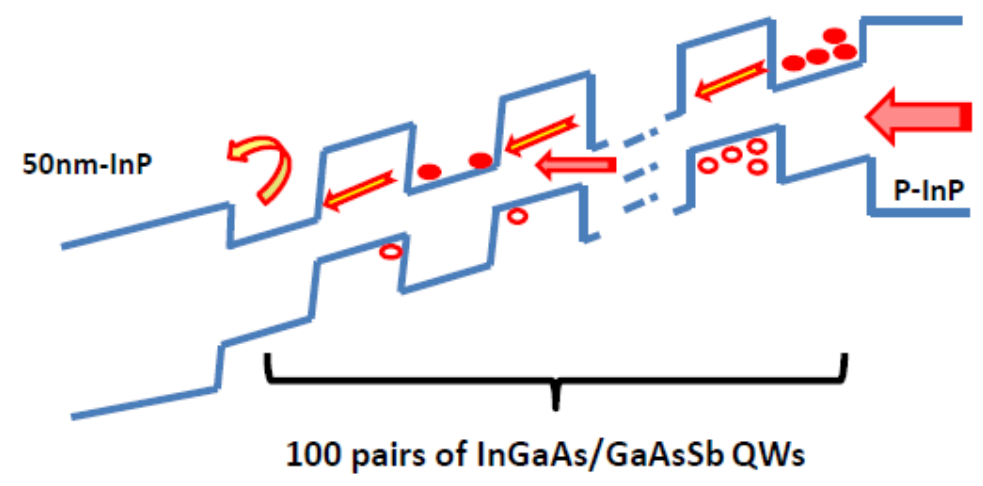

(a)

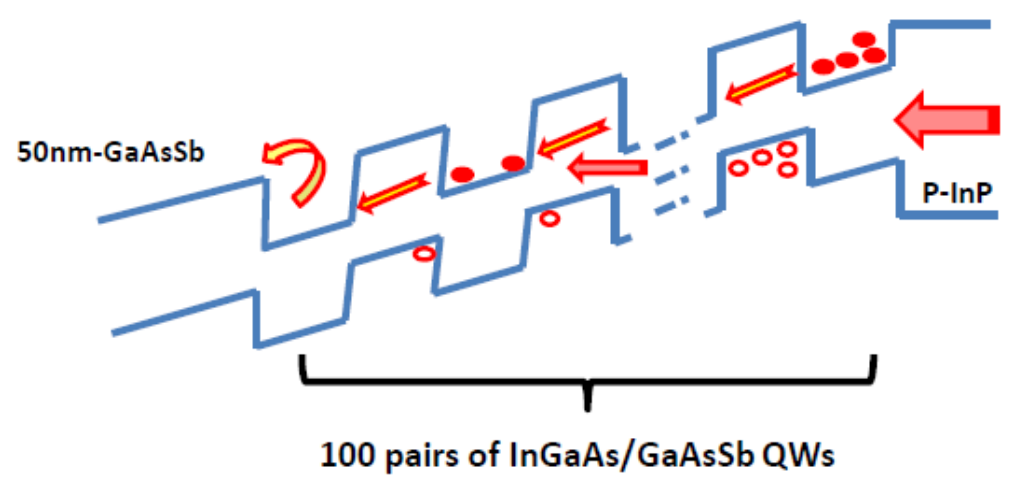

(b)

Figure 6.2 (a) Carrier transport in type-II quantum well structure in sample $D$ if phonon-assisted tunneling dominates (b) Carrier transport in type-II quantum well structure in sample $\mathbf{E}$ if phononassisted tunneling dominates

In Figure 6.2, we can see electrons transport to the last quantum well before the nregion by phonon assisted tunneling. At this point, the only way for these electrons to be collected is by thermionic emission, since the last barrier (50nm InP or GaAsSb) is too thick for efficient tunneling. Given that GaAsSb has a higher barrier height; it is expected to have a smaller quantum efficiency, since carriers in sample E would stay in the last well for a longer time leading to a lower carrier collection efficiency. 
If thermionic emission is the dominant mechanism, the expected carrier transport for absorbed carriers is shown in Figure 6.3. Once the electrons leave a well, they travel with the energy above the barriers until they are collected. In this case, it is expected that no difference in quantum efficiency will be observed between the two structures.

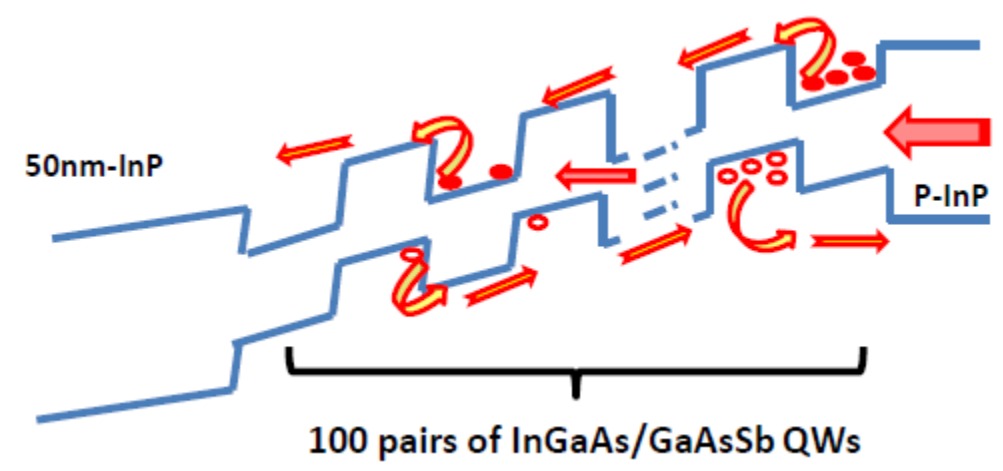

(a)

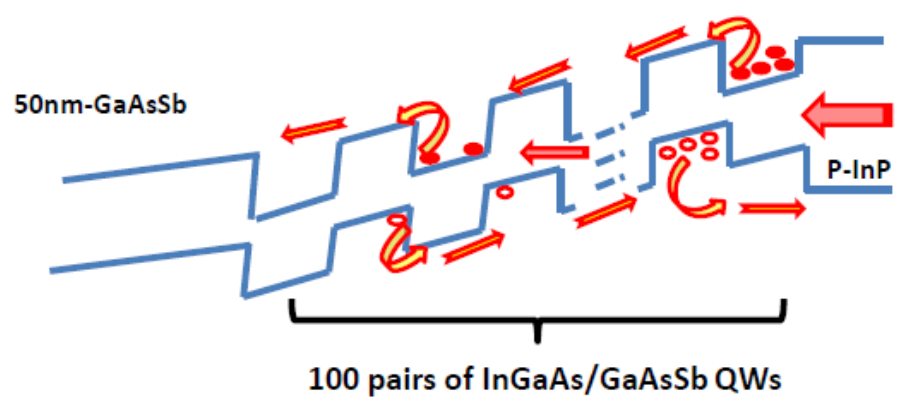

(b)

Figure 6.3 (a) Carrier transport in type-II quantum well structure in sample $D$ if thermionic emission dominates (b) Carrier transport in type-II quantum well structure in sample $E$ if thermionic emission dominates

The model developed in Section 6.2 was used to verify the analysis above. Here the following assumptions were made: the carrier lifetime is $10 \mathrm{~ns}$, capture probability by the left neighboring well is $0 \%$, capture probability by the right neighboring well is $100 \%$ 
[117, 118], the electrical field in the absorption region is $1.8 \times 10^{4} \mathrm{~V} / \mathrm{cm}$ and the absorption coefficient is $3000 \mathrm{~cm}^{-1}$. These values are typical values for PIN type-II quantum wells photodiodes based on the theoretical calculation [78] and experimental data $[34,80]$. Since the goal here is to investigate whether the phonon-assist tunneling or thermionic emission is the dominant carrier transport mechanism, the carrier collection efficiency as a function of the ratio between phonon-assist tunneling time and the thermionic emission time was calculated and shown in Figure 6.4 for Samples D and E.

From Figure 6.4, we can conclude that when phonon-assist tunneling is the dominant transport mechanism $(\mathrm{R}<<1)$, the carrier collection efficiency of Sample D will be larger than that of Sample E. If thermionic emission is the dominant transport mechanism $(\mathrm{R}>1)$, the carrier collection efficiencies of Samples D and E will be similar.

Generally speaking, the method used here to study the carrier dynamics in MQWs, is not just limited to these InP based type-II MQWs, and it can be generalized to any other MQWs as long as the lattice matched materials with different energy barriers are available on such substrate. 


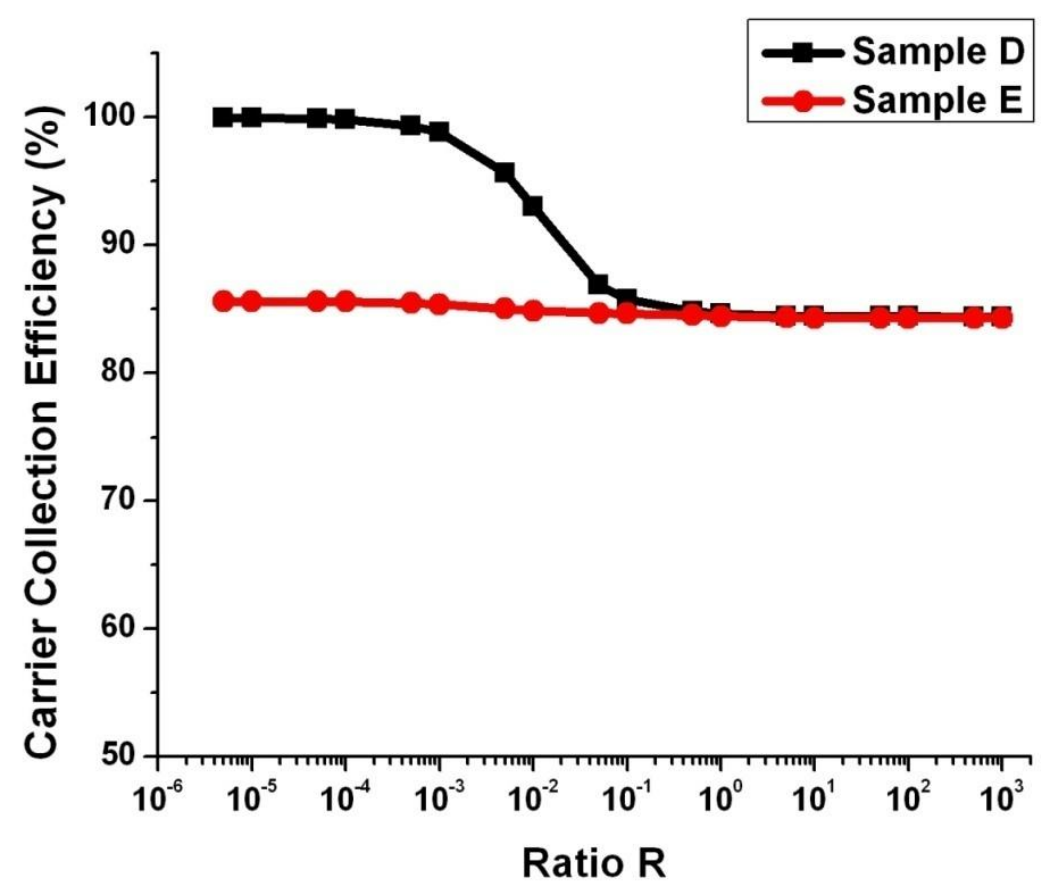

Figure 6.4 the carrier collection efficiency as a function of the ratio between phonon-assist tunneling time and the thermionic emission time

\subsection{Experimental Results}

Material composition and thickness of layers for Samples D and E were verified using high resolution X-ray diffraction (HRXRD) as shown in Figure 6.5. The appearances of a large number of satellite peaks suggest that both structures are of high quality and have abrupt interfaces in the absorption region. Moreover, the positions of satellite peaks in both samples are similar, which indicates the type-II quantum wells in absorption region of both samples should have similar material compositions, layer thicknesses, and absorption beyond 1800nm. 


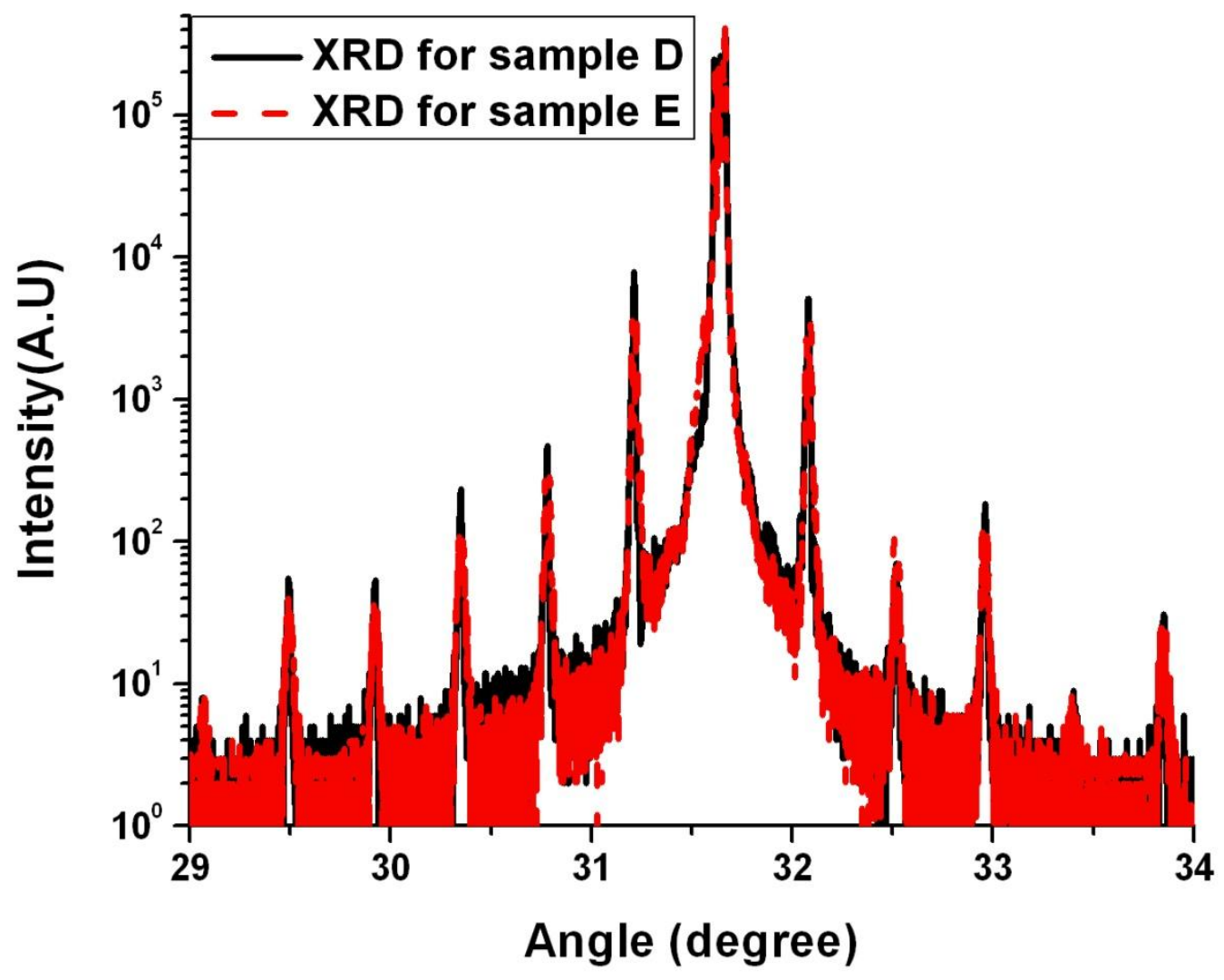

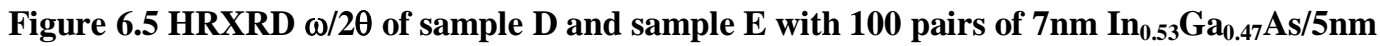
$\mathbf{G a A s}_{0.5} \mathbf{S b}_{0.5}$

Devices with top-illuminated mesas were fabricated (see Appendix A for details). No antireflection coating was used for either device, in order to minimize the uncertainty in the measurements. 


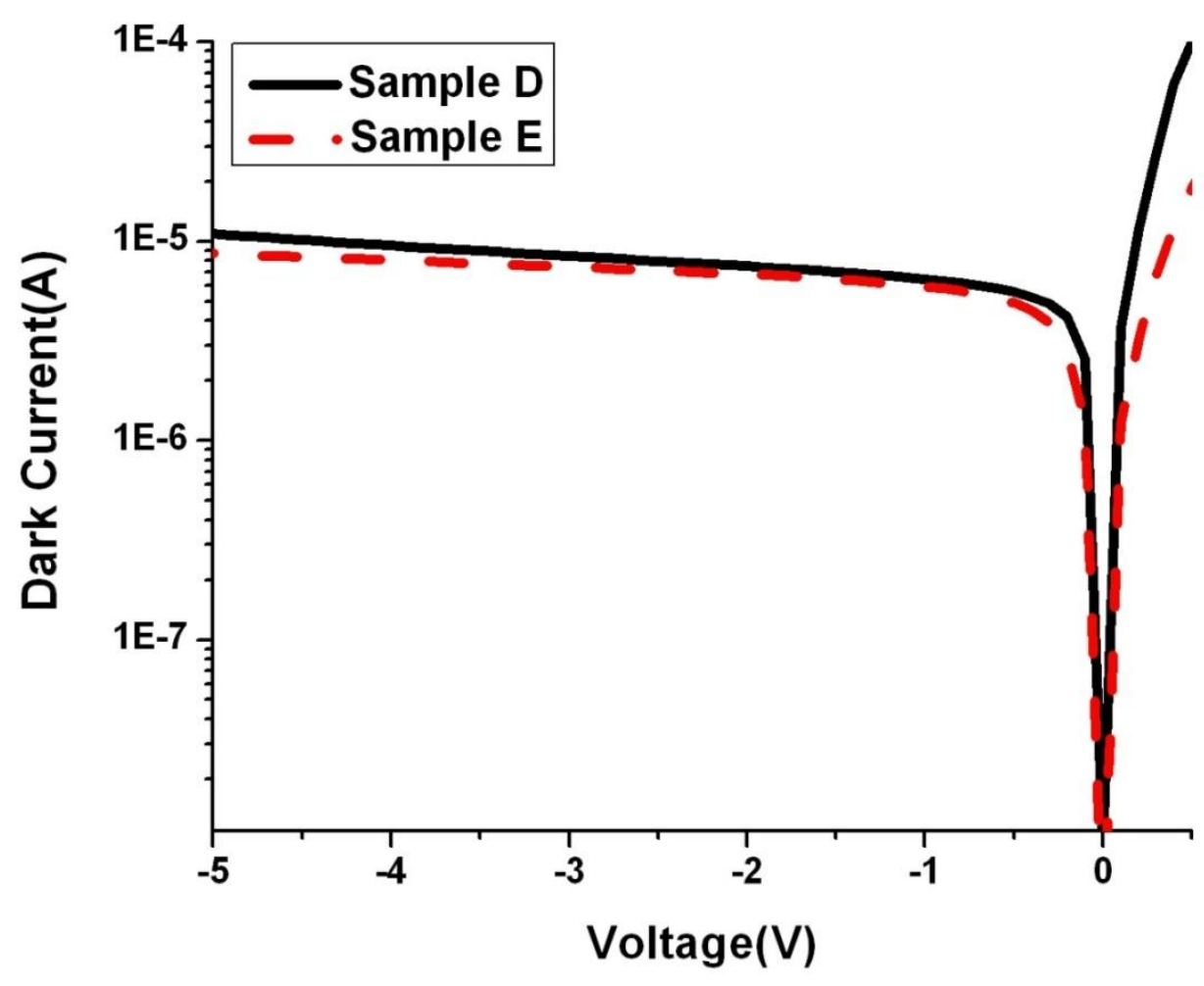

Figure 6.6 Dark current of $350 \mu \mathrm{m}$ devices for each structure at room temperature

Two devices with diameter of $350 \mu \mathrm{m}$ were wire bonded on the same TO-header. The dark current of both devices at room temperature are shown in Figure 6.6. The normal incident photo response for both devices was measured at temperatures between $200 \mathrm{~K}$ and 290K, as shown in Figure 6.7. It was observed that both devices have very similar responsivity, which indicated the energy height of the barrier layer near n-region does not block the photo generated carrier being collected. Therefore, it can be concluded that the carrier transport mechanism is dominated by thermionic emission. 


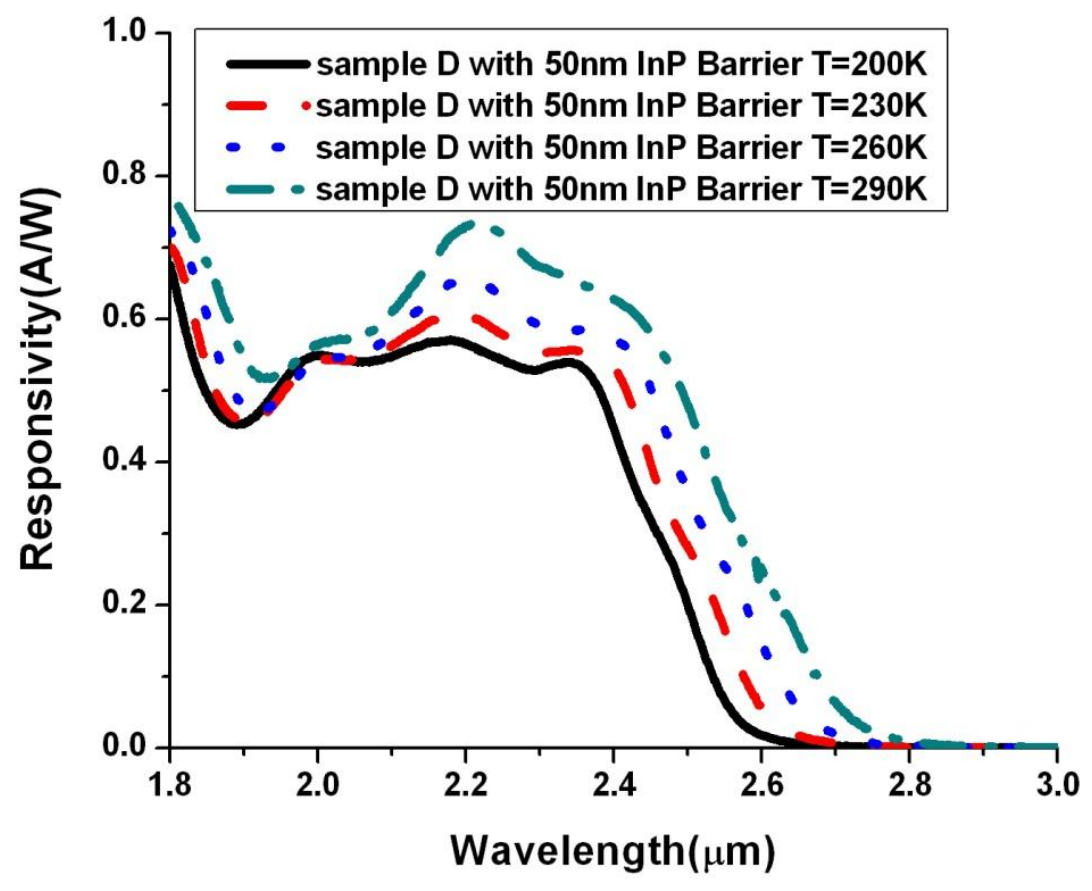

(a)

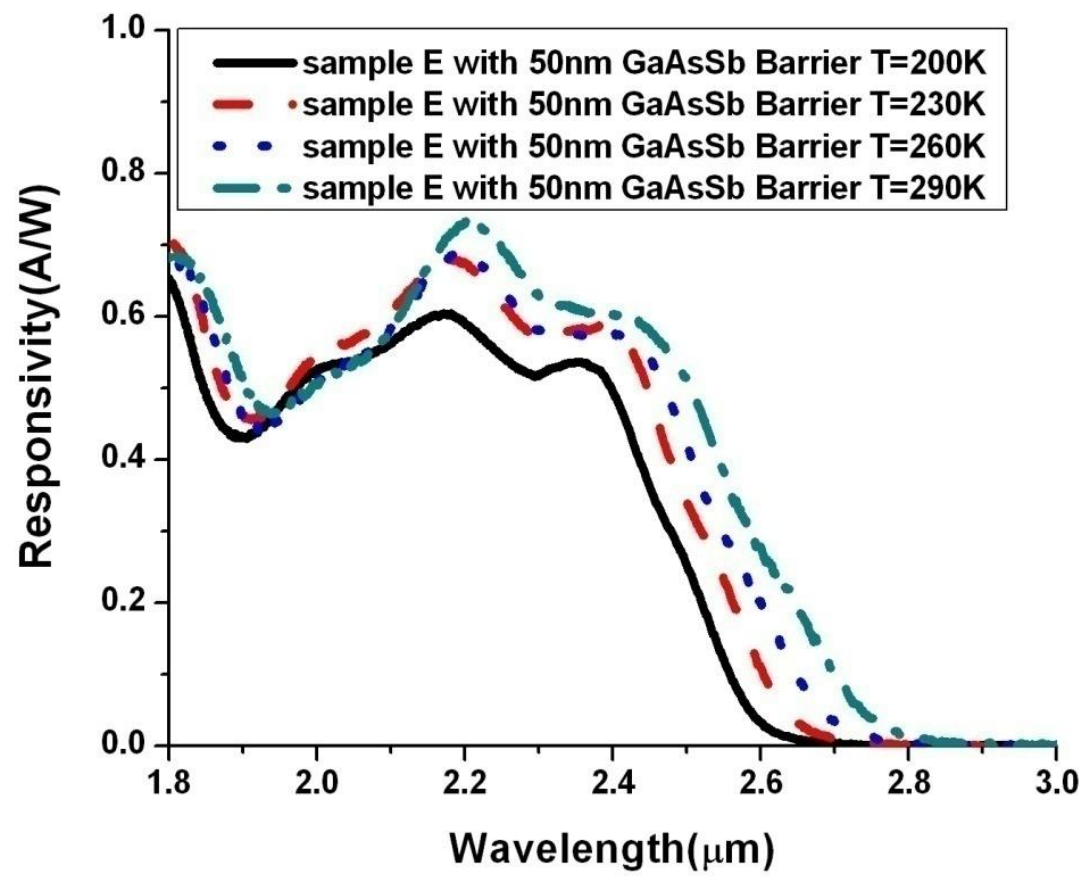

(b) 


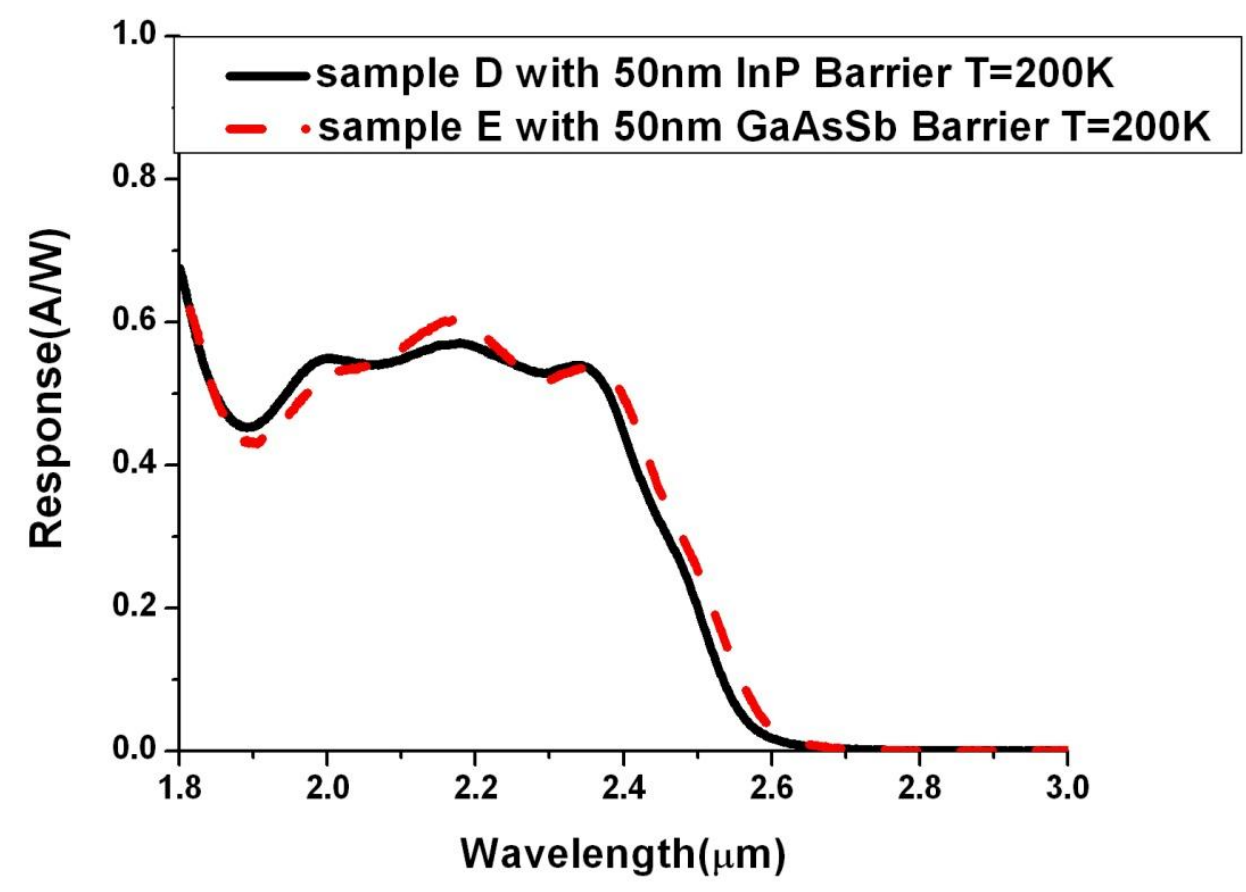

(c)

Figure 6.7 (a) Normal incident photo response of the device at different temperatures, at reverse bias of $-1 \mathrm{~V}$ for Device A. (b) Normal incident photo response of the device at different temperatures, at reverse bias of $-1 \mathrm{~V}$ for Device $\mathrm{B}$. (c) the photo responses of both devices at $200 \mathrm{~K}$ on the same plot

\subsection{Conclusions}

The carrier transport mechanisms in InGaAs/GaAsSb type-II quantum wells were studied by comparing the responsivity beyond 1800nm of two PIN photodiodes with different energy barrier before the n-region. The results show that thermionic emission is the dominant carrier transport mechanism in photodiodes using InGaAs/GaAsSb quantum well absorption regions. Knowing that, more complicated structures using these type-II quantum wells can be designed to achieve better performance. 


\section{Chapter 7: Conclusions and Future Work}

\subsection{Conclusions}

The objective of this dissertation is to exploit the advantage of the InP based InGaAs/GaAsSb type-II quantum wells photodiodes in order to achieve high performance and high operating temperature photodiodes for short-wave infrared (SWIR) and midwave infrared (MWIR) detection. The performance of these photodiodes indicates that they are very promising alternatives to other infrared photodiodes. The mature processing and epi-layer growth technologies on InP substrates can lead to high uniformity and high performance for near room temperature operation. In the dissertation, I have discussed the design and performance of different $\mathrm{InP}$ based InGaAs/GaAsSb type-II quantum wells photodiodes. The first InP based photodiode using interband absorption was demonstrated with optical response beyond $3 \mu \mathrm{m}$ at room temperature without lattice mismatch issue.

Due to the complication of the type-II quantum wells structure design, the six-band $\mathrm{k} \cdot \mathrm{p}$ method was described and discussed in terms of detection wavelength and wave function overlap optimization. Based on the simulation, it is found that in order to maximize the transition wavelength and wave function overlap under strain compensated condition, the thickness of the InGaAs layer should be larger than that of the GaAsSb layer, and the GaAsSb layer should be compressively strained, while InGaAs layer should be tensile strained. Furthermore, the additional compressive strain in the quantum wells can help extend the detection wavelength or reduce the $\mathrm{Sb}$ composition in GaAsSb layers. 
The comparison of the PIN photodiodes for the SWIR/MWIR spectral regions using strain compensated InGaAs/GaAsSb QWs and lattice matched InGaAs/GaAsSb QWs was presented in this dissertation. The research has shown that the device with 100 pairs of $7 \mathrm{~nm} \mathrm{In}_{0.34} \mathrm{Ga}_{0.66} \mathrm{As} / 5 \mathrm{~nm} \mathrm{GaAs}_{0.25} \mathrm{Sb}_{0.75}$ strain compensated type-II quantum wells absorption region has an optical response out to $3.2 \mu \mathrm{m}$, while the device with 100 pairs of $7 \mathrm{~nm} \mathrm{In}_{0.53} \mathrm{Ga}_{0.47} \mathrm{As} / 5 \mathrm{~nm} \mathrm{GaAs}{ }_{0.5} \mathrm{Sb}_{0.5}$ lattice matched type-II QWs absorption region has an optical response out to $2.7 \mu \mathrm{m}$. The strain compensated devices show detectivity of $1.4 \times 10^{9} \mathrm{~cm} \cdot \mathrm{Hz}^{1 / 2} \cdot \mathrm{W}^{-1}$ at $\lambda=2.7 \mu \mathrm{m}$ at $290 \mathrm{~K}$ and $1.5 \times 10^{10} \mathrm{~cm} \cdot \mathrm{Hz}^{1 / 2} \cdot \mathrm{W}^{-1}$ at $200 \mathrm{~K}$. For $\lambda=3.0 \mu \mathrm{m}$, the detectivity $\mathrm{D}^{*}$ is $2.0 \times 10^{8} \mathrm{~cm} \cdot \mathrm{Hz}^{1 / 2} \cdot \mathrm{W}^{-1}$ at $290 \mathrm{~K}$ and increases to $1.0 \times 10^{9}$ $\mathrm{cm} \cdot \mathrm{Hz}^{1 / 2} \cdot \mathrm{W}^{-1}$ at $200 \mathrm{~K}$. They are the first results at $3 \mu \mathrm{m}$ demonstrated on InP substrate using interband absorption but without using lattice-mismatched layers. Furthermore, the new strain compensated InGaAs/GaAsSb QWs PIN photodiodes was studied, which has the similar detection wavelength compared to conventional strain compensated Sample B but with lower Sb composition in the GaAsSb layer. The detectivity performances of all these devices along with Sidhu's initial device [34] are plotted in Figure 7.1 for summary.

Moreover, the carrier transport mechanism in the type-II quantum wells was studied in order to improve the carrier collection efficiency, and results show that the thermionic emission is the dominant way for the photo generated electrons to get out of the quantum wells. 


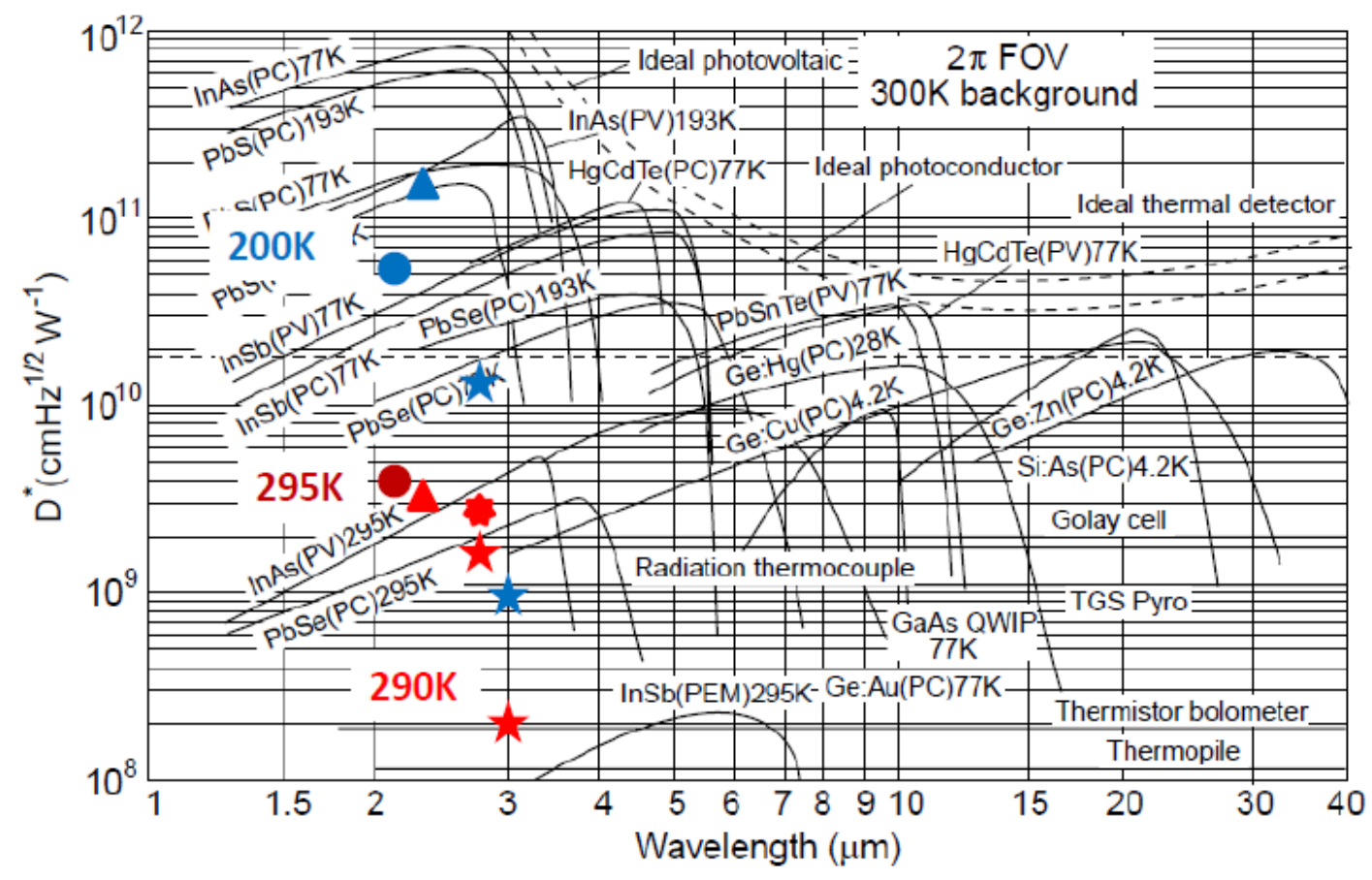

Figure 7.1 Comparison between the detectivities of Sidhu's device (dot), Sample A (triangle), Sample B (5-point star) and Sample C (7-point star) with that of various other infrared detectors

\subsection{Future Work}

The InP based type-II photodiodes discussed in the dissertation shows very encouraging results. Moreover, there is still room for improvement in device performance in terms of improving the detectivity performance and extending the detection wavelength. Some of the future works are recommended as below:

\subsubsection{Optimize the Structure of New Strain Compensated Sample}

The new strain compensated Sample $\mathrm{C}$ has been demonstrated to achieve similar detection wavelength compared to Sample B, but with lower Sb composition in the GaAsSb. However, the advantages of this new strain compensated structure have not been fully explored yet, since the potential to detect long wavelength beyond $4 \mu \mathrm{m}$ has not 
been realized. Further work of growing strained InGaAs and GaAsSb layers with high crystal quality and required composition are necessary. Moreover, the selection of the tensile barrier layer can be further optimized in order to minimize the carrier recapture probability, thus improving the carrier collection efficiency at low reverse bias. Using a tensile barrier such as AlAsSb or GaInP with similar conduction band alignment with compressive strained GaAsSb layer may reduce the recapture probability.

\subsubsection{InGaAs(N)/GaAsSb Type-II Quantum Wells for MWIR Detection}

The quaternary dilute nitride material $\mathrm{InGaAsN}$ is one of the candidates to extend the operation wavelength. It has been demonstrated for $1.3 \mu \mathrm{m}$ emitting diodes lasers on GaAs substrate [119] and InGaAsN-GaAsSb type-II quantum wells on GaAs substrate have also been realized with emission wavelength beyond $1.5 \mu \mathrm{m}[120,121]$. It has been found that replacing a small amount $(<5 \%)$ of the As with nitrogen in the InGaAs compound reduced the energy gap and modified the electronic structure due to its large bowing factor $[122,123]$. The type-II quantum well structure with the dilute-N InGaAsN on InP substrate can also further extend the detection wavelength based on the band anticrossing (BAC) model [122] and the six-band k.p method calculations as shown in Figure 7.2. 


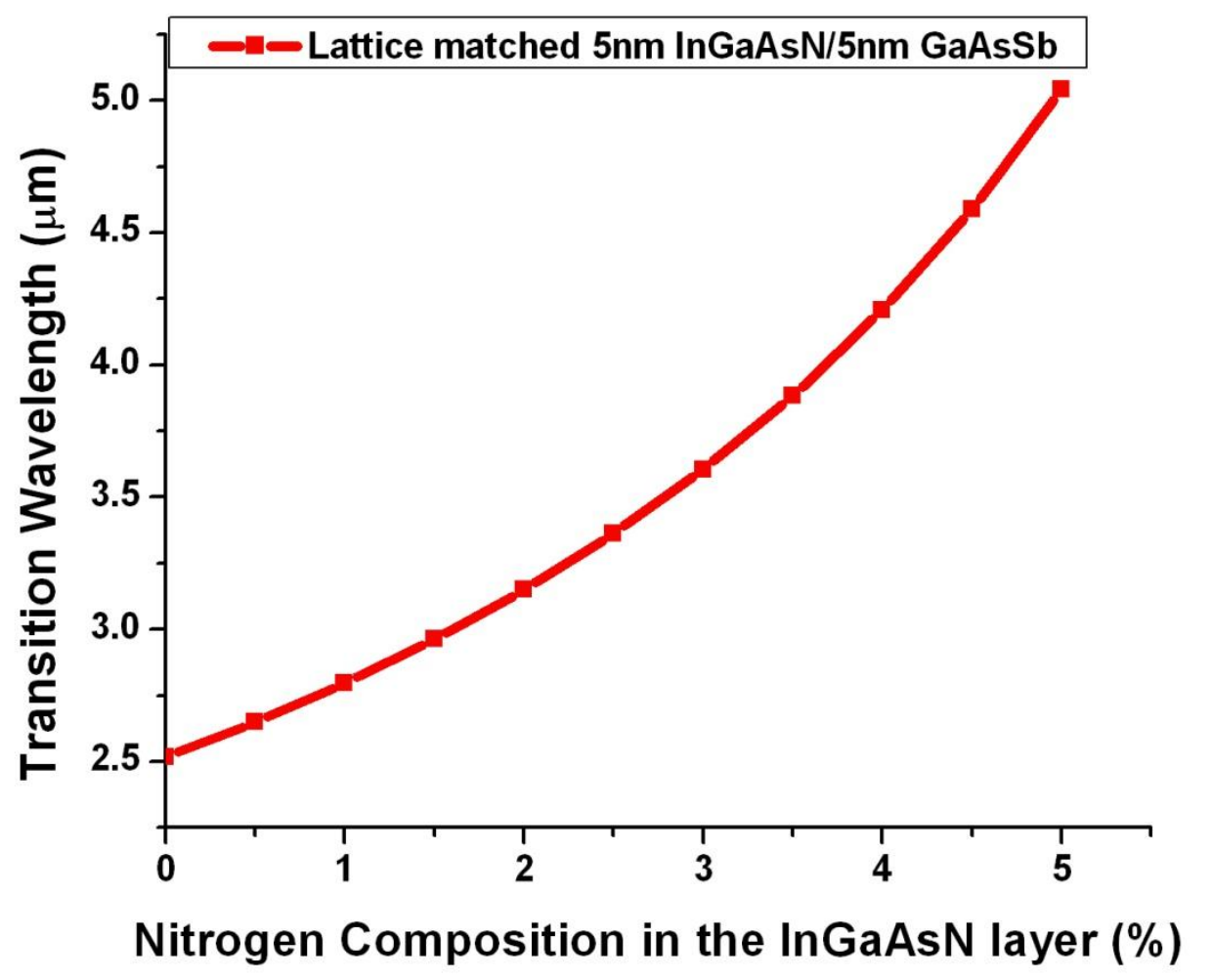

Figure 7.2 Transition wavelength as a function of N\% in the InGaAsN layer

Figure 7.2 shows how the transition wavelength increases as the nitrogen concentration changes in the lattice matched $5 \mathrm{~nm} \mathrm{In}_{0.53} \mathrm{Ga}_{0.47} \mathrm{As}_{1-\mathrm{x}} \mathrm{N}_{\mathrm{x}} / 5 \mathrm{~nm} \mathrm{GaAs} \mathrm{Ga}_{0.5} \mathrm{Sb}_{0.5}$ quantum wells. It is possible to cover the whole MWIR range by incorporating $\mathrm{N}$ into the InGaAsN layer based on Figure 7.2. However, growing high quality, high $\mathrm{N}$ concentration InGaAsN layer on InP substrate is also challenging [124, 125].

\subsubsection{Resonant Cavity Enhanced (RCE) Photodiodes}

The total number of the type-II quantum wells can be increased to enhance the quantum efficiency of the photodiodes. However, a higher operation voltage may be required in order to fully deplete the absorption region, if the background doping 
concentration is not minimized. Furthermore, the dark current would increase and carrier collection efficiency would decrease if the thickness of the absorption region is increased. The trade-offs are undesirable in terms of optimize the detectivity of the photodiodes.

Resonant cavity enhanced structure provides an alternative way to improve the quantum efficiency. In resonant cavity enhanced structures, the absorption region is sandwiched between two mirrors. High quantum efficiency can be achieved with thin absorption region. The main challenge of this structure is meeting the requirements for the mirrors needed for absorption enhancement. The mainstay mirror technology for RCE photodiodes is the Distributed Bragg Reflector (DBR). These mirrors use the index of refraction difference between two layers to achieve the needed reflection. Since the index of refraction difference between the materials lattice-matched to InP substrate is small, very thick mirrors are needed for MWIR operation, which is not a valuable option [126]. Therefore, different approaches to design the high performance mirror for the MWIR range should be further investigated.

\subsubsection{Heterogeneous Integration of Type-II p-i-n Photodiodes on a Silicon-on- Insulator Waveguide Circuit}

Integration of the discrete optoelectronic components on a waveguide platform would open opportunities for a whole new range of applications for mid-wave infrared systems. Silicon photonics has many advantages including low cost and direct compatibility with CMOS processing. It has been demonstrated to be compatible for fabrication with photonics integrated circuits in the telecommunication wavelength range [127]. Given its transparency from 1.2 to $3.5 \mu \mathrm{m}$, it is possible to realize integrated the SWIR/MWIR spectroscopic systems on the platform by combining active optoelectronic devices on 
silicon on insulator passive waveguide circuits. Since the InP based devices have been bonded to SOI wafer using wafer bond technology [127], it should also be possible to bond the InP based type-II quantum wells photodiodes on the SOI waveguide circuit similar to the schematic shown in Figure 7.3.

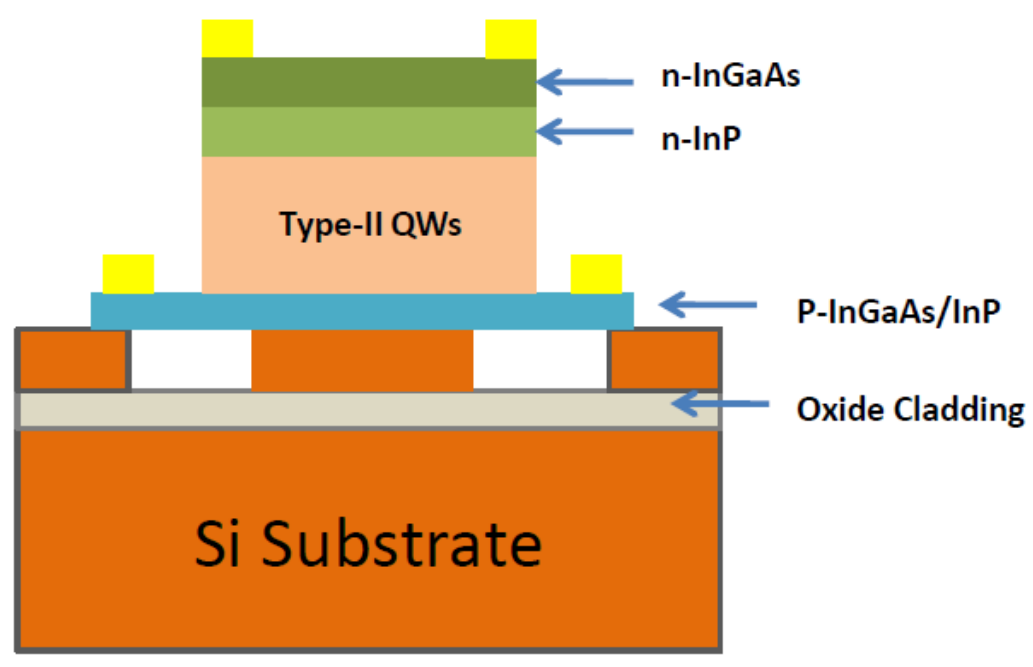

Figure 7.3 Cross-section schematic of the device 


\section{Appendix A: Process Flows}

Photodiodes with top-illuminated mesa can be fabricated from the wafer using the standard process flow as below.

\begin{tabular}{|c|c|c|c|c|}
\hline No. & Step & Mask & Description & Comment \\
\hline 1. & $\begin{array}{l}\text { Cut and mark } \\
\text { wafers }\end{array}$ & & Cut wafers, scratch and mark on rear side of wafer & \\
\hline 2. & Clean & & $\begin{array}{ll}\text { Rinse consecutively in } \\
- & \text { Acetone } \\
- & \text { Isopropanol (IPA) } \\
- & \text { DI water (DI) } \\
- & \text { Blow dry with nitrogen }\end{array}$ & \\
\hline 3. & $\mathrm{PECVD} \mathrm{SiO}_{2}$ & & Deposit $\sim 2000 \AA \mathrm{SiO}_{2}$ & $\begin{array}{l}\text { PECVD, SiO2p35t.prc } \\
\text { 20min }\end{array}$ \\
\hline 4. & Clean & & See 2 & \\
\hline 5. & Bake-out & & Bake-out solvent on hotplate: $130^{\circ} \mathrm{C}, 5 \mathrm{~min}$ & \\
\hline 6. & Spin PR & & $\begin{array}{l}\text { Spin promoter HMDS } \\
\text { Spin photo resist AZ5214 with 4000rpm for } 40 \mathrm{sec} \\
\text { Pre-bake: } 90^{\circ} \mathrm{C}, 3 \mathrm{~min}\end{array}$ & \\
\hline 7. & $\begin{array}{l}\text { Clean mask } \\
\text { and bake out } \\
\text { solvents }\end{array}$ & & $\begin{array}{l}\text { Put mask in PR stripper, rinse DI } \\
\text { Blow dry with nitrogen, bake out solvents } 100^{\circ} \mathrm{C} \text { in oven }\end{array}$ & \\
\hline 8. & $\begin{array}{l}\text { Align and } \\
\text { Expose }\end{array}$ & Mesa & Align and expose 55 secs, soft contact, on MBJ4 aligner & \\
\hline 9. & Develop & & Develop $30 \mathrm{sec}$ in AZ300K, put in DI, blow nitrogen & \\
\hline 10. & Post-bake & & Post-bake hotplate: $110^{\circ} \mathrm{C}, 3 \mathrm{~min}$ & \\
\hline 11. & Etch $\mathrm{SiO}_{2}$ & & Etch 2 min in BOE (10 : 1), DI, blow nitrogen & \\
\hline 12. & Remove PR & & Rinse: Acetone, IPA, DI, then nitrogen blow dry & \\
\hline 13. & Wet etch & & $\begin{array}{l}\text { InP wet etch: } \mathrm{HCl}: \mathrm{H}_{3} \mathrm{PO}_{4}=1: 3 \text { around } 35 \mathrm{~s} \text {, to remove the } \\
\text { p-InP region }\end{array}$ & \\
\hline
\end{tabular}




\begin{tabular}{|c|c|c|c|c|}
\hline & & & $\begin{array}{l}\mathrm{H}_{3} \mathrm{PO}_{4}: \mathrm{H}_{2} \mathrm{O}_{2}: \mathrm{H}_{2} \mathrm{O}=1: 1: 10 \text { for } 2 \mathrm{mins} \text { to etch } \mathrm{i}- \\
\text { InGaAs/GaAsSb region and stop at } n-\mathrm{InP} \text {, } \\
\mathrm{HCl}: \mathrm{H}_{3} \mathrm{PO}_{4}=1: 3 \text { around } 10 \text { s, to etch the n-InP region }\end{array}$ & \\
\hline 14. & Remove $\mathrm{SiO}_{2}$ & & BOE 2mins & \\
\hline 15. & $\begin{array}{l}\mathrm{SiO}_{2} \\
\text { passivation }\end{array}$ & & $\begin{array}{l}\text { Deposit } \sim 2000 \AA \mathrm{SiO}_{2} \text {, the appropriate thickness of } \mathrm{SiO}_{2} \\
\text { can be used as AR coating for the photodiodes. }\end{array}$ & $\begin{array}{l}\text { PECVD, SiO2p35t.prc } \\
\text { 20min }\end{array}$ \\
\hline 16. & Clean & & See 2 & \\
\hline 17. & Bake-out & & Bake-out solvent on hotplate: $130^{\circ} \mathrm{C}, 5 \mathrm{~min}$ & \\
\hline 18. & Spin PR & & $\begin{array}{l}\text { Spin promoter HMDS } \\
\text { Spin photo resist AZ5214 with 4000rpm for } 40 \mathrm{sec} \\
\text { Pre-bake: } 90^{\circ} \mathrm{C}, 3 \mathrm{~min}\end{array}$ & \\
\hline 19. & $\begin{array}{l}\text { Align and } \\
\text { Expose }\end{array}$ & $\begin{array}{l}\text { PN } \\
\text { metal }\end{array}$ & Align and expose $55 \mathrm{sec}$, soft contact, on MBJ4 aligner & \\
\hline 20. & Develop & & Develop $30 \mathrm{sec}$ in AZ300K, put in DI, blow nitrogen & \\
\hline 21. & Etch $\mathrm{SiO}_{2}$ & & Etch 2 min in BOE (10: 1), DI, blow nitrogen & \\
\hline 22. & $\begin{array}{l}\text { p-n metal } \\
\text { deposition }\end{array}$ & & Deposit: $400 \AA \mathrm{Ti}$, then $1000 \AA \mathrm{Au}$ & e-beam evaporator \\
\hline 23. & Lift off & & Remove PR: put wafer in Acetone, & $\begin{array}{l}\text { use shortly ultrasonic } \\
\text { bath }\end{array}$ \\
\hline 24. & Rinse & & Rinse in Acetone, IPA, DI, then $\mathrm{N}_{2}$ blow dry & Rinse \\
\hline
\end{tabular}

In the process flow above, positive photo resist AZ 5214 is used for photolithography.

The recipe for negative photo resist NLOF 2020 is shown below for convenience.

\section{1) No HMDS \\ 2) Spin NLOF $20205.5 \mathrm{k} \mathrm{rpm}$ for 30s}

3) Soft bake at $110^{\circ} \mathrm{C}$ for $1 \mathrm{~min}$ 
4) Expose $0.5 \mathrm{~s}$ on MBJ4 aligner

5) After exposure bake at $110^{\circ} \mathrm{C}$ for $1 \mathrm{mins}$ (DO NOT FORGET THIS STEP)

6) Develop AZ $300 \mathrm{MIF}$ approx 30s check before

7) Post Bake $100{ }^{\circ} \mathrm{C}$ for $90 \mathrm{~s}$

Moreover, in the standard process flow, the photodiodes was passivated with $\mathrm{SiO} 2$, as supplementary, passivation with $1000 \mathrm{~nm}$ SU-8 is also discussed. Generally speaking, when SU8 is used to passivate the photodiodes, metal contacts can be processed first, and some simple tests such as I-V measurement can be performed on these devices. Then SU8 is spun on the whole wafer to passivate the photodiodes. In order to open a window for metal contact, SU-8 developer is used to develop the SU-8 on the top of the metal. The recipe for SU-8 passivation is shown below.

1) Spin HMDS twice

2) Bake for $10 \mathrm{~min}$ at $90{ }^{\circ} \mathrm{C}$

3) Spin coat $\mathrm{SU}-8$ at $2000 \mathrm{rpm}$ for $40 \mathrm{~s}$

4) Bake for $90 \mathrm{~s}$ at $90^{\circ} \mathrm{C}$

5) Expose 30s on MBJ4 aligner

6) Bake $90 \mathrm{~s}$ at $90{ }^{\circ} \mathrm{C}$, then wait $2 \mathrm{~min}$

7) Develop in SU-8 developer

8) IPA rinse

9) Post-bake $130{ }^{\circ} \mathrm{C}$ for 2 minutes then $150 / 160{ }^{\circ} \mathrm{C}$ for 15 minutes

In addition, thicker gold (such as 500nm) may be used for easier wire bonding, but the gold may be hard to get lift off in Acetone. The following recipe using LOR is shown below for reference.

1) Spin LOR3000rpm $30 \mathrm{sec}$ 
2) Bake $5 \min$ on $160^{\circ} \mathrm{C}$

3) Spin your second layer of PR(such AZ5214)

4) Bake for 2 min (depends on what second PR you use)

5) Develop in AZ400K (1:3) for roughly 1 min (closely monitor the development to make sure there is the right amount of undercut)

6) Metal deposition with e-beam evaporator

7) Lift off in Acetone first with ultrasonic bath, then rinse the LOR in NMP(NMethyl-2-pyrrolidone)

8) IPA rinse 


\section{Appendix B: Publications}

[1] Baile Chen, W.Y. Jiang, Jinrong Yuan, A. L. Holmes Jr, Bora. M. Onat. "Demonstration of a Room Temperature InP-based Photodetector Operating beyond $3 \mu \mathrm{m}$ ", IEEE PHOTONICS TECHNOLOGY LETTERS, VOL. 23, NO.

4, FEBRUARY 15, 2011, 218-220

[2] Baile Chen, W.Y. Jiang, Jinrong Yuan, A. L. Holmes Jr, Bora. M. Onat "SWIR/MWIR InP-based PIN Photodiodes with InGaAs/GaAsSb Type-II Quantum Wells" IEEE QUANTUM ELECTRONICS, VOL. 47, ISSUE 9, September, 2011, 1244-1250

[3] Baile Chen, W.Y. Jiang, A. L. Holmes Jr, "Design of Strain Compensated InGaAs/GaAsSb Type-II Quantum Well Structures for Mid-infrared Photodiodes" Optical and Quantum Electronics, Volume 44, Issue 3 (2012), Page 103-109

[4] Baile Chen, Qiugui Zhou, Dion C.McIntosh, Jinrong Yuan, Yaojia Chen, Wenlu Sun, Joe C. Campbell, A. L. Holmes Jr. "Natural Lithography Nano-sphere Texturing as Antireflective Layer on InGaAs PIN Photodiodes" Electronics Letters, October 11, 2012, Volume 48, Issue 21, p.1340-1341

[5] Baile Chen, A. L. Holmes Jr, "Optical Gain Modeling of InP Based InGaAs(N)/GaAsSb Type-II Quantum Wells Laser for Mid-Infrared Emission" Optical and Quantum Electronics: Volume 45, Issue 2 (2013), Page 127-134

[6] Baile Chen, Jinrong Yuan, A. L. Holmes Jr. "Dark Current Modeling of InP based SWIR and MWIR InGaAs/GaAsSb Type-II MQW Photodiodes”, Optical and Quantum Electronics: Volume 45, Issue 3 (2013), Page 271-277 
[7] Jinrong Yuan, Baile Chen, Archie L. Holmes, Jr. "Near-infrared quantum efficiency of uncooled photodetectors based on InGaAs/GaAsSb quantum wells lattice-matched to InP" Electronics Letters, September 29, 2011 Volume: 47 Issue:20, page(s): $1144-1145$

[8] Wenjie Chen, Baile Chen, Jinrong Yuan, Archie Holmes, Patrick Fay "Bulk and interfacial deep levels observed in In0.53Ga0.47As/GaAs0.5Sb0.5 multiple quantum well photodiode” Applied Physics Letters 101, 052107 (2012)

[9] Jinrong Yuan, Baile Chen, Archie L. Holmes, Jr. "Improved Quantum Efficiency of InGaAs/InP Photodetectors using a Ti/Au-SiO2 Phase-Matched-Layer reflector", Electronics Letters Volume 48, Issue 19, p.1230-1232

[10] Baile Chen, Jinrong Yuan, A. L. Holmes Jr. "Modeling of the electrical characteristics of SWIR/MWIR InGaAs/GaAsSb type-II MQW photodiodes" SPIE Defense, Security, and Sensing 2011, 25 - 29, April 2011, Orlando, Florida, USA

[11] Baile Chen, A. L. Holmes Jr, W.Y. Jiang, Jinrong Yuan, "Design of Strain Compensated InGaAs/GaAsSb Type-II Quantum Well Structures for Midinfrared Photodiodes" 11th Annual NUSOD Conference, 5-8 September 2011, Rome, Italy, Paper ThC4

[12] Baile Chen, Wenlu Sun, Joe C. Campbell and A. L. Holmes Jr. "Quantum Efficiency Modeling of PIN Photodiodes with InGaAs/GaAsSb Quantum Wells Absorption Region” 2011 IEEE Photonics Conference, 9-13 October 2011, Arlington, Virginia, USA, Page 35, Paper MD4 
Onat. "Modeling of the type-II InGaAs/GaAsSb quantum well designs for midinfrared laser diodes by k·p method" SPIE Defense, Security, and Sensing 2012, 23 - 27 April 2012, Baltimore, Maryland, USA

[14] Wenjie. Chen, Baile Chen, J. Yuan, A. Holmes, and P. Fay, "Characterization and Impact of Traps in Lattice-Matched and StrainCompensated In1-xGaxAs/GaAs1-ySby Multiple Quantum Well Photodiodes" Device Research Conference Jun 18-20, 2012 Penn State University, University Park, PA, USA.

[15] Weiyang Jiang, Baile Chen, Jinrong Yuan, A. L. Holmes Jr. "Design and characterization of strain-compensated GaInAs/GaAsSb type-II MQW structure with operation wavelength at $\sim 3 \mu \mathrm{m}$ " SPIE Defense, Security, and Sensing 2010, 5 - 9, April 2010, Orlando, Florida, USA.

[16] Wenlu Sun, Xiaoguang Zheng, Zhiwen Lu, Baile Chen, Archie L. Holmes Jr. and Joe C. Campbell "Numerical Simulation of InAlAs/InAlGaAs Tandem Avalanche Photodiodes” 2011 IEEE Photonics Conference, 9-13 October 2011, Arlington, Virginia, USA, Page 280, Paper TuK3. 


\section{References}

[1] $E M$

spectrum.

Available:

http://www.stumbleupon.com/su/2kaMdH/:_j9x8zim:4wfA.enE/www.colourthera pyhealing.com/colour/images/electromagnetic-spectrum.jpg/

[2] W. Herschel, "Experiments on the refrangibility of the invisible rays of the Sun," Philosophical Transactions of the Royal Society of London (1776-1886), vol. 90, pp. 284-29, 1800.

[3] G. P. Agrawal, "Fiber-Optic Communication Systems," 4th ed: Wiley, 2010.

[4] D. K. Mynbaev and L. L. Scheiner, "Fiber-Optic Communications Technology," 1st ed: Prentice Hall, 2000.

[5] S. D. Gunapala, S. V. Bandara, A. Singh, J. K. Liu, S. B. Rafol, E. M. Luong, et al., "640 x 486 long-wavelength two-color GaAs/AlGaAs quantum well infrared photodetector (QWIP) focal plane array camera," Ieee Transactions on Electron Devices, vol. 47, pp. 963-971, May 2000.

[6] S. D. Gunapala, S. V. Bandara, J. K. Liu, W. Hong, M. Sundaram, P. D. Maker, et al., "Long-wavelength 640 x 486 GaAs/AlGaAs quantum well infrared photodetector snap-shot camera," Ieee Transactions on Electron Devices, vol. 45, pp. 1890-1895, Sep 1998.

[7] J. T. Olesberg, M. A. Arnold, C. Mermelstein, J. Schmitz, and J. Wagner, "Tunable laser diode system for noninvasive blood glucose measurements," Applied Spectroscopy, vol. 59, pp. 1480-4, Dec 2005. 
[8] H. I. Schiff, G. I. Mackay, and S. D. Nadler, "Some applications of NIR tunable diodes for remote sensing," Infrared Physics \& Technology, vol. 37, pp. 39-43, Feb 1996.

[9] C. Fischer, E. Sorokin, I. T. Sorokina, and M. W. Sigrist, "Photoacoustic monitoring of gases using a novel laser source tunable around $2.5 \mathrm{mu} \mathrm{m}$," Optics and Lasers in Engineering, vol. 43, pp. 573-582, Mar-May 2005.

[10] D. C. Klonoff, "Noninvasive blood glucose monitoring," Diabetes Care, vol. 20, pp. 433-437, Mar 1997.

[11] K. V. Larin, M. S. Eledrisi, M. Motamedi, and R. O. Esenaliev, "Noninvasive blood glucose monitoring with optical coherence tomography - A pilot study in human subjects," Diabetes Care, vol. 25, pp. 2263-7, Dec 2002.

[12] S. Kayashima, T. Arai, M. Kikuchi, N. Sato, N. Nagata, O. Takatani, et al., "New noninvasive transcutaneous approach to blood glucose monitoring: successful glucose monitoring on human 75 g OGTT with novel sampling chamber," Ieee Transactions on Biomedical Engineering, vol. 38, pp. 752-7, Aug 1991.

[13] K. Maruo, T. Oota, M. Tsurugi, T. Nakagawa, H. Arimoto, M. Tamura, et al., "New methodology to obtain a calibration model for noninvasive near-infrared blood glucose monitoring," Applied Spectroscopy, vol. 60, pp. 441-449, Apr 2006.

[14] K. Michel, B. Bureau, C. Boussard-Pledel, T. Jouan, J. L. Adam, K. Staubmann, et al., "Monitoring of pollutant in waste water by infrared spectroscopy using chalcogenide glass optical fibers," Sensors and Actuators B-Chemical, vol. 101, pp. 252-259, Jun 152004. 
[15] F. Charpentier, B. Bureau, J. Troles, C. Boussard-Pledel, K. Michel-Le Pierres, F. Smektala, et al., "Infrared monitoring of underground $\mathrm{CO} 2$ storage using chalcogenide glass fibers," Optical Materials, vol. 31, pp. 496-500, Jan 2009.

[16] P. Lucas, M. A. Solis, D. Le Coq, C. Juncker, M. R. Riley, J. Collier, et al., "Infrared biosensors using hydrophobic chalcogenide fibers sensitized with live cells," Sensors and Actuators B-Chemical, vol. 119, pp. 355-362, Dec 72006.

[17] J. B. Ko, O. K. Cho, Y. O. Kim, and K. Yasuda, "Body metabolism provides a foundation for noninvasive blood glucose monitoring," Diabetes Care, vol. 27, pp. 1211-1212, May 2004.

[18] L. Sandstrom, S. Backstrom, H. Ahlberg, S. Hojer, and A. G. Larsson, "Gas monitoring using semiconductor lasers operating in the $2 \mathrm{mu} \mathrm{m}$ wavelength region," Infrared Physics \& Technology, vol. 39, pp. 69-75, Mar 1998.

[19] The Global Climate System. Available: http://www.nature.com/scitable/knowledge/library/the-global-climate-system$\underline{74649049}$

[20] X. M. Zhu and J. M. Kahn, "Free-space optical communication through atmospheric turbulence channels," Ieee Transactions on Communications, vol. 50, pp. 1293-1300, Aug 2002.

[21] W. Lei and C. Jagadish, "Lasers and photodetectors for mid-infrared 2-3 mu m applications," Journal of Applied Physics, vol. 104, Nov 2008.

[22] F.-Y. Yue, J. Shao, Y.-F. Wei, X. Lu, H. Wei, J.-R. Yang, et al., "Temperaturedependent absorption spectra investigation of shallow levels in $\mathrm{HgCdTe}$ grown by liquid phase epitaxy," Acta Physica Sinica, vol. 56, pp. 2878-2881, May 2007. 
[23] P. Tribolet, J. P. Chatard, P. Costa, and A. Manissadjian, "Progress in HgCdTe homojunction infrared detectors," Journal of Crystal Growth, vol. 184, Feb 1998.

[24] Y. Chang, C. Fulk, J. Zhao, C. H. Grein, and S. Sivananthan, "Molecular beam epitaxy growth of $\mathrm{HgCdTe}$ for high performance infrared photon detectors," Infrared Physics \& Technology, vol. 50, pp. 284-290, Apr 2007.

[25] A. Rogalski, "HgCdTe infrared detector material: history, status and outlook," Reports on Progress in Physics, vol. 68, pp. 2267-2336, Oct 2005.

[26] J. Shao, X. Lue, W. Lu, F. Yue, W. Huang, N. Li, et al., "Cutoff wavelength of Hg1-xCdxTe epilayers by infrared photoreflectance spectroscopy," Applied Physics Letters, vol. 90, p. 171101, Apr 232007.

[27] J. D. Phillips, K. Moazzami, J. Kim, D. D. Edwall, D. L. Lee, and J. M. Arias, "Uniformity of optical absorption in $\mathrm{HgCdTe}$ epilayer measured by infrared spectromicroscopy," Applied Physics Letters, vol. 83, p. 3701, Nov 32003.

[28] B. F. Levine, "QUANTUM-WELL INFRARED PHOTODETECTORS," Journal of Applied Physics, vol. 74, pp. R1-R81, Oct 1993.

[29] A. V. Barve and S. Krishna, "Quantum Dot Infrared Photodetectors," in Advances in Infrared Photodetectors. vol. 84, S. D. Gunapala, D. R. Rhiger, and C. Jagadish, Eds., ed, 2011, pp. 153-193.

[30] J. C. Campbell and A. Madhukar, "Quantum-dot infrared photodetectors," Proceedings of the Ieee, vol. 95, pp. 1815-1827, Sep 2007.

[31] P. Martyniuk and A. Rogalski, "Quantum-dot infrared photodetectors: Status and outlook," Progress in Quantum Electronics, vol. 32, pp. 89-120, 2008. 
[32] F. Fuchs, U. Weimer, W. Pletschen, J. Schmitz, E. Ahlswede, M. Walther, et al., "High performance InAs/Ga1-xInxSb superlattice infrared photodiodes," Applied Physics Letters, vol. 71, pp. 3251-3253, Dec 1997.

[33] D. L. Smith and C. Mailhiot, "PROPOSAL FOR STRAINED TYPE-II SUPERLATTICE INFRARED DETECTORS," Journal of Applied Physics, vol. 62, pp. 2545-2548, Sep 1987.

[34] R. Sidhu, N. Duan, J. C. Campbell, and A. L. Holmes, "A long-wavelength photodiode on InP using lattice-matched GaInAs-GaAsSb type-II quantum wells," Ieee Photonics Technology Letters, vol. 17, pp. 2715-2717, Dec 2005.

[35] R. K. Pathria, Statistical Mechanics, 2nd ed.: Butterworth-Heinemann, 1996.

[36] A. Rogalski, "Infrared detectors: status and trends," Progress in Quantum Electronics, vol. 27, pp. 59-210, 2003.

[37] A. Rogalski, "New material systems for third generation infrared photodetectors," Opto-Electronics Review, vol. 16, p. 25, Dec 2008.

[38] A. Rogalski, J. Antoszewski, and L. Faraone, "Third-generation infrared photodetector arrays," Journal of Applied Physics, vol. 105, p. 091101, May 2009.

[39] A. Rogalski, "History of infrared detectors," Opto-Electronics Review, vol. 20, pp. 279-308, Sep 2012.

[40] bandgap vs. the lattice constant. Available: http://www.tf.unikiel.de/matwis/amat/semi_en/kap_5/backbone/r5_1_4.html

[41] P. Norton, "HgCdTe infrared detectors," Opto-Electronics Review, vol. 10, pp. 48-70, Sep 2002. 
[42] J. Singh, Electronic and Optoelectronic Properties of Semiconductor Structures, 1st ed.: Cambridge University Press, 2003.

[43] S. D. Gunapala and S. V. Bandara, "Quantum well infrared photodetector (QWIP) focal plane arrays," Intersubband Transitions in Quantum Wells: Physics and Device Applications I, vol. 62, pp. 197-282, 2000.

[44] M. Tadic and Z. Ikonic, "BOUND-FREE INTERSUBBAND ABSORPTION IN P-TYPE DOPED SEMICONDUCTOR QUANTUM-WELLS," Physical Review B, vol. 52, pp. 8266-8275, Sep 1995.

[45] D. K. Sengupta, S. L. Jackson, D. Ahmari, H. C. Kuo, J. I. Malin, S. Thomas, et al., "p-type InGaAs/InP quantum well infrared photodetector with peak response at 4.55 mu m," Applied Physics Letters, vol. 69, pp. 3209-3211, Nov 1996.

[46] S. A. Stoklitsky, P. O. Holtz, B. Monemar, Q. X. Zhao, and T. Lundstrom, "STRAIN EFFECTS ON THE INTERVALENCE-SUBBAND NORMALINCIDENCE ABSORPTION IN A P-TYPE INGAAS/INP QUANTUM-WELL," Applied Physics Letters, vol. 65, pp. 1706-1708, Sep 1994.

[47] F. M. Guo, D. Y. Xiong, W. E. Zhang, and Z. Q. Zhu, "Analysis and comparison of n-AlxGa1-xAs/GaAs QWIPs with different device structures and optical coupling," Infrared Physics \& Technology, vol. 52, pp. 276-280, Nov 2009.

[48] H. T. Jiang and J. Singh, "Radiative and non-radiative inter-subband transition in self assembled quantum dots," Physica E, vol. 2, pp. 720-724, Jul 1998.

[49] E. G. Camargo, K. Ueno, T. Morishita, H. Goto, N. Kuze, K. Sawada, et al., "Performance Improvement of Molecular Beam Epitaxy Grown InSb Photodiodes 
for Room Temperature Operation," Japanese Journal of Applied Physics, vol. 47, pp. 8430-8433, Nov 2008.

[50] R. M. Lin, S. F. Tang, S. C. Lee, C. H. Kuan, G. S. Chen, T. P. Sun, et al., "Room temperature unpassivated InAs p-i-n photodetectors grown by molecular beam epitaxy," Ieee Transactions on Electron Devices, vol. 44, pp. 209\&\#x9;- 213, Feb 1997.

[51] G. A. Sai-Halasz, R. Tsu, and L. Esaki, "A new semiconductor superlattice," Applied Physics Letters, vol. 30, p. 651, 1977.

[52] Available: http://spie.org/x57586.xml?pf=true\&ArticleID=x5..

[53] I. Vurgaftman, J. R. Meyer, and L. R. Ram-Mohan, "Band parameters for III-V compound semiconductors and their alloys," Journal of Applied Physics, vol. 89, pp. 5815-5875, Jun 2001.

[54] A. M. Hoang, G. Chen, A. Haddadi, S. A. Pour, and M. Razeghi, "Demonstration of shortwavelength infrared photodiodes based on type-II InAs/GaSb/AlSb superlattices," Applied Physics Letters, vol. 100, p. 211101, May 2012.

[55] J. Huang, W. Ma, Y. Wei, Y. Zhang, K. Cui, Y. Cao, et al., "How to Use Type II InAs/GaSb Superlattice Structure to Reach Detection Wavelength of 2-3 mu m," Ieee Journal of Quantum Electronics, vol. 48, pp. 1322\&\#x9;- 1326, Oct 2012.

[56] C. Cervera, J. B. Rodriguez, R. Chaghi, H. Ait-Kaci, and P. Christol, "Characterization of midwave infrared InAs/GaSb superlattice photodiode," Journal of Applied Physics, vol. 106, p. 024501, Jul 2009.

[57] A. Khoshakhlagh, E. Plis, S. Myers, Y. D. Sharma, L. R. Dawson, and S. Krishna, "Optimization of InAs/GaSb type-II superlattice interfaces for long-wave (similar 
to $8 \mathrm{mu}$ m) infrared detection," Journal of Crystal Growth, vol. 311, pp. 19011904, Mar 2009.

[58] A. Khoshakhlagh, S. Myers, H. Kim, E. Plis, N. Gautam, S. J. Lee, et al., "LongWave InAs/GaSb Superlattice Detectors Based on nBn and Pin Designs," Ieee Journal of Quantum Electronics, vol. 46, pp. 959-964, Jun 2010.

[59] G. Ariyawansa, M. Grupen, J. M. Duran, J. E. Scheihing, T. R. Nelson, and M. T. Eismann, "Design and modeling of InAs/GaSb type II superlattice based dualband infrared detectors," Journal of Applied Physics, vol. 111, p. 073107, Apr 1 2012.

[60] E. Plis, S. Annamalai, K. T. Posani, S. Krishna, R. A. Rupani, and S. Ghosh, "Midwave infrared type-II InAs/GaSb superlattice detectors with mixed interfaces," Journal of Applied Physics, vol. 100, p. 014510, Jul 2006.

[61] S. A. Pour, E. K. Huang, G. Chen, A. Haddadi, B. M. Nguyen, and M. Razeghi, "High operating temperature midwave infrared photodiodes and focal plane arrays based on type-II InAs/GaSb superlattices," Applied Physics Letters, vol. 98, p. 143501, Apr 2011.

[62] B. M. Onat, X. Jiang, and M. Itzler, "A systematic approach to dark current reduction in InGaAs-based photodiode arrays for shortwave infrared imaging," in LEOS Annual Meeting Conference Proceedings, 2009, pp. 231-232.

[63] M. R. Gokhale, J. Wei, H. S. Wang, and S. R. Forrest, "Growth and characterization of small band gap (similar to $0.6 \mathrm{eV}$ ) InGaAsN layers on InP," Applied Physics Letters, vol. 74, pp. 1287-1289, Mar 1999. 
[64] M. Yoshikawa, K. Miura, Y. Iguchi, and Y. Kawamura, "Optical characterization of InGaAsN layers grown on InP substrates," Journal of Crystal Growth, vol. 311, pp. 1745-1747, Mar 2009.

[65] J. Wei, M. R. Gokhale, K. J. Thomson, and S. R. Forrest, "InGaAsPN-InP-based photodetectors for long wavelength (lambda > $1.65 \mathrm{mu}$ m) applications," Ieee Photonics Technology Letters, vol. 12, pp. 68-70, Jan 2000.

[66] K. Kohler, J. Wagner, P. Ganser, D. Serries, T. Geppert, M. Maier, et al., "The realization of long-wavelength (lambda $<=2.3 \mathrm{mu}$ m) Ga1-xInxAs1-xNy quantum wells on InP by molecular-beam epitaxy," Journal of PhysicsCondensed Matter, vol. 16, pp. S2995-S3008, Aug 2004.

[67] K. Miura, Y. Nagai, Y. Iguchi, H. Okada, and Y. Kawamura, "Improvement of crystal quality of thick InGaAsN layers grown on InP substrates by adding antimony," Journal of Crystal Growth, vol. 301, pp. 575-578, Apr 2007.

[68] J. C. Dries, M. R. Gokhale, K. J. Thomson, S. R. Forrest, and R. Hull, "Strain compensated In1-xGaxAs(x < 0.47) quantum well photodiodes for extended wavelength operation," Applied Physics Letters, vol. 73, pp. 2263-2265, Oct 1998.

[69] J. C. Dries, M. R. Gokhale, and S. R. Forrest, "A $2.0 \mathrm{mu}$ m cutoff wavelength separate absorption, charge, and multiplication layer avalanche photodiode using strain-compensated InGaAs quantum wells," Applied Physics Letters, vol. 74, pp. 2581-2583, May 1999.

[70] G. Boehm, M. Grau, O. Dier, K. Windhorn, E. Roenneberg, J. Rosskopf, et al., "Growth of InAs-containing quantum wells for InP-based VCSELs emitting at 2.3 mu m," Journal of Crystal Growth, vol. 301, pp. 941-944, Apr 2007. 
[71] C. Li, Y. Zhang, K. Wang, Y. Gu, H. Li, and Y. Li, "Distinction investigation of InGaAs photodetectors cutoff at $2.9 \mathrm{mu}$ m," Infrared Physics \& Technology, vol. 53, pp. 173-176, May 2010.

[72] Solid State Division. Available: http://www.sales.hamamatsu.com/en/products/solid-state-division/ingaas-pinphotodiodes/long-wavelength-type/part-g8423-03.php

[73] H. Takasaki, Y. Kawamura, T. Katayama, A. Yamamoto, and N. Inoue, "Electroluminescence of $\mathrm{In} 0.53 \mathrm{Ga} 0.47 \mathrm{As} / \mathrm{GaAs} 0.5 \mathrm{Sb} 0.5$ type II multiple quantum well diodes lattice-matched to InP," Journal of Crystal Growth, vol. 227, pp. 294-297, Jul 2001.

[74] A. Yamamoto, Y. Kawamura, H. Naito, and N. Inoue, "Optical properties of GaAs0.5Sb0.5 and In0.53Ga0.47As/GaAs0.5Sb0.5 type II single hetero-structures lattice-matched to InP substrates grown by molecular beam epitaxy," Journal of Crystal Growth, vol. 201, pp. 872-876, May 1999.

[75] Y. Sugiyama, T. Fujii, Y. Nakata, S. Muto, and E. Miyauchi, "CONDUCTIONBAND EDGE DISCONTINUITY OF INGAAS/GAASSB HETEROSTRUCTURES LATTICE-MATCHED TO INP GROWN BY MOLECULAR-BEAM EPITAXY," Journal of Crystal Growth, vol. 95, pp. 363366, Feb 1989.

[76] H. Takasaki, Y. Kawamura, T. Katayama, A. Yamamoto, H. Naito, and N. Inoue, "Photoluminescence properties of $\mathrm{In} 0.53 \mathrm{Ga} 0.47 \mathrm{As} / \mathrm{GaAs} 0.5 \mathrm{Sb} 0.5$ type II quantum well structures lattice-matched to InP," Applied Surface Science, vol. 159, pp. 528-531, Jun 2000. 
[77] J. Y. T. Huang, L. J. Mawst, T. F. Kuech, X. Song, S. E. Babcock, C. S. Kim, et al., "Design and characterization of strained InGaAs/GaAsSb type-II 'W' quantum wells on InP substrates for mid-IR emission," Journal of Physics D-Applied Physics, vol. 42, p. 025108, Jan 212009.

[78] B. Chen, W. Y. Jiang, and A. L. Holmes, Jr., "Design of strain compensated InGaAs/GaAsSb type-II quantum well structures for mid-infrared photodiodes," Optical and Quantum Electronics, vol. 44, pp. 103-109, Jun 2012.

[79] H.Inada, K.Miura, Y.Nagai, M.Tsubokura, A.Moto, Y.Iguchi, et al., "Low dark current SWIR photodiode with InGaAs/GaAsSb Type II quantum wells grown on InP substrate," presented at the Indium Phosphide \& Related Materials, 2009.

[80] R. Sidhu, L. Zhang, N. Tan, N. Duan, J. C. Campbell, A. L. Holmes, et al., "2.4 mu m cutoff wavelength avalanche photodiode on InP substrate," Electronics Letters, vol. 42, pp. 181-182, Feb 22006.

[81] D. S. G. Ong, J. S. Ng, Y. L. Goh, C. H. Tan, S. Zhang, and J. P. R. David, "InAlAs Avalanche Photodiode With Type-II Superlattice Absorber for Detection Beyond 2 mu m," Ieee Transactions on Electron Devices, vol. 58, pp. 486\&\#x9;489, Feb 2011.

[82] Brillouin Zone. Available: http://en.wikipedia.org/wiki/File:Brillouin Zone \%281st, FCC\%29.svg

[83] J. P. Loehr, Physics of Strained Quantum Well Lasers, 1st ed.: Springer, 1997.

[84] C. S. Chang and S. L. Chuang, "MODELING OF STRAINED-QUANTUMWELL LASERS WITH SPIN-ORBIT-COUPLING," Ieee Journal of Selected Topics in Quantum Electronics, vol. 1, pp. 218\&\#x9;- 229, Jun 1995. 
[85] C. Y. P. Chao and S. L. Chuang, "Spin-orbit-coupling effects on the valence-band structure of strained semiconductor quantum wells," Physical Review B, vol. 46, pp. 4110-4122, Aug 151992.

[86] S. L. Chuang, "EFFICIENT BAND-STRUCTURE CALCULATIONS OF STRAINED QUANTUM-WELLS," Physical Review B, vol. 43, pp. 9649-9661, Apr 151991.

[87] G. B. Liu and S. L. Chuang, "Modeling of Sb-based type-II quantum cascade lasers," Physical Review B, vol. 65, p. 165220, Apr 152002.

[88] T. B. Bahder, "Eight-band k•p model of strained zinc-blende crystals," Phys. Rev. $B$, vol. 41, pp. 11992-12001, 1990.

[89] S. Ben Radhia, N. Fraj, I. Saidi, and K. Boujdaria, "The eight-level k center dot p model for the conduction and valence bands of InAs, InP, InSb," Semiconductor Science and Technology, vol. 22, pp. 427-434, Apr 2007.

[90] M. V. Kisin, B. L. Gelmont, and S. Luryi, "Boundary-condition problem in the Kane model," Physical Review B, vol. 58, pp. 4605-4616, Aug 151998.

[91] B. A. Foreman, "Elimination of spurious solutions from eight-band $k$ center dot $p$ theory," Physical Review B, vol. 56, pp. 12748-12751, Nov 151997.

[92] B. A. Foreman, "Choosing a basis that eliminates spurious solutions in $\mathrm{k}$ center dot p theory," Physical Review B, vol. 75, pp. 12748-12751, Jun 2007.

[93] Q. Zhao, T. Mei, and D. H. Zhang, "Elimination of spurious solutions from k.p theory with Fourier transform technique and Burt-Foreman operator ordering," Journal of Applied Physics, vol. 111, p. 053702, Mar 12012. 
[94] R. G. Veprek, S. Steiger, and B. Witzigmann, "Ellipticity and the spurious solution problem of k center dot p envelope equations," Physical Review B, vol. 76, p. 165320 , Oct 2007.

[95] U. Ekenberg, "Nonparabolicity effects in a quantum well: Sublevel shift, parallel mass, and Landau levels," Physical Review B, vol. 40, pp. 7714-7726, Oct 15 1989.

[96] M. Sugawara, N. Okazaki, T. Fujii, and S. Yamazaki, "CONDUCTION-BAND AND VALENCE-BAND STRUCTURES IN STRAINED IN1-XGAXAS/INP QUANTUM-WELLS ON (001) INP SUBSTRATES," Physical Review B, vol. 48, pp. 8102-8118, Sep 151993.

[97] J. Hu, X. G. Xu, J. A. H. Stotz, S. P. Watkins, A. E. Curzon, M. L. W. Thewalt, et al., "Type II photoluminescence and conduction band offsets of GaAsSb/InGaAs and GaAsSb/InP heterostructures grown by metalorganic vapor phase epitaxy," Applied Physics Letters, vol. 73, p. 2799, Nov 81998.

[98] S. Jourba, M. P. Besland, M. Gendry, M. Garrigues, J. L. Leclercq, P. RojoRomeo, et al., "2 mu m resonant cavity enhanced InP/InGaAs single quantum well photo-detector," Electronics Letters, vol. 35, pp. 1272-1274, Jul 1999.

[99] Y. Yonezawa, R. Hiraike, K. Miura, Y. Iguchi, and Y. Kawamura, "Growth and characterization of strain-compensated InGaAs/GaAsSb type II multiple quantum wells on InP substrate," Physica E-Low-Dimensional Systems \& Nanostructures, vol. 42, pp. 2781-2783, Sep 2010.

[100] C. Rohr, P. Abbott, I. Ballard, J. P. Connolly, K. W. J. Barnham, M. Mazzer, et al., "InP-based lattice-matched InGaAsP and strain-compensated InGaAs/InGaAs 
quantum well cells for thermophotovoltaic applications," Journal of Applied Physics, vol. 100, p. 114510, Dec 12006.

[101] N. J. Ekins-Daukes, K. Kawaguchi, and J. Zhang, "Strain-balanced criteria for multiple quantum well structures and its signature in X-ray rocking curves," Crystal Growth \& Design, vol. 2, pp. 287-292, Jul-Aug 2002.

[102] P. Harrison, Quantum Wells, Wires, and Dots, 2nd ed.: Wiley, 2005.

[103] S. L. Chuang, "Physics of Photonic Devices," 2nd ed: Wiley, 2009.

[104] Q. K. Yang, F. Fuchs, J. Schmitz, and W. Pletschen, "Investigation of trapassisted tunneling current in $\mathrm{InAs} /(\mathrm{GaIn}) \mathrm{Sb}$ superlattice long-wavelength photodiodes," Applied Physics Letters, vol. 81, p. 4757, Dec 162002.

[105] P. Martyniuk, J. Wrobel, E. Plis, P. Madejczyk, A. Kowalewski, W. Gawron, et al., "Performance modeling of MWIR InAs/GaSb/B-A10.2Ga0.8Sb type-II superlattice nBn detector," Semiconductor Science and Technology, vol. 27, p. 055002 , May 2012.

[106] J. Nguyen, D. Z. Ting, C. J. Hill, A. Soibel, S. A. Keo, and S. D. Gunapala, "Dark current analysis of InAs/GaSb superlattices at low temperatures," Infrared Physics \& Technology, vol. 52, pp. 317-321, Nov 2009.

[107] S. M. Sze and K. K. Ng, Physics of Semiconductor Devices, 3rd ed.: Wiley, 2006.

[108] Z. Ye, "Design, Fabrication and Characterization of Quantum Dot Infrared Photodetectors," Ph.D, The University of Texas at Austin, 2003.

[109] H. D. Vincent, Fundamentals of Infrared Detector Operation \& Testing: Wiley, 1990. 
[110] W. Chen, B. Chen, J. Yuan, A. Holmes, and P. Fay, "Bulk and interfacial deep levels observed in In0.53Ga0.47As/GaAs0.5Sb0.5 multiple quantum well photodiode," Applied Physics Letters, vol. 101, pp. 052107-1, Jul 302012.

[111] Y. Sun, S. F. Cheng, G. Chen, R. L. Woo, and R. F. Hicks, "The structure of indium phosphide (001) treated with trimethylantimony in a metalorganic vaporphase epitaxy reactor," Journal of Applied Physics, vol. 97, May 152005.

[112] S. Weeke, M. Leyer, M. Pristovsek, F. Brunner, M. Weyers, and W. Richter, "Segregation and desorption of antimony in InP (001) in MOVPE," Journal of Crystal Growth, vol. 298, pp. 159-162, Jan 2007.

[113] J. Y. T. Huang, D. P. Xu, J. H. Park, L. J. Mawst, T. F. Kuech, X. Song, et al., "Characteristics of strained GaAs1-ySby $(0.16<=y<=0.69)$ quantum wells on InP substrates," Journal of Physics D-Applied Physics, vol. 40, Dec 212007.

[114] J. Y. T. Huang, D. P. Xu, X. Song, S. E. Babcock, T. F. Kuech, and L. J. Mawst, "Growth of strained GaAs1-ySby and GaAs1-y-zSbyNz quantum wells on InP substrates," Journal of Crystal Growth, vol. 310, Apr 2008.

[115] B. L. Chen, Q. G. Zhou, D. C. McIntosh, J. R. Yuan, Y. J. Chen, W. L. Sun, et al., "Natural lithography nano-sphere texturing as antireflective layer on InP-based pin photodiodes," Electronics Letters, vol. 48, pp. 1340-1341, Oct 2012.

[116] H. Schneider and K. Vonklitzing, "THERMIONIC EMISSION AND GAUSSIAN TRANSPORT OF HOLES IN A GAAS/ALXGA1-XAS MULTIPLE-QUANTUM-WELL STRUCTURE," Physical Review B, vol. 38, pp. 6160-6165, Sep 151988. 
[117] S. Hojfeldt and J. Mork, "Modeling of carrier dynamics in quantum-well electroabsorption modulators," Ieee Journal of Selected Topics in Quantum Electronics, vol. 8, Nov-Dec 2002.

[118] V. Trinite, E. Ouerghemmi, V. Gueriaux, M. Carras, A. Nedelcu, E. Costard, et al., "Modelling of electronic transport in Quantum Well Infrared Photodetectors," Infrared Physics \& Technology, vol. 54, May 2011.

[119] N. Tansu, N. J. Kirsch, and L. J. Mawst, "Low-threshold-current-density 1300-nm dilute-nitride quantum well lasers," Applied Physics Letters, vol. 81, p. 2523, Sep 302002.

[120] L. J. Mawst, J. Y.-T. Huang, D. P. Xu, J.-Y. Yeh, G. Tsvid, T. E. Kuech, et al., "MOCVD-grown dilute nitride type II quantum wells (Invited paper)," Ieee Journal of Selected Topics in Quantum Electronics, vol. 14, pp. 979-991, Jul-Aug 2008.

[121] J. Y. Yeh, L. J. Mawst, A. A. Khandekar, T. F. Kuech, I. Vurgaftman, J. R. Meyer, et al., "Long wavelength emission of InGaAsN/GaAsSb type II "W" quantum wells," Applied Physics Letters, vol. 88, p. 051115, Jan 302006.

[122] W. Shan, W. Walukiewicz, J. W. Ager, E. E. Haller, J. F. Geisz, D. J. Friedman, et al., "Band anticrossing in GaInNAs alloys," Physical Review Letters, vol. 82, pp. 1221-1224, Feb 81999.

[123] W. Shan, W. Walukiewicz, J. W. Ager, E. E. Haller, J. F. Geisz, D. J. Friedman, et al., "Effect of nitrogen on the band structure of GaInNAs alloys," Journal of Applied Physics, vol. 86, p. 2349, Aug 151999. 
[124] A. Ubukata, J. Dong, K. Matsumoto, and Y. Ishihara, "Growth of Ga0.46In0.54NyAs1-y single quantum wells on $\operatorname{InP}(100)$ substrate by metalorganic chemical vapor deposition," Japanese Journal of Applied Physics Part 1-Regular Papers Short Notes \& Review Papers, vol. 39, pp. 5962-5965, Oct 2000.

[125] R. Kudrawiec, T. Sarmiento, P. Poloczek, J. Misiewicz, and J. S. Harris, "Photoreflectance and photoluminescence study of GaInNAsSb layers lattice matched to InP," Journal of Applied Physics, vol. 107, p. 043523, Feb 2010.

[126] D. Feezell, L. A. Johansson, D. A. Buell, and L. A. Coldren, "Efficient modulation of InP-based 1.3-mu m VCSELs with AsSb-based DBRs," Ieee Photonics Technology Letters, vol. 17, pp. 2253-2255, Nov 2005.

[127] G. Roelkens, L. Liu, D. Liang, R. Jones, A. Fang, B. Koch, et al., "III-V/silicon photonics for on-chip and inter-chip optical interconnects," Laser \& Photonics Reviews, vol. 4, pp. 751-779, Nov 2010. 


\section{Vita}

Baile Chen (陈佰乐) was born in Wenzhou, China on August 12th, 1985, the son of Shengcong Chen (陈圣聪) and Xueyue Zhang (张秀月). He began his undergraduate study in University of Science and Technology of China, Hefei, China in 2003, where he received his Bachelor of Science degree in Physics in 2007. He joined the graduate program in Physics at University of Virginia in 2007. In May 2008, he started research on

photodiodes under Dr. Archie Holmes's supervision. And after receiving his Master of Art in Physics in May 2009, he transferred to Department of Electrical and Computer Engineering at University of Virginia to pursue his PhD degree.

Permanent address: No. 12 Luoting Road, Luofu Village, Oubei Town, Yongjia County, Wenzhou, Zhejiang, P.R. China, 325102

This dissertation was typed by the author. 